\title{
A switchable intramolecular hydrogen bond in 5-nitrosopyrimidines
}

\author{
Eliška Procházková, Lucie Čechová, Zlatko Janeba ${ }^{*}$, Martin Dračínský* \\ Institute of Organic Chemistry and Biochemistry, Academy of Sciences of the Czech Republic \\ Flemingovo nam. 2, 166 10, Prague \\ Email:janeba@uochb.cas.cz,dracinsky@uochb.cas.cz
}

\section{Supporting Information (112 pages)}

Table of Contents

Pages

1) The temperature dependence of ${ }^{1} \mathrm{H}$ NMR spectra of compound $\mathbf{5 e}$

2) The dependence of the percentage of rotamer A on substitution

3) The transition state of compound $\mathbf{5 e}$

4) The picture of the compound $\mathbf{3 e}$ dissolved in four different solvents

5) The calculated electronic transitions of compounds $\mathbf{3 a}-\mathbf{3 h}$

6) The graphical representation of molecular orbitals of the compounds $\mathbf{3 e}$ and $\mathbf{3 h}$

7) Characterization of prepared compounds (NMR, HR-MS, UV/VIS)

8) Computational details 
$140^{\circ} \mathrm{C}$
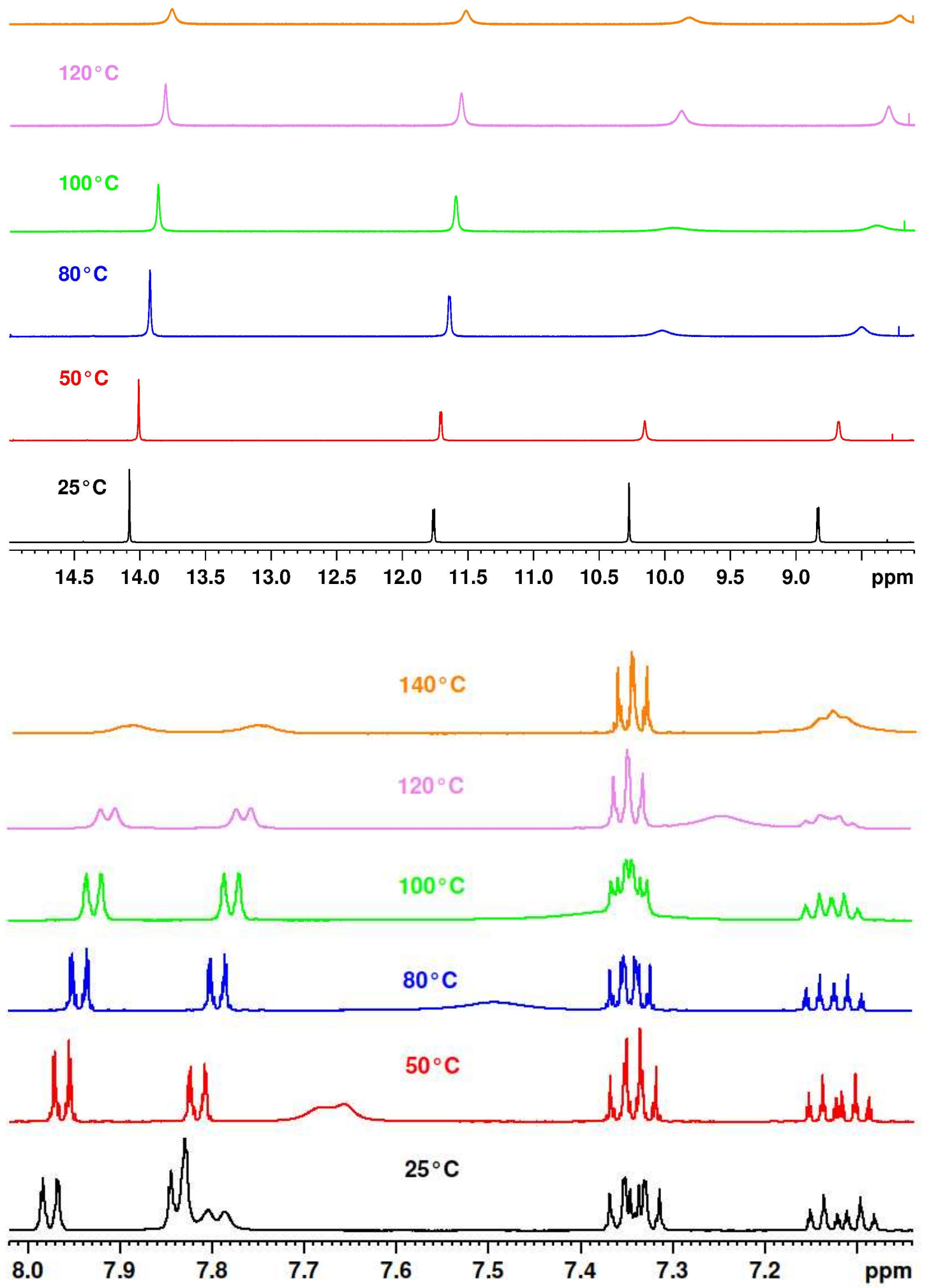

Figure S1. The temperature dependence of ${ }^{1} \mathrm{H}$ NMR spectra of the compound 5e. The NH region (top) and the region of aromatic protons (bottom). 


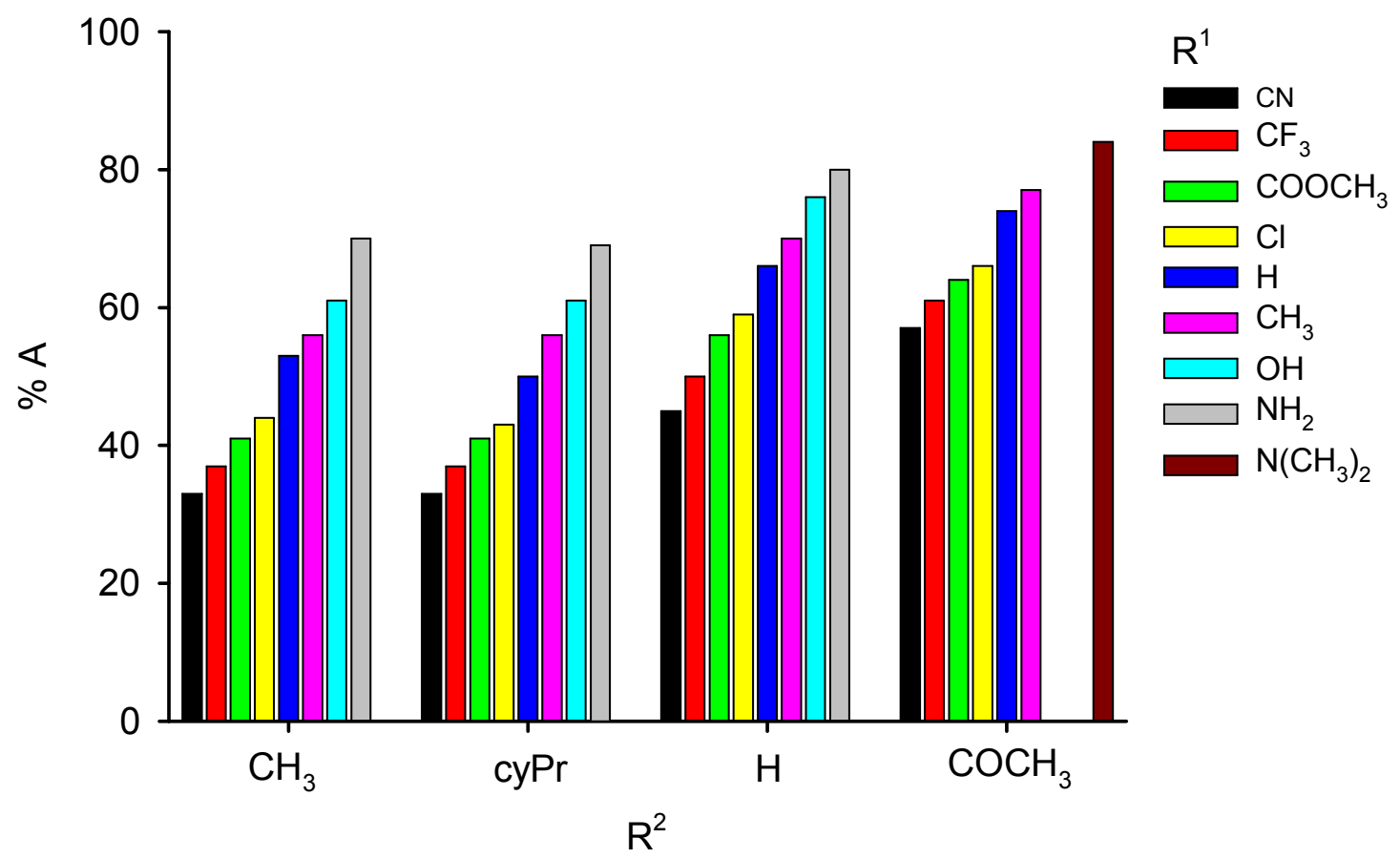

Figure S2. The dependence of the percentage of rotamer A found in DMSO solution of compounds $4-7$ as a function of the nature of the substituents $R^{1}$ and $R^{2}$. 


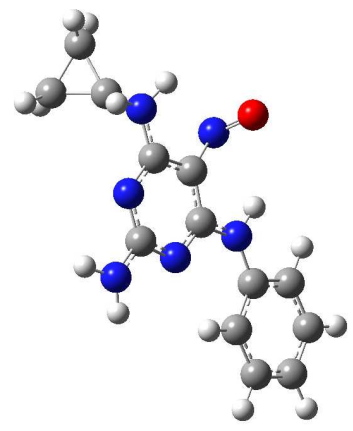

Figure S3. The transition state of compound 5e and the computational details: Cartesian coordinates of optimized structures.

$\begin{array}{cccc}\text { Atomic number } & \mathbf{x} & \mathbf{y} & \mathbf{z} \\ 6 & -0.788028 & -0.806794 & -0.088458 \\ 6 & 0.421320 & -0.081748 & -0.079817 \\ 6 & -1.988343 & -0.072387 & -0.137530 \\ 7 & 0.422059 & 1.255743 & -0.154401 \\ 7 & -1.979908 & 1.266273 & -0.215481 \\ 6 & -0.776896 & 1.856704 & -0.221967 \\ 7 & -3.203528 & -0.707908 & -0.093104 \\ 1 & -3.208681 & -1.697170 & -0.294077 \\ 7 & -0.811604 & -2.237508 & 0.192358 \\ 8 & -0.775778 & -2.945662 & -0.802171 \\ 7 & 1.610417 & -0.769129 & 0.011763 \\ 6 & 2.950374 & -0.337261 & 0.076408 \\ 1 & 1.513320 & -1.773363 & 0.050374 \\ 6 & 3.919590 & -1.345526 & 0.242876 \\ 6 & 5.273394 & -1.028430 & 0.309364 \\ 6 & 5.692721 & 0.302050 & 0.213151 \\ 6 & 3.369558 & 0.999773 & -0.022341 \\ 6 & 4.732249 & 1.301293 & 0.047804 \\ 7 & -0.769251 & 3.219890 & -0.340737 \\ 1 & 3.605774 & -2.384321 & 0.322154 \\ 1 & 6.000365 & -1.825116 & 0.438003 \\ 1 & 6.747712 & 0.552444 & 0.264710 \\ 1 & 2.634157 & 1.778443 & -0.154870 \\ 1 & 5.040499 & 2.340179 & -0.031753 \\ 1 & -1.640449 & 3.701093 & -0.180839 \\ 1 & 0.089717 & 3.702593 & -0.129252 \\ 6 & -4.451816 & -0.014510 & -0.262811 \\ 6 & -5.103527 & 0.628614 & 0.933141 \\ 1 & -5.673012 & -0.646592 & 0.353530 \\ 1 & -4.572412 & 0.491335 & -1.219218 \\ 1 & -4.573382 & 0.559172 & 1.877247 \\ 1 & -5.637856 & 1.560191 & 0.774313 \\ 1 & -6.602600 & -0.601527 & -0.205133 \\ 1 & -5.532730 & -1.561298 & 0.922819\end{array}$




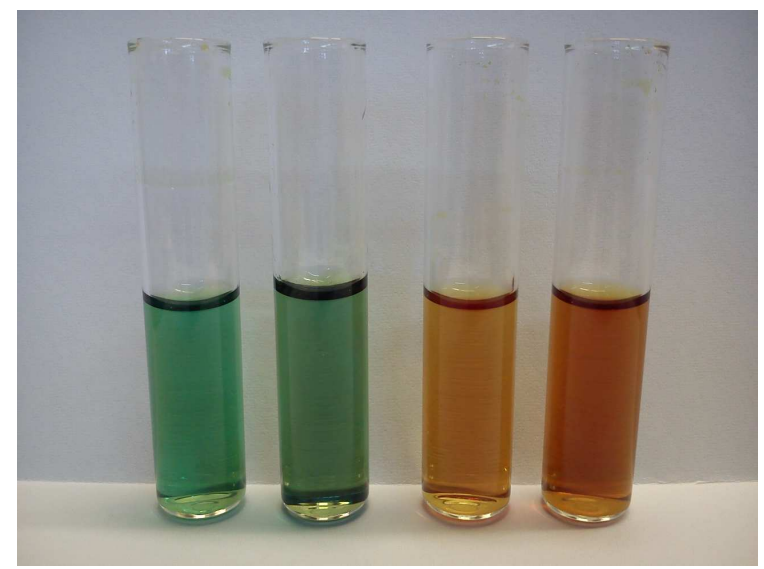

Figure S4. Compound 3e dissolved in different solvents and have different electronic spectra (solvatochromic effect). Solutions of compound $\mathbf{3 e}$ in acetone, DMSO, ethanol and methanol (from left to right).

Table S1. The calculated (B3LYP/6-31+g(d,p)//TD-B3LYP/6-31+g(d,p)/PCM) electronic transitions of compounds $\mathbf{3 a}-\mathbf{3 h}$.

\begin{tabular}{|c|c|c|c|c|c|c|}
\hline Compound & Transition & Orbitals & Orbitals & & $\lambda_{\max }$ & $\begin{array}{l}\text { Oscillator } \\
\text { strength }\end{array}$ \\
\hline \multirow[t]{5}{*}{$\mathbf{3 a}$} & 1 & $70 \rightarrow 71$ & $\mathrm{HOMO} \rightarrow$ LUMO & $\mathrm{n} \rightarrow \pi^{*}$ & 619 & 0.0006 \\
\hline & 2 & $69 \rightarrow 71$ & HOMO-1 $\rightarrow$ LUMO & $\pi \rightarrow \pi^{*}$ & 378 & 0.3805 \\
\hline & 3 & $66 \rightarrow 71$ & HOMO-4 $\rightarrow$ LUMO & $\pi \rightarrow \pi^{*}$ & 317 & 0.0000 \\
\hline & 4 & $68 \rightarrow 71$ & HOMO-2 $\rightarrow$ LUMO & $\pi \rightarrow \pi^{*}$ & 311 & 0.3257 \\
\hline & 5 & $70 \rightarrow 72$ & $\mathrm{HOMO} \rightarrow \mathrm{LUMO}+1$ & $\mathrm{n} \rightarrow \pi^{*}$ & 311 & 0.0000 \\
\hline \multirow[t]{5}{*}{$\mathbf{3 b}$} & 1 & $80 \rightarrow 81$ & $\mathrm{HOMO} \rightarrow \mathrm{LUMO}$ & $\mathrm{n} \rightarrow \pi^{*}$ & 615 & 0.0007 \\
\hline & 2 & $79 \rightarrow 81$ & HOMO-1 $\rightarrow$ LUMO & $\pi \rightarrow \pi^{*}$ & 373 & 0.2821 \\
\hline & 3 & $76 \rightarrow 81$ & HOMO-4 $\rightarrow$ LUMO & $\pi \rightarrow \pi^{*}$ & 315 & 0.0000 \\
\hline & 4 & $78 \rightarrow 81$ & HOMO-2 $\rightarrow$ LUMO & $\pi \rightarrow \pi^{*}$ & 307 & 0.3609 \\
\hline & 5 & $77 \rightarrow 81$ & HOMO-3 $\rightarrow$ LUMO & $\pi \rightarrow \pi^{*}$ & 296 & 0.0006 \\
\hline \multirow[t]{5}{*}{$3 c$} & 1 & $79 \rightarrow 80$ & $\mathrm{HOMO} \rightarrow$ LUMO & $\mathrm{n} \rightarrow \pi^{*}$ & 617 & 0.0006 \\
\hline & 2 & $78 \rightarrow 80$ & HOMO-1 $\rightarrow$ LUMO & $\pi \rightarrow \pi^{*}$ & 383 & 0.3793 \\
\hline & 3 & $75 \rightarrow 80$ & HOMO-4 $\rightarrow$ LUMO & $\pi \rightarrow \pi^{*}$ & 317 & 0.0000 \\
\hline & 4 & $79 \rightarrow 81$ & $\mathrm{HOMO} \rightarrow \mathrm{LUMO}+1$ & $\mathrm{n} \rightarrow \pi^{*}$ & 311 & 0.0000 \\
\hline & 5 & $77 \rightarrow 80$ & HOMO-2 $\rightarrow$ LUMO & $\pi \rightarrow \pi^{*}$ & 311 & 0.3187 \\
\hline \multirow[t]{5}{*}{ 3d } & 1 & $72 \rightarrow 73$ & $\mathrm{HOMO} \rightarrow$ LUMO & $\mathrm{n} \rightarrow \pi^{*}$ & 609 & 0.0007 \\
\hline & 2 & $71 \rightarrow 73$ & HOMO-1 $\rightarrow$ LUMO & $\pi \rightarrow \pi^{*}$ & 396 & 0.2726 \\
\hline & 3 & $68 \rightarrow 73$ & HOMO-4 $\rightarrow$ LUMO & $\pi \rightarrow \pi^{*}$ & 313 & 0.0000 \\
\hline & 4 & $70 \rightarrow 73$ & HOMO-2 $\rightarrow$ LUMO & $\pi \rightarrow \pi^{*}$ & 305 & 0.3631 \\
\hline & 5 & $69 \rightarrow 73$ & HOMO-3 $\rightarrow$ LUMO & $\pi \rightarrow \pi^{*}$ & 296 & 0.0040 \\
\hline \multirow[t]{5}{*}{$3 e$} & 1 & $64 \rightarrow 65$ & $\mathrm{HOMO} \rightarrow$ LUMO & $\mathrm{n} \rightarrow \pi^{*}$ & 608 & 0.0007 \\
\hline & 2 & $63 \rightarrow 65$ & HOMO-1 $\rightarrow$ LUMO & $\pi \rightarrow \pi^{*}$ & 392 & 0.2342 \\
\hline & 3 & $60 \rightarrow 65$ & HOMO-4 $\rightarrow$ LUMO & $\pi \rightarrow \pi^{*}$ & 313 & 0.0000 \\
\hline & 4 & $61 \rightarrow 65$ & HOMO-3 $\rightarrow$ LUMO & $\pi \rightarrow \pi^{*}$ & 313 & 0.0241 \\
\hline & 5 & $62 \rightarrow 65$ & HOMO-2 $\rightarrow$ LUMO & $\pi \rightarrow \pi^{*}$ & 304 & 0.3481 \\
\hline \multirow[t]{5}{*}{$3 f$} & 1 & $67 \rightarrow 69$ & HOMO-1 $\rightarrow$ LUMO & $\mathrm{n} \rightarrow \pi^{*}$ & 606 & 0.0007 \\
\hline & 2 & $68 \rightarrow 69$ & HOMO $\rightarrow$ LUMO & $\pi \rightarrow \pi^{*}$ & 409 & 0.2512 \\
\hline & 3 & $65 \rightarrow 69$ & HOMO-3 $\rightarrow$ LUMO & $\pi \rightarrow \pi^{*}$ & 315 & 0.0149 \\
\hline & 4 & $64 \rightarrow 69$ & HOMO-4 $\rightarrow$ LUMO & $\pi \rightarrow \pi^{*}$ & 313 & 0.0000 \\
\hline & 5 & $66 \rightarrow 69$ & HOMO-2 $\rightarrow$ LUMO & $\pi \rightarrow \pi^{*}$ & 303 & 0.3535 \\
\hline \multirow[t]{2}{*}{$3 g$} & 1 & $67 \rightarrow 69$ & HOMO-1 $\rightarrow$ LUMO & $\mathrm{n} \rightarrow \pi^{*}$ & 602 & 0.0007 \\
\hline & 2 & $68 \rightarrow 69$ & HOMO $\rightarrow$ LUMO & $\pi \rightarrow \pi^{*}$ & 431 & 0.2382 \\
\hline
\end{tabular}




\begin{tabular}{cllclll}
\hline & 3 & $64 \rightarrow 69$ & HOMO-4 $\rightarrow$ LUMO & $\pi \rightarrow \pi^{*}$ & 311 & 0.0000 \\
4 & $66 \rightarrow 69$ & HOMO-2 $\rightarrow$ LUMO & $\pi \rightarrow \pi^{*}$ & 304 & 0.2202 \\
& 5 & $65 \rightarrow 69$ & HOMO-3 $\rightarrow$ LUMO & $\pi \rightarrow \pi^{*}$ & 302 & 0.1405 \\
\hline $3 h$ & 1 & $67 \rightarrow 69$ & HOMO-1 $\rightarrow$ LUMO & $\mathrm{n} \rightarrow \pi *$ & 598 & 0.0007 \\
& 2 & $68 \rightarrow 69$ & HOMO $\rightarrow$ LUMO & $\pi \rightarrow \pi *$ & 483 & 0.2567 \\
& 3 & $63 \rightarrow 69$ & HOMO-5 $\rightarrow$ LUMO & $\pi \rightarrow \pi *$ & 310 & 0.0010 \\
& 4 & $65 \rightarrow 69$ & HOMO-3 $\rightarrow$ LUMO & $\pi \rightarrow \pi *$ & 309 & 0.0085 \\
& 5 & $66 \rightarrow 69$ & HOMO-2 $\rightarrow$ LUMO & $\pi \rightarrow \pi *$ & 304 & 0.3234 \\
\hline
\end{tabular}

Table S2. Graphical representation of molecular orbitals of compounds $\mathbf{3 e}$ and $\mathbf{3 h}$.

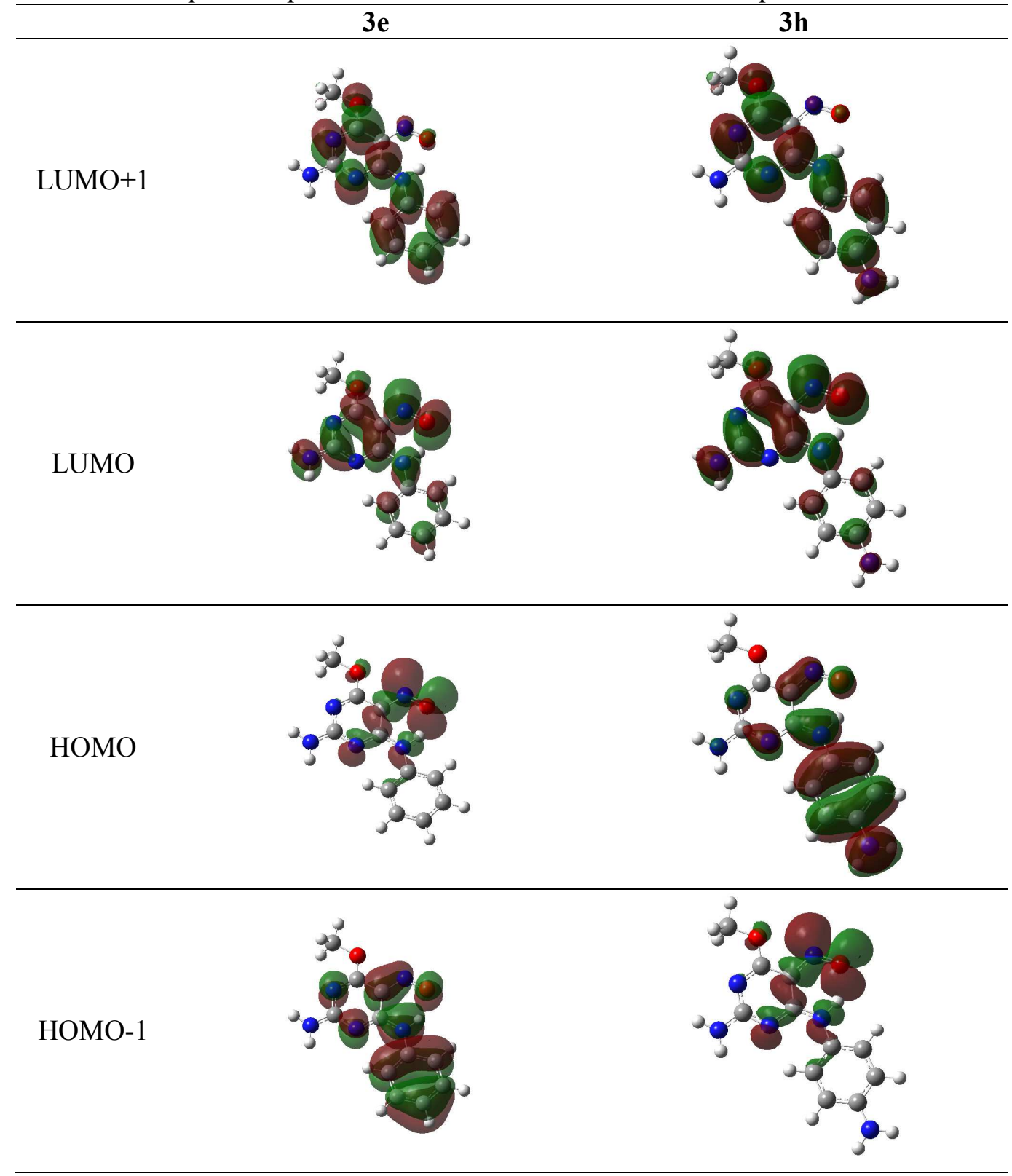




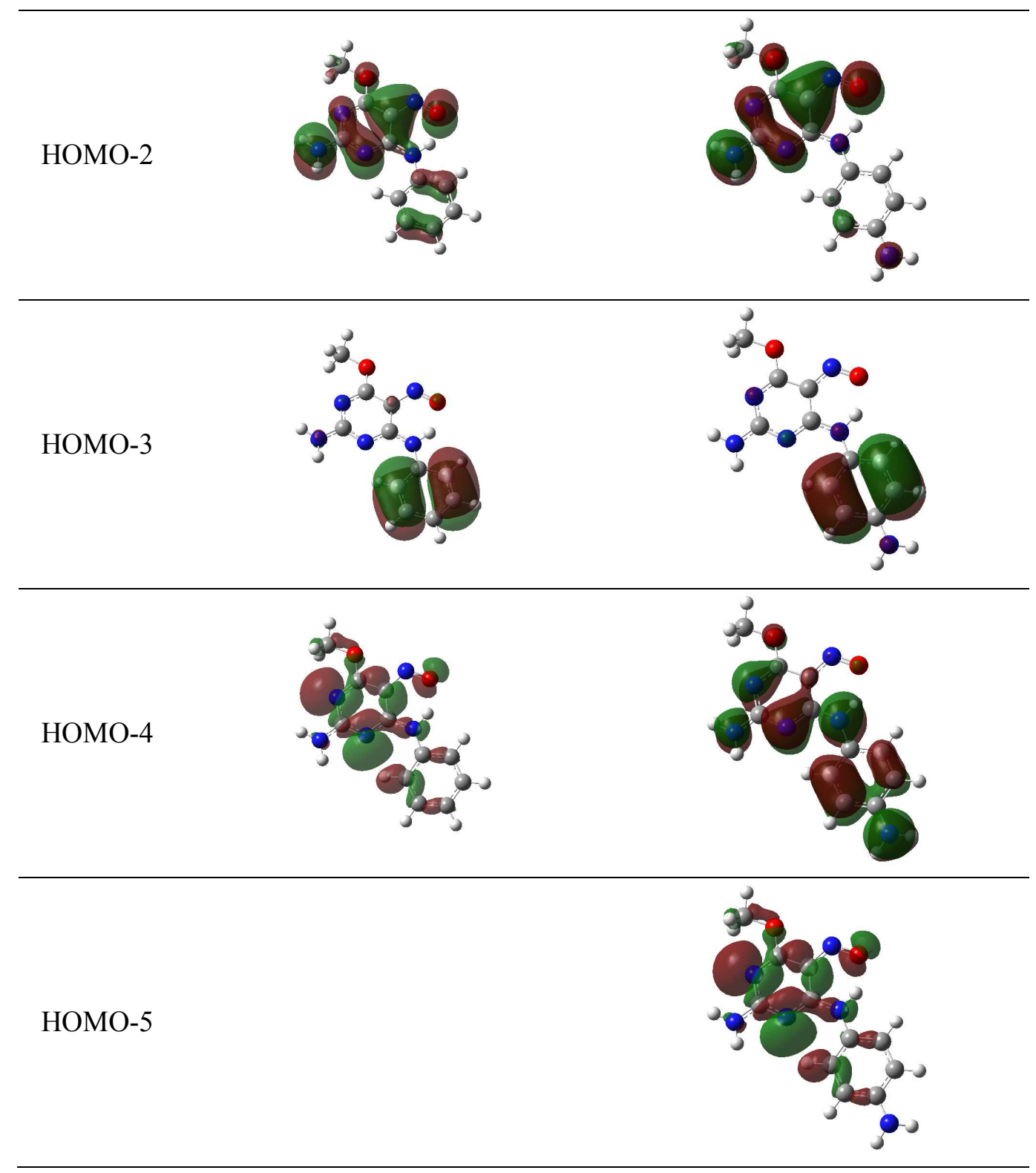




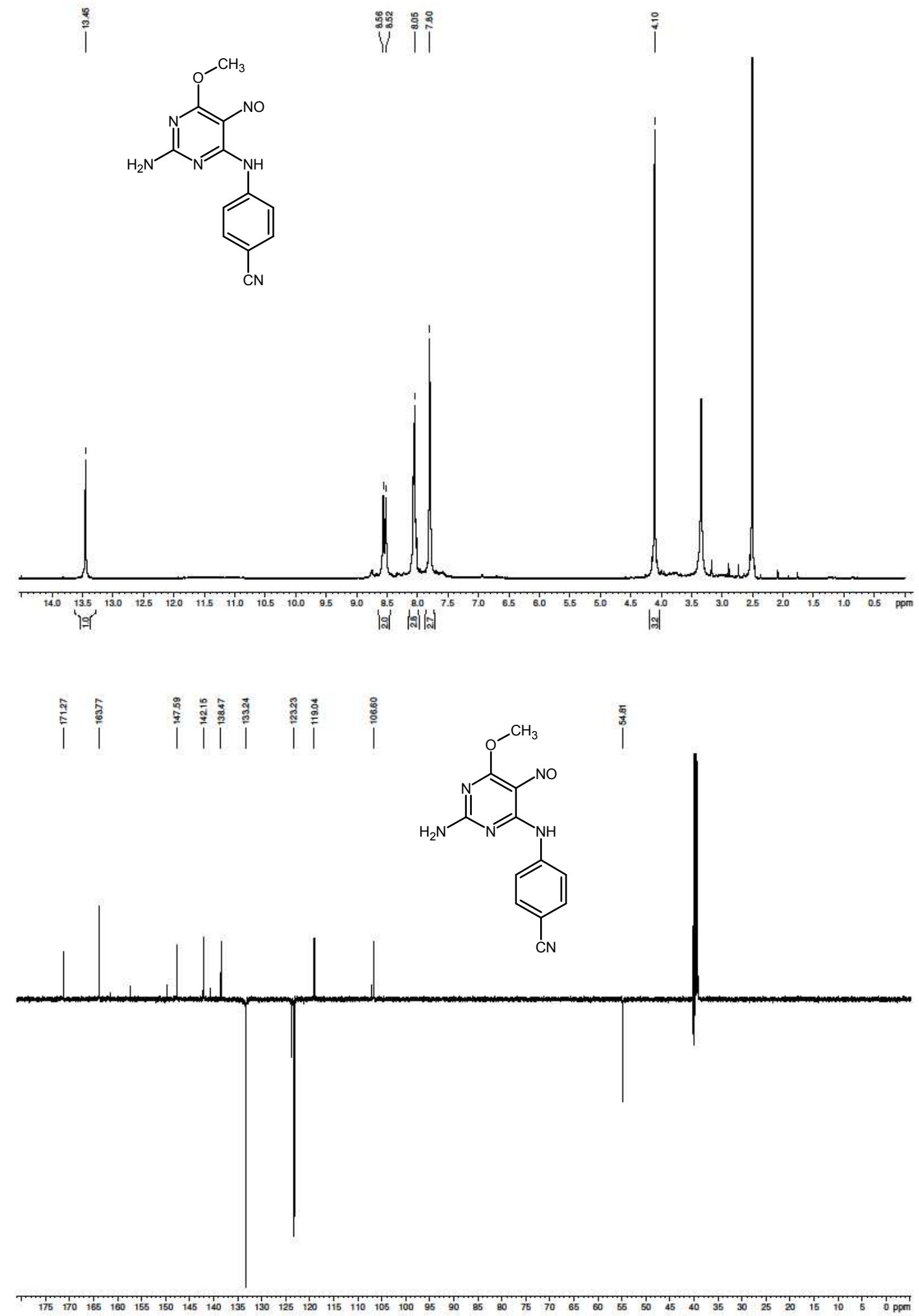

Figure S5. The NMR spectra of compound 3a. 

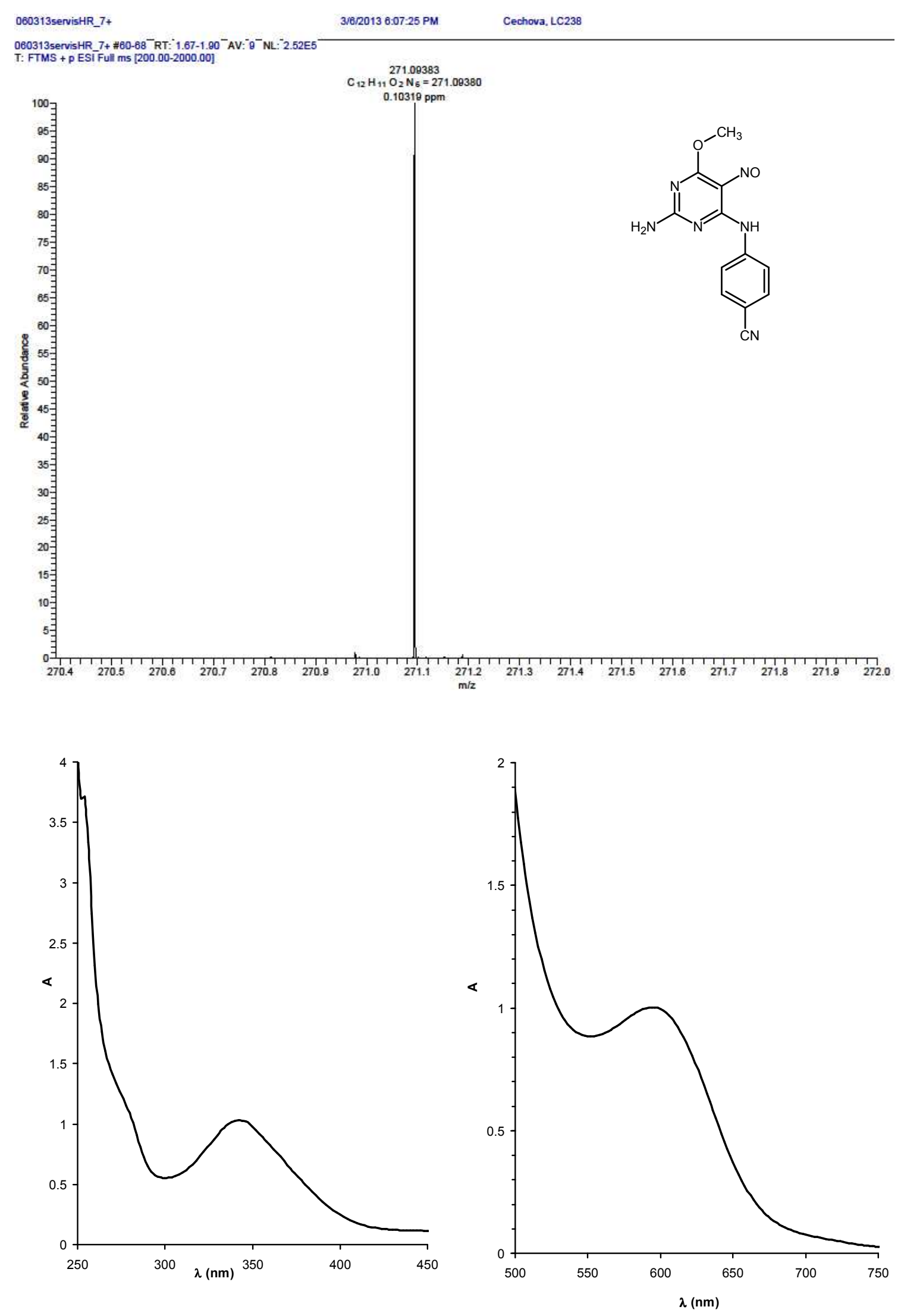

Figure S6. The HR-MS spectrum (top) and electronic spectra (bottom) of compound 3a. 
$i^{8}$<smiles>COc1nc(N)nc(Nc2ccc(C(F)(F)F)cc2)c1N=O</smiles>
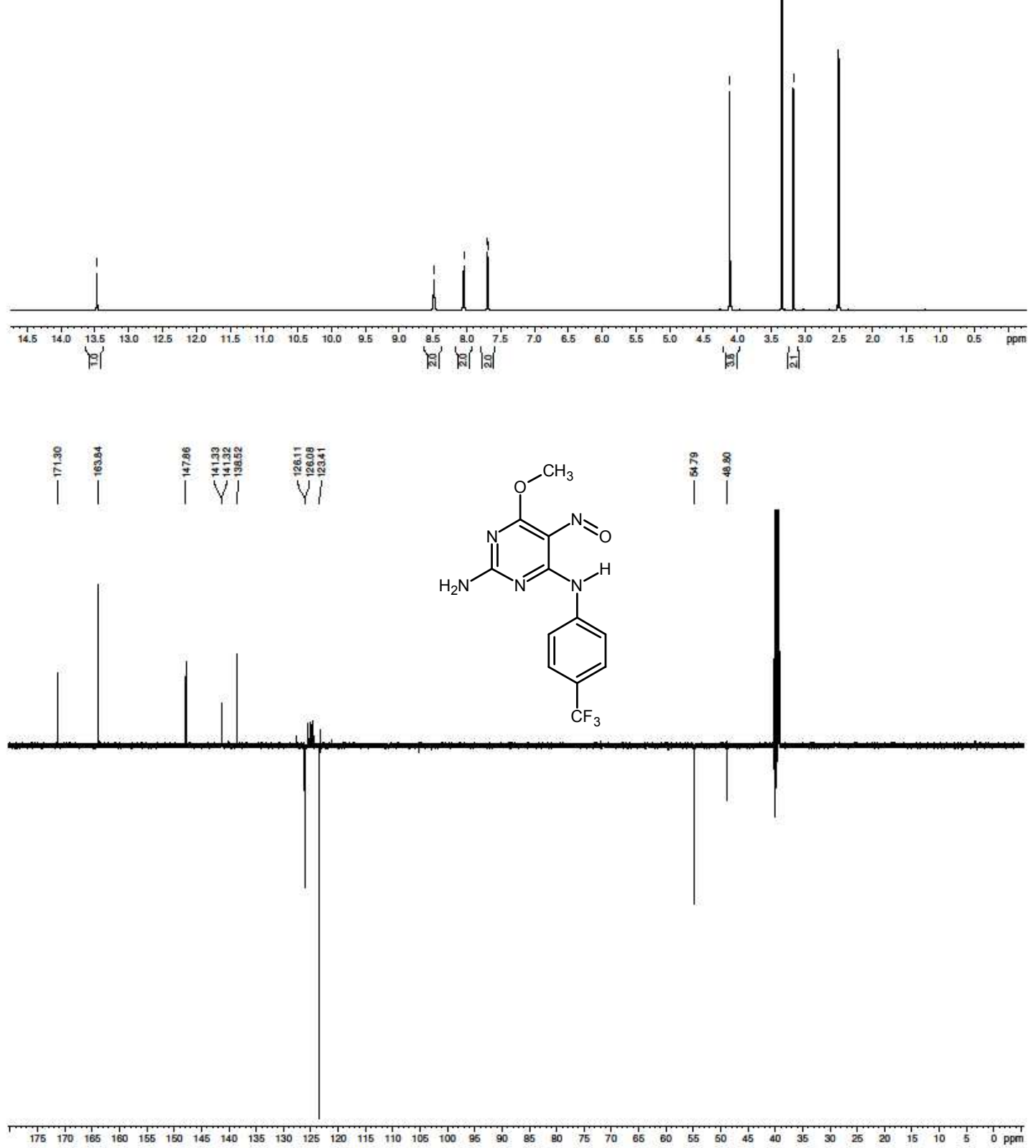

Figure S7. The NMR spectra of the compound 3b. 

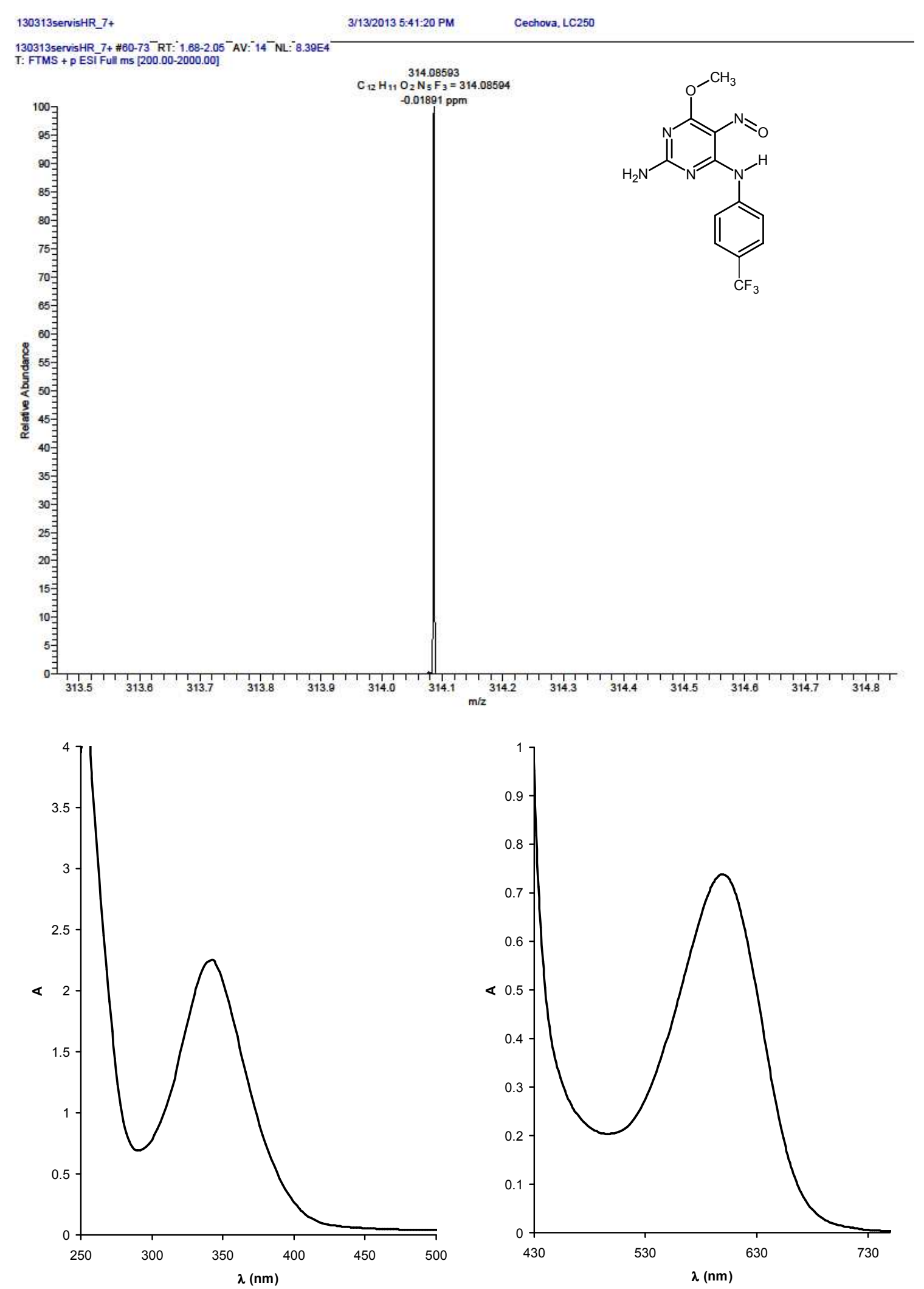

Figure S8. The HR-MS spectrum (top) and electronic spectra (bottom) of compound $\mathbf{3 b}$. 


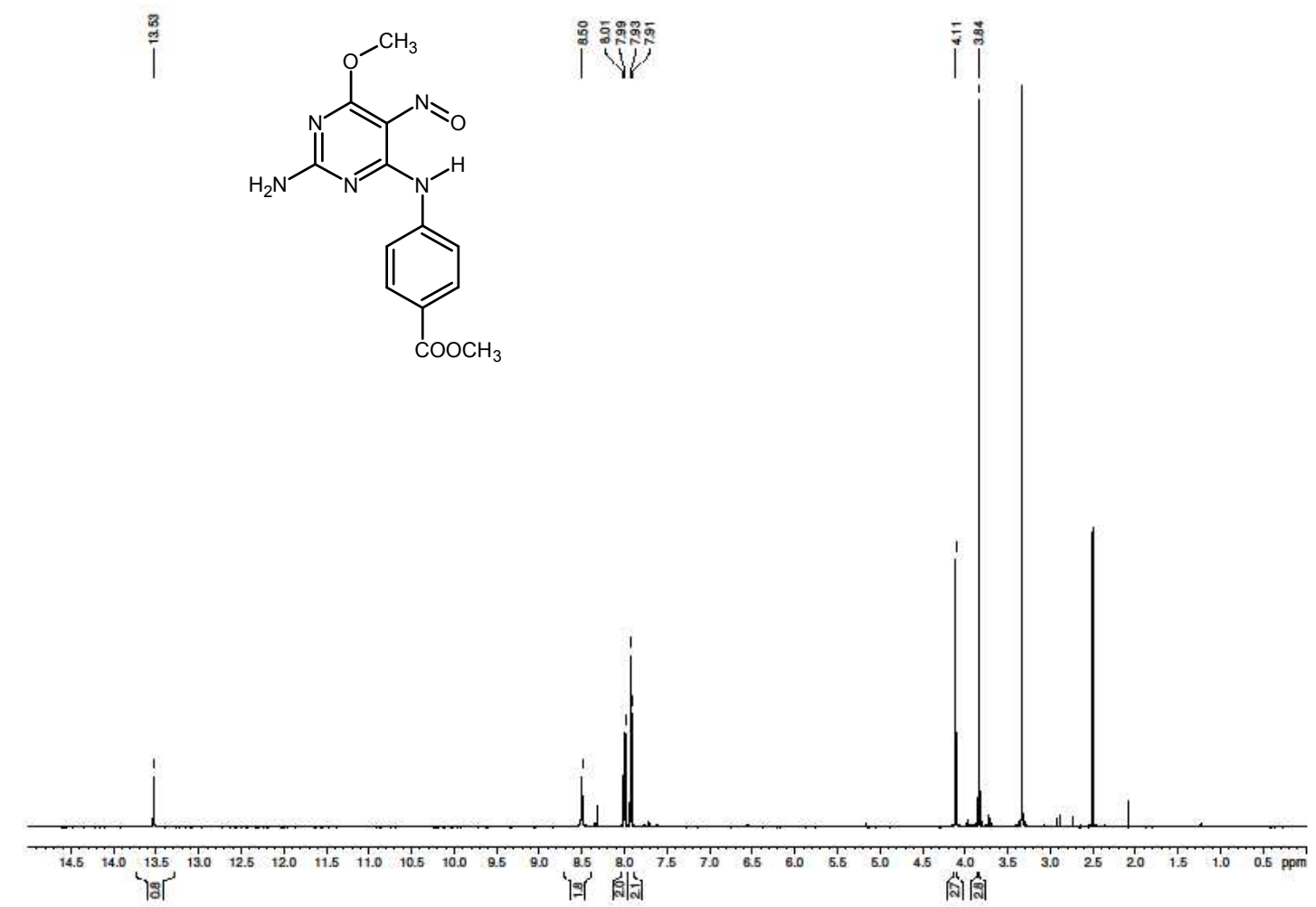

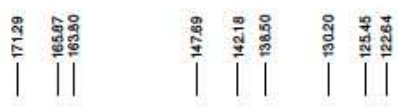
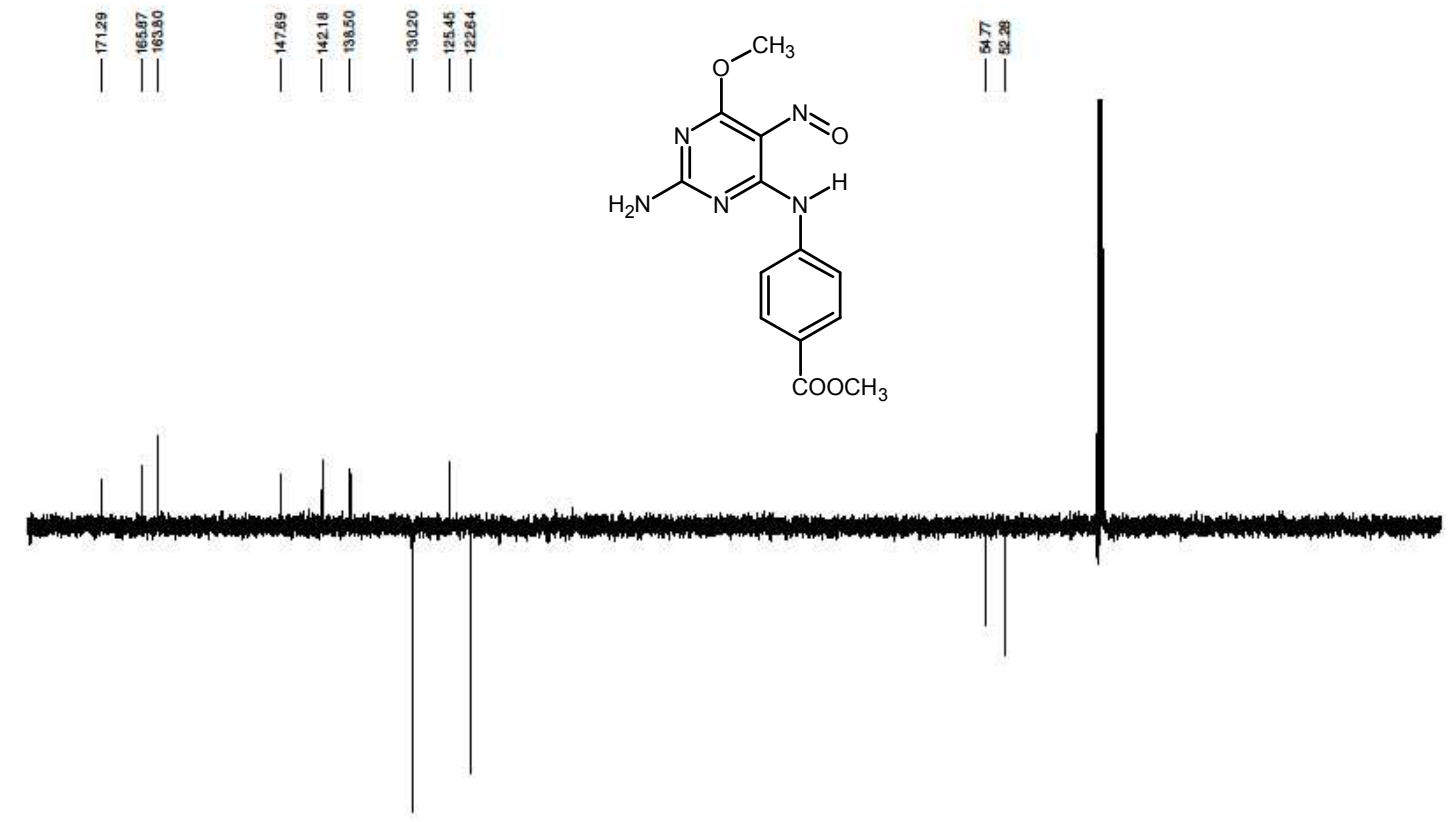

Figure S9. The NMR spectra of compound 3c. 

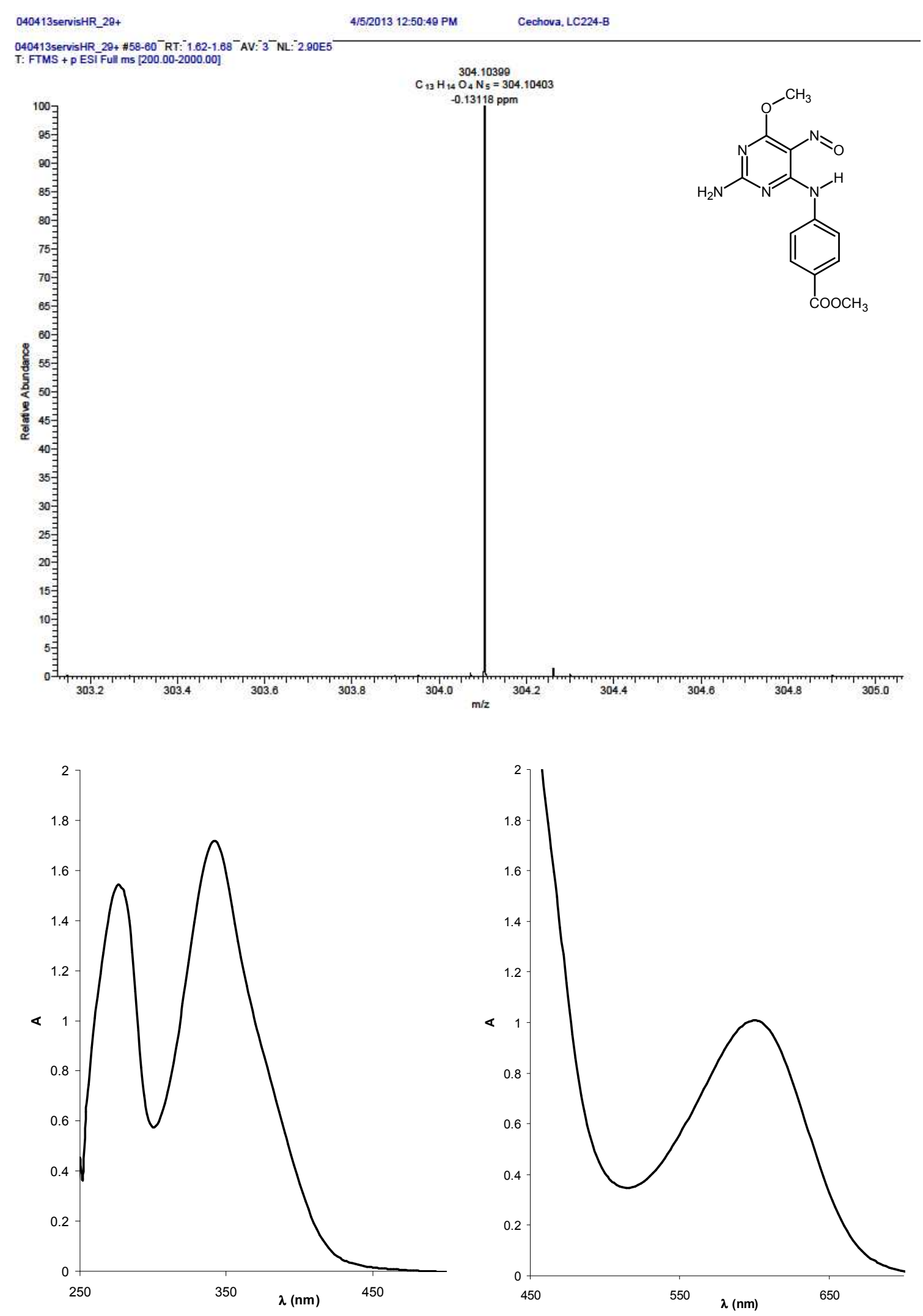

Figure S10. The HR-MS spectrum (top) and electronic spectra (bottom) of compound 3c. 

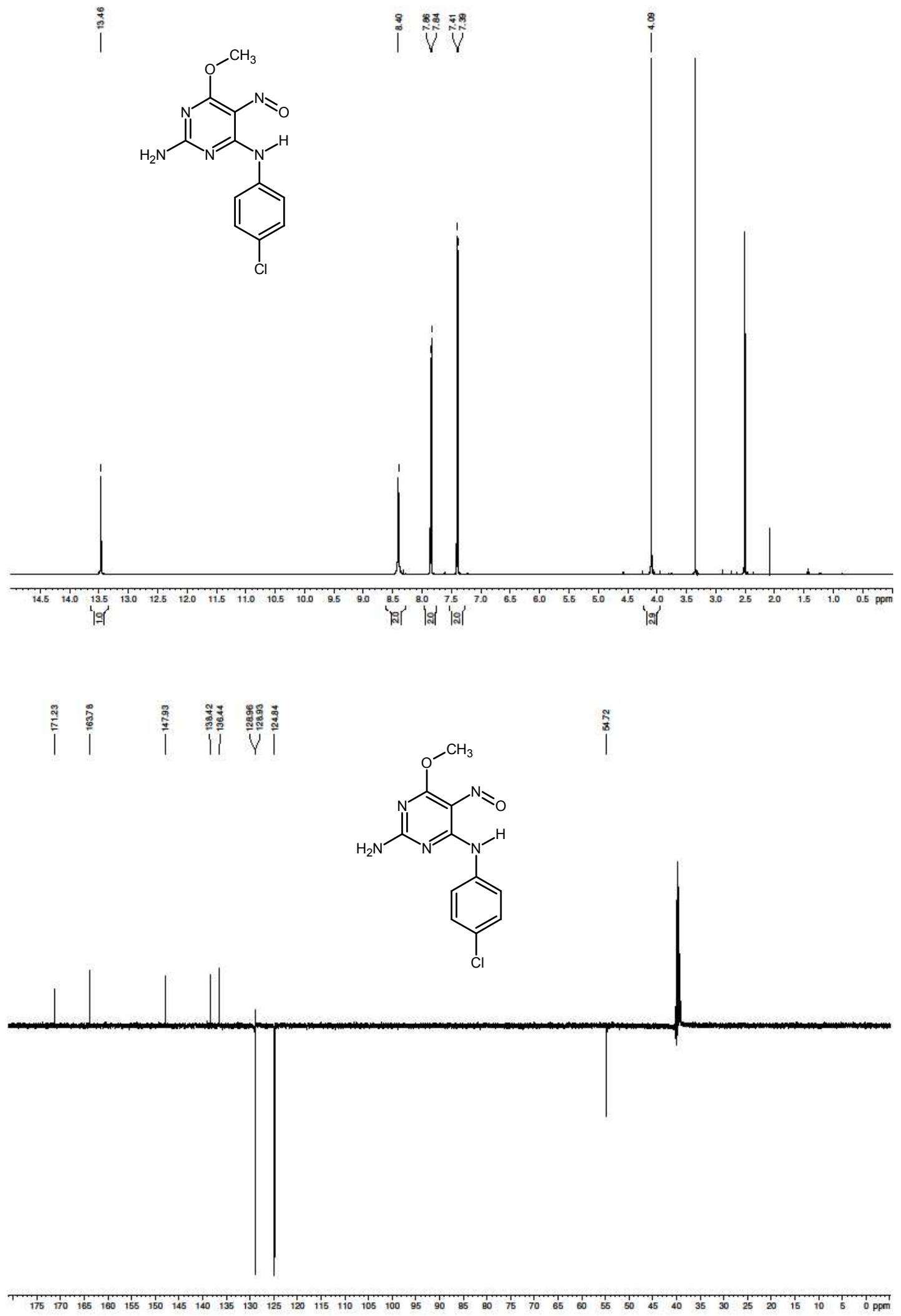

Figure S11. The NMR spectra of compound 3d. 

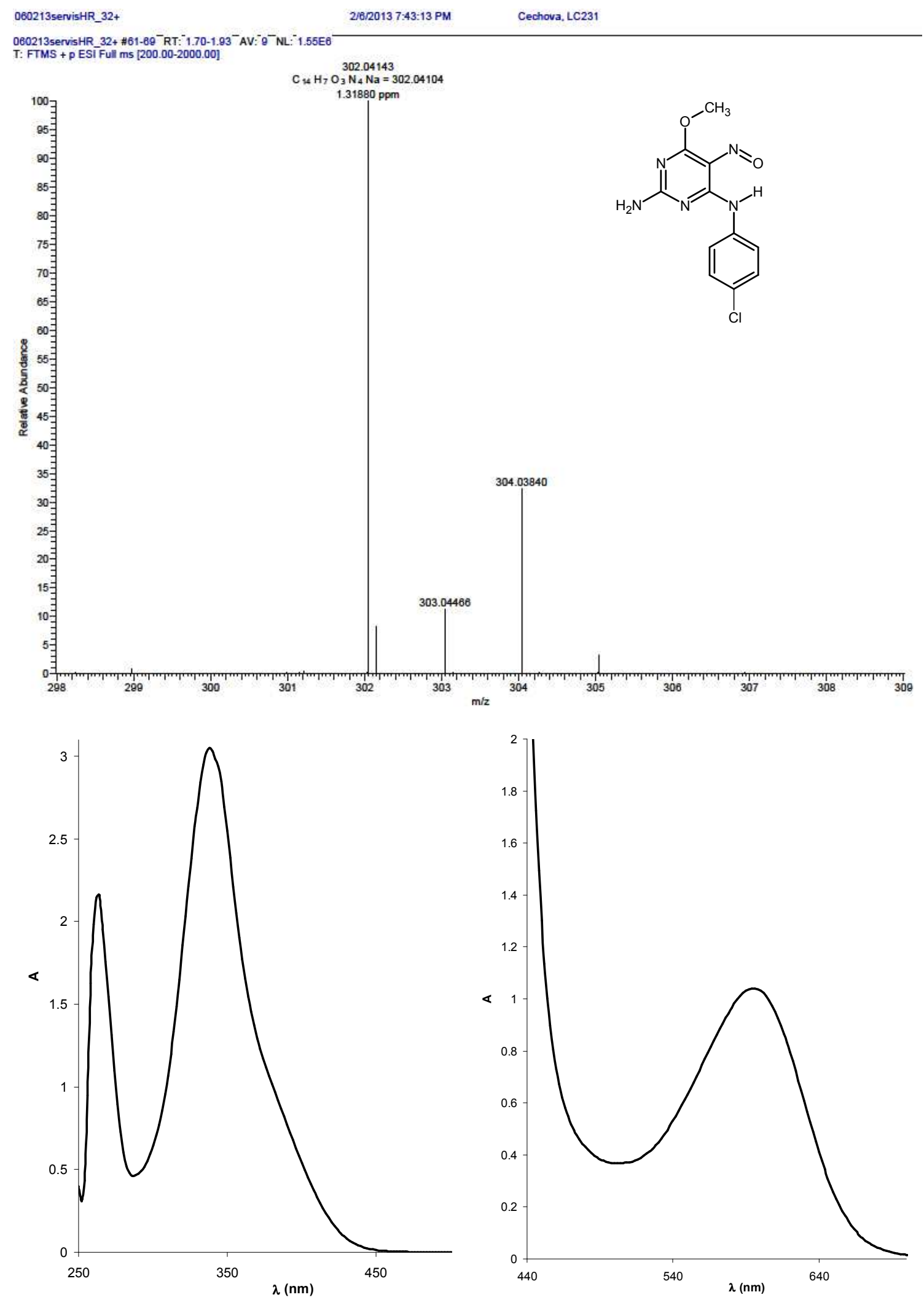

Figure S12. The HR-MS spectrum (top) and electronic spectra (bottom) of compound 3d. 
<smiles>COc1nc(N)nc(Nc2ccccc2)c1N=O</smiles>
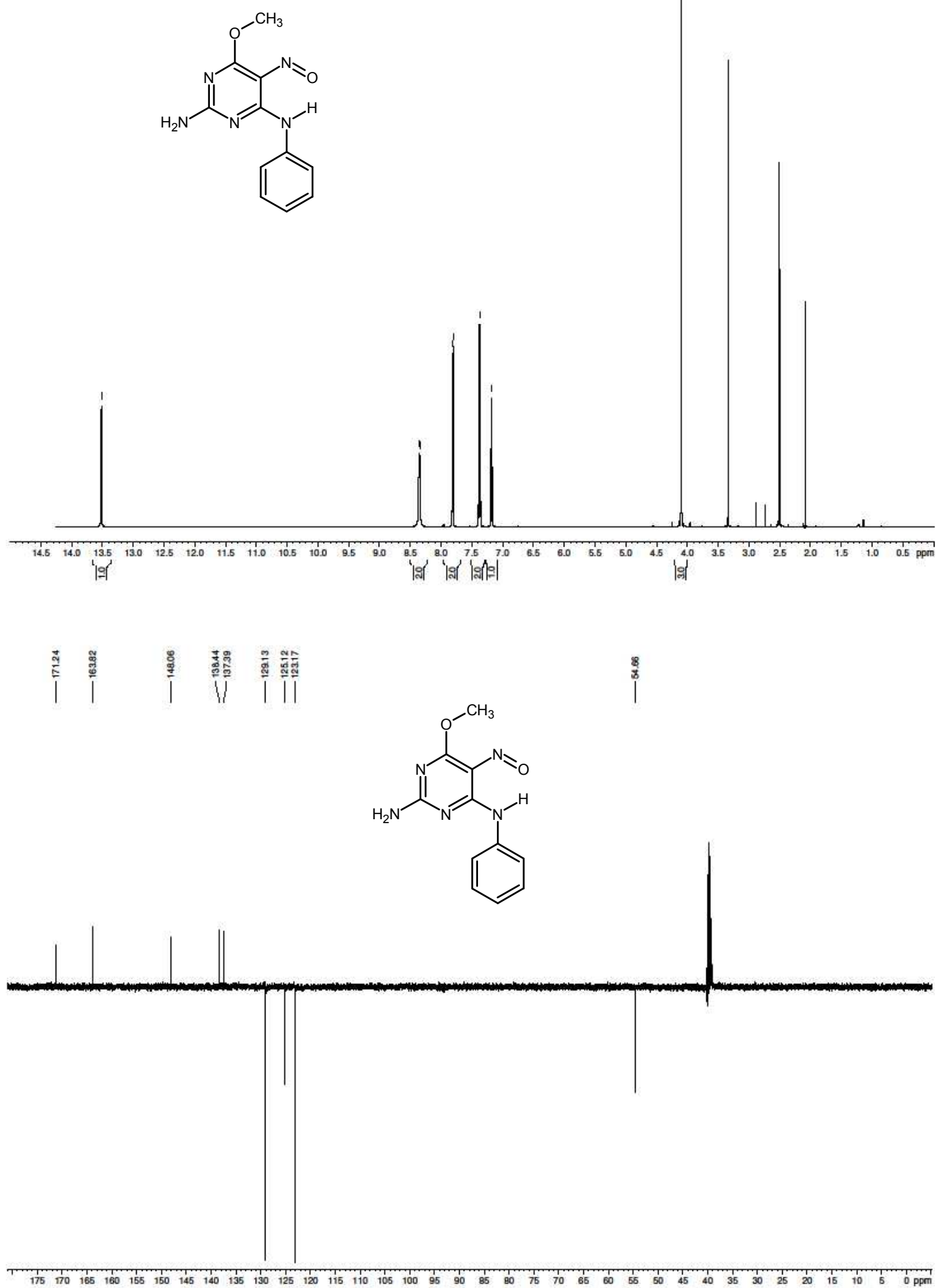

Figure S13. The NMR spectra of compound $3 \mathbf{e}$. 

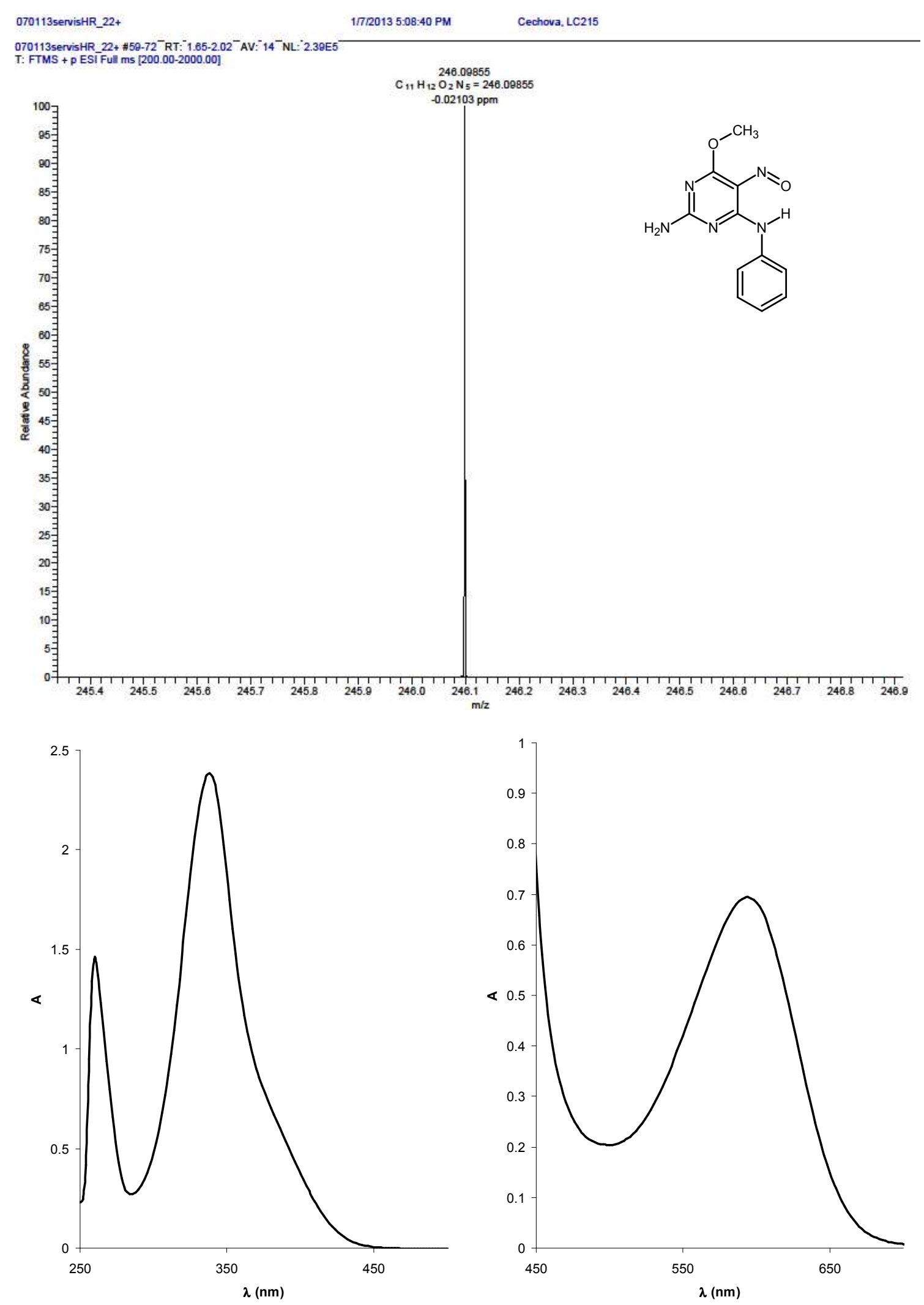

Figure S14. The HR-MS spectrum (top) and electronic spectra (bottom) of compound 3e. 


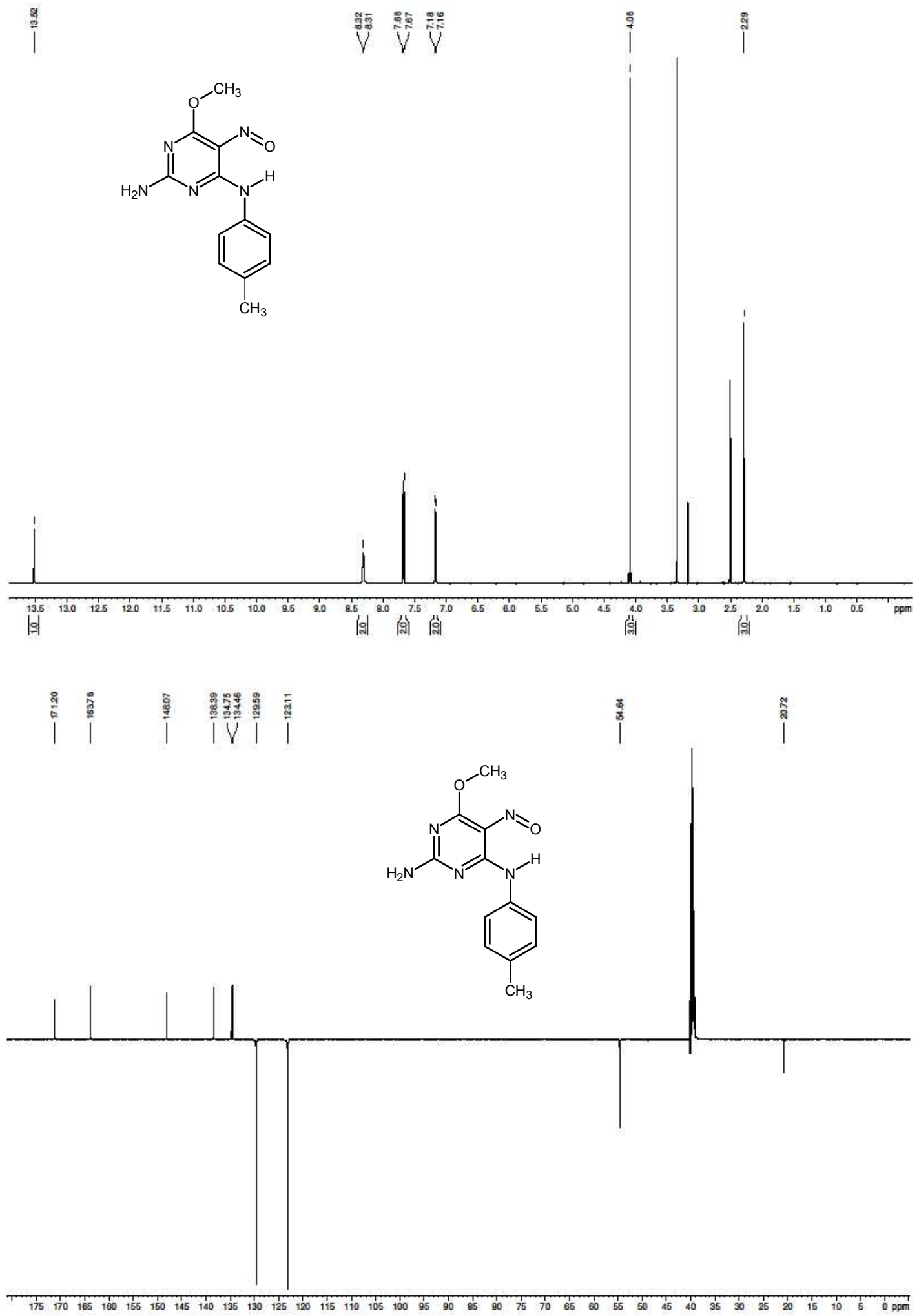

Figure S15. The NMR spectra of compound $\mathbf{3 f}$. 

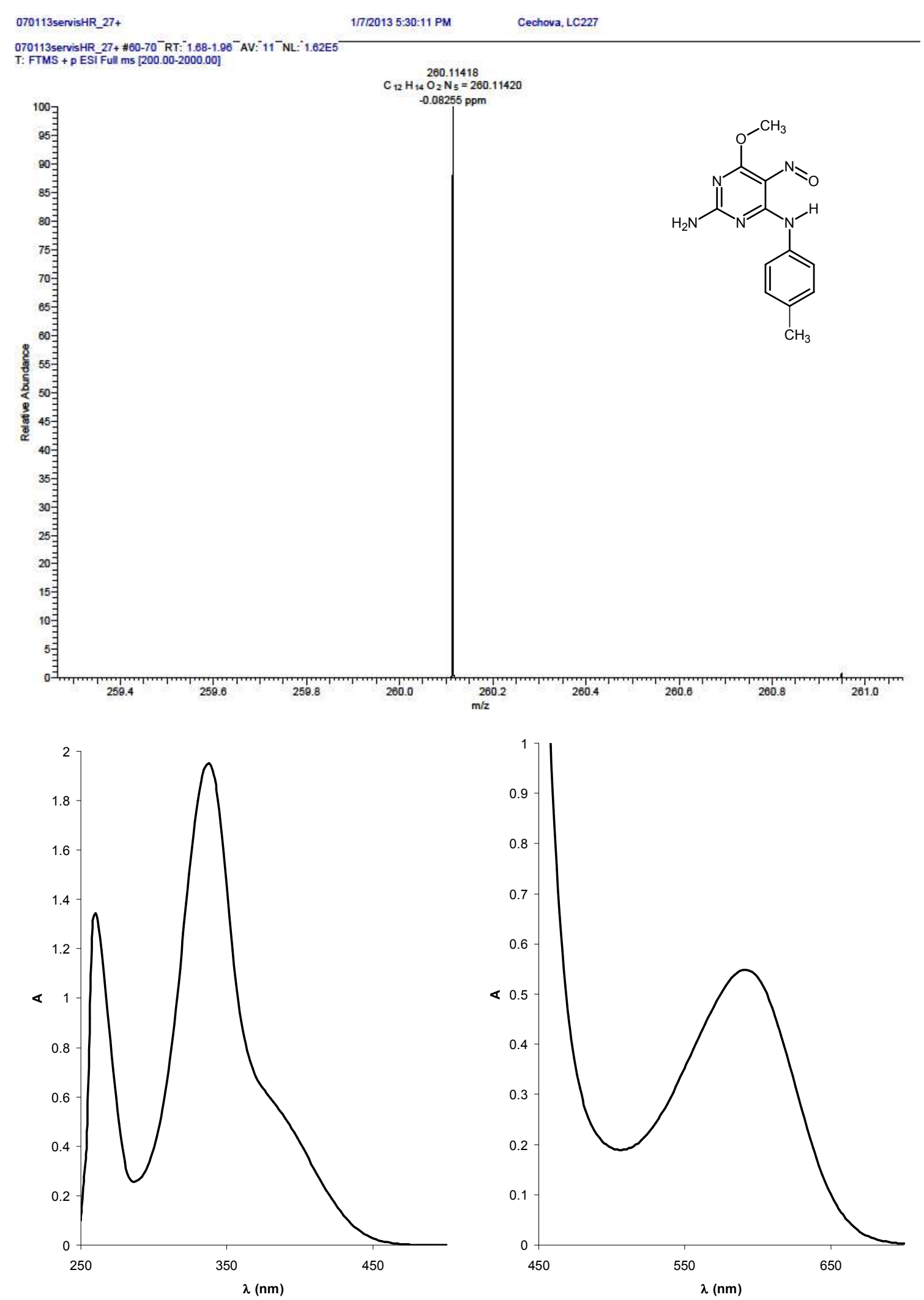

Figure S16. The HR-MS spectrum (top) and electronic spectra (bottom) of compound $\mathbf{3 f}$. 

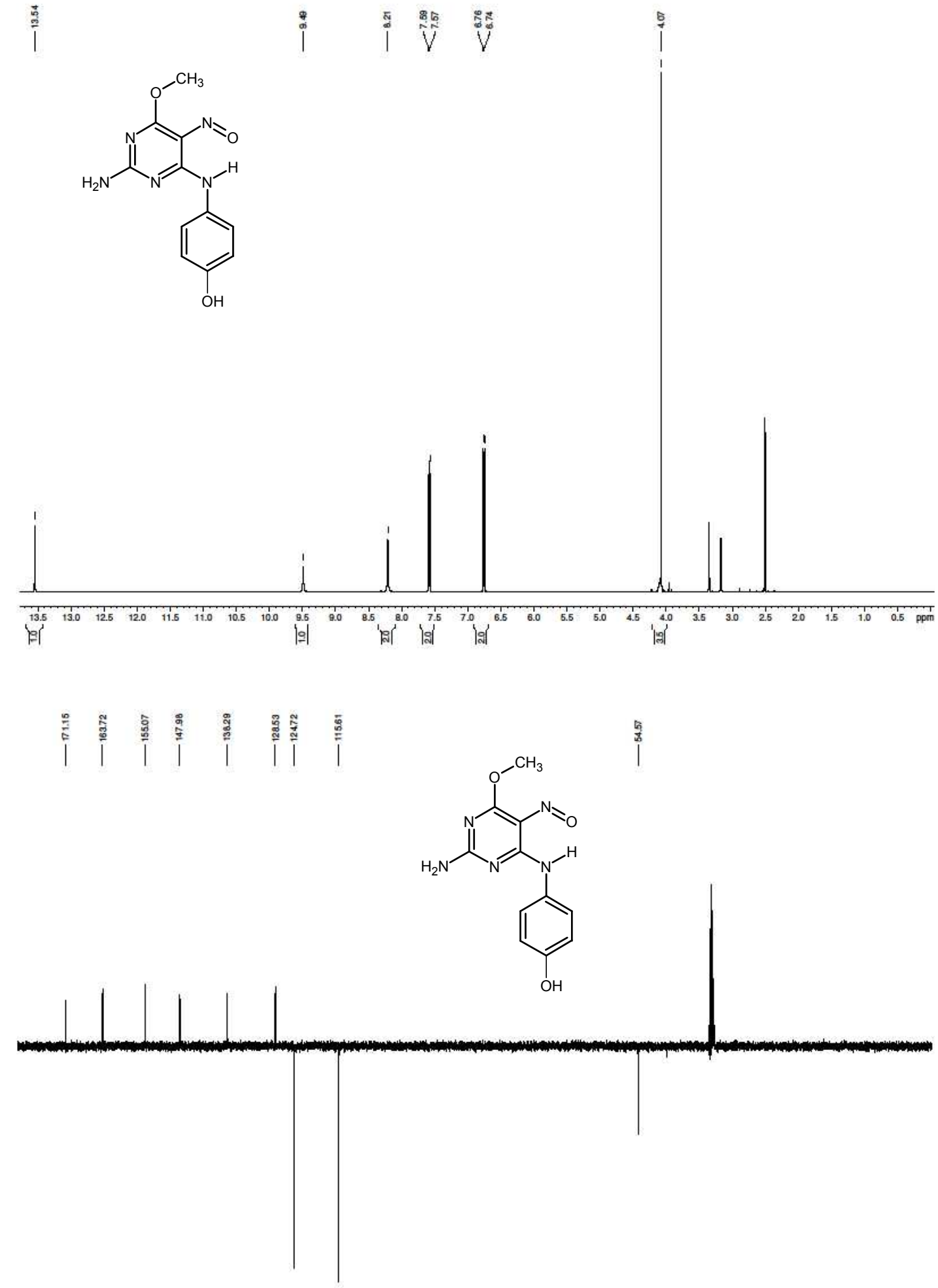

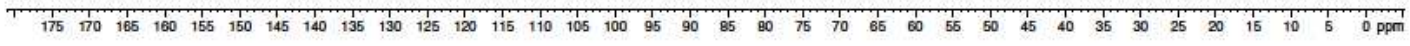

Figure S17. The NMR spectra of compound 3g. 

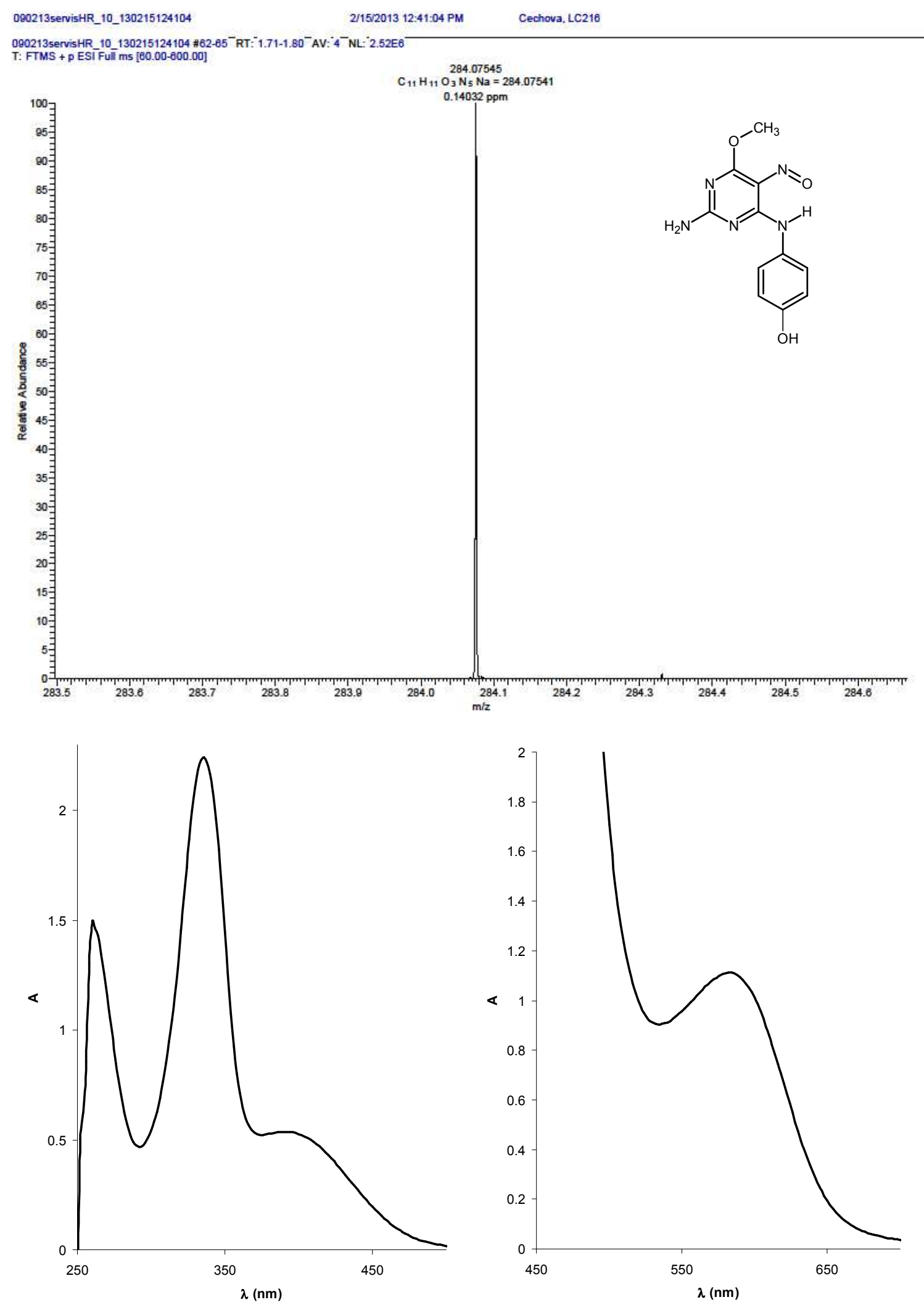

Figure S18. The HR-MS spectrum (top) and electronic spectra (bottom) of compound 3g. 


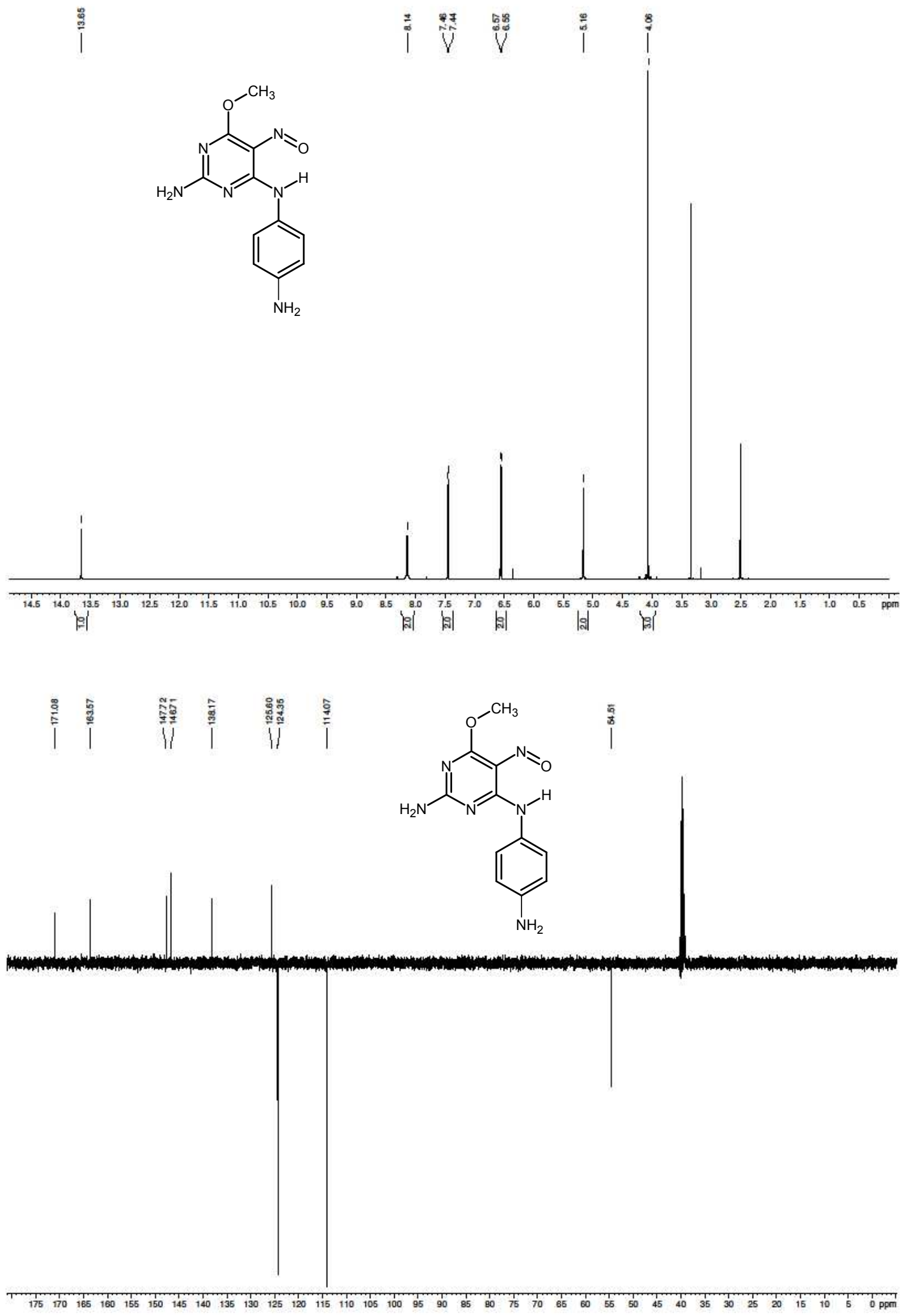

Figure S19. The NMR spectra of compound $\mathbf{3 h}$. 

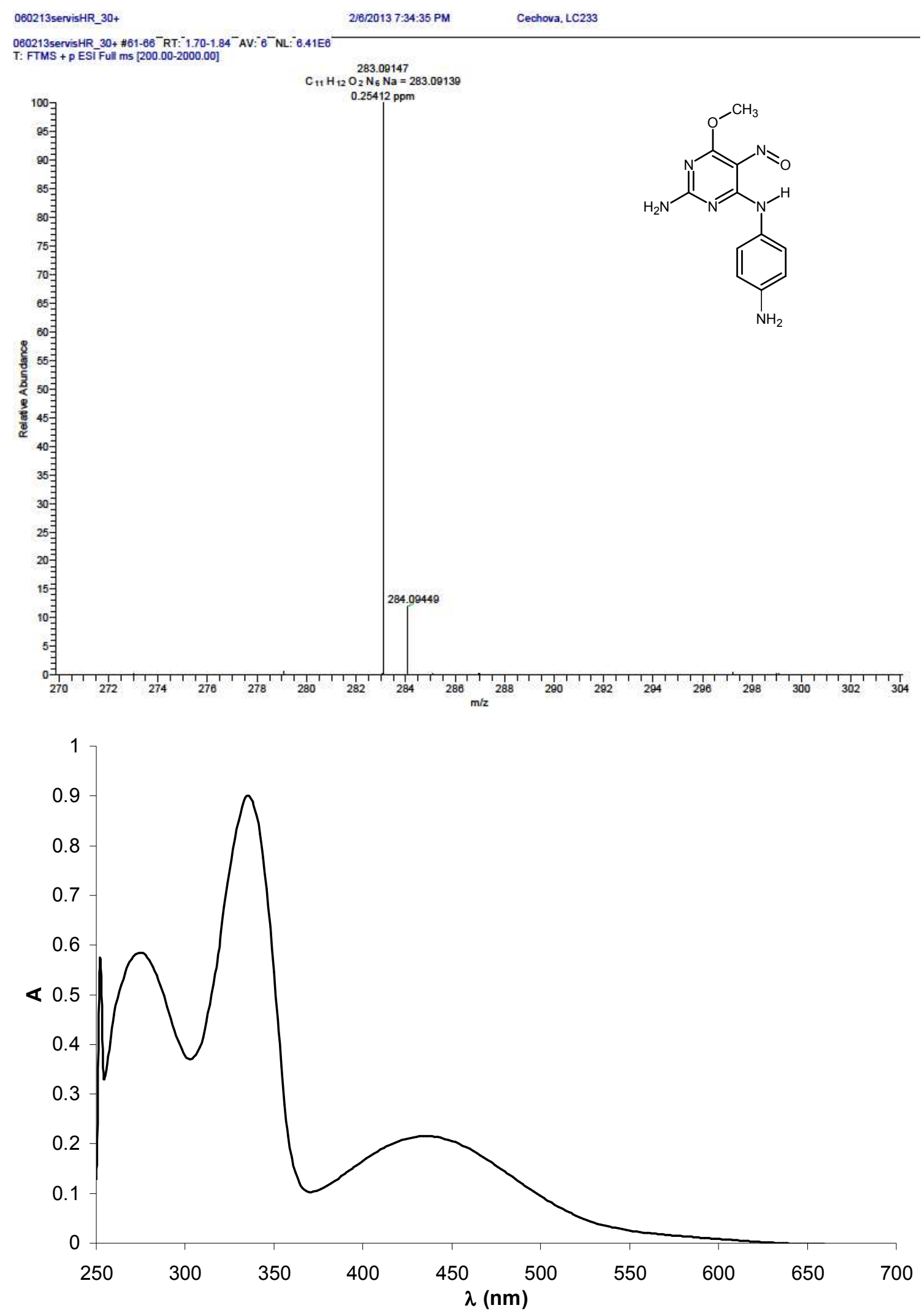

Figure S20. The HR-MS spectrum (top) and electronic spectra (bottom) of compound $\mathbf{3 h}$. 


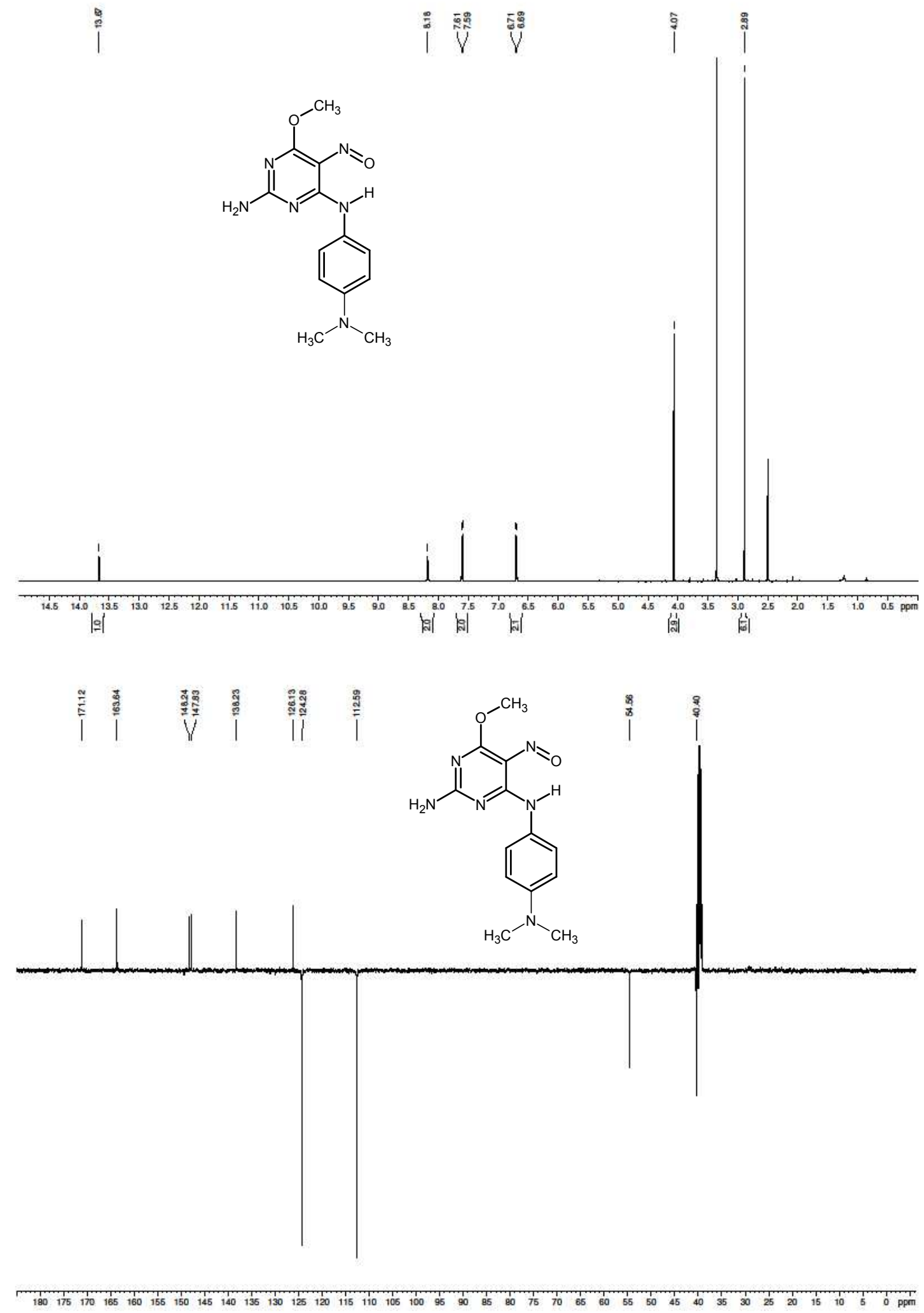

Figure S21. The NMR spectra of compound 3i. 

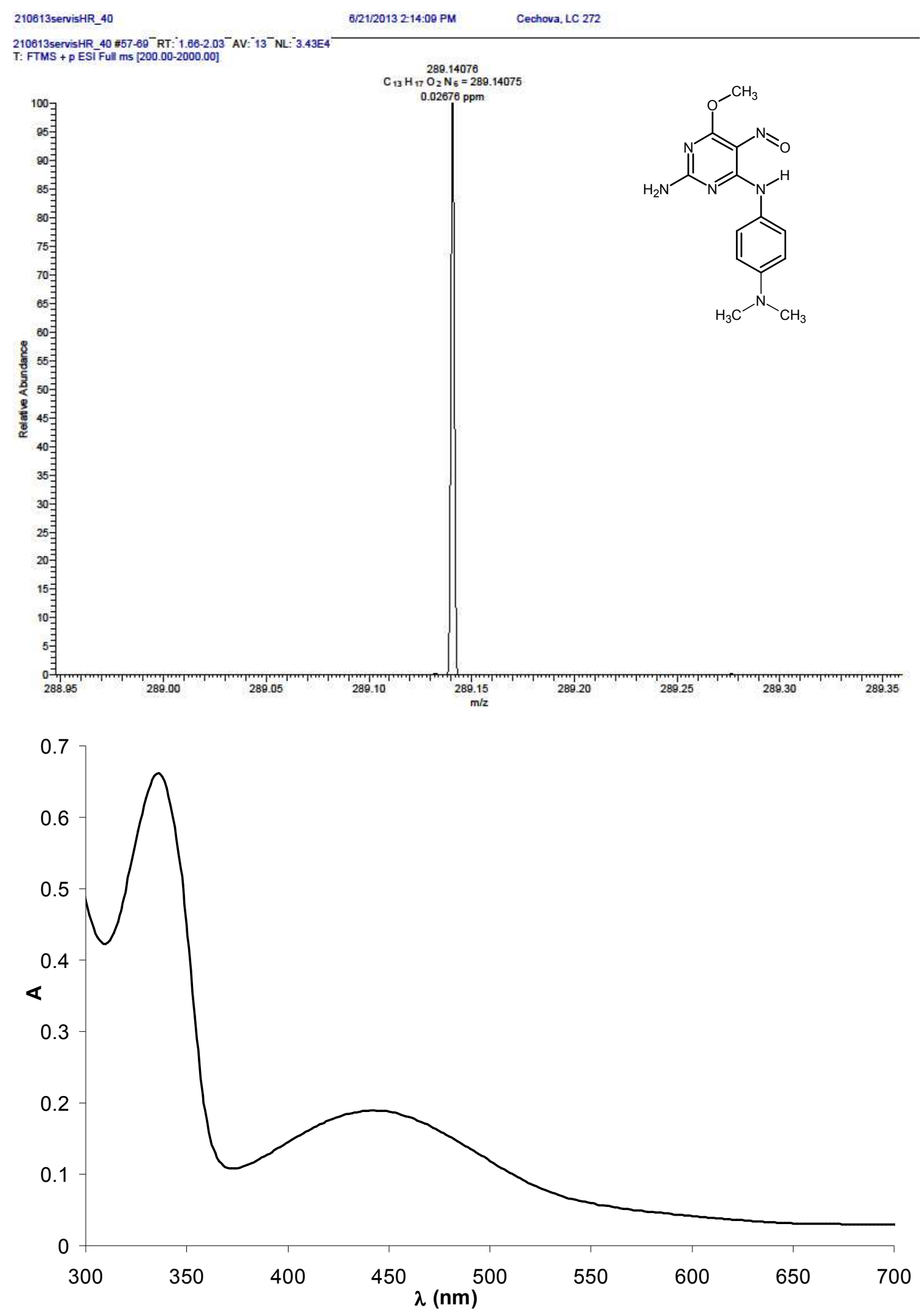

Figure S22. The HR-MS spectrum (top) and electronic spectra (bottom) of compound $3 \mathbf{i}$. 


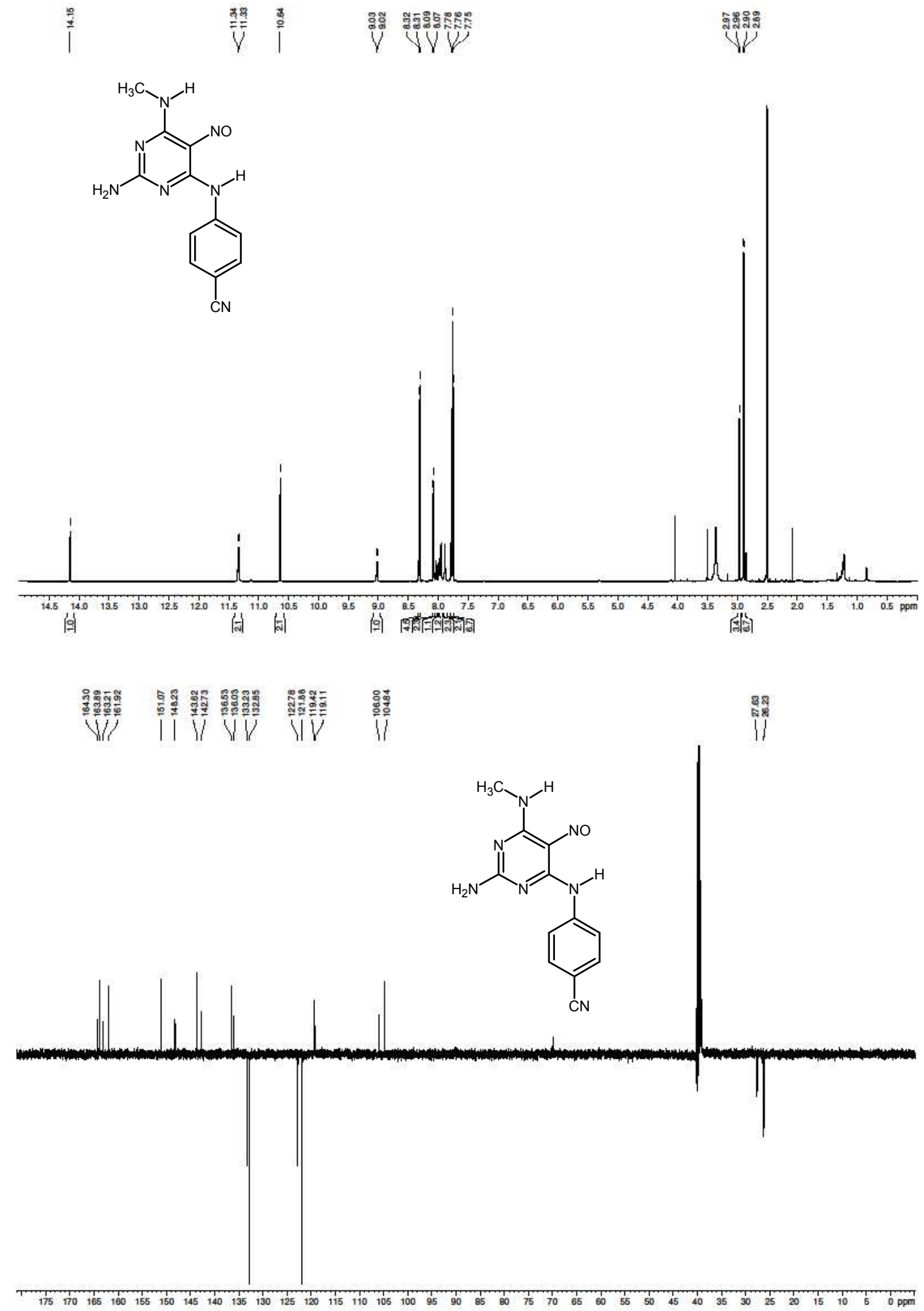

Figure S23. The NMR spectra of compound $4 \mathbf{a}$. 

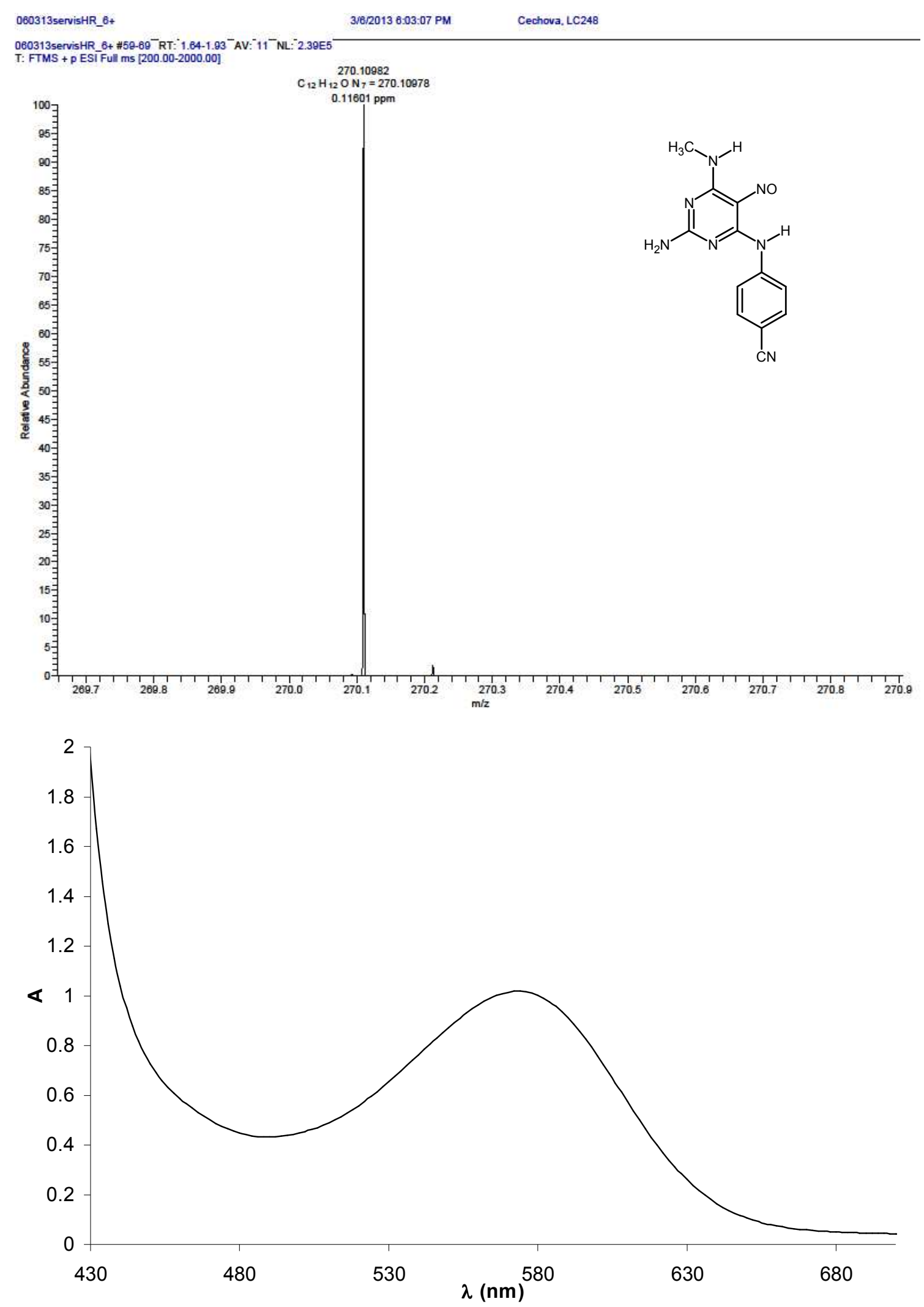

Figure S24. The HR-MS spectrum (top) and electronic spectrum (bottom) of compound $4 \mathbf{a}$. 
I<smiles>CNc1nc(N)nc(Nc2ccc(C(F)(F)F)cc2)c1[N+]#N</smiles>
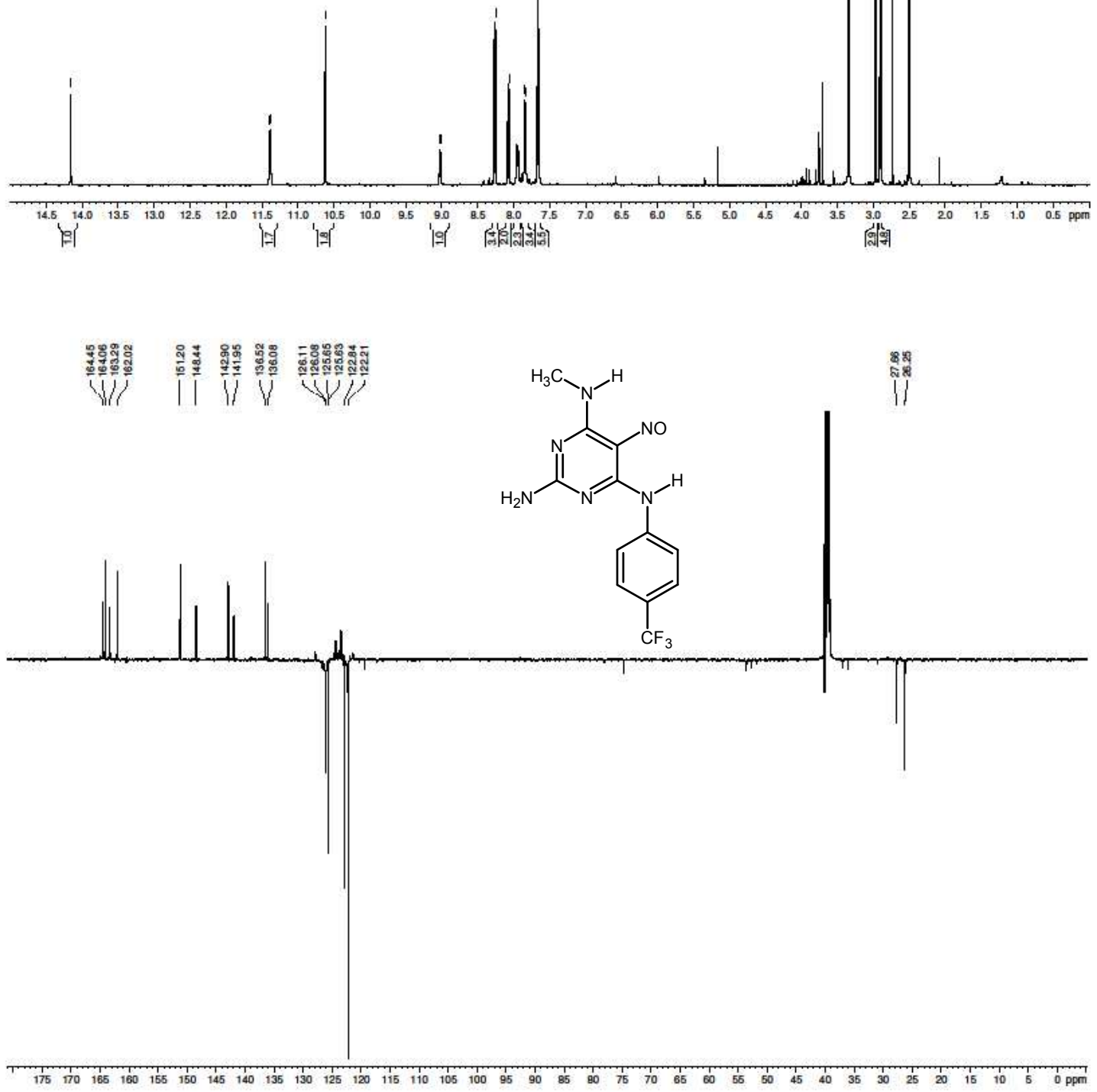

Figure S25. The NMR spectra of compound $\mathbf{4 b}$. 

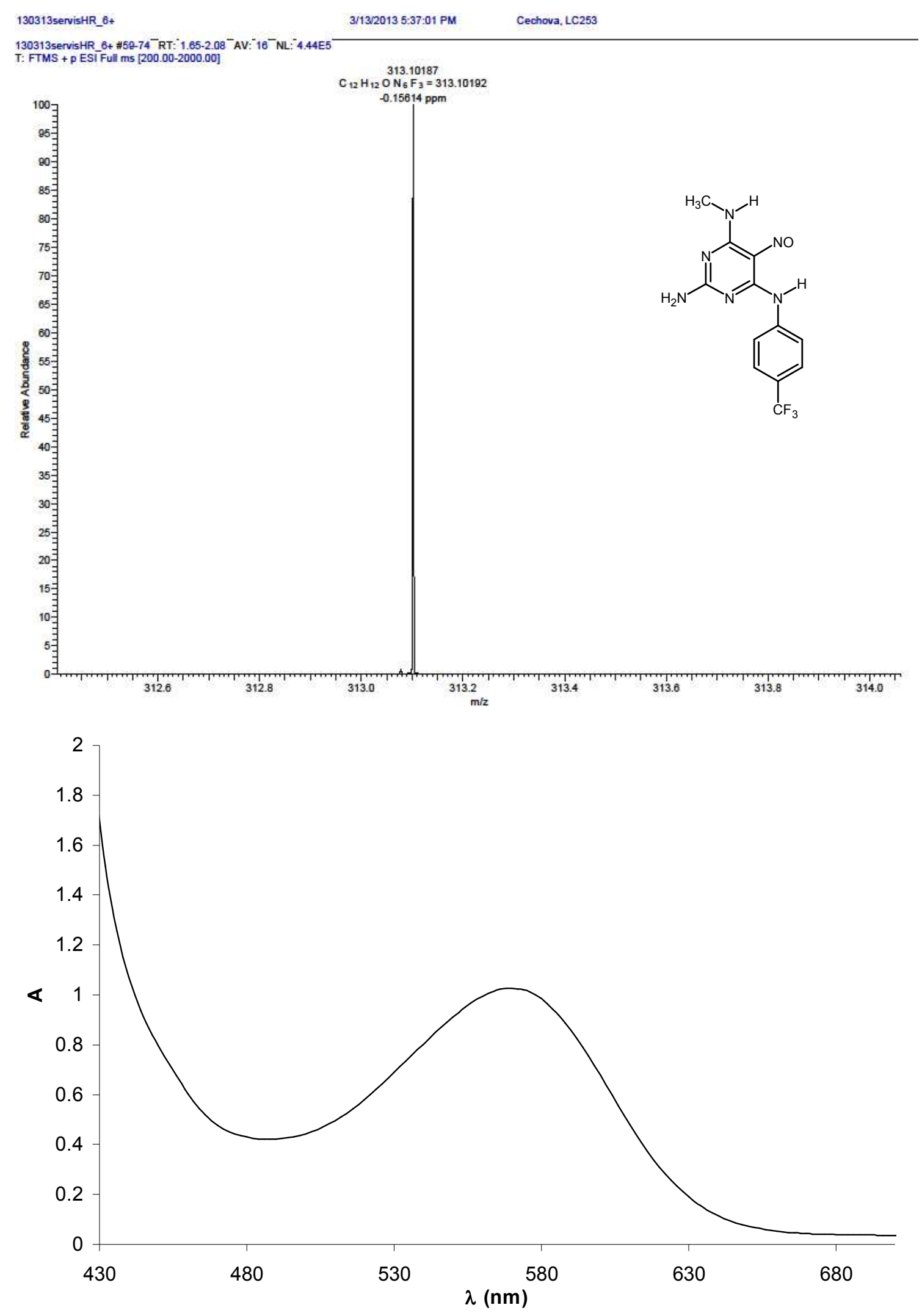

Figure S26. The HR-MS spectrum (top) and electronic spectrum (bottom) of compound $\mathbf{4 b}$. 
$\left.\right|^{\infty}$

$\stackrel{8}{=} \stackrel{8}{=}$

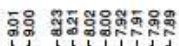

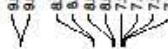<smiles>CNc1nc(N)nc(Nc2ccc(C(C)=O)cc2)c1[N+](=O)[O-]</smiles>
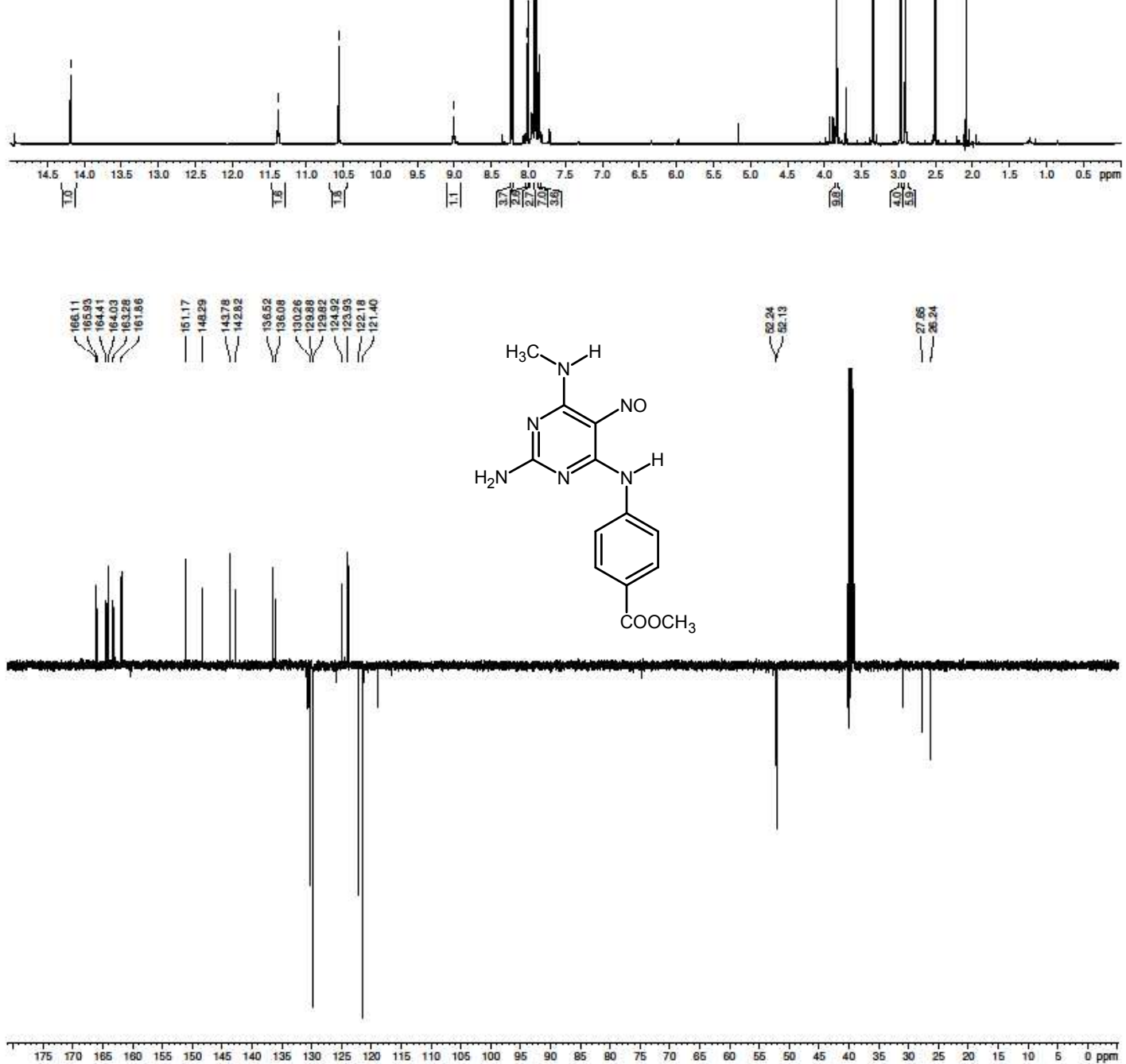

Figure S27. The NMR spectra of compound 4c. 

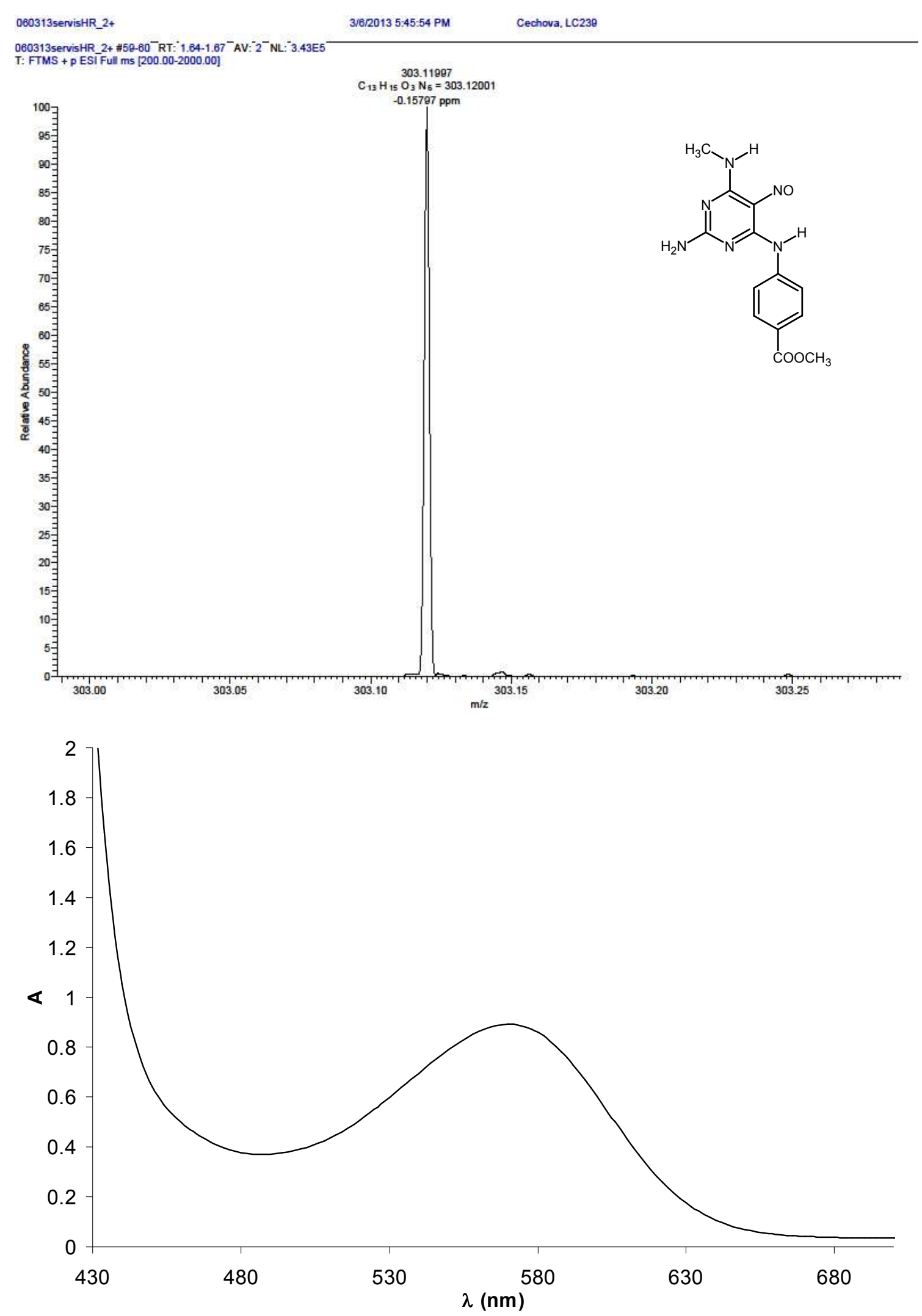

Figure S28. The HR-MS spectrum (top) and electronic spectrum (bottom) of compound 4c. 

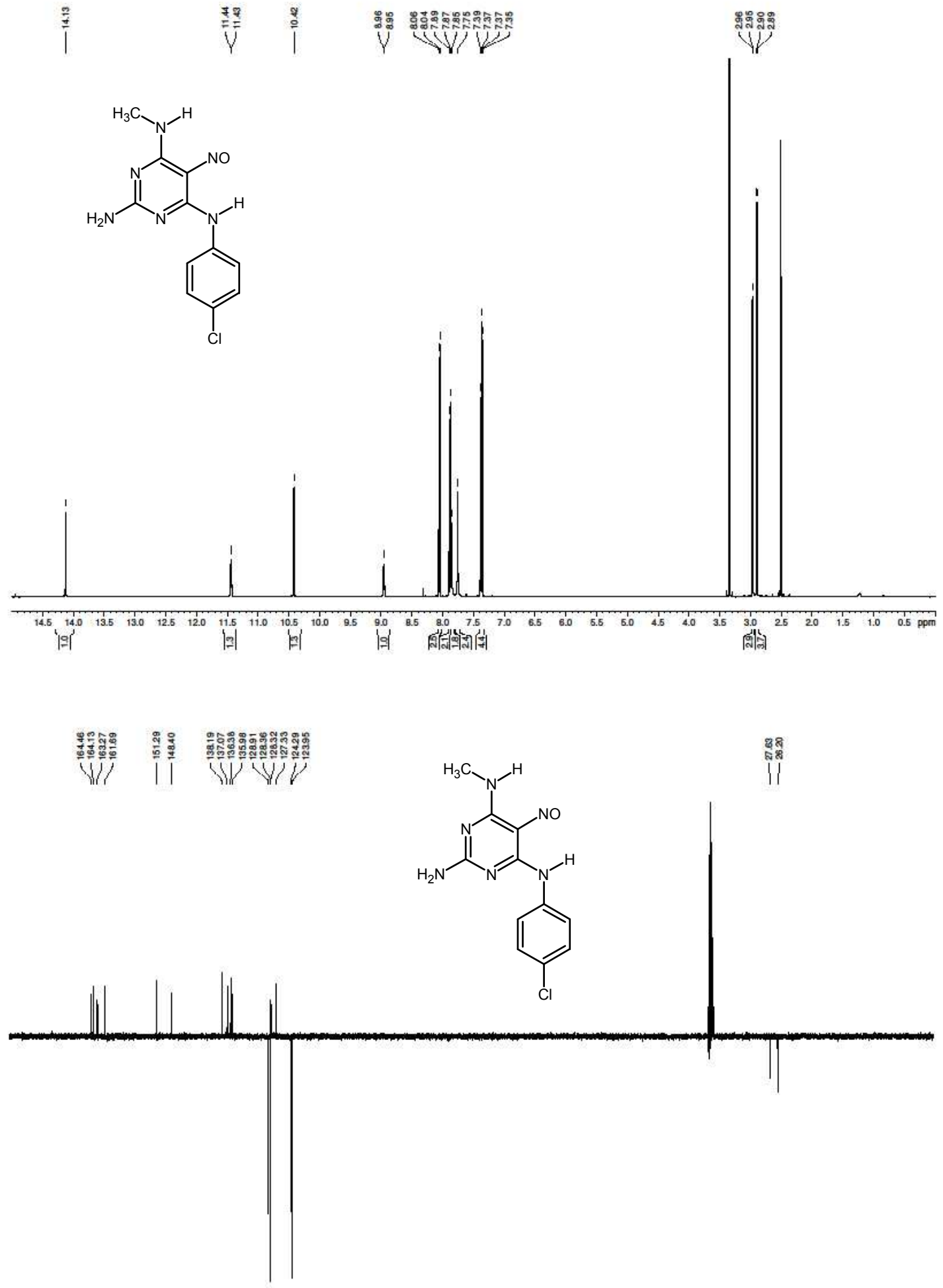

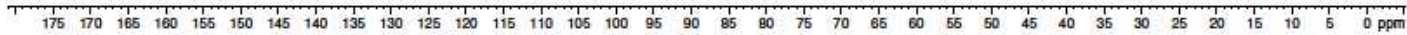

Figure S29. The NMR spectra of compound 4d. 


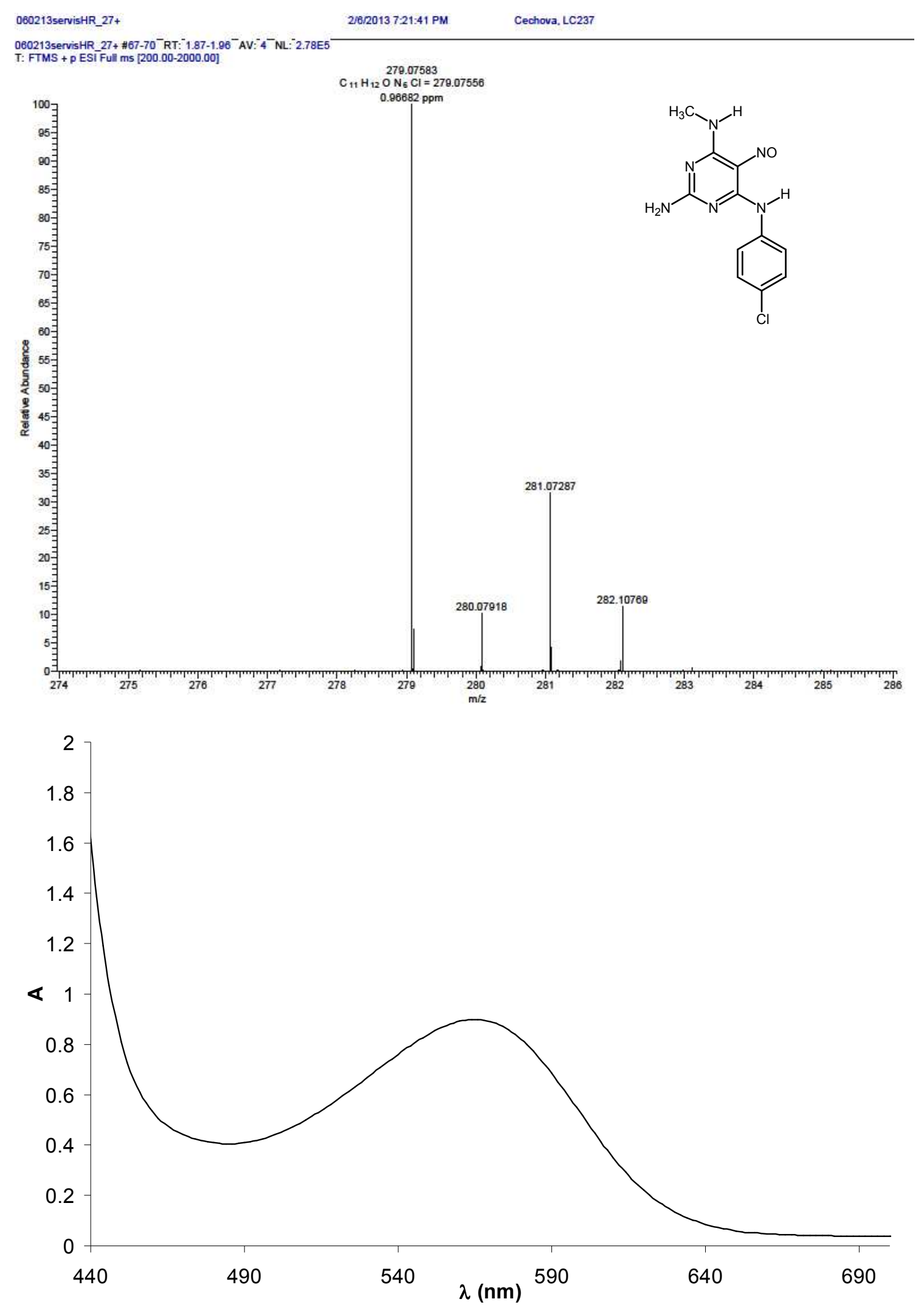

Figure S30. The HR-MS spectrum (top) and electronic spectrum (bottom) of compound 4d. 

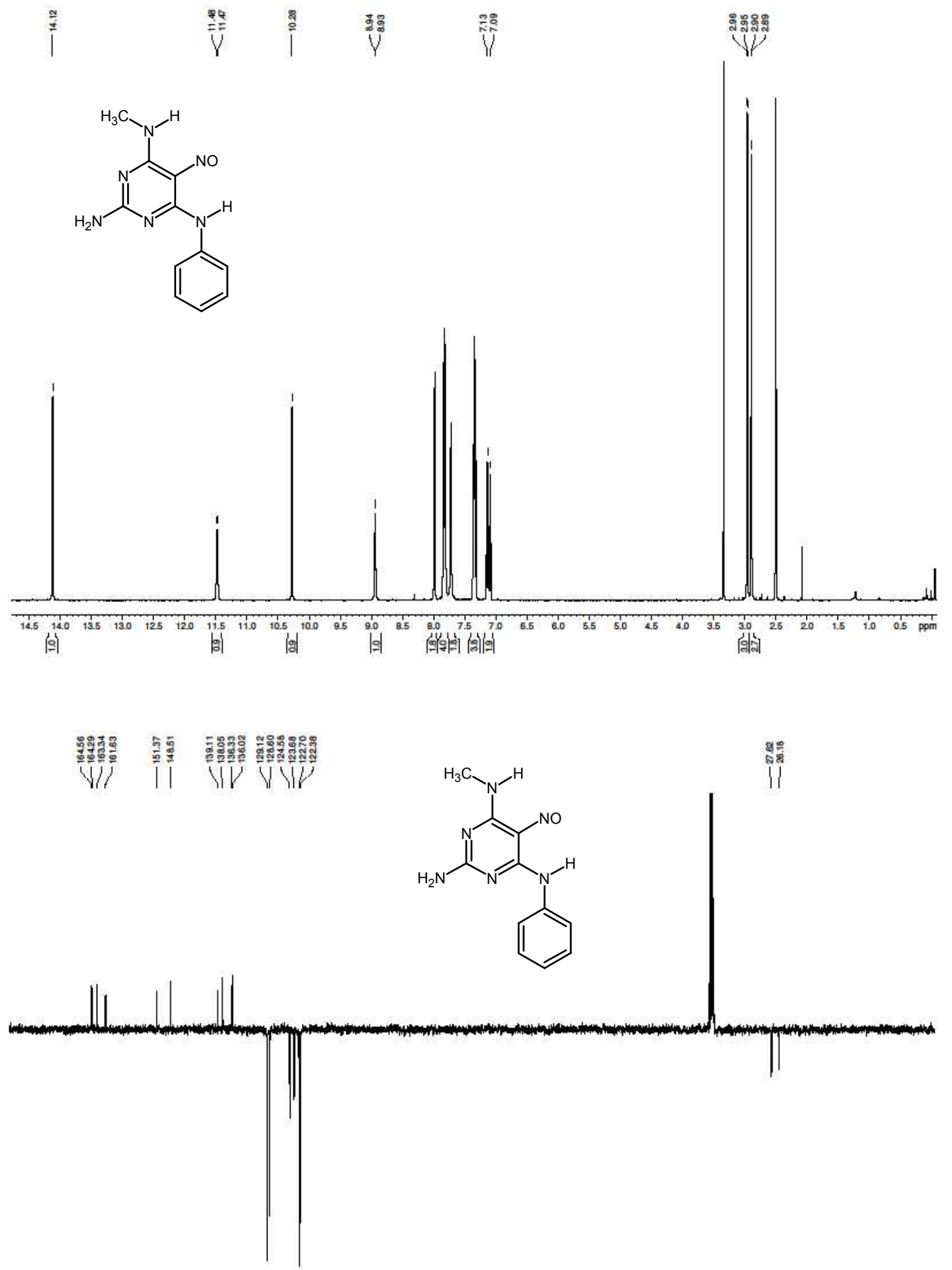

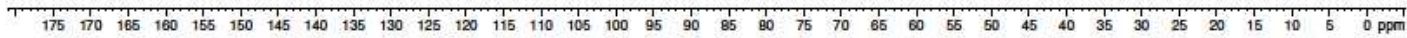

Figure S31. The NMR spectra of compound 4e. 

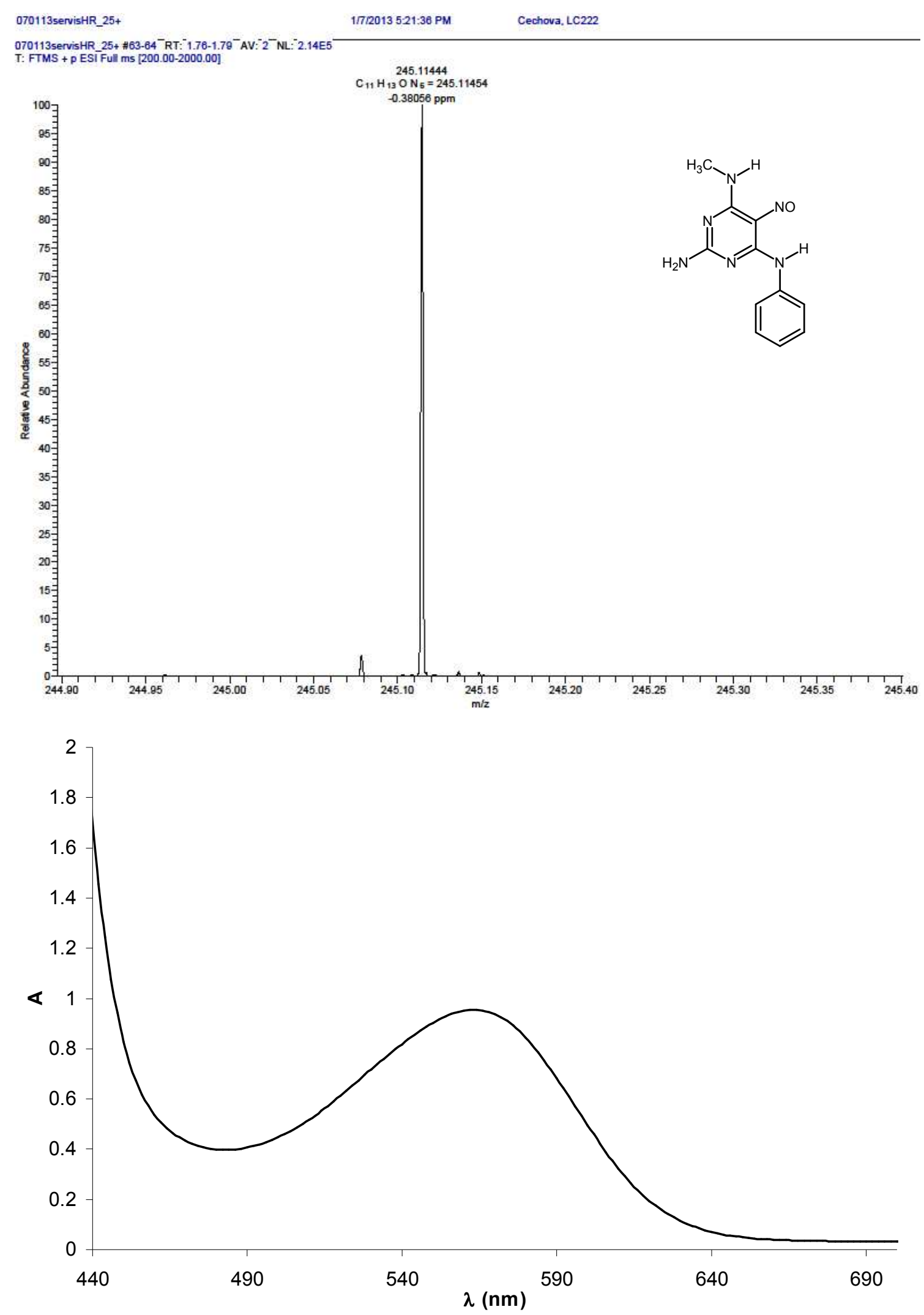

Figure S32. The HR-MS spectrum (top) and electronic spectrum (bottom) of compound $4 \mathbf{e}$. 


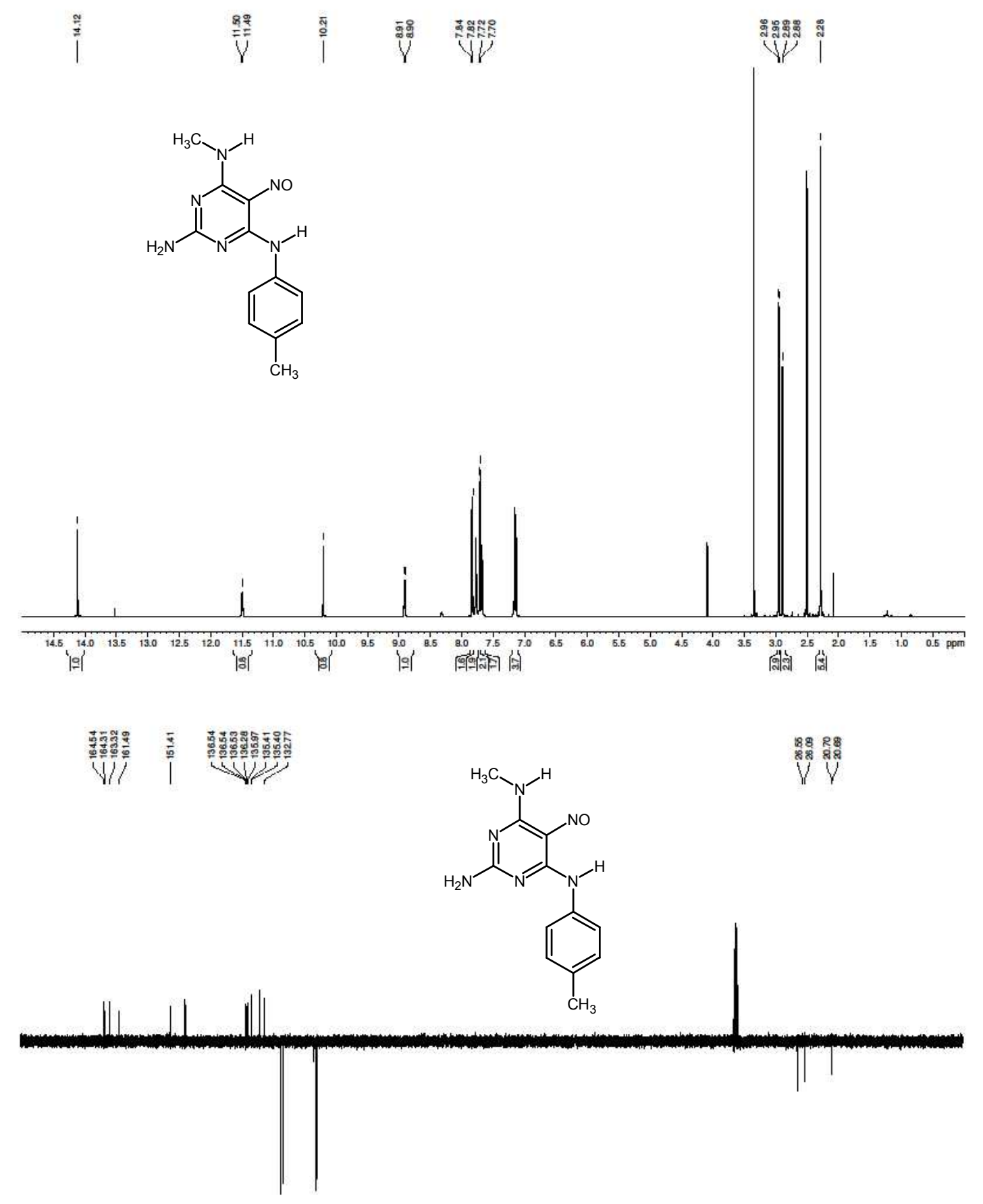

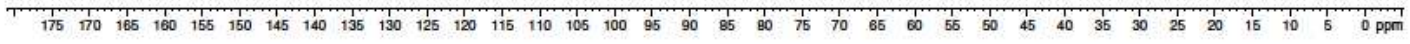

Figure S33. The NMR spectra of compound $\mathbf{4 f}$. 

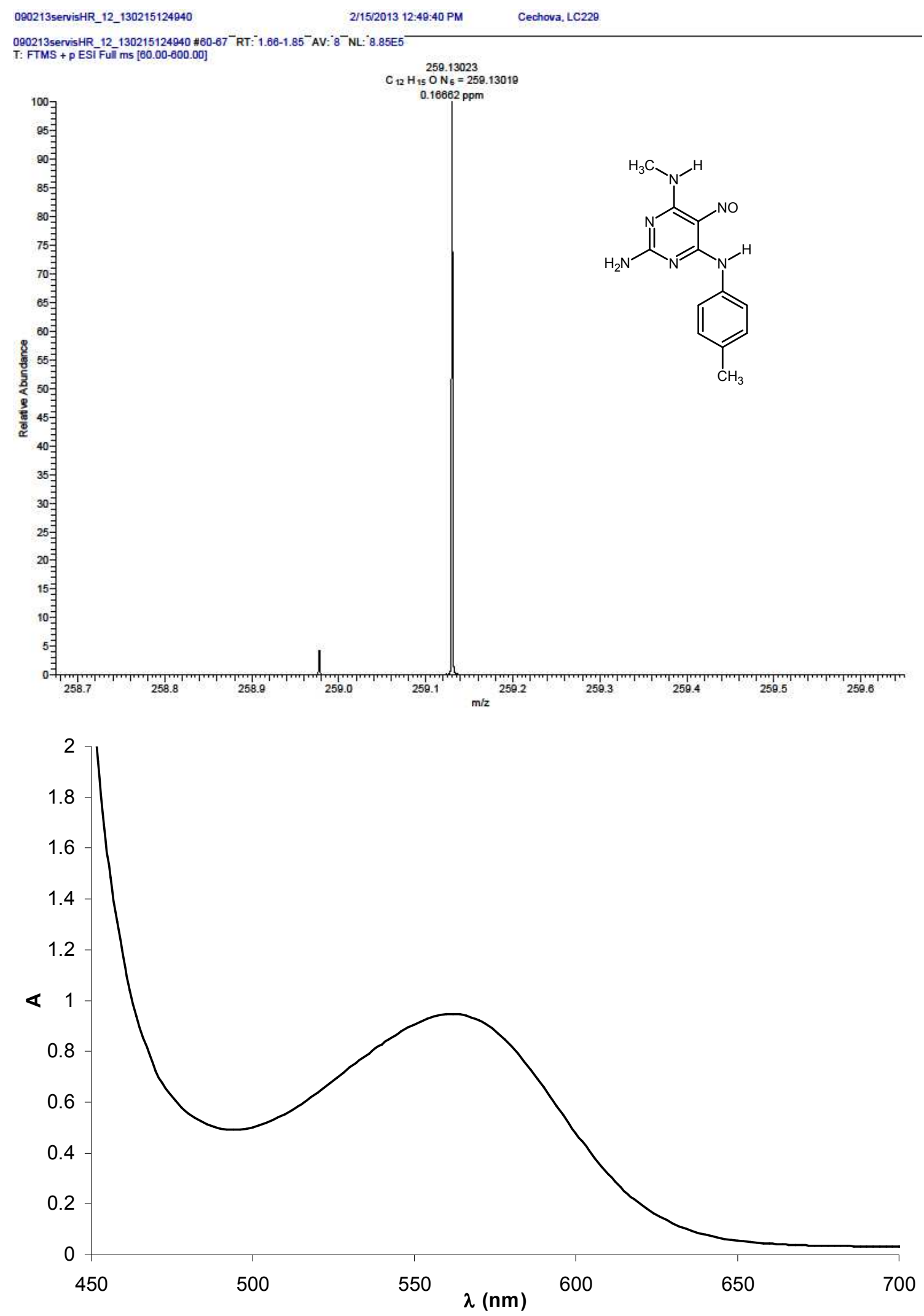

Figure S34. The HR-MS spectrum (top) and electronic spectrum (bottom) of compound $\mathbf{4 f}$. 

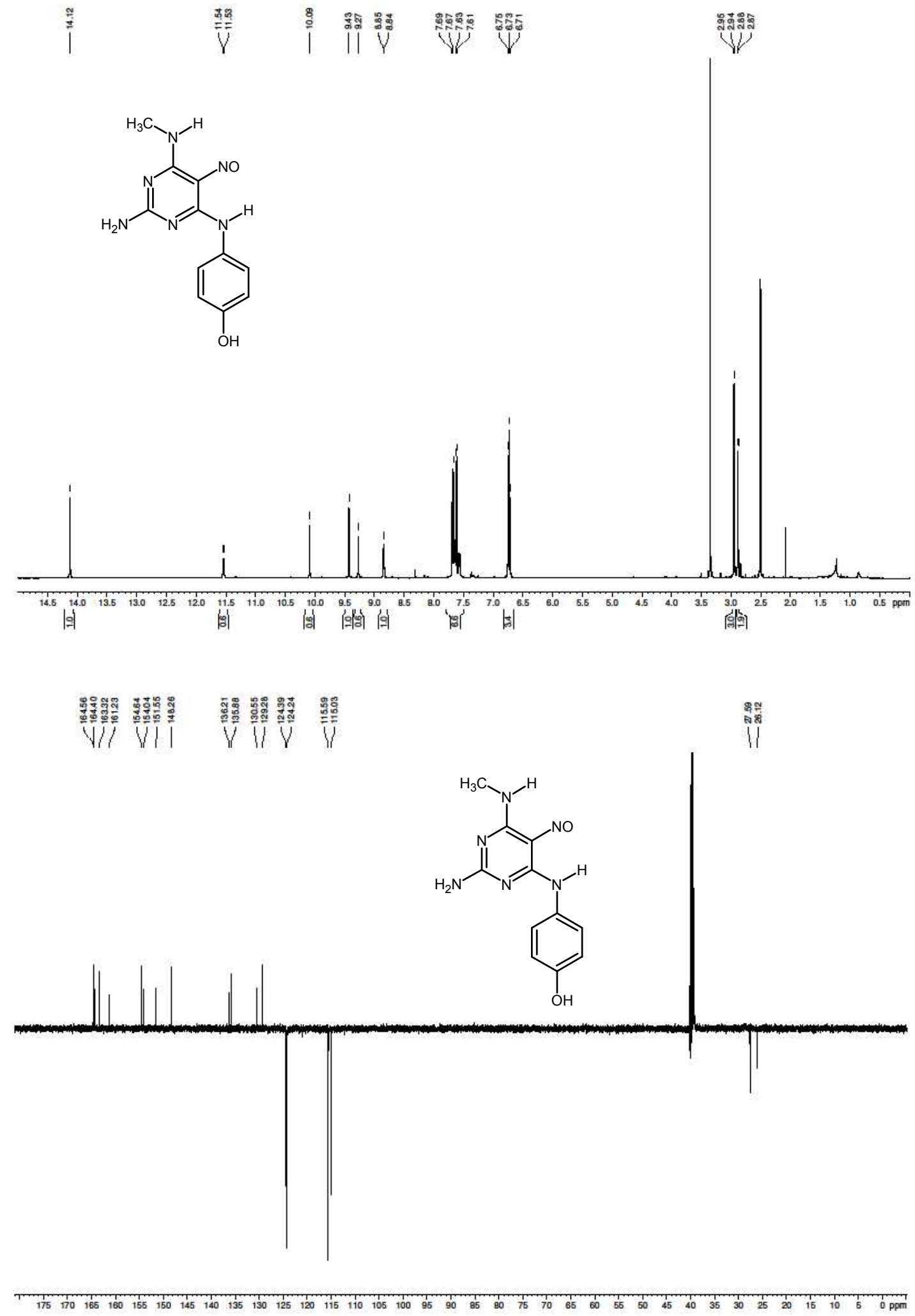

Figure S35. The NMR spectra of compound 4g. 

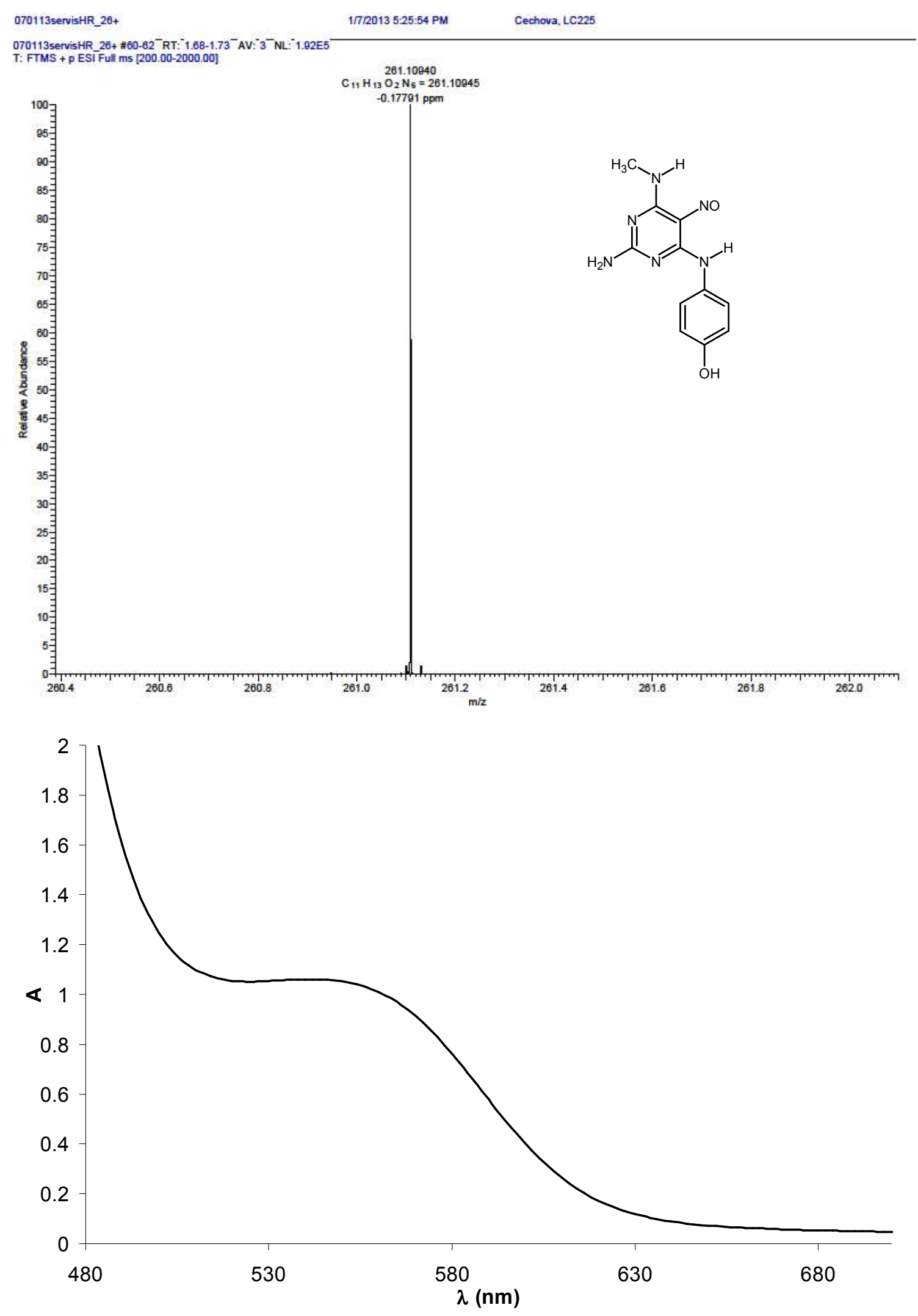

Figure S36. The HR-MS spectrum (top) and electronic spectrum (bottom) of compound $\mathbf{4 g}$. 

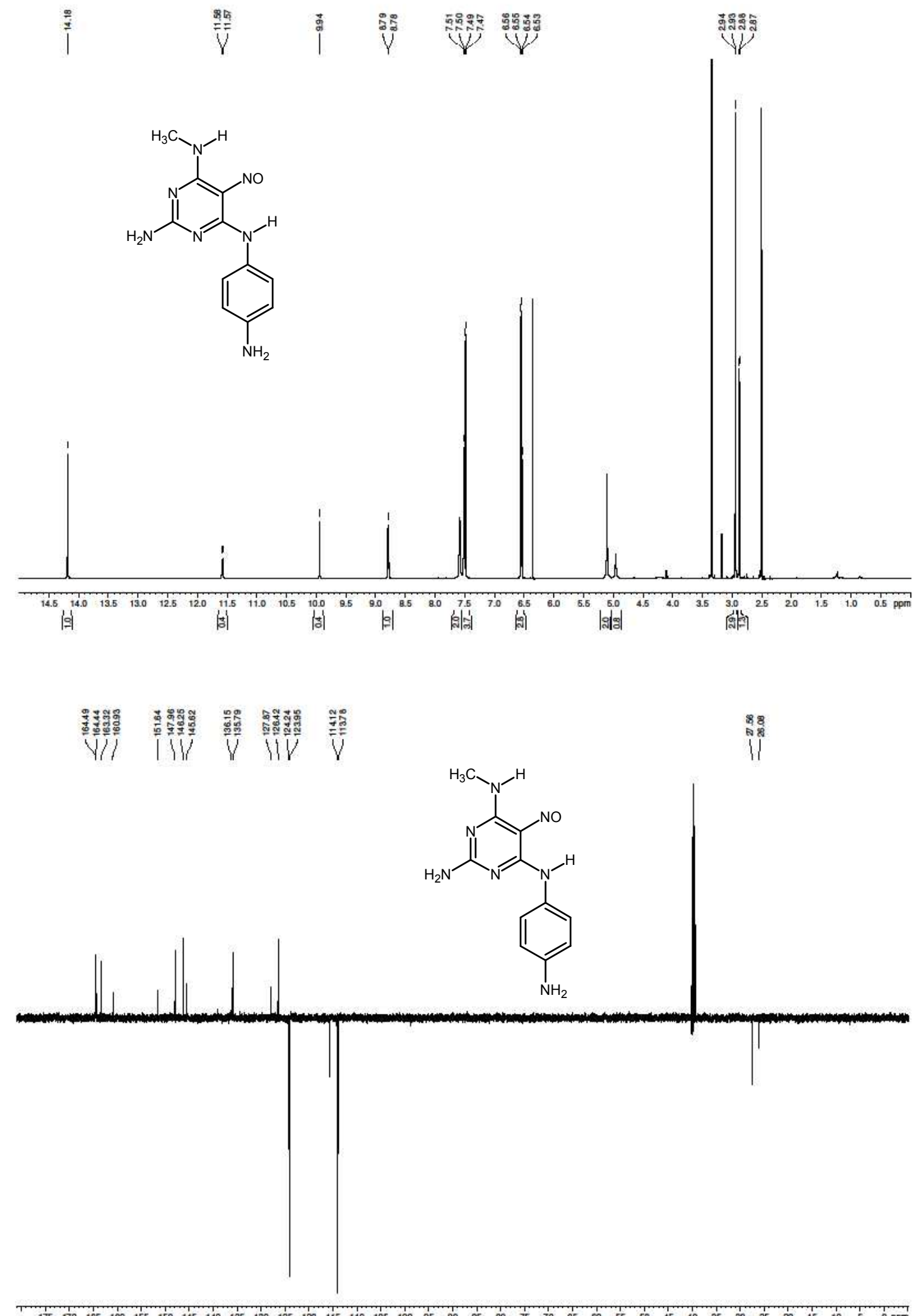

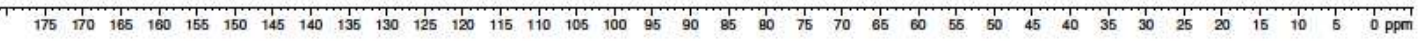

Figure S37. The NMR spectra of compound $\mathbf{4 h}$. 


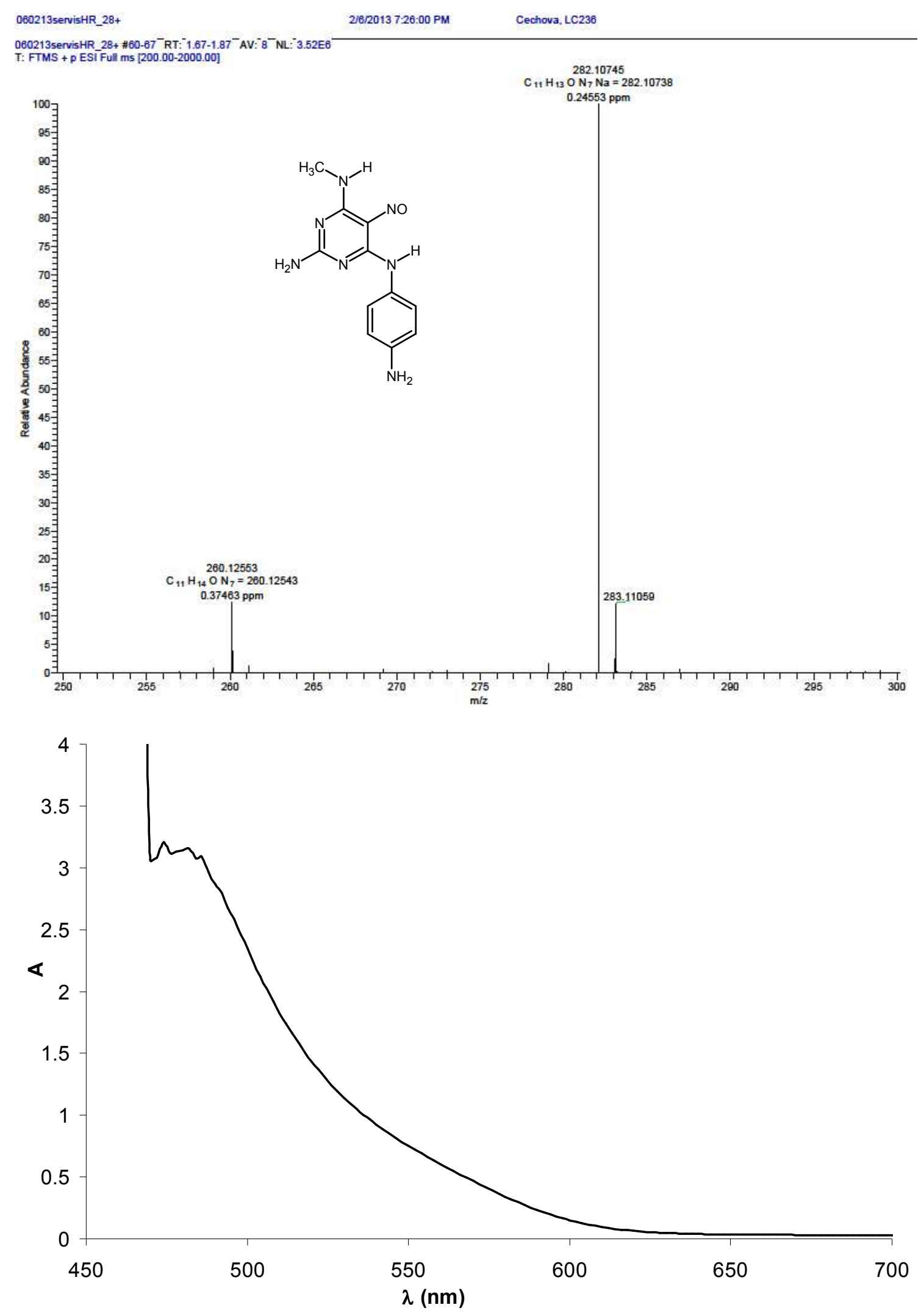

Figure S38. The HR-MS spectrum (top) and electronic spectrum (bottom) of compound $\mathbf{4 h}$. 

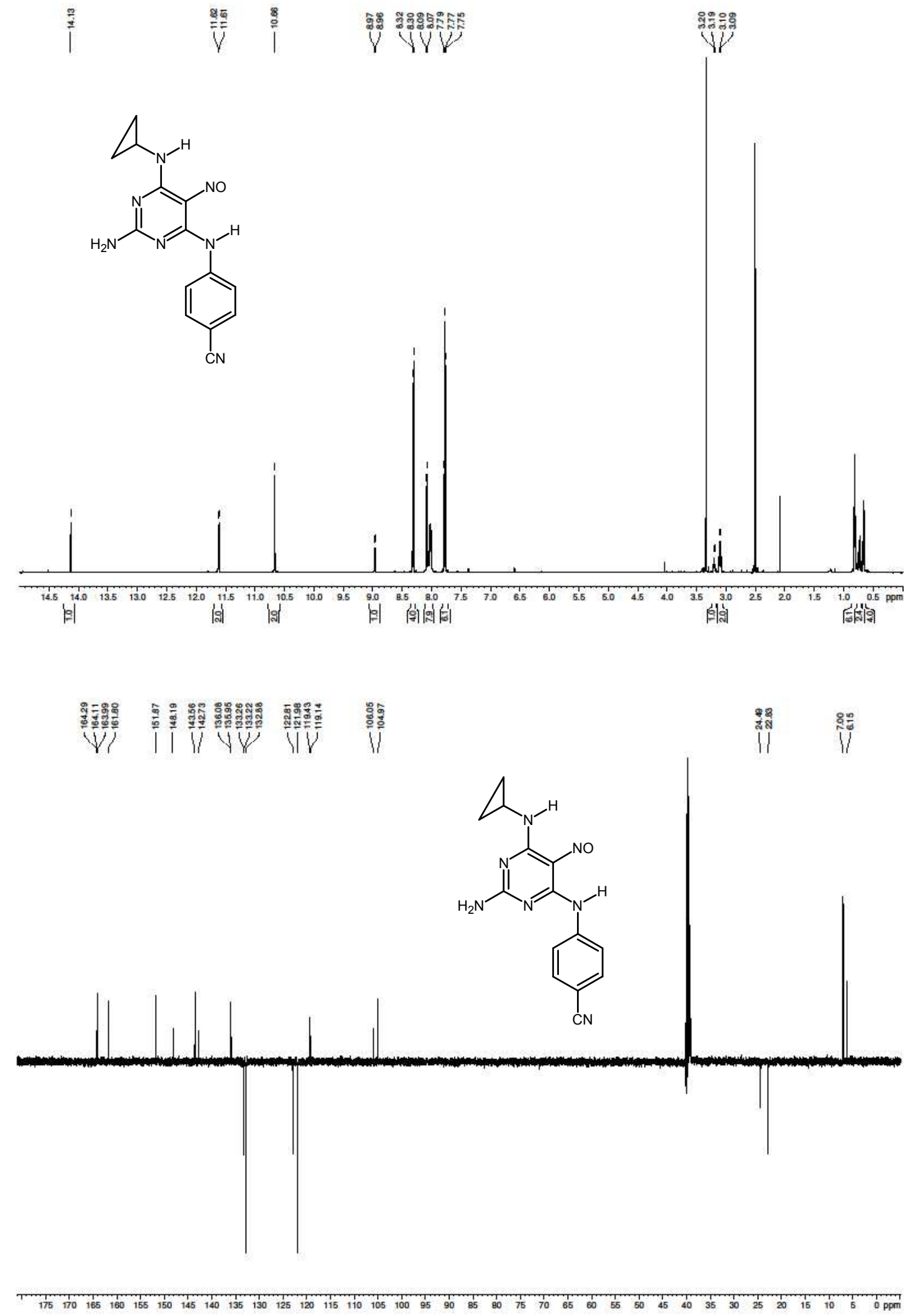

Figure S39. The NMR spectrum of compound 5a. 

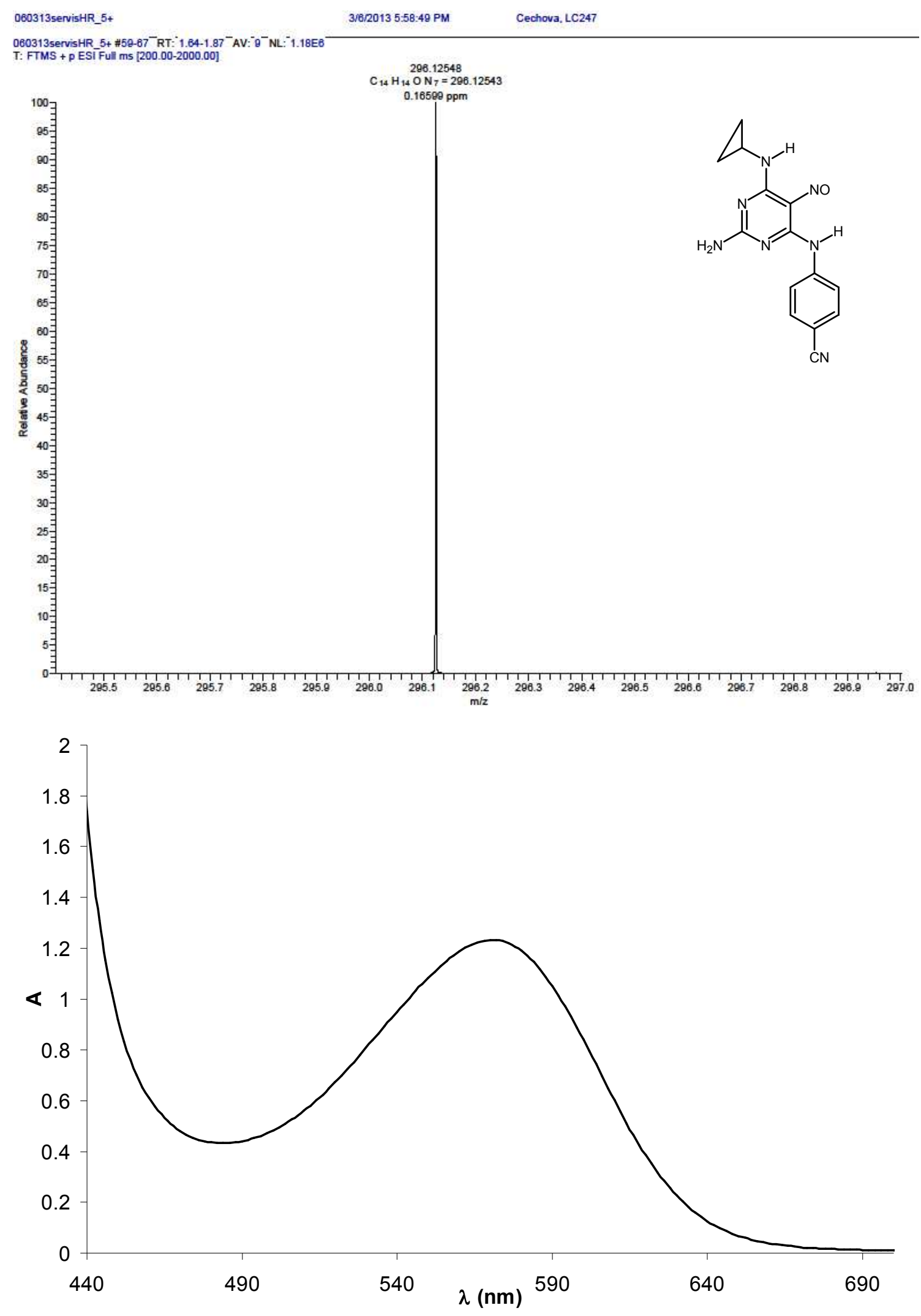

Figure S40. The HR-MS spectrum (top) and electronic spectrum (bottom) of compound 5a. 

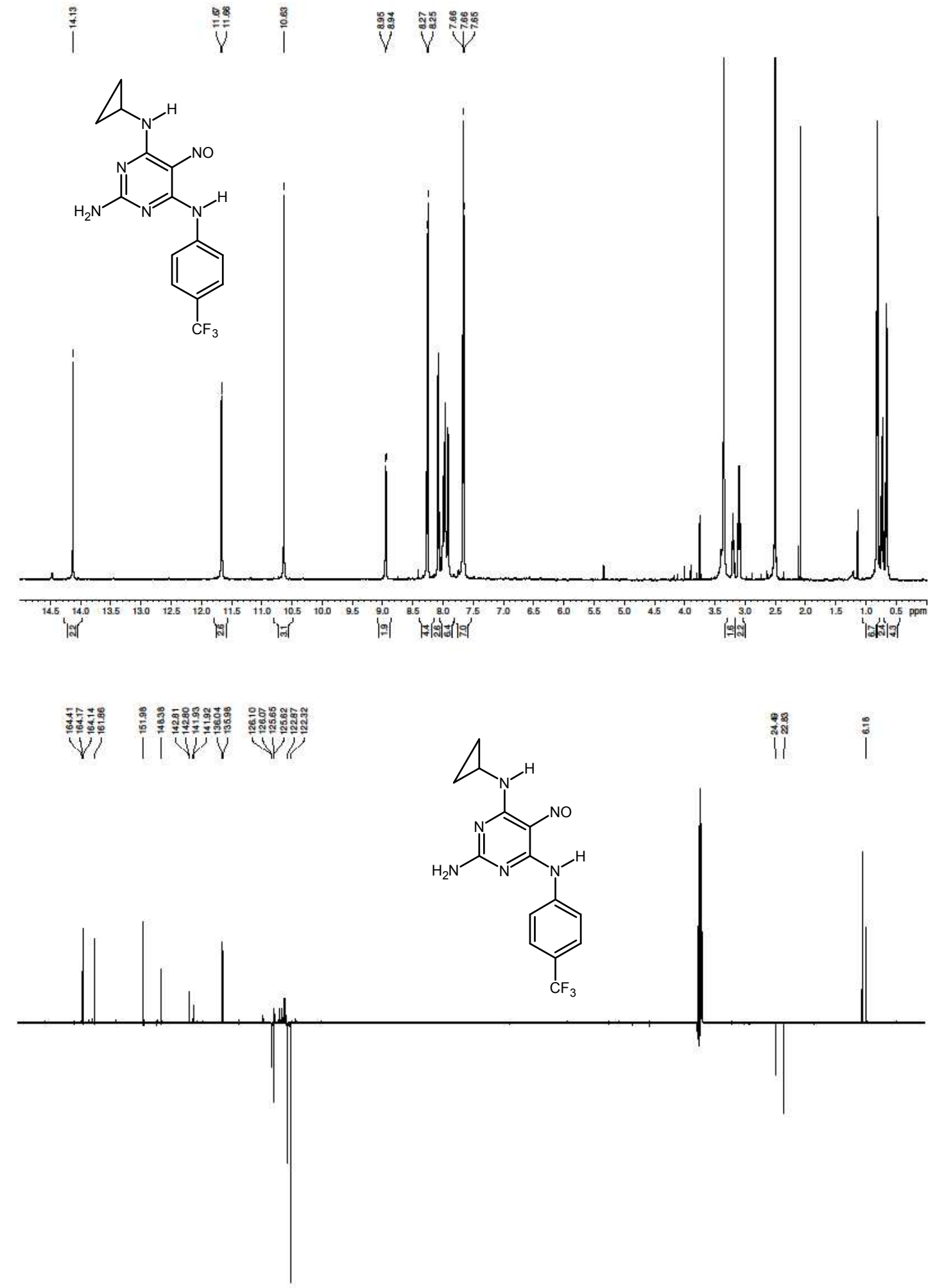

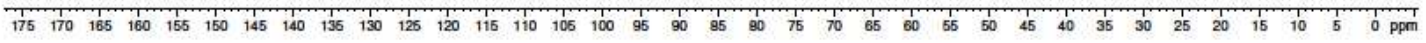

Figure S41. The NMR spectra of compound $\mathbf{5 b}$. 

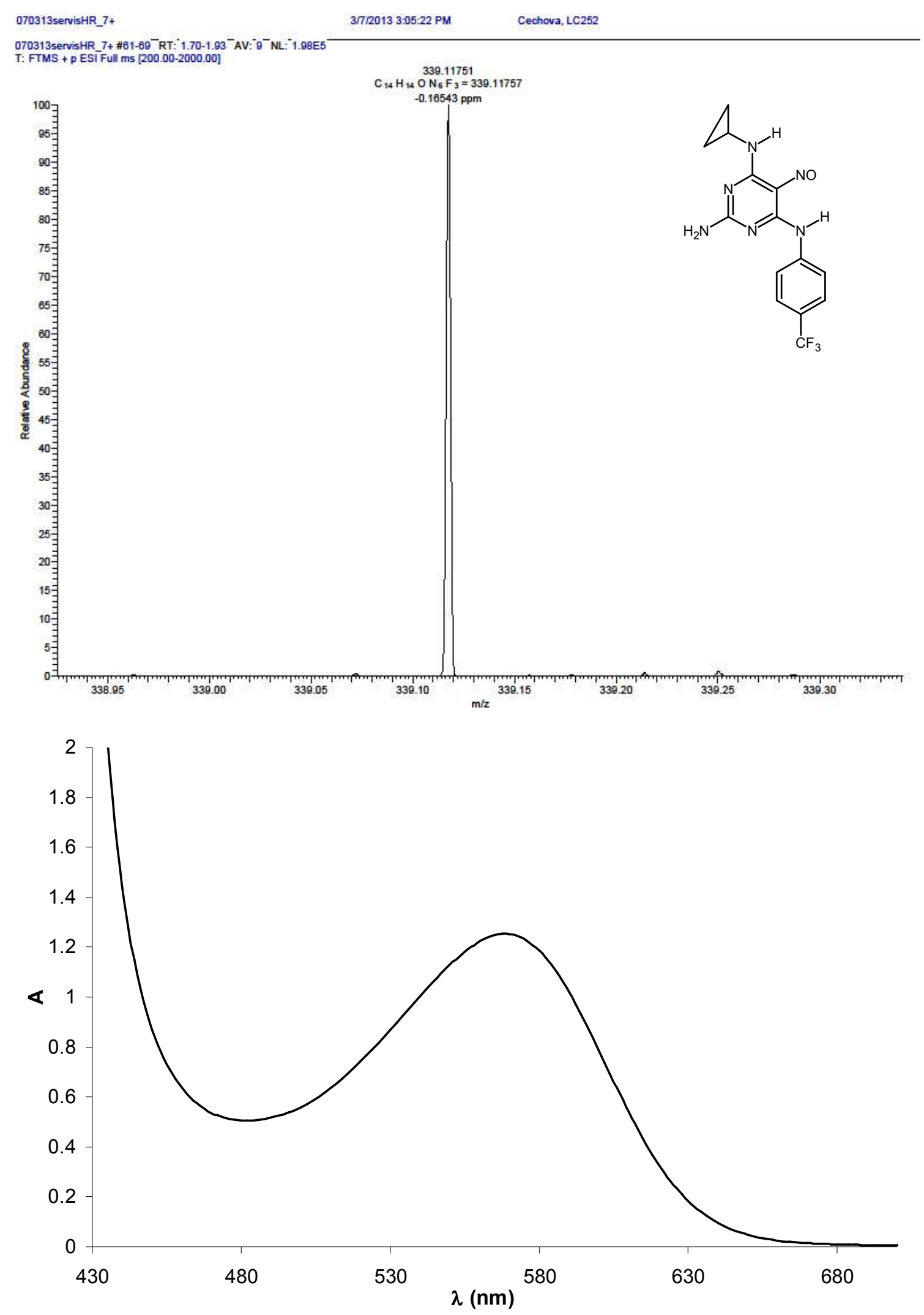

Figure S42. The HR-MS spectrum (top) and electronic spectrum (bottom) of compound $\mathbf{5 b}$. 
i

$\frac{88}{\sqrt{2}}$

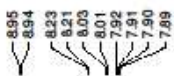

(c)

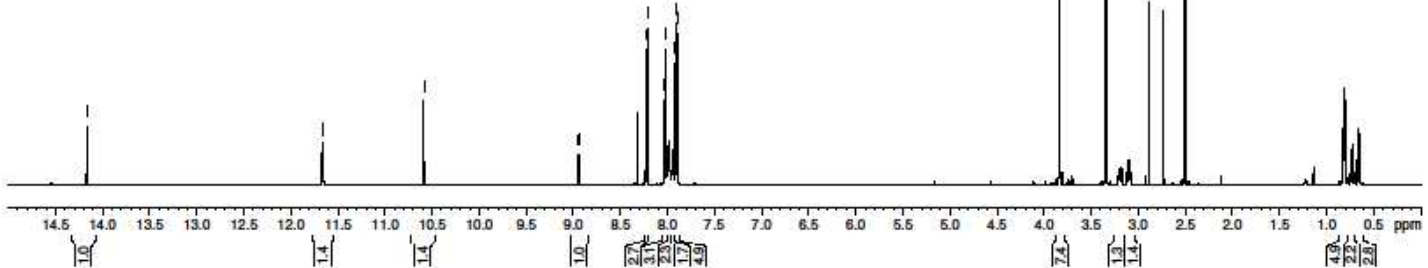

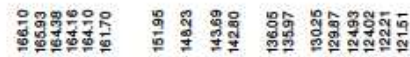

WW' II V V V YII

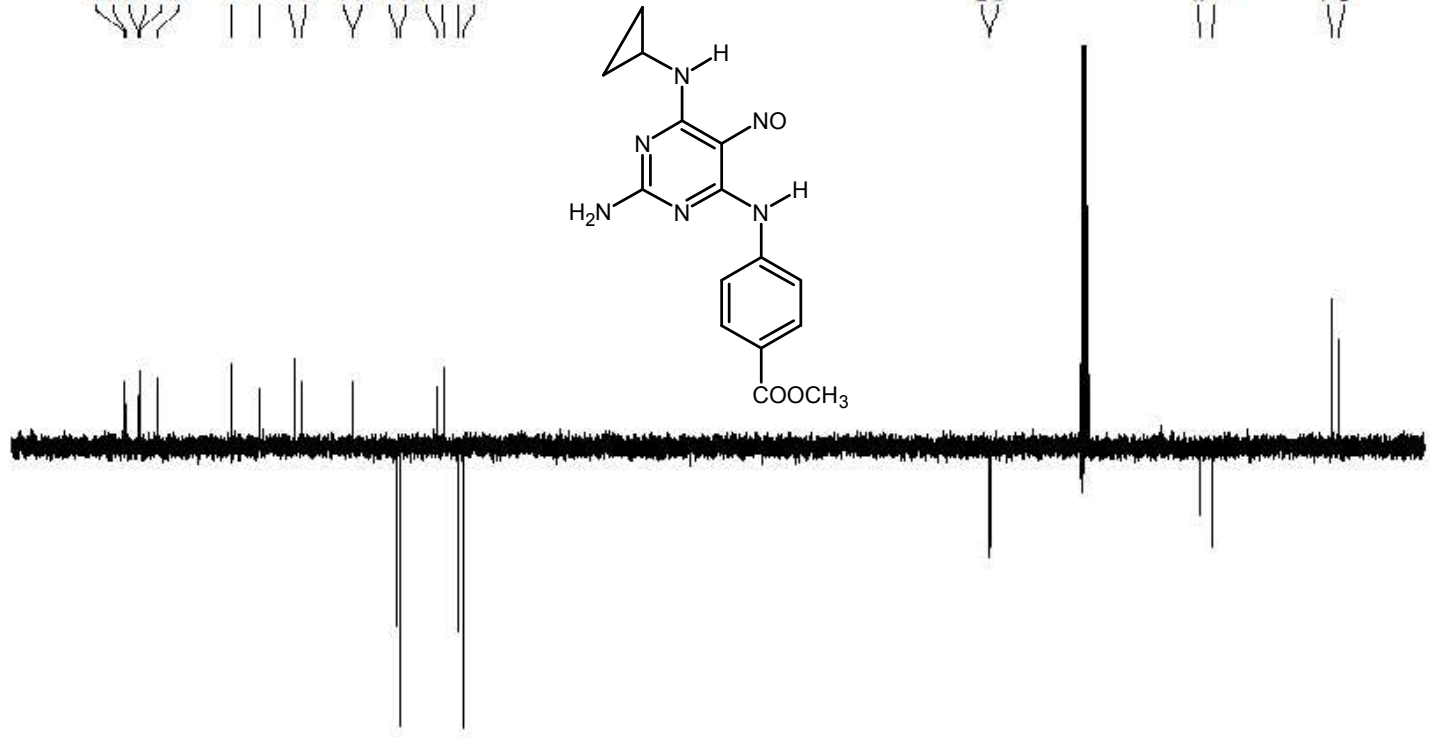

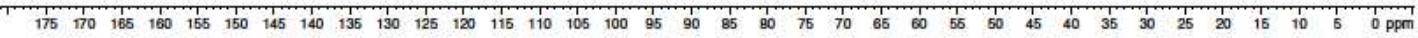

Figure S43. The NMR spectra of compound $\mathbf{5 c}$. 

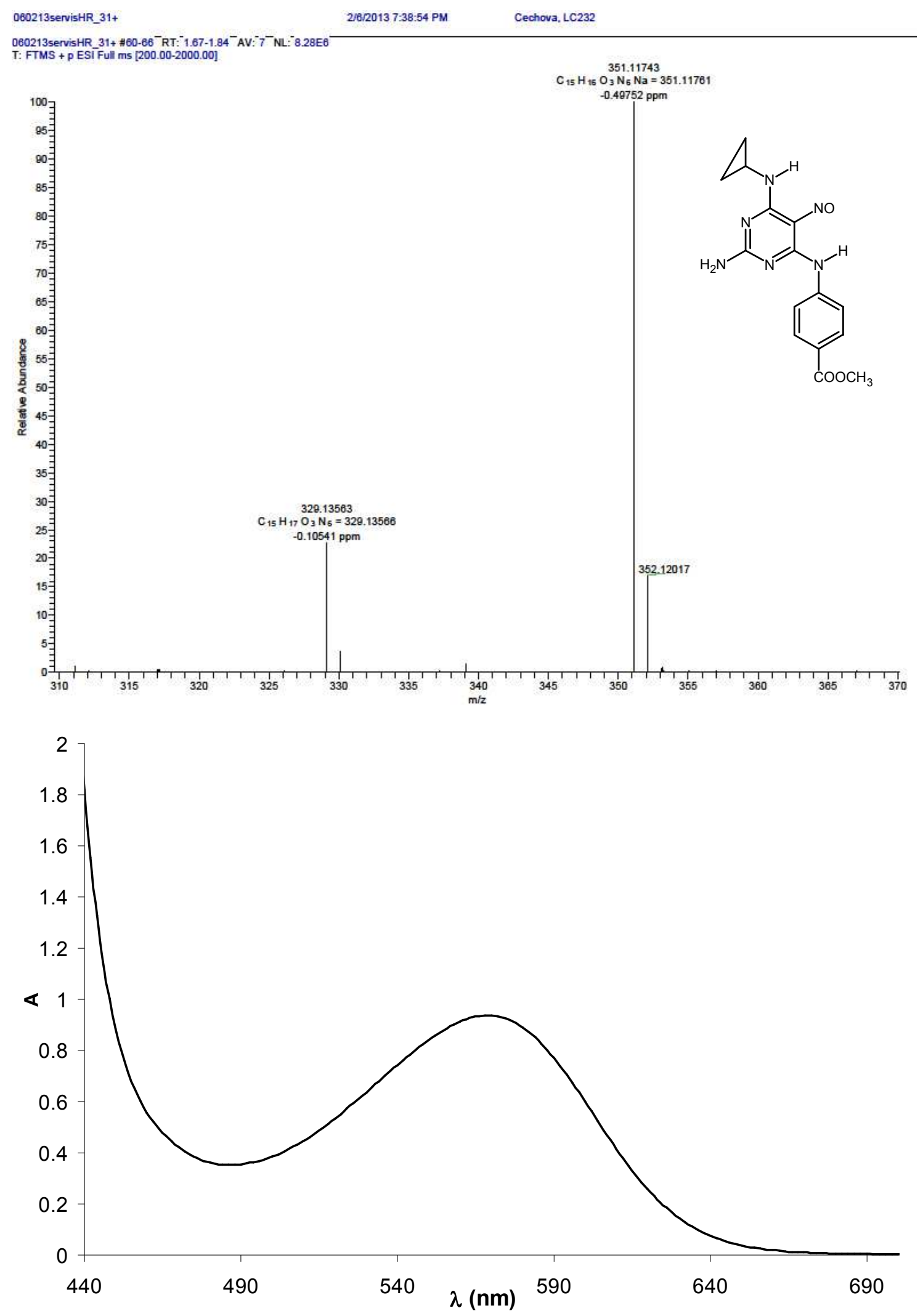

Figure S44. The HR-MS spectrum (top) and electronic spectrum (bottom) of compound 5c. 

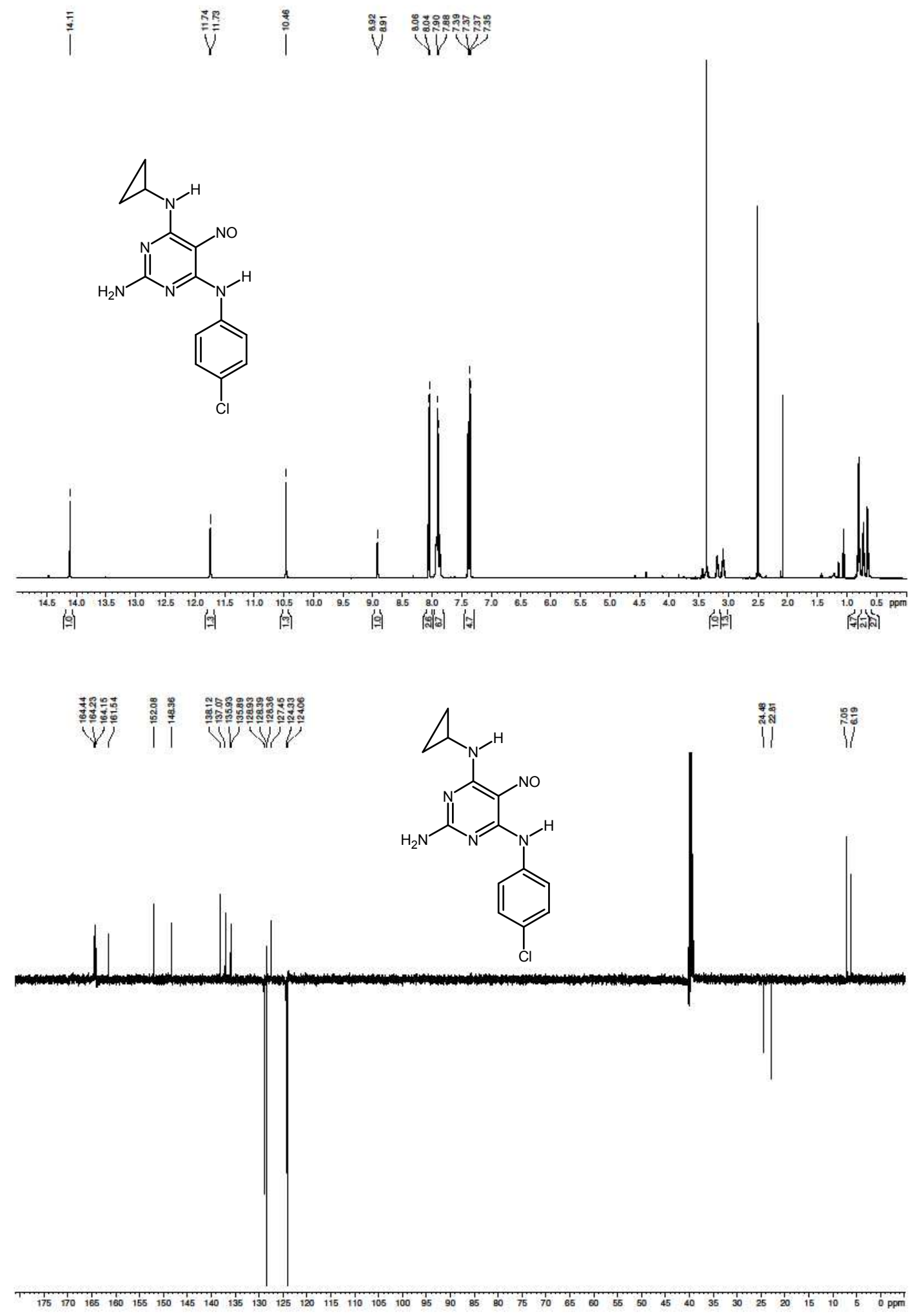

Figure S45. The NMR spectra of the compound $\mathbf{5 d}$. 


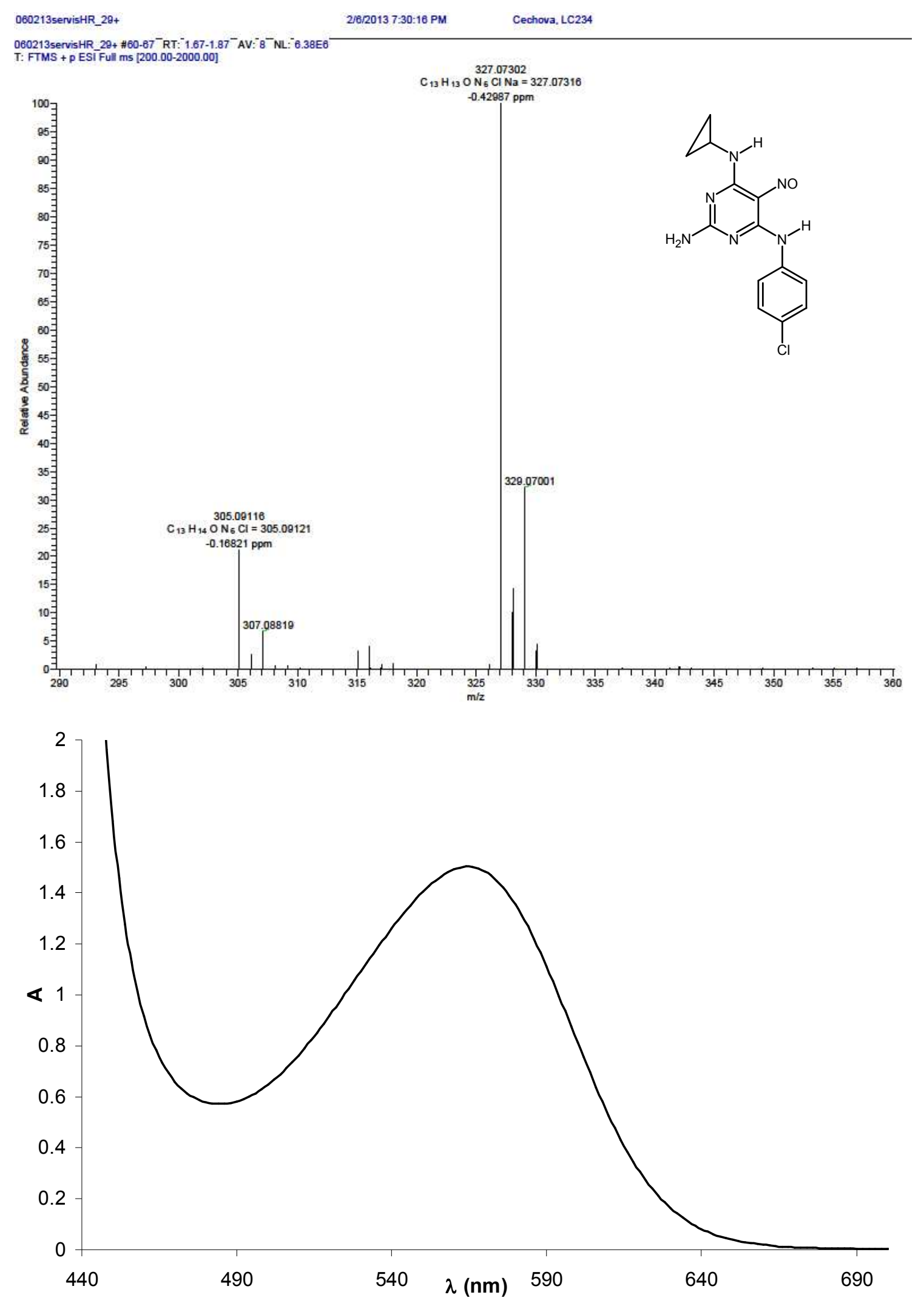

Figure S46. The HR-MS spectrum (top) and electronic spectrum (bottom) of compound 5d. 

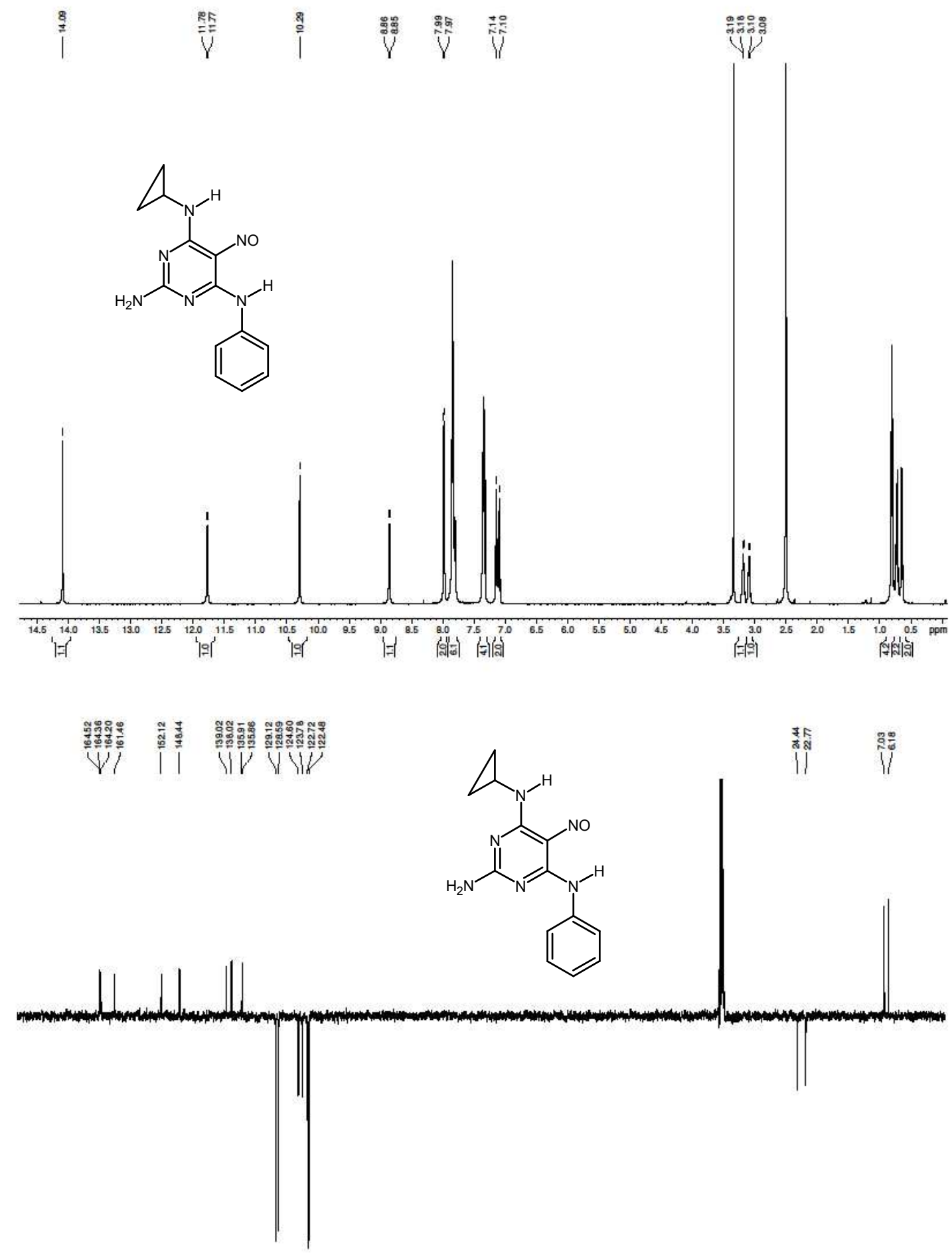

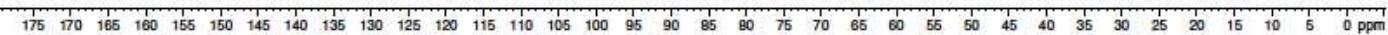

Figure S47. The NMR spectra of the compound $5 \mathbf{e}$. 

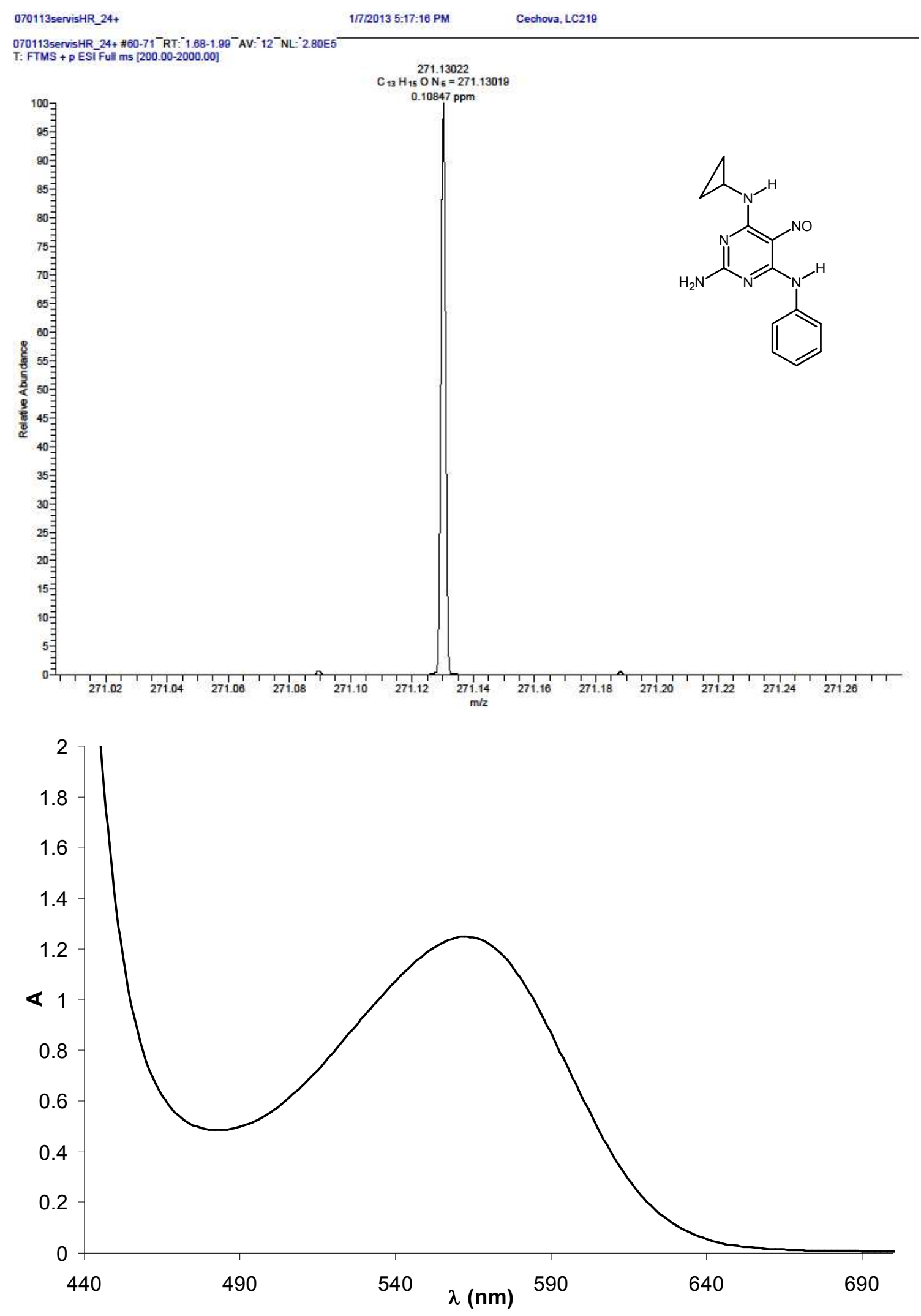

Figure S48. The HR-MS spectrum (top) and electronic spectrum (bottom) of compound $5 \mathbf{e}$. 

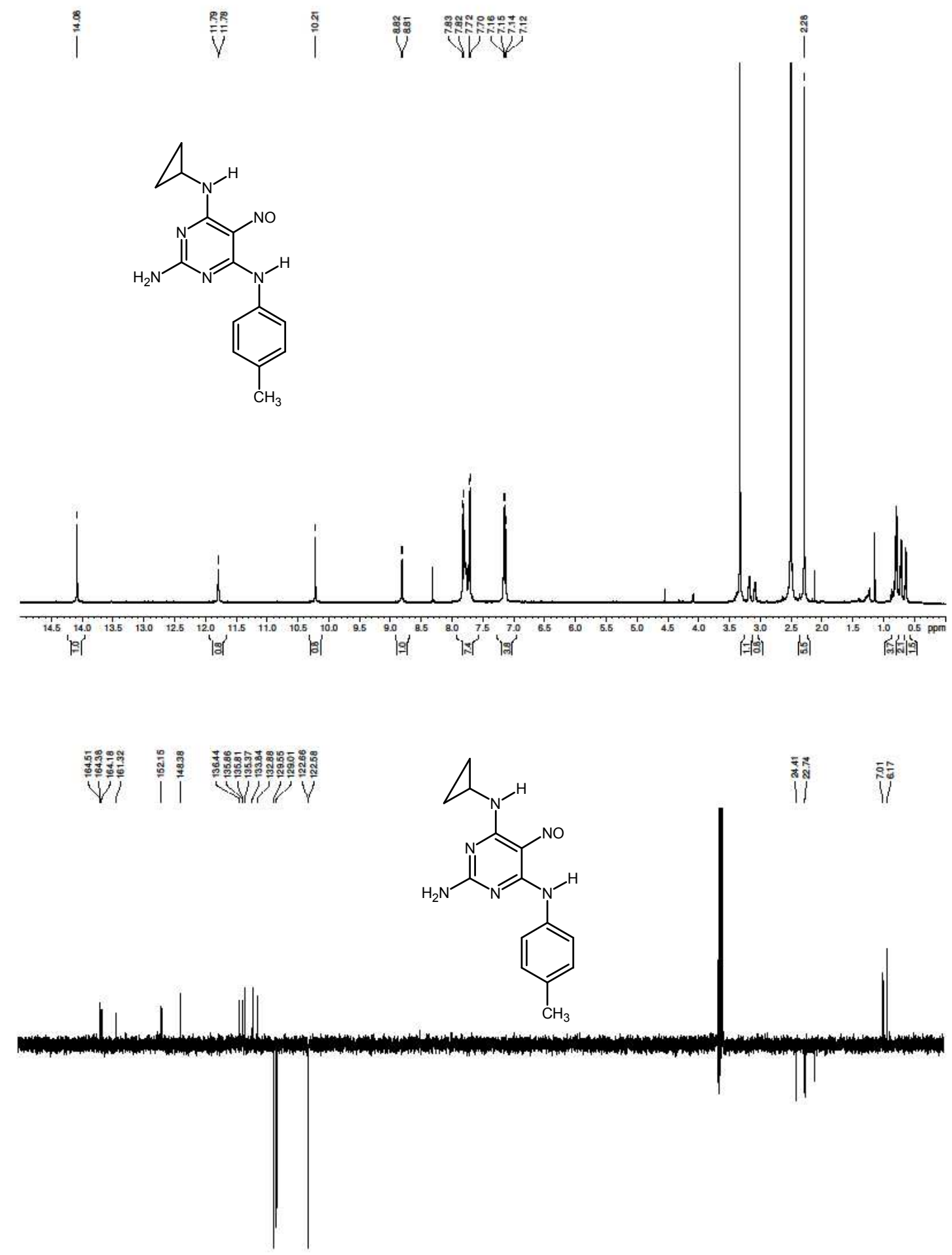

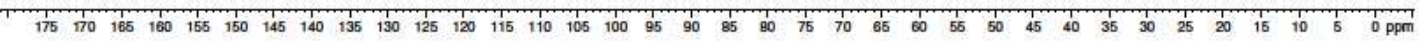

Figure S49. The NMR spectra of the compound $\mathbf{5 f}$. 

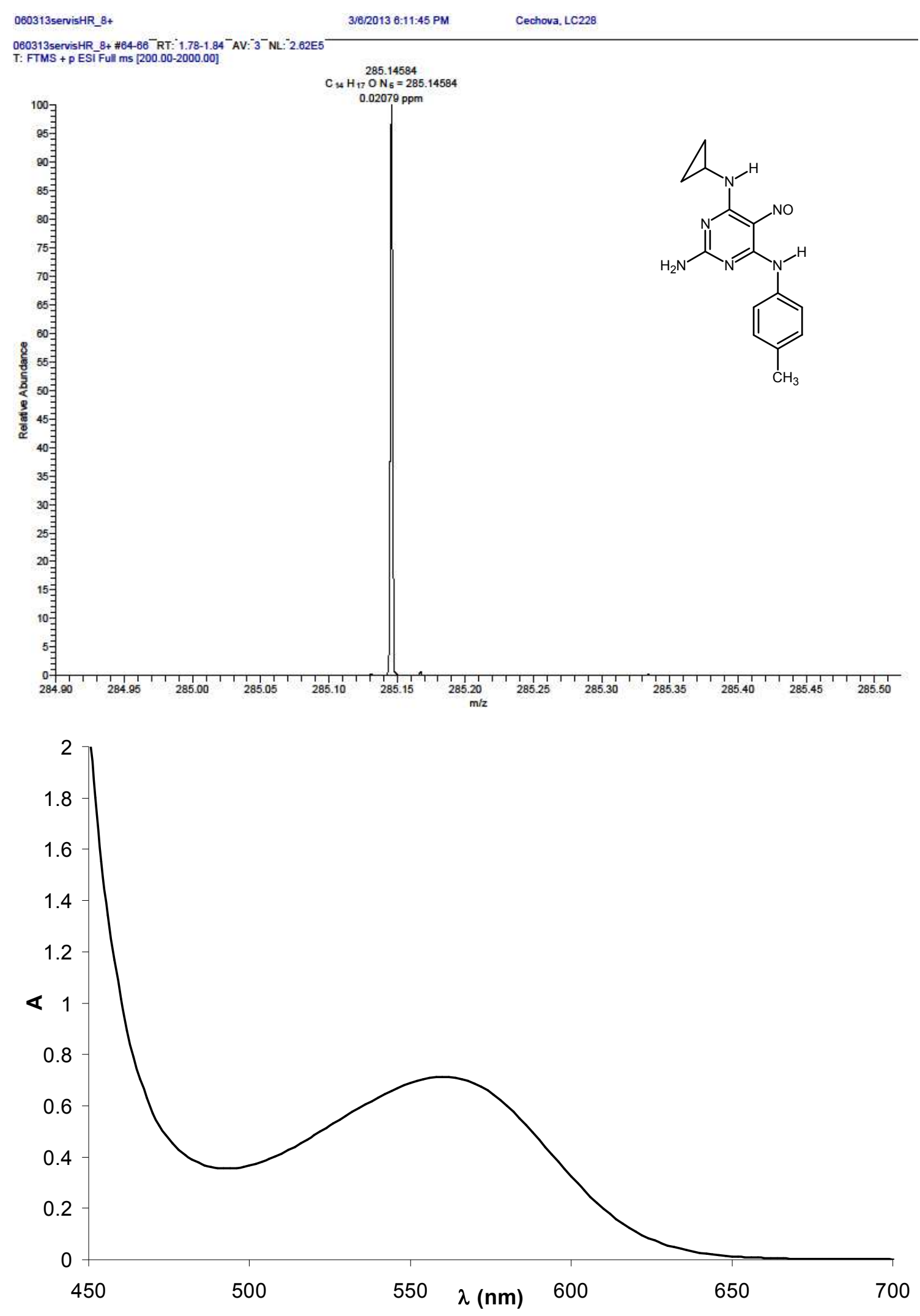

Figure S50. The HR-MS spectrum (top) and electronic spectrum (bottom) of compound $\mathbf{5 f}$. 

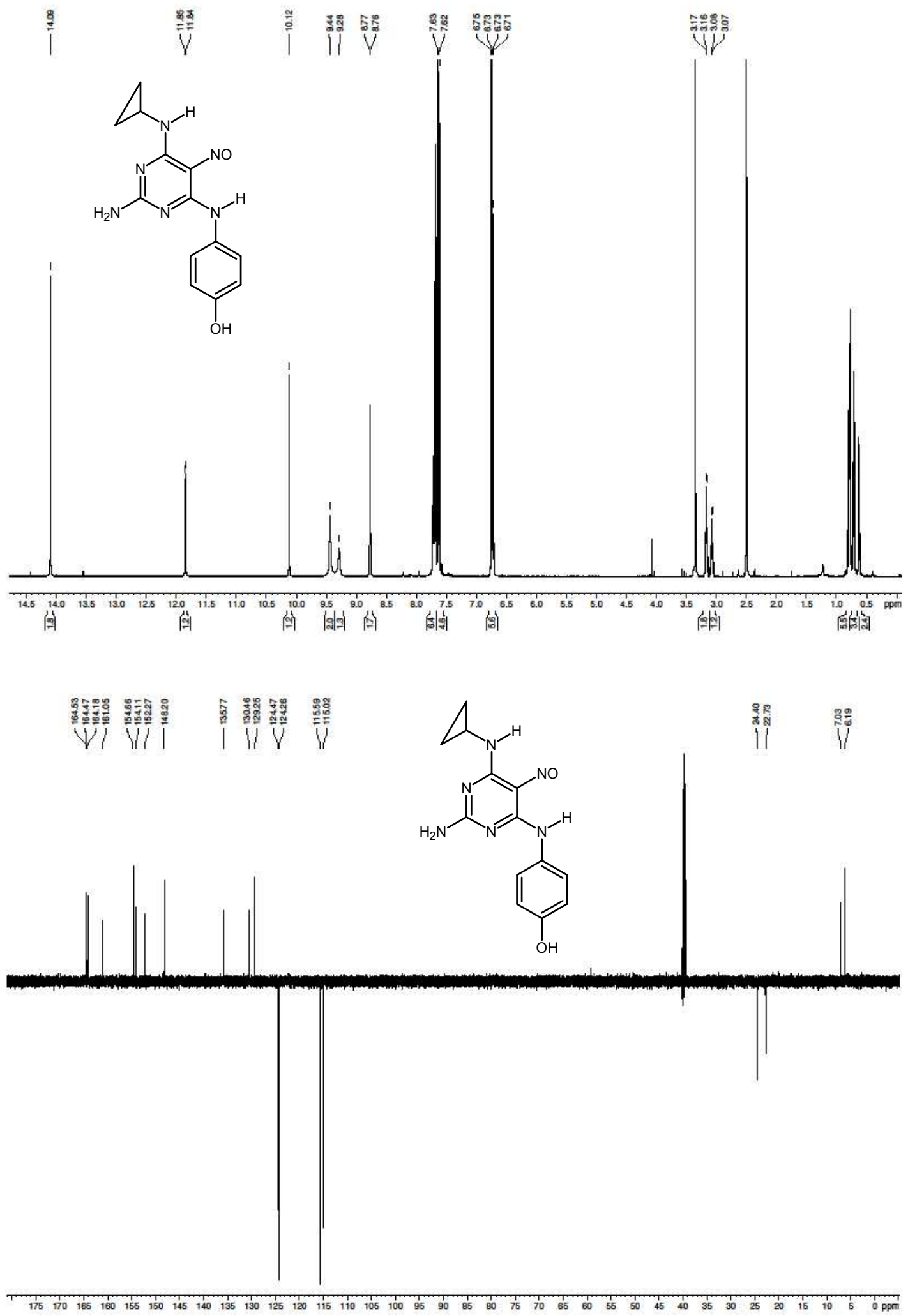

Figure S51. The NMR spectra of the compound 5g. 

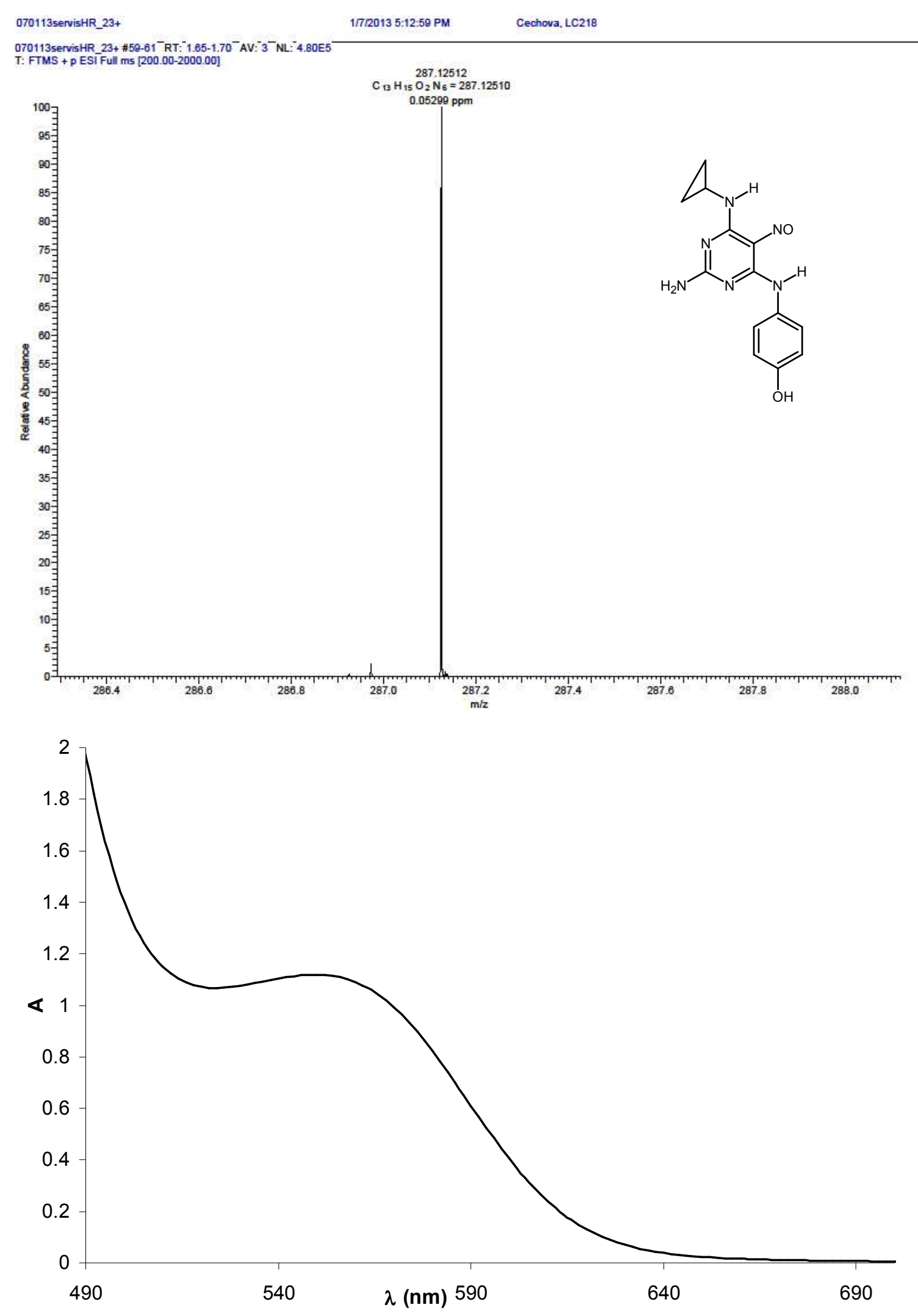

Figure S52. The HR-MS spectrum (top) and electronic spectrum (bottom) of compound 5g. 


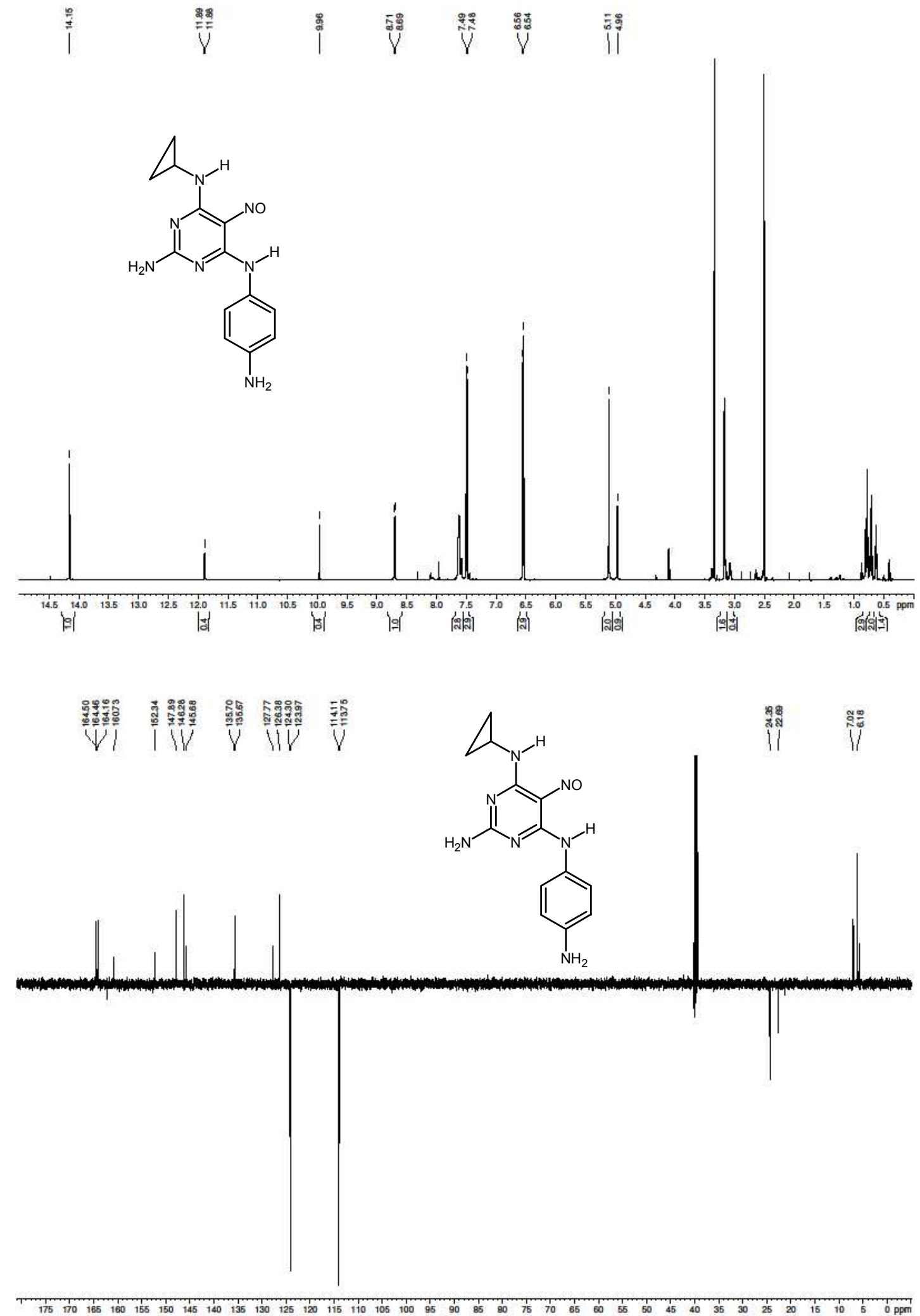

Figure S53. The NMR spectra of the compound $\mathbf{5 h}$. 

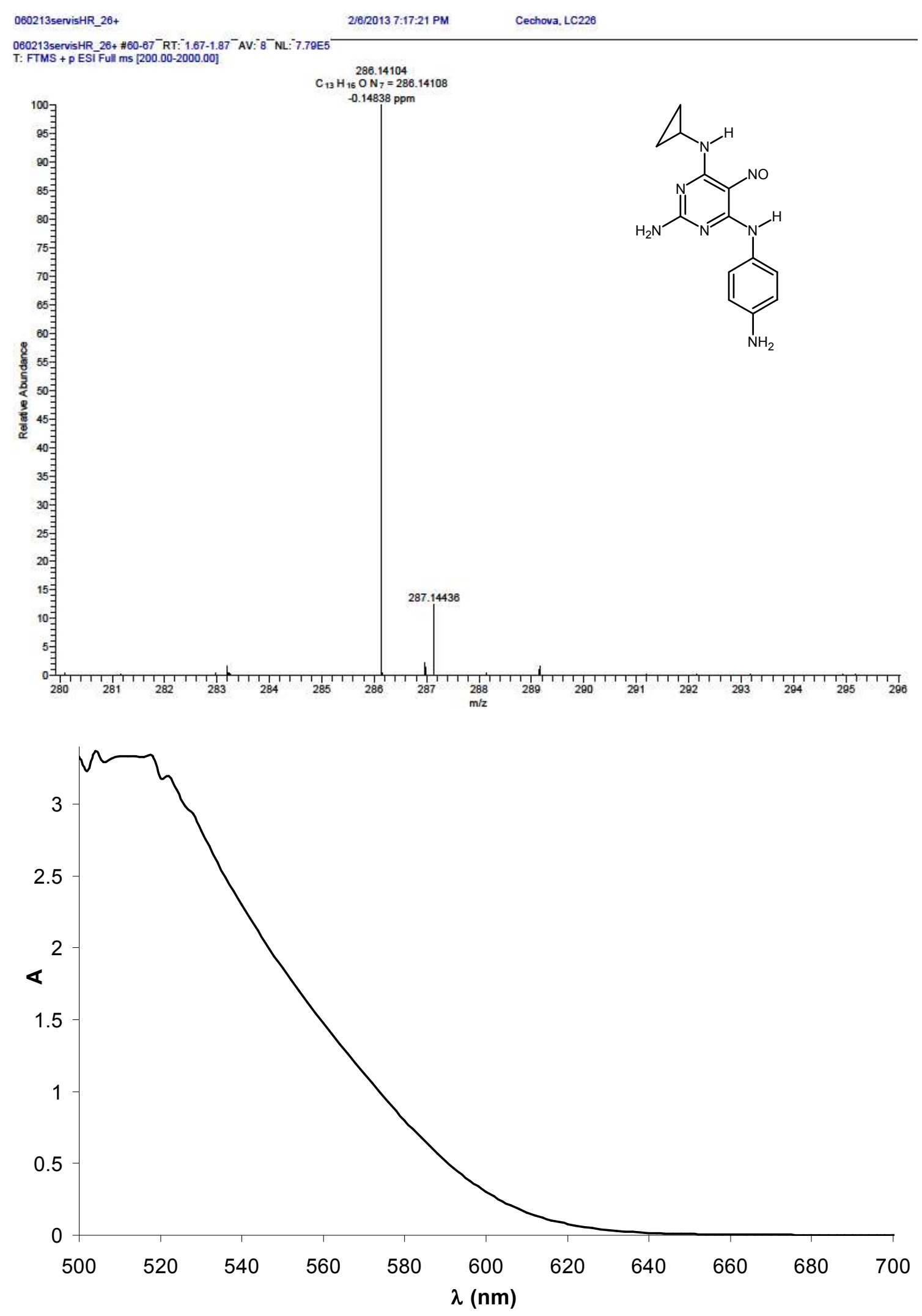

Figure S54. The HR-MS spectrum (top) and electronic spectrum (bottom) of compound $\mathbf{5 h}$. 

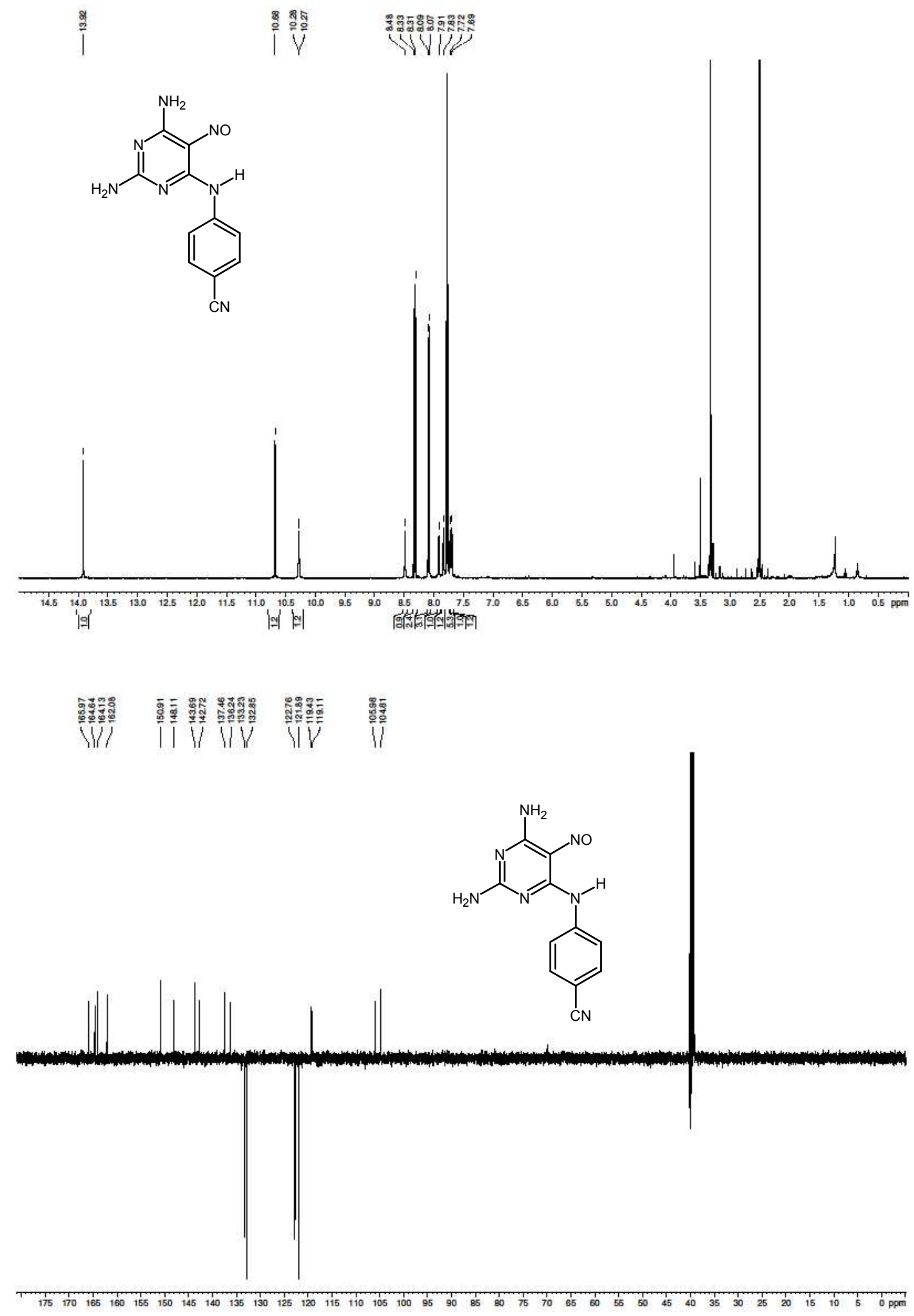

Figure S55. The NMR spectra of compound 6a. 

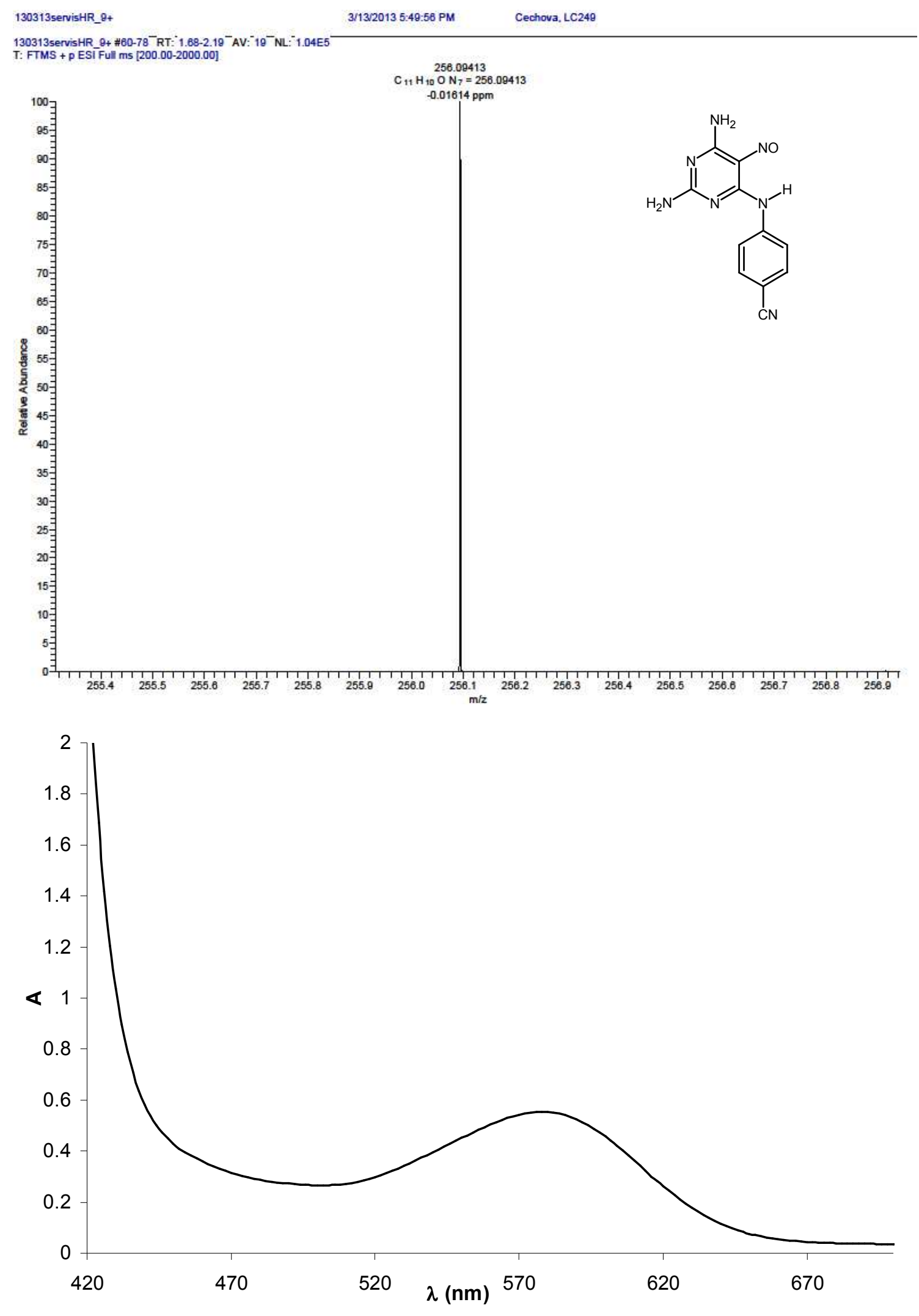

Figure S56. The HR-MS spectrum (top) and electronic spectrum (bottom) of compound 6a. 
$i^{\frac{9}{2}}$

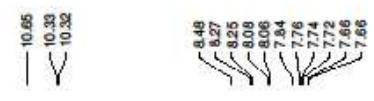<smiles>Nc1nc(N)c([N+](=O)[O-])c(Nc2ccc(C(F)(F)F)cc2)n1</smiles>
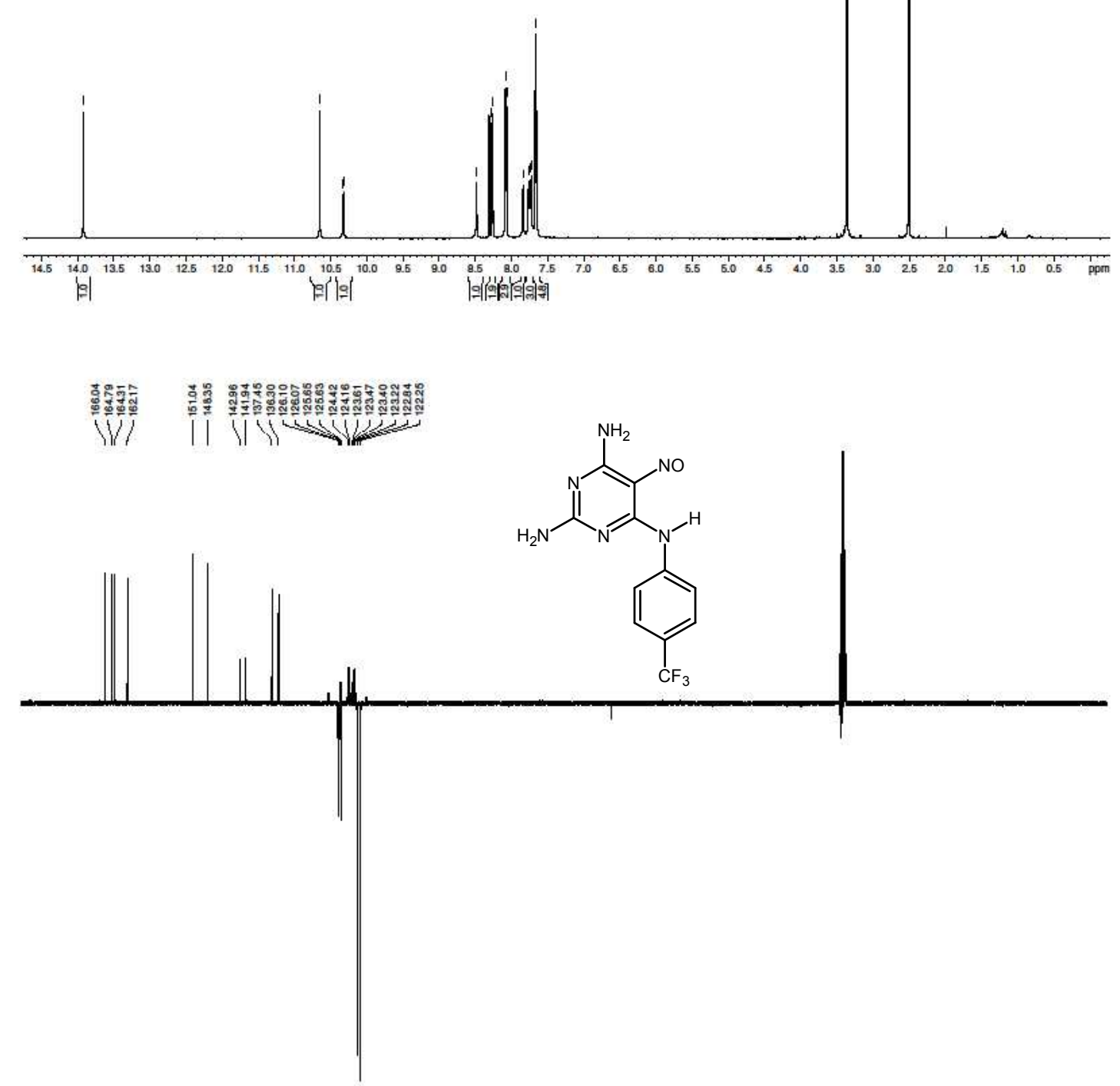

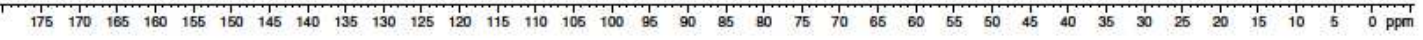

Figure S57. The NMR spectra of compound $\mathbf{6 b}$. 

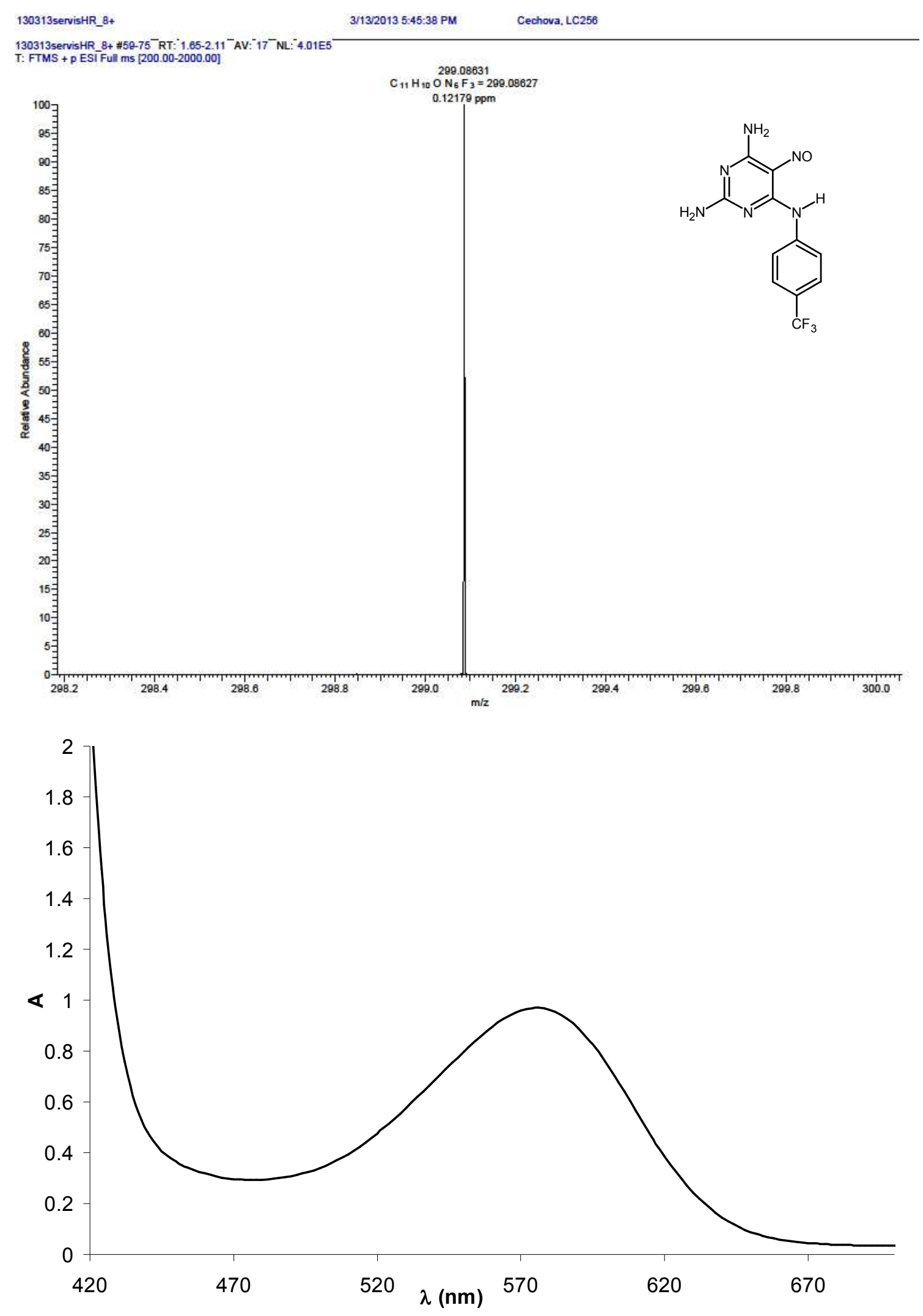

Figure S58. The HR-MS spectrum (top) and electronic spectrum (bottom) of compound $\mathbf{6 b}$. 

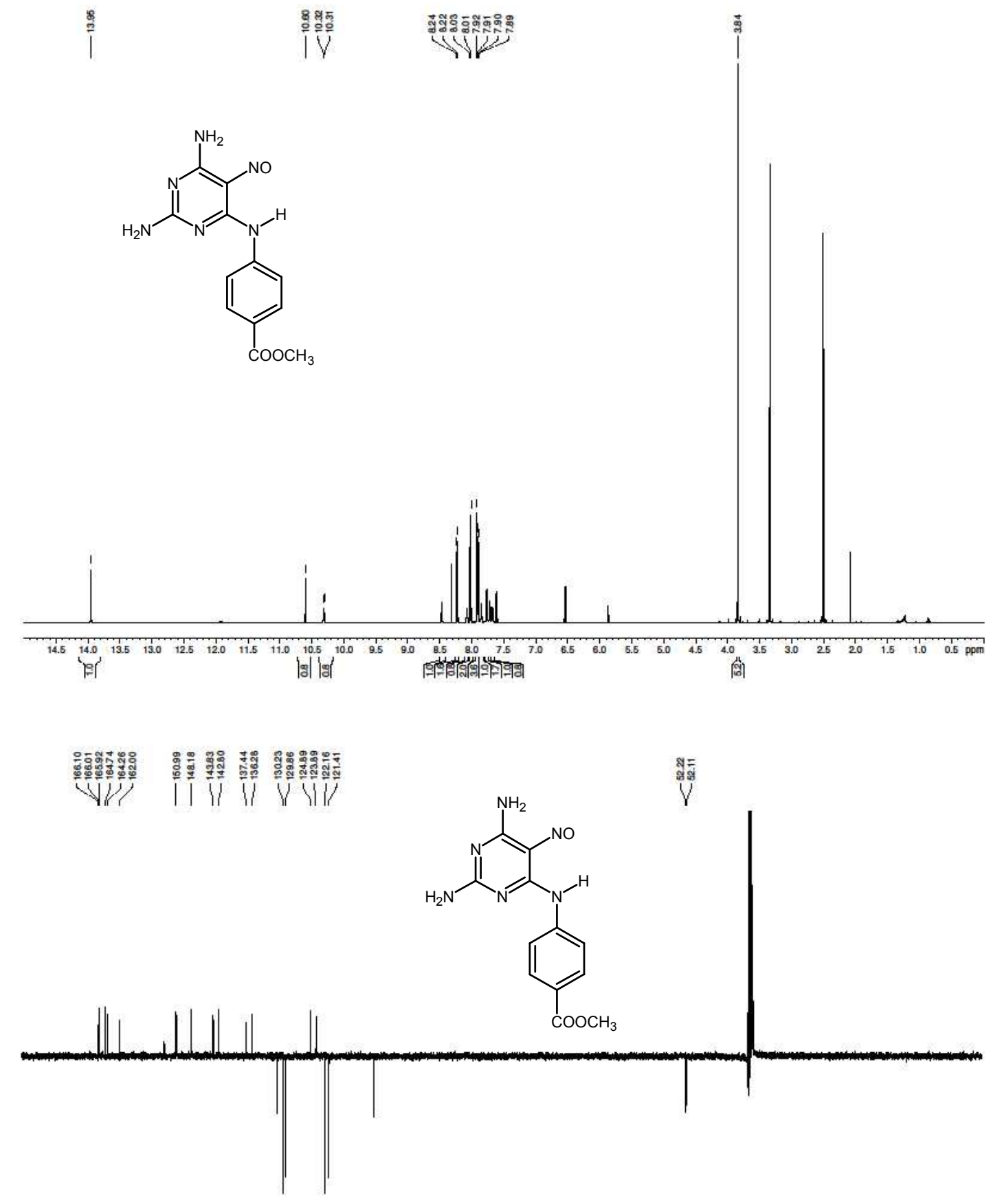

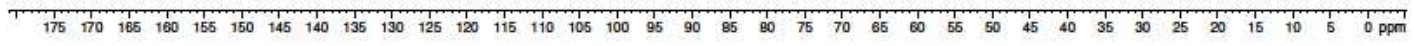

Figure S59. The NMR spectra of compound $6 \mathbf{c}$. 

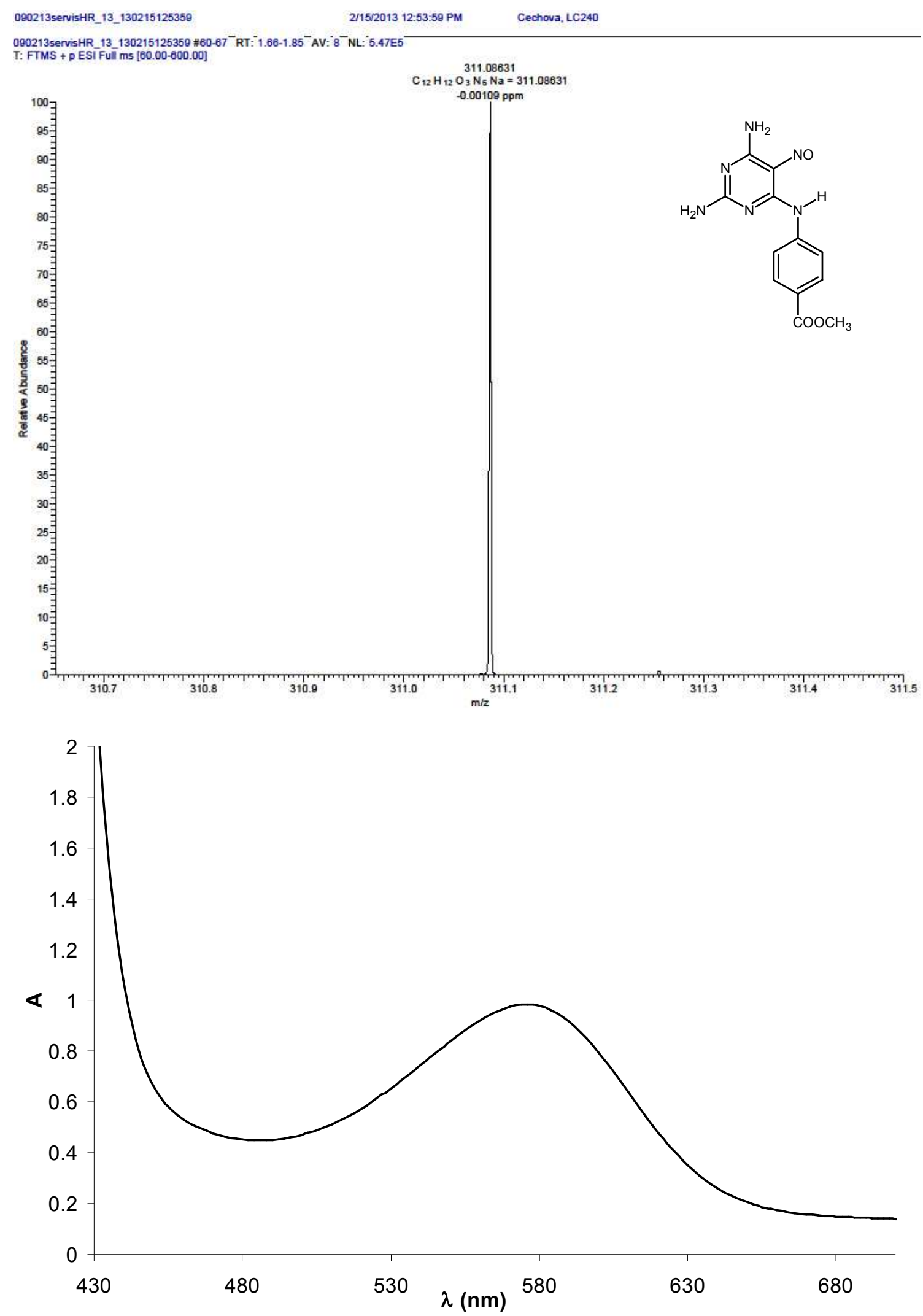

Figure S60. The HR-MS spectrum (top) and electronic spectrum (bottom) of compound $\mathbf{6 c}$. 


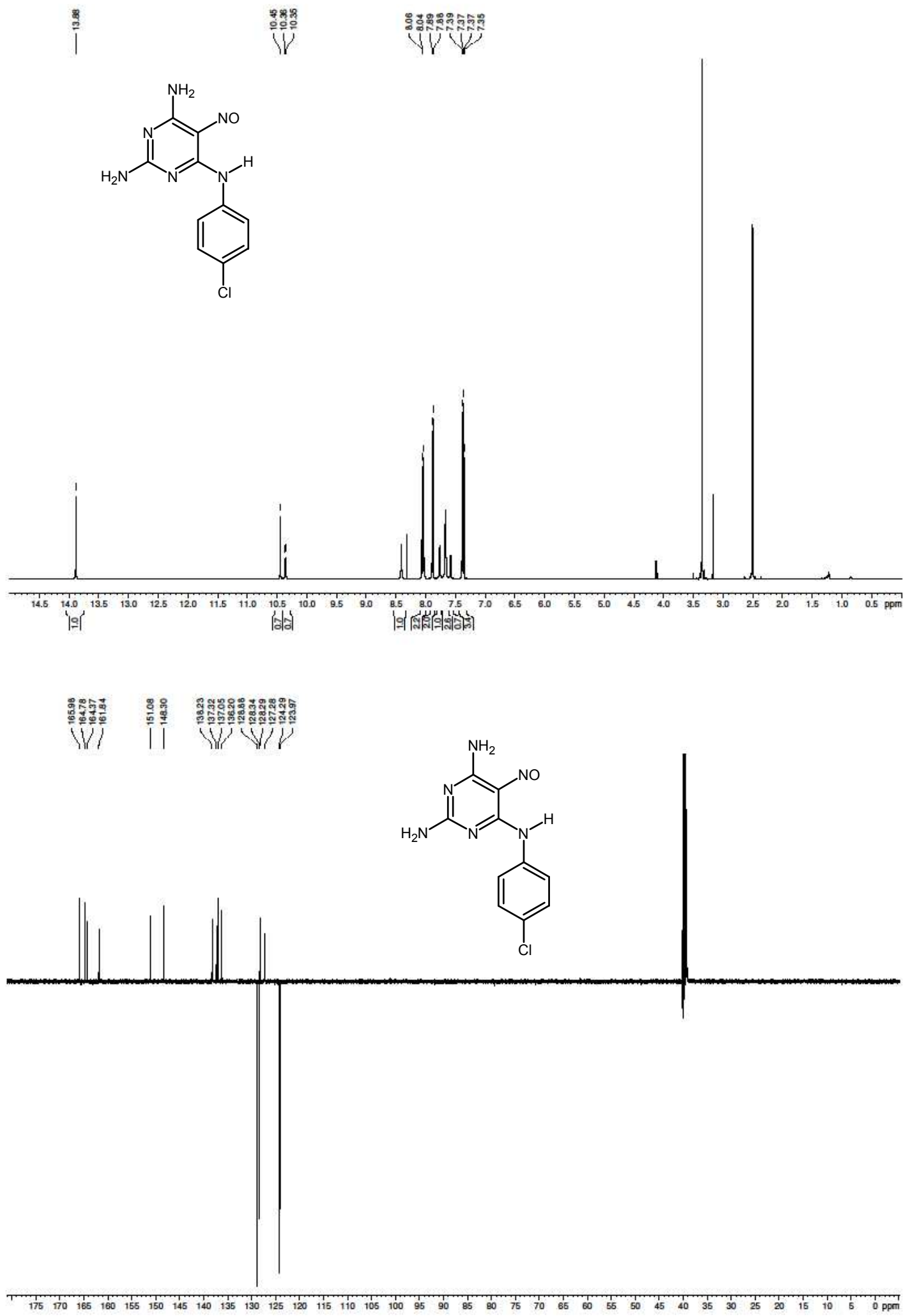

Figure S61. The NMR spectra of compound $\mathbf{6 d}$. 

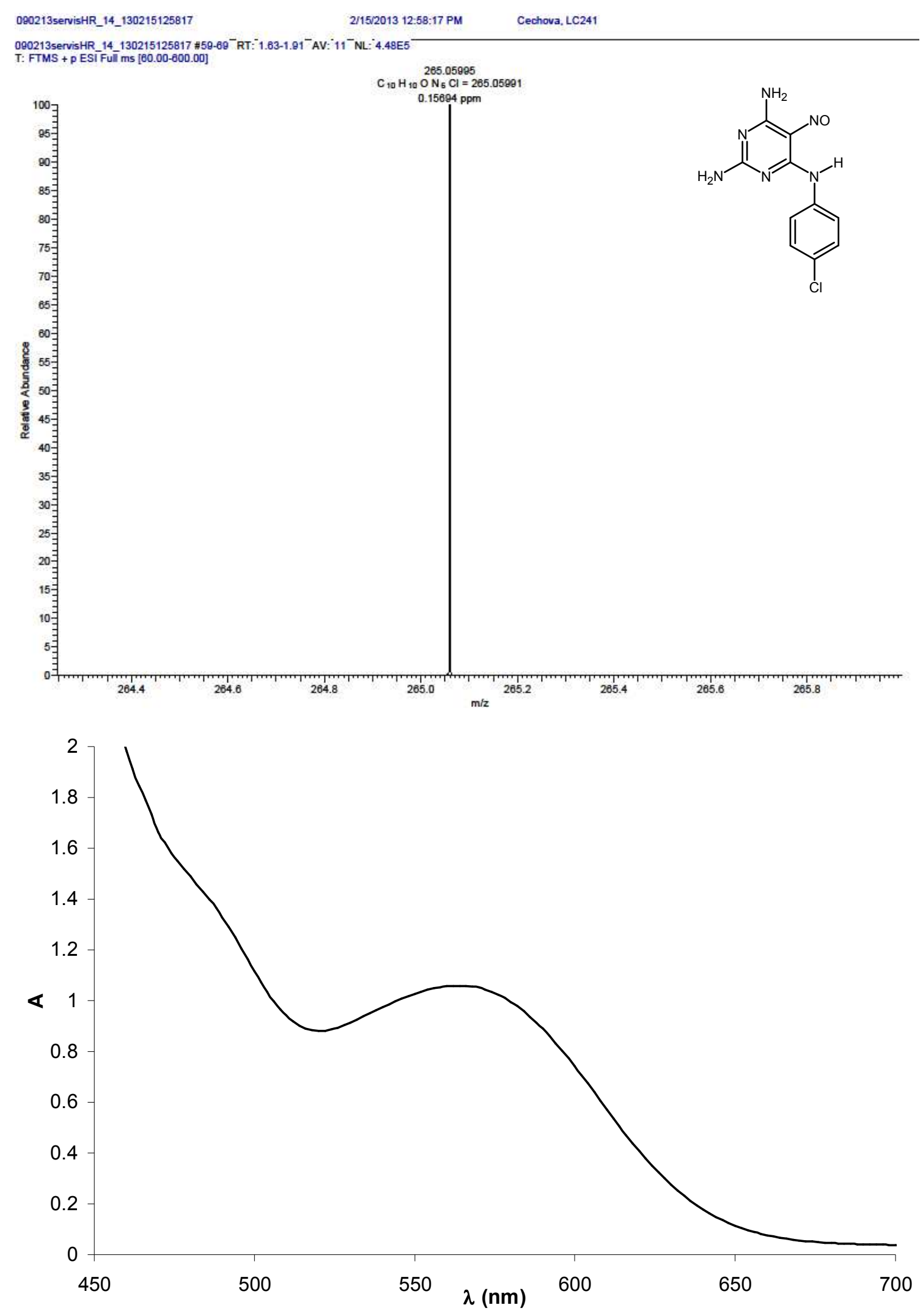

Figure S62. The HR-MS spectrum (top) and electronic spectrum (bottom) of compound $6 \mathbf{d}$. 


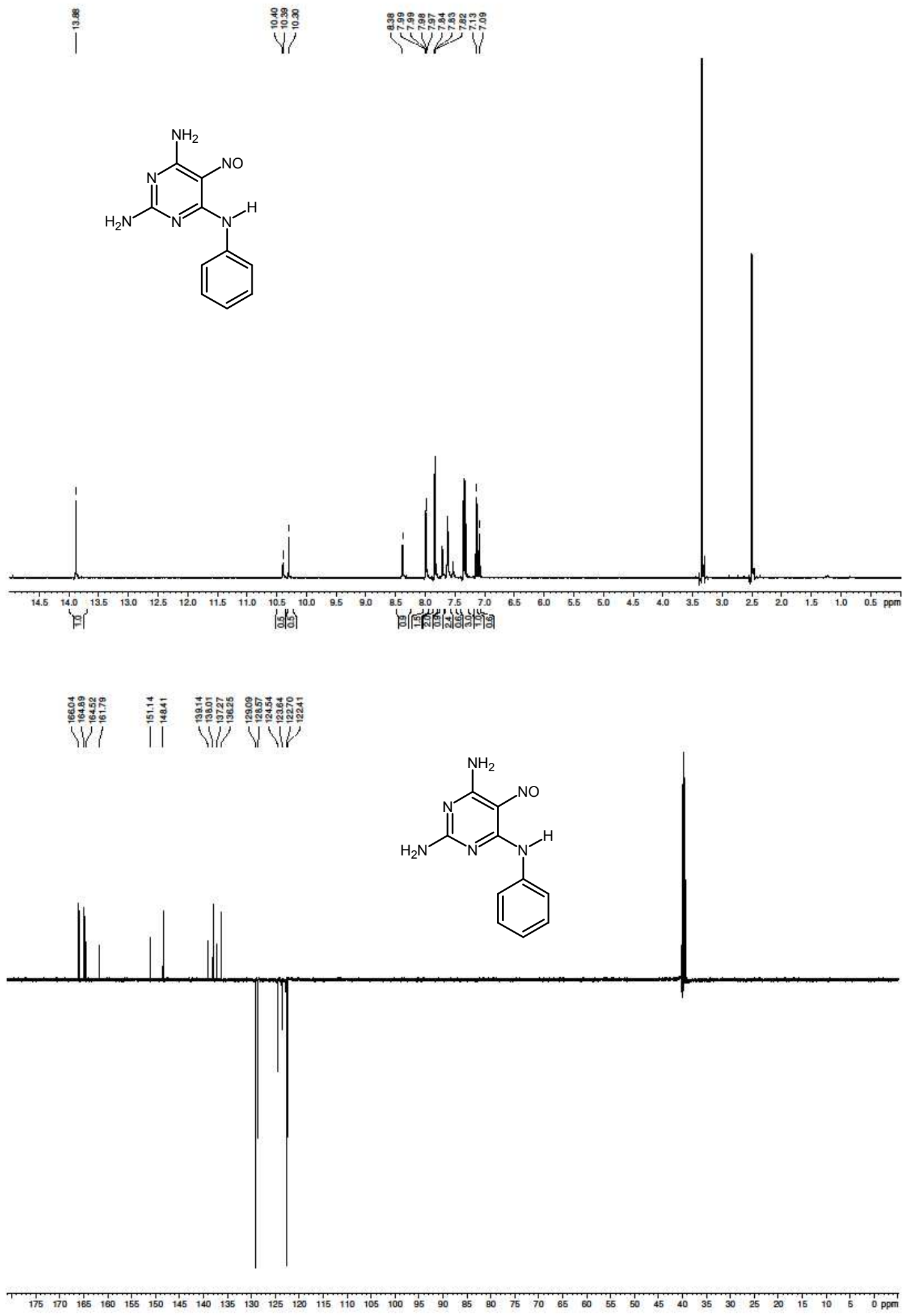

Figure S63. The NMR spectra of compound $\mathbf{6 e .}$ 

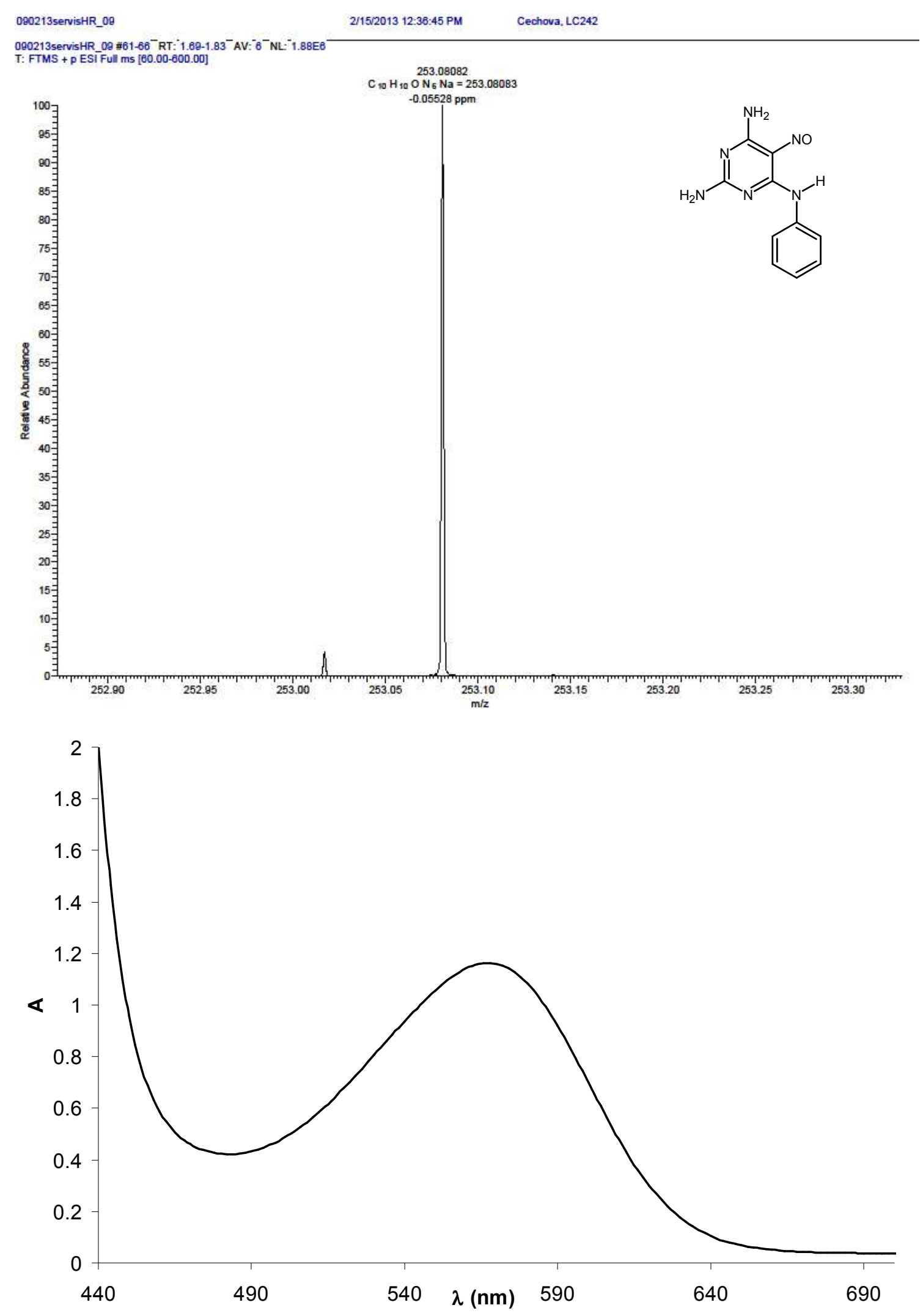

Figure S64. The HR-MS spectrum (top) and electronic spectrum (bottom) of compound $6 \mathbf{e}$. 

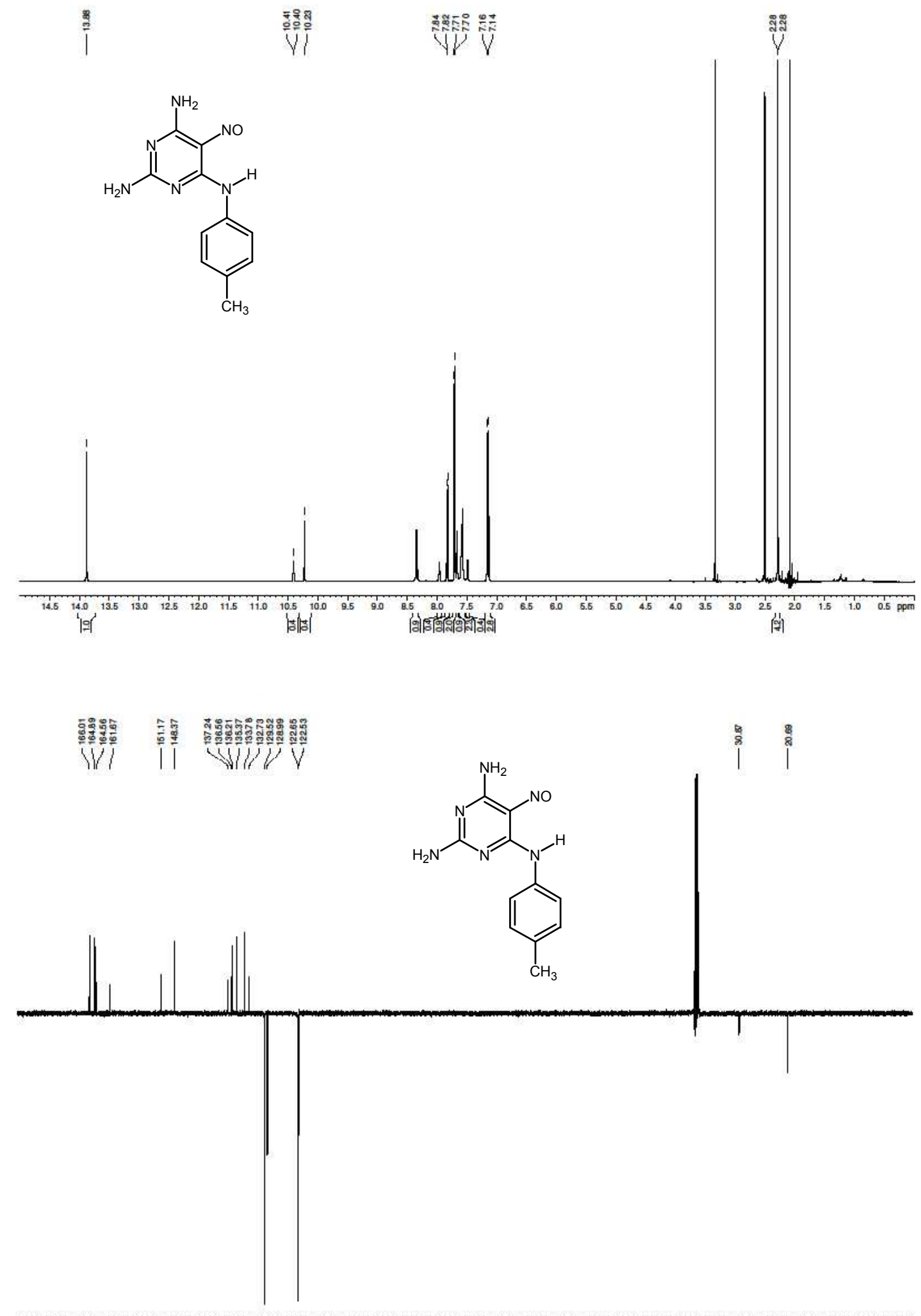

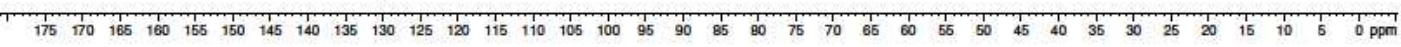

Figure S65. The NMR spectra of compound $6 \mathbf{f}$. 


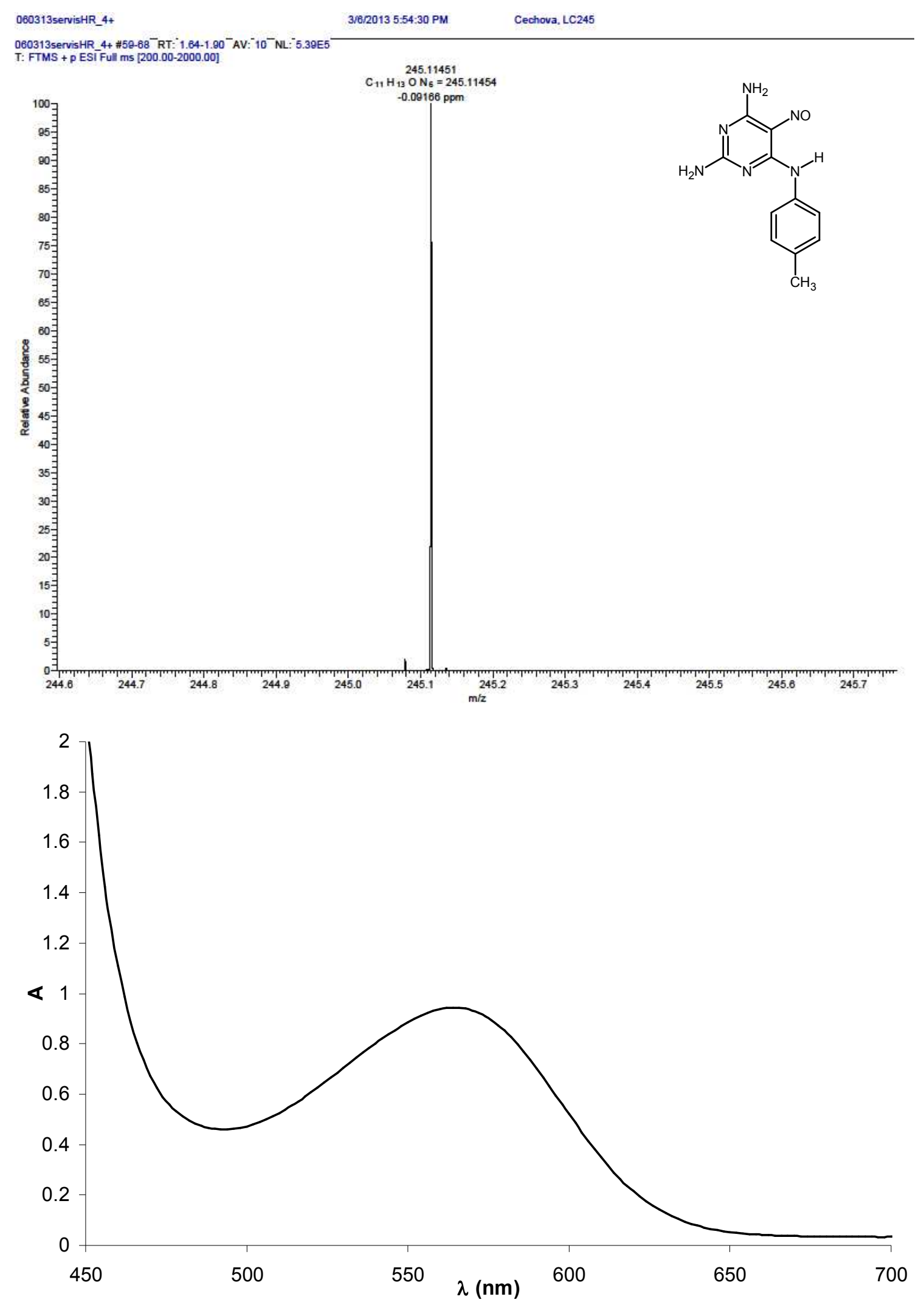

Figure S66. The HR-MS spectrum (top) and electronic spectrum (bottom) of compound $6 \mathbf{f}$. 

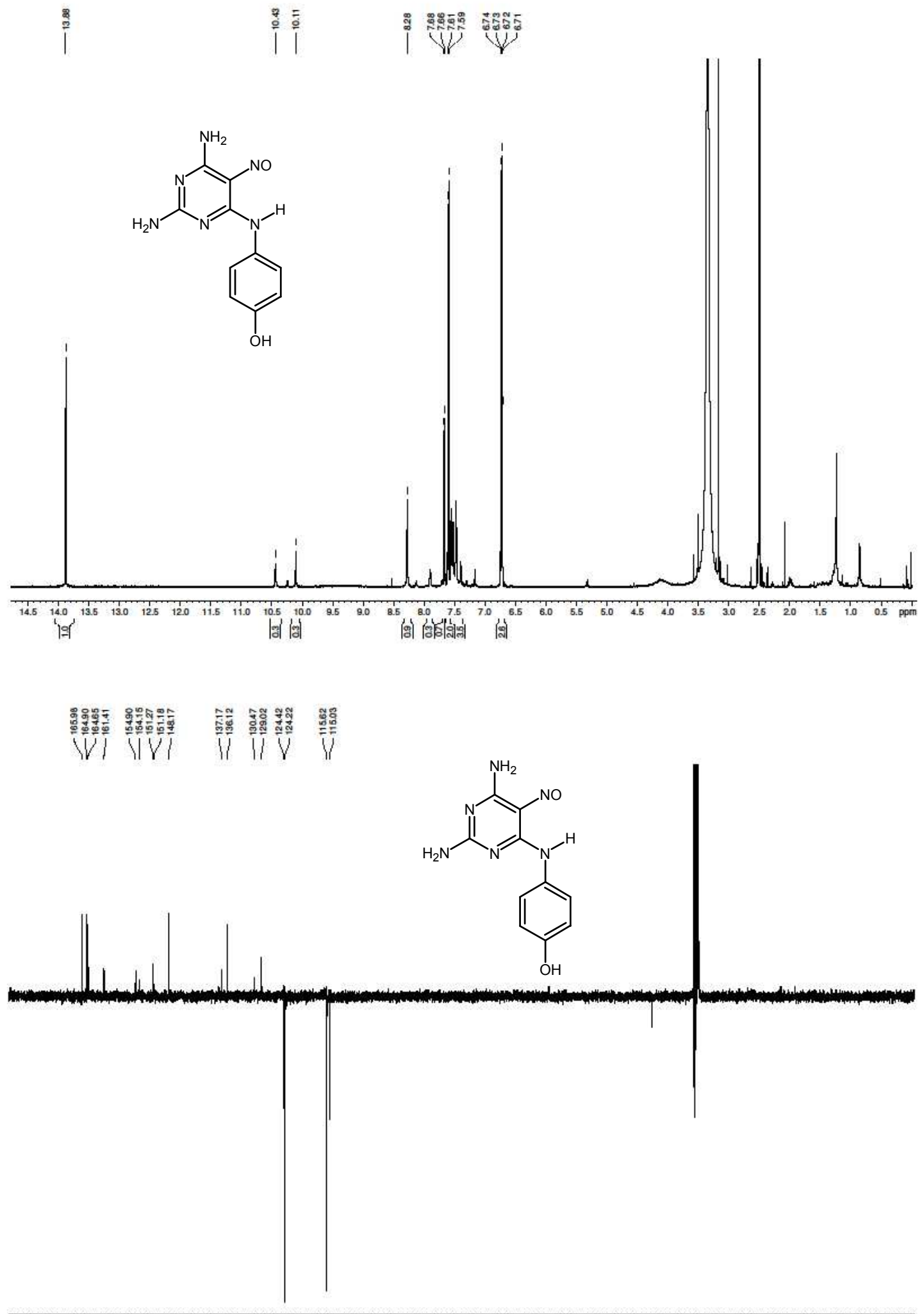

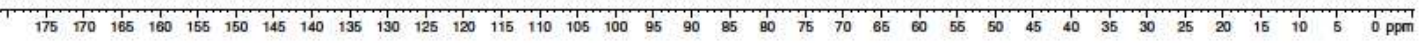

Figure S67. The NMR spectra of compound $\mathbf{6 g}$. 


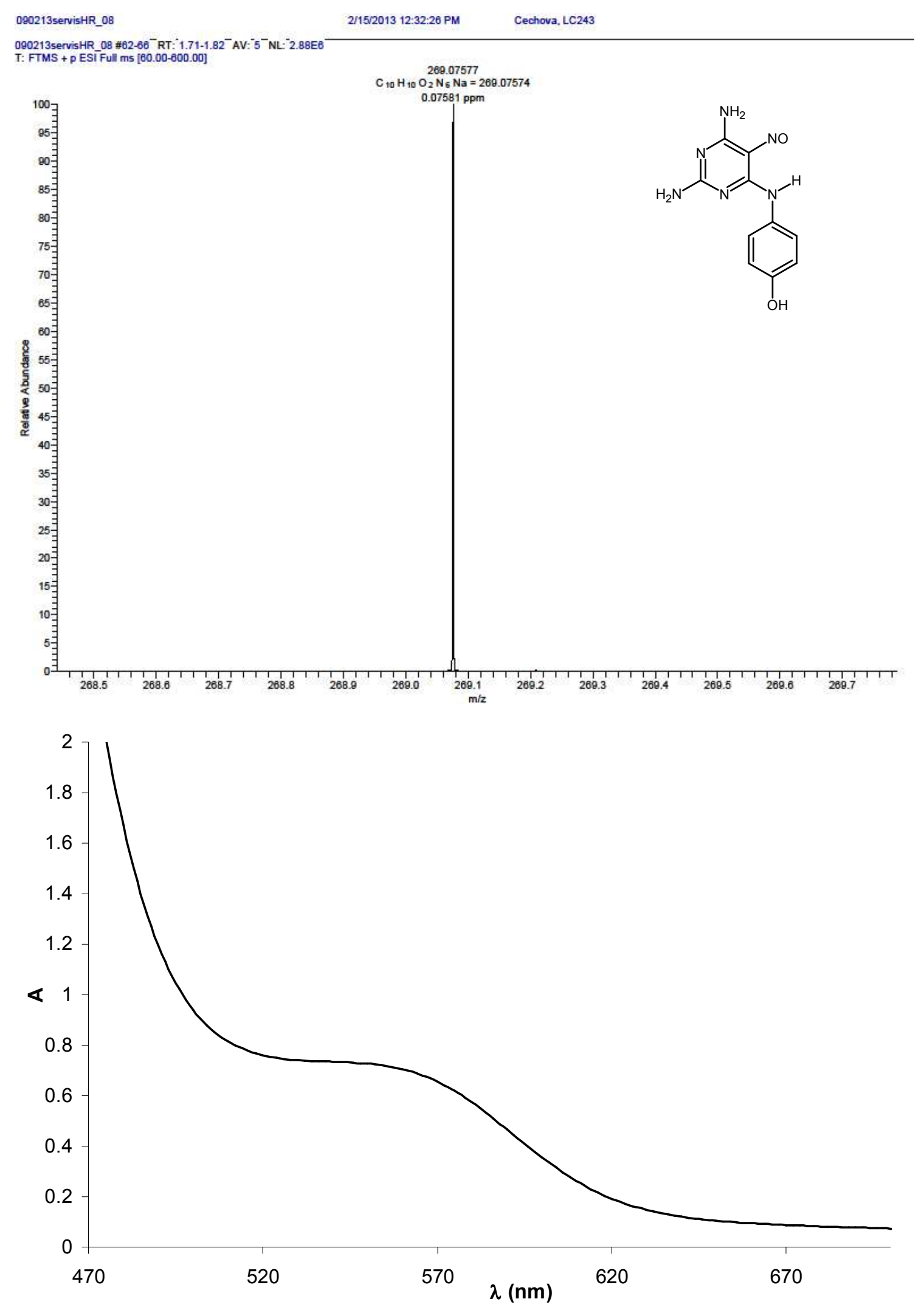

Figure S68. The HR-MS spectrum (top) and electronic spectrum (bottom) of compound $\mathbf{6 g}$. 

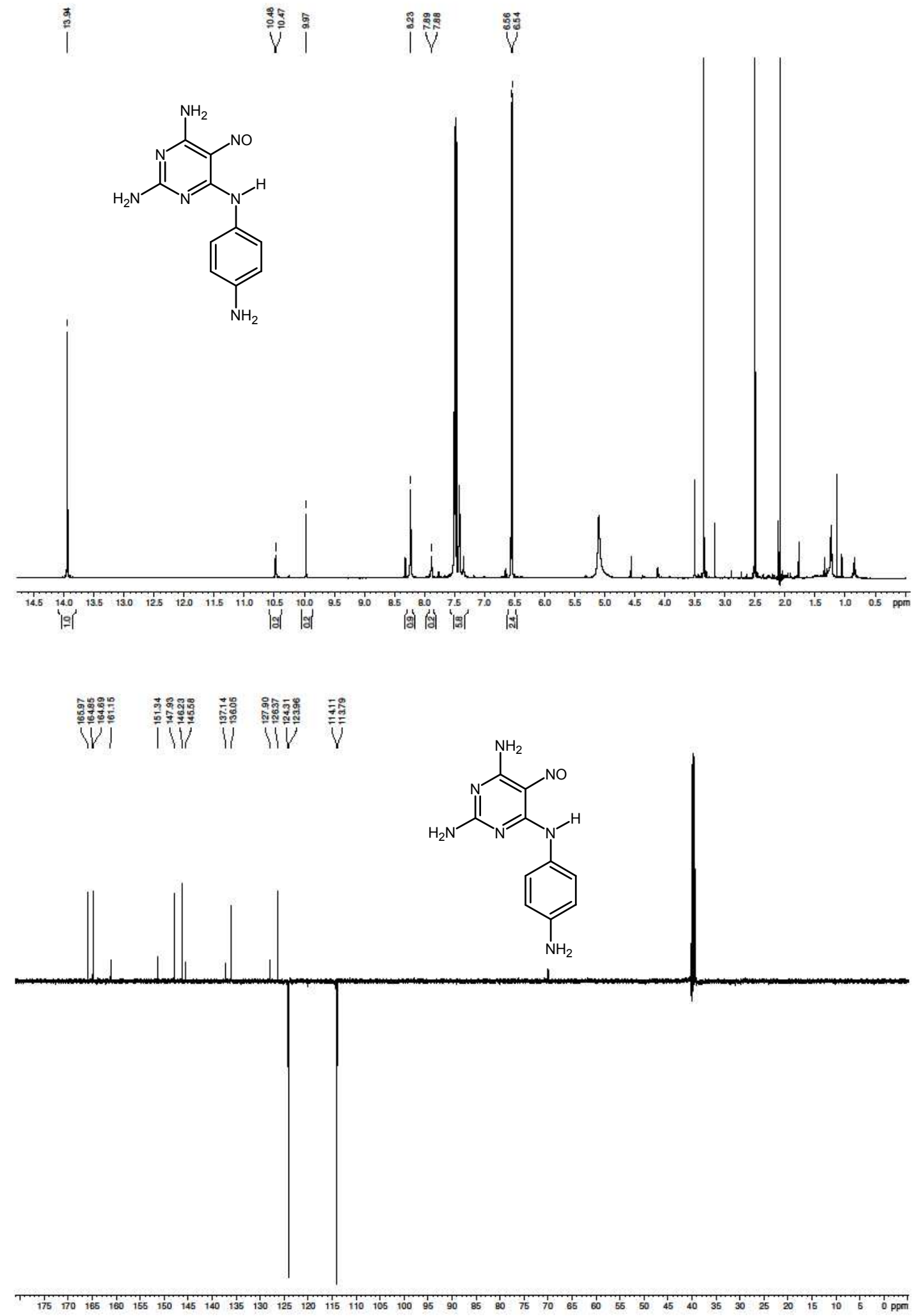

Figure S69. The NMR spectra of compound $\mathbf{6 h}$. 


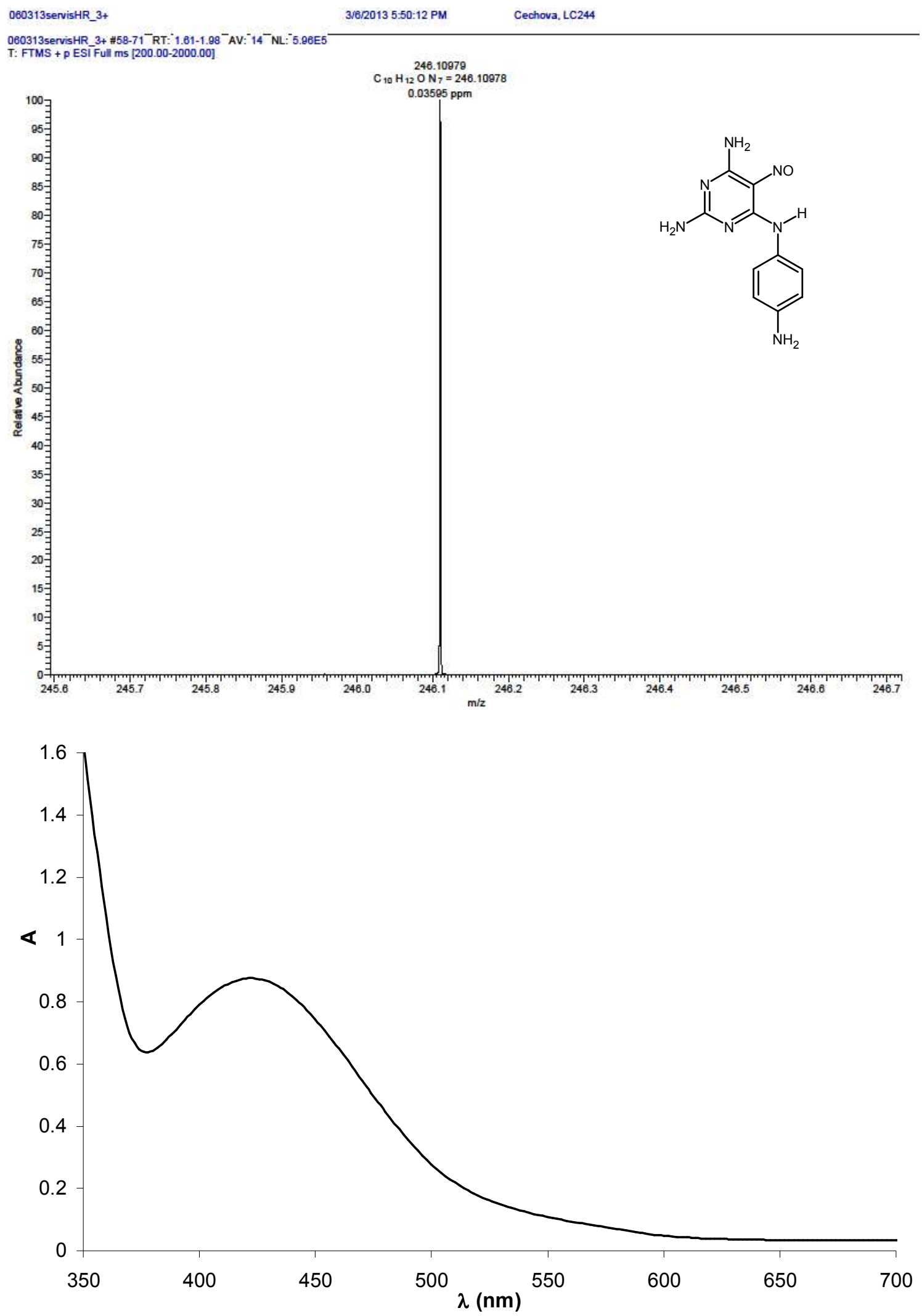

Figure S70. The HR-MS spectrum (top) and electronic spectrum (bottom) of compound $\mathbf{6 h}$.

73 

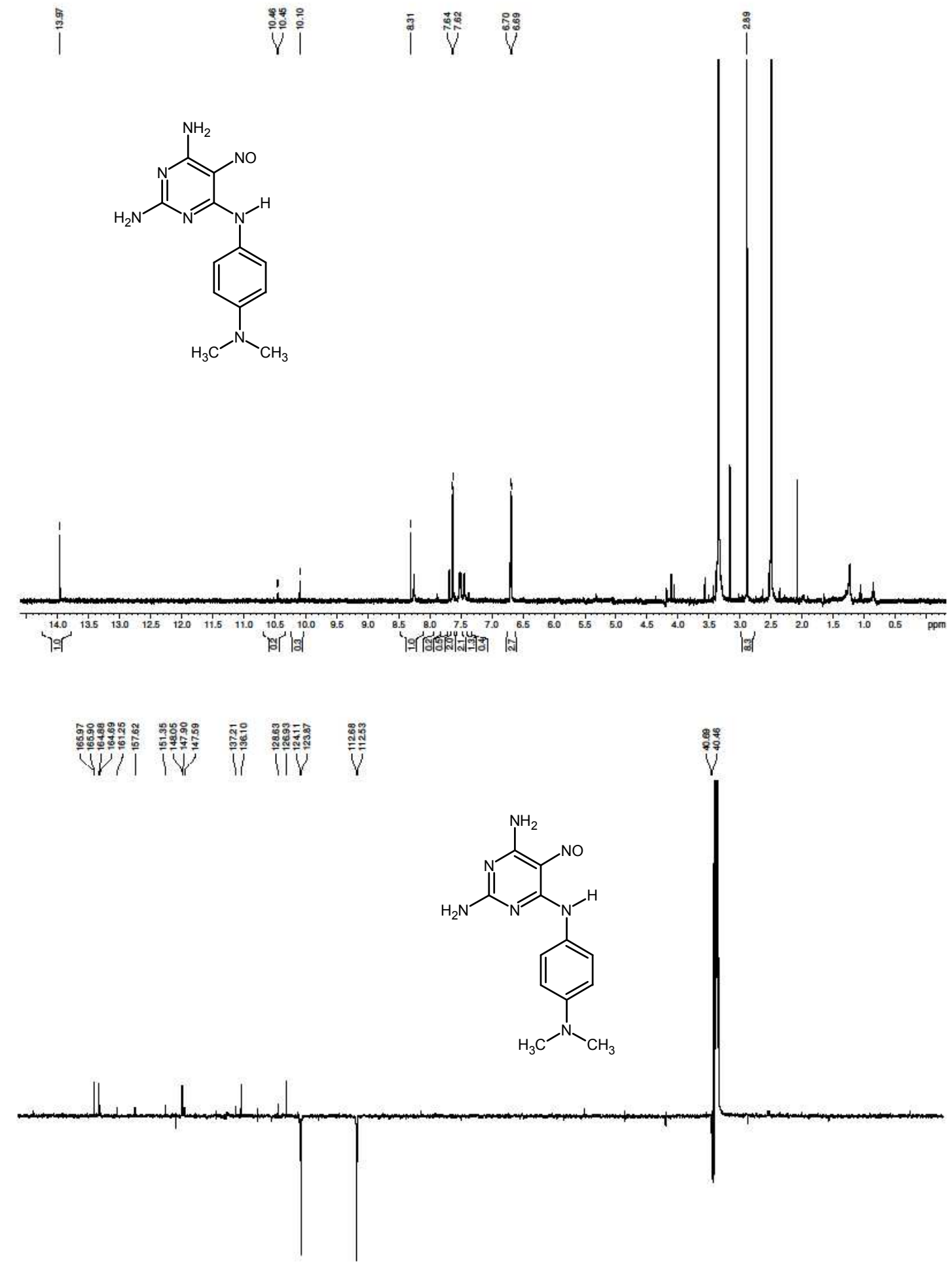

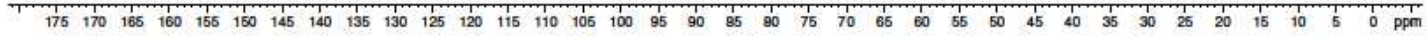

Figure S71. The NMR spectra of compound $\mathbf{6 i}$. 

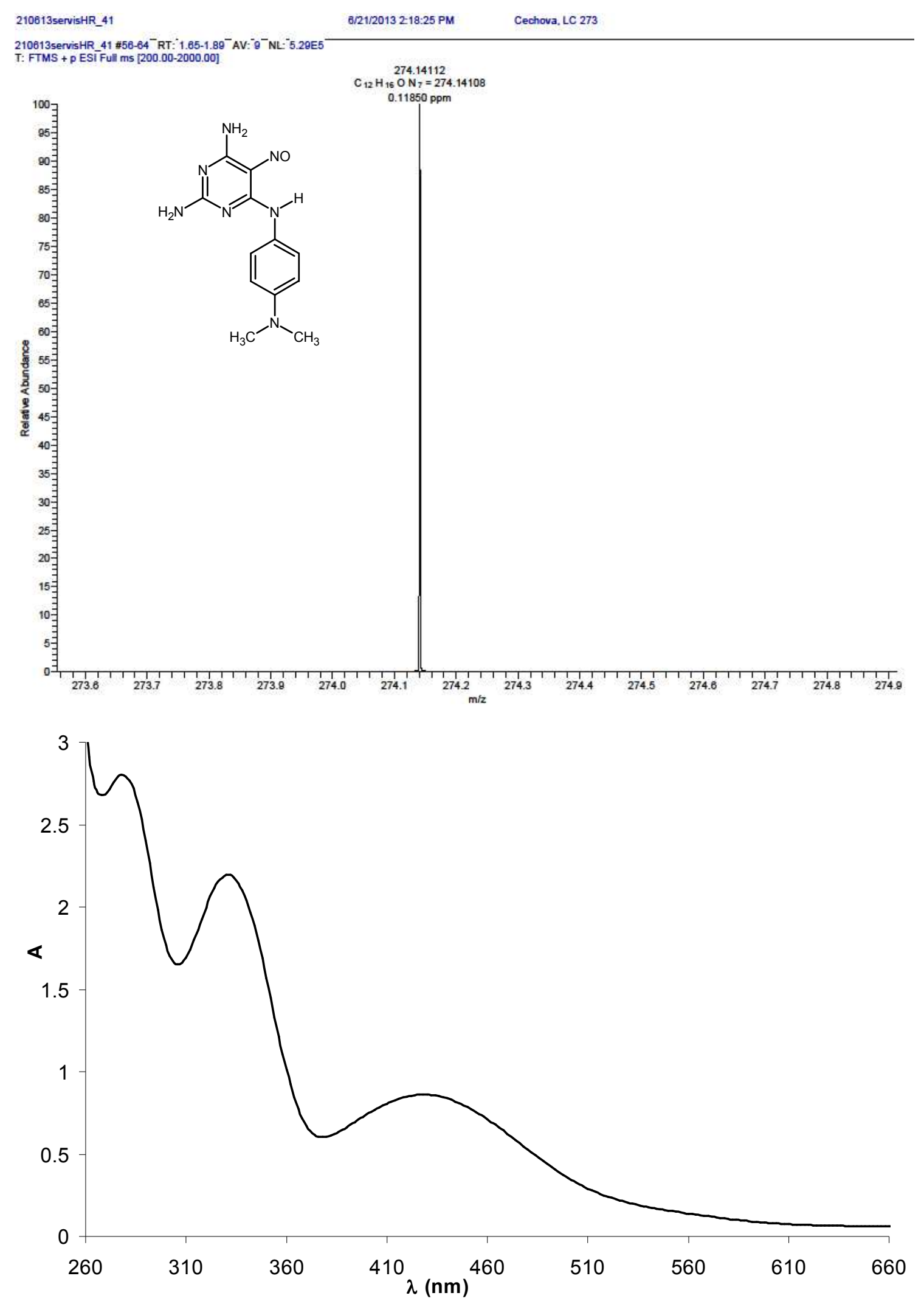

Figure S72. The HR-MS spectrum (top) and electronic spectrum (bottom) of compound $6 \mathbf{i}$. 

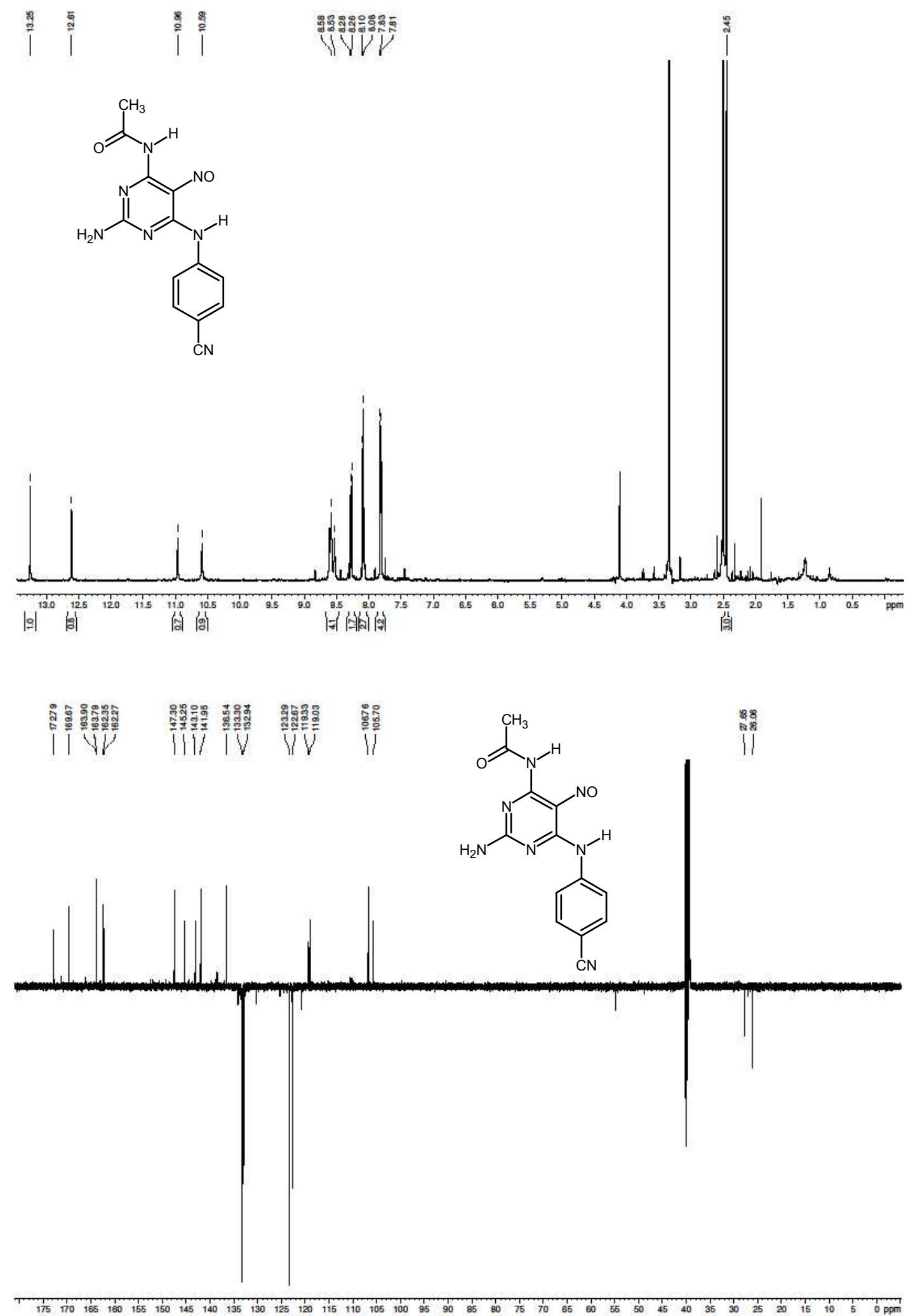

Figure S73. The NMR spectra of compound 7a. 


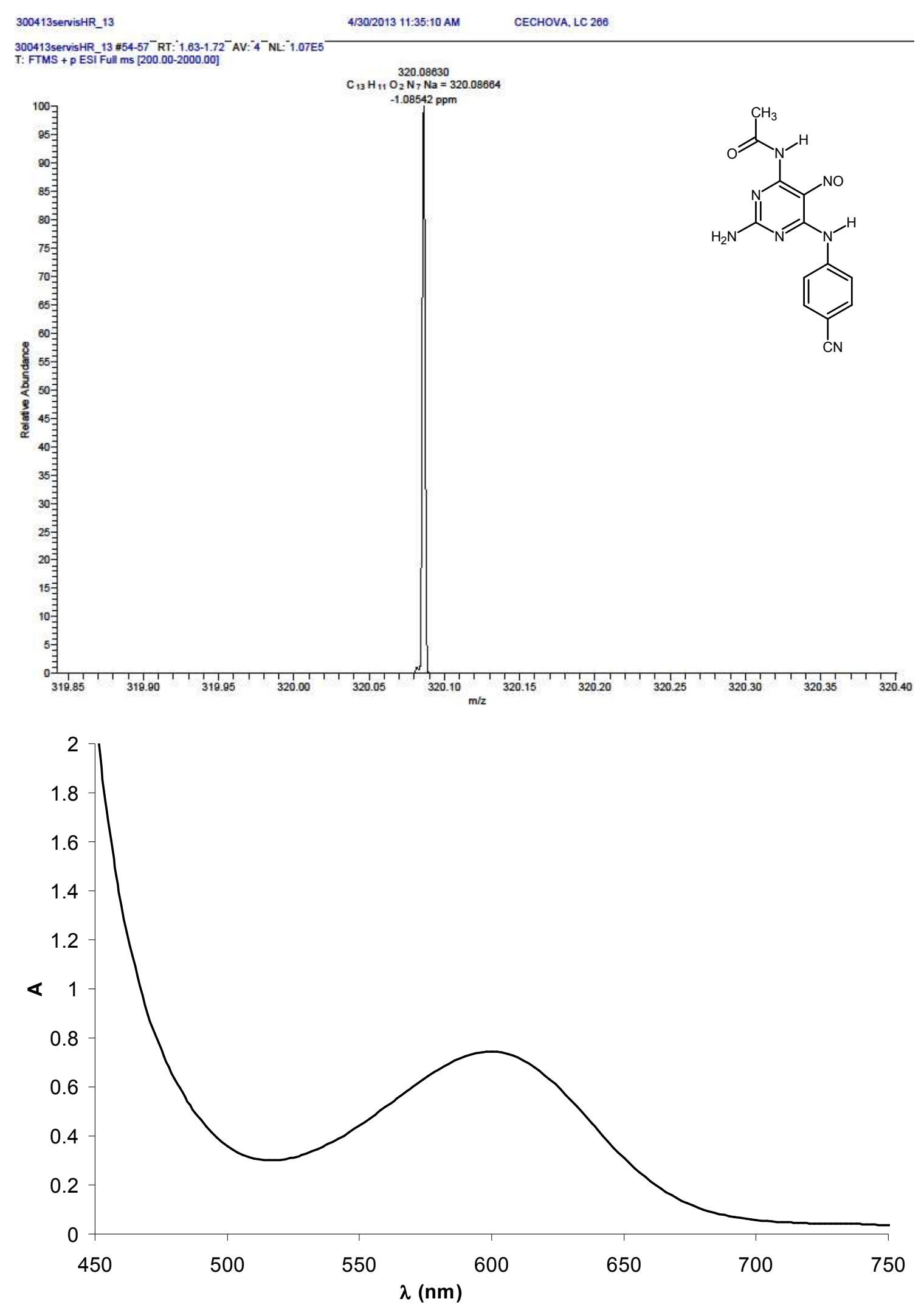

Figure S74. The HR-MS spectrum (top) and electronic spectrum (bottom) of compound 7a. 

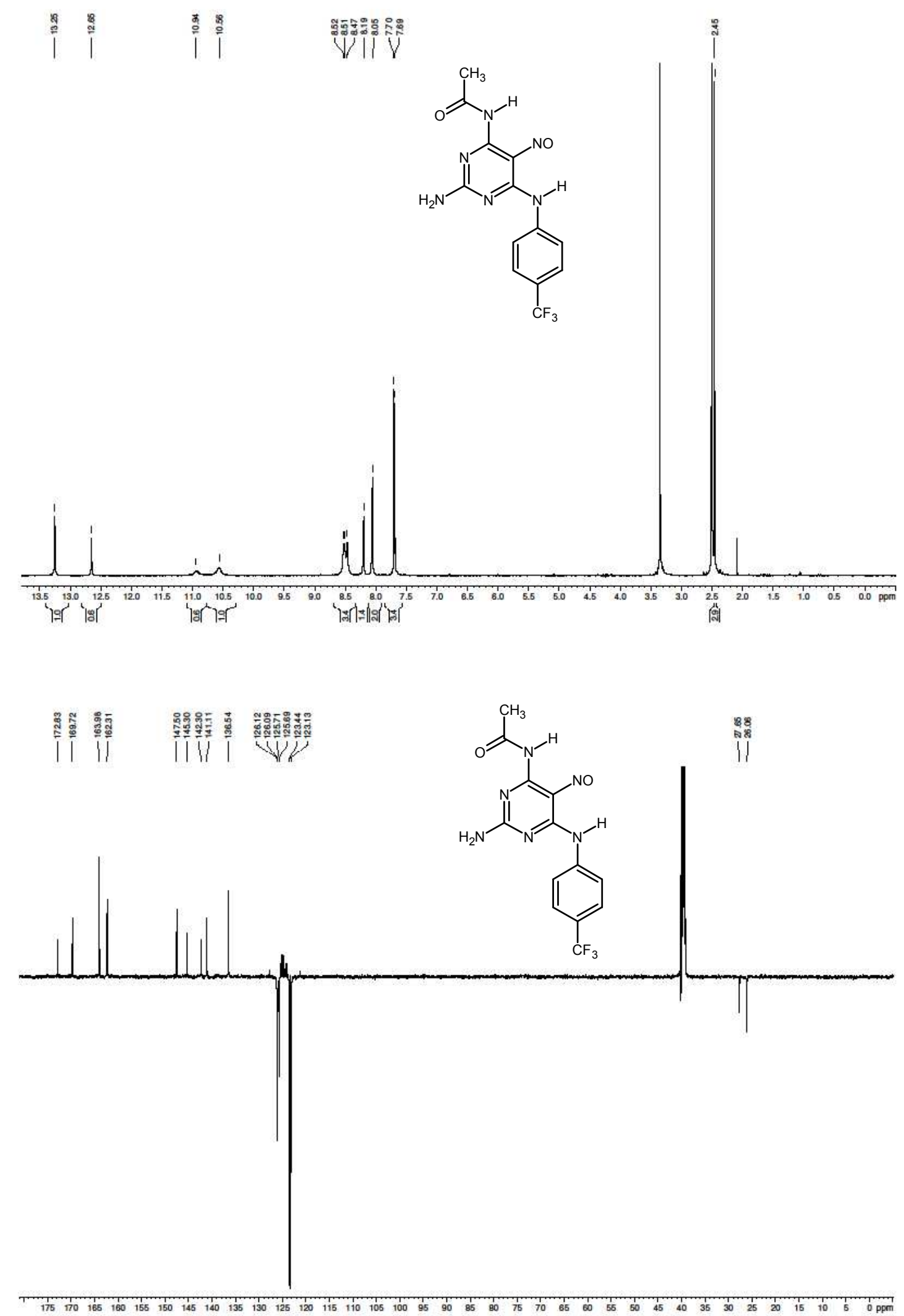

Figure S75. The NMR spectra of compound $7 \mathbf{b}$. 

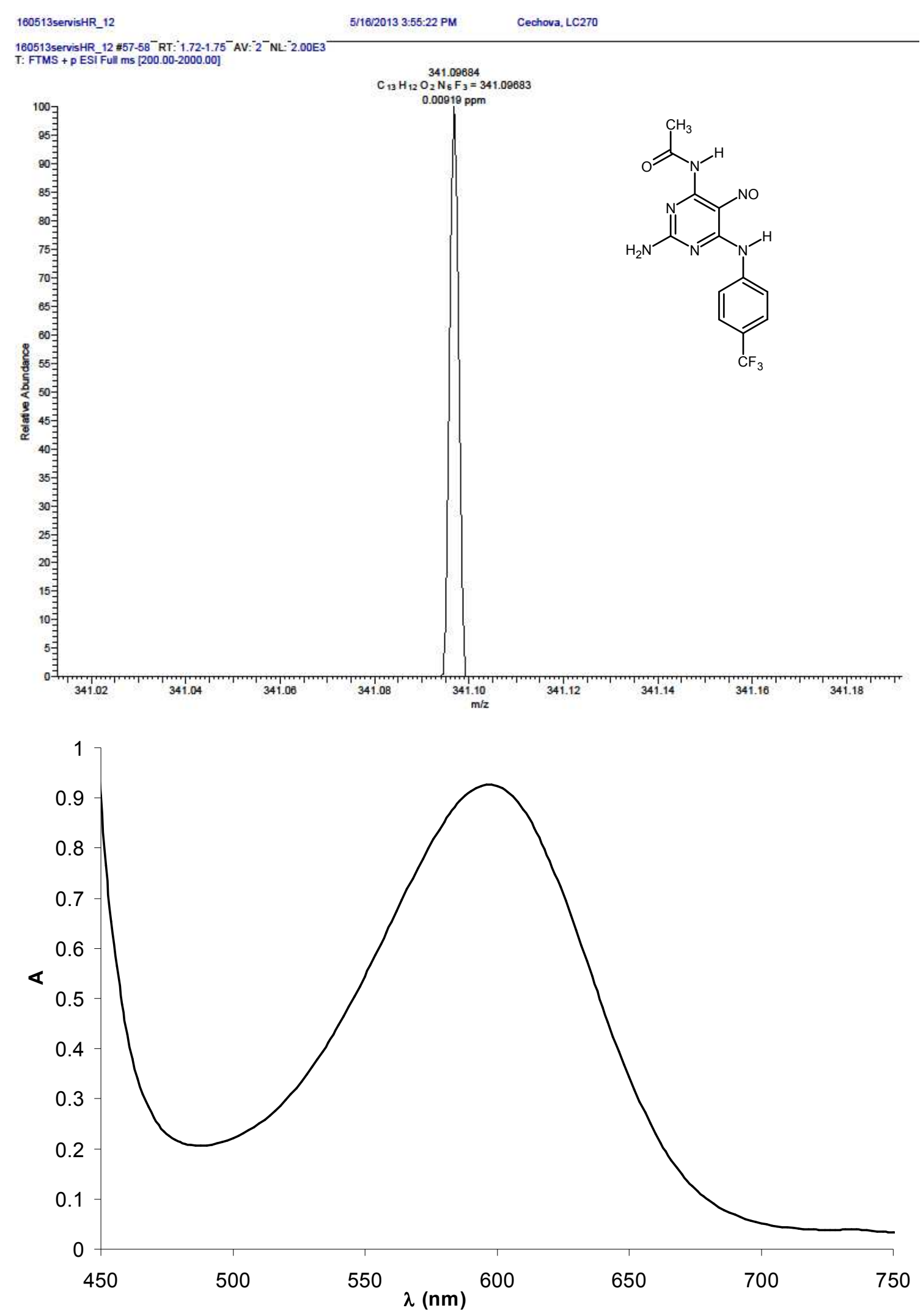

Figure S76. The HR-MS spectrum (top) and electronic spectrum (bottom) of compound 7b. 

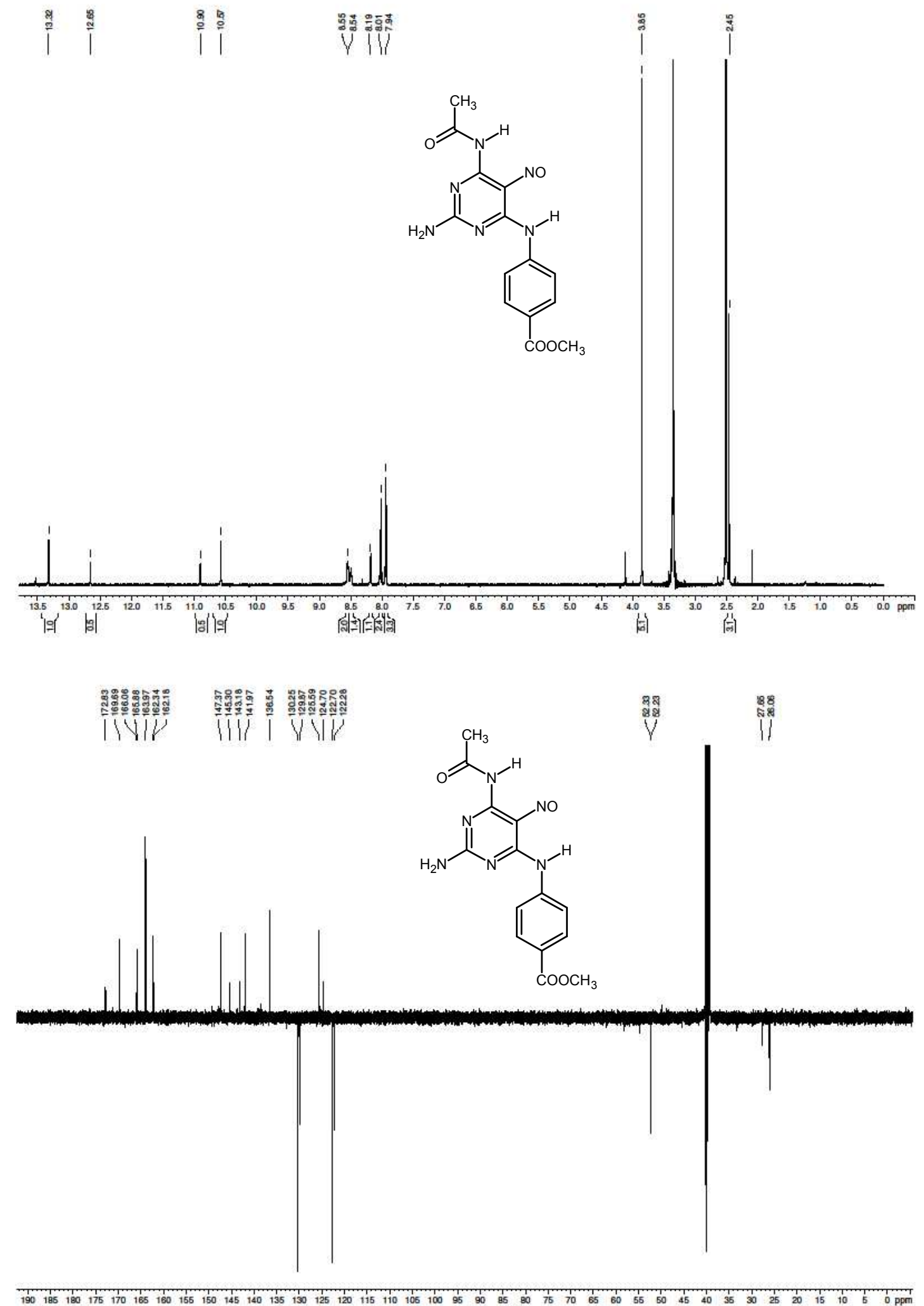

Figure S77. The NMR spectra of compound 7c. 

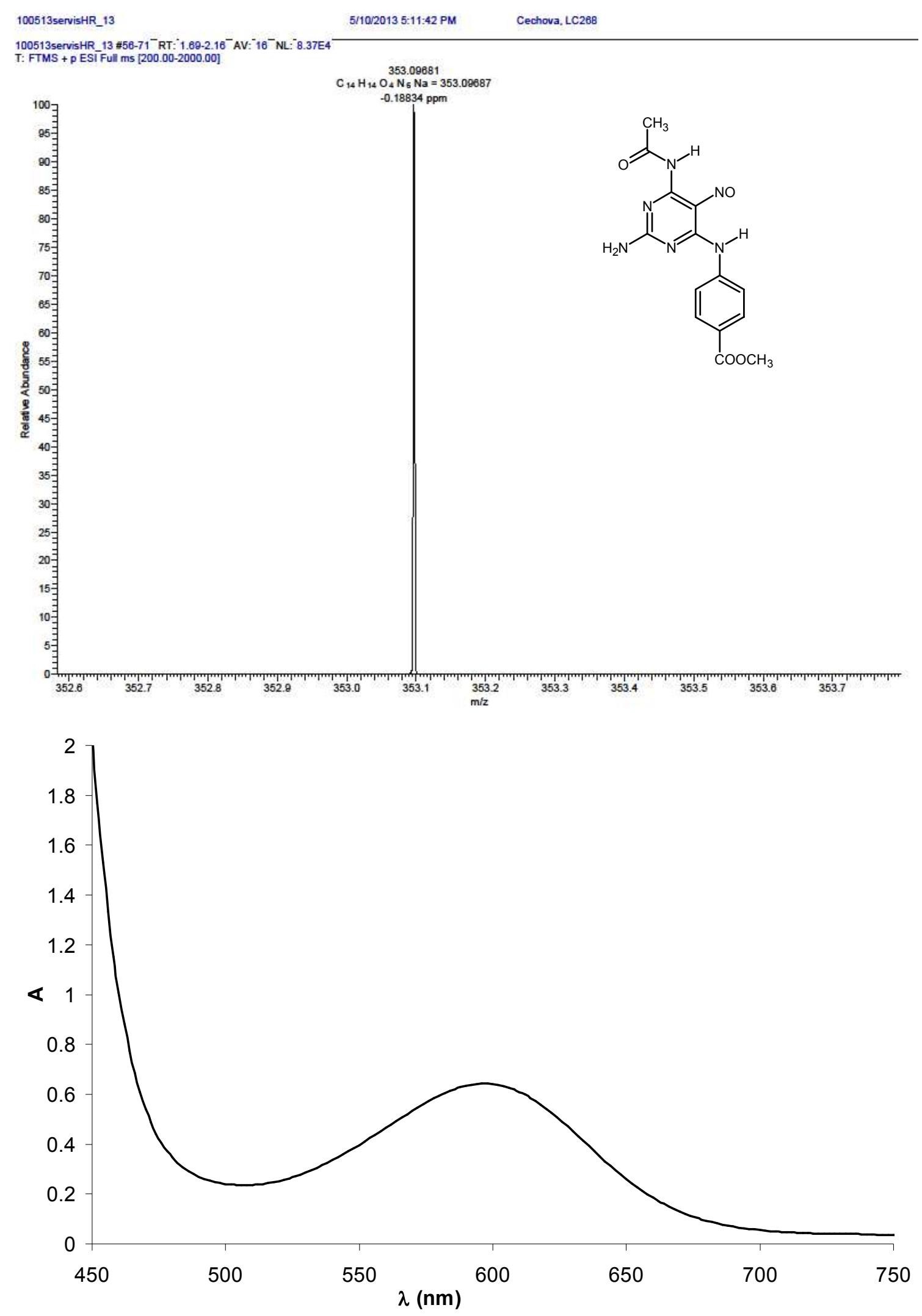

Figure S78. The HR-MS spectrum (top) and electronic spectrum (bottom) of compound 7c. 


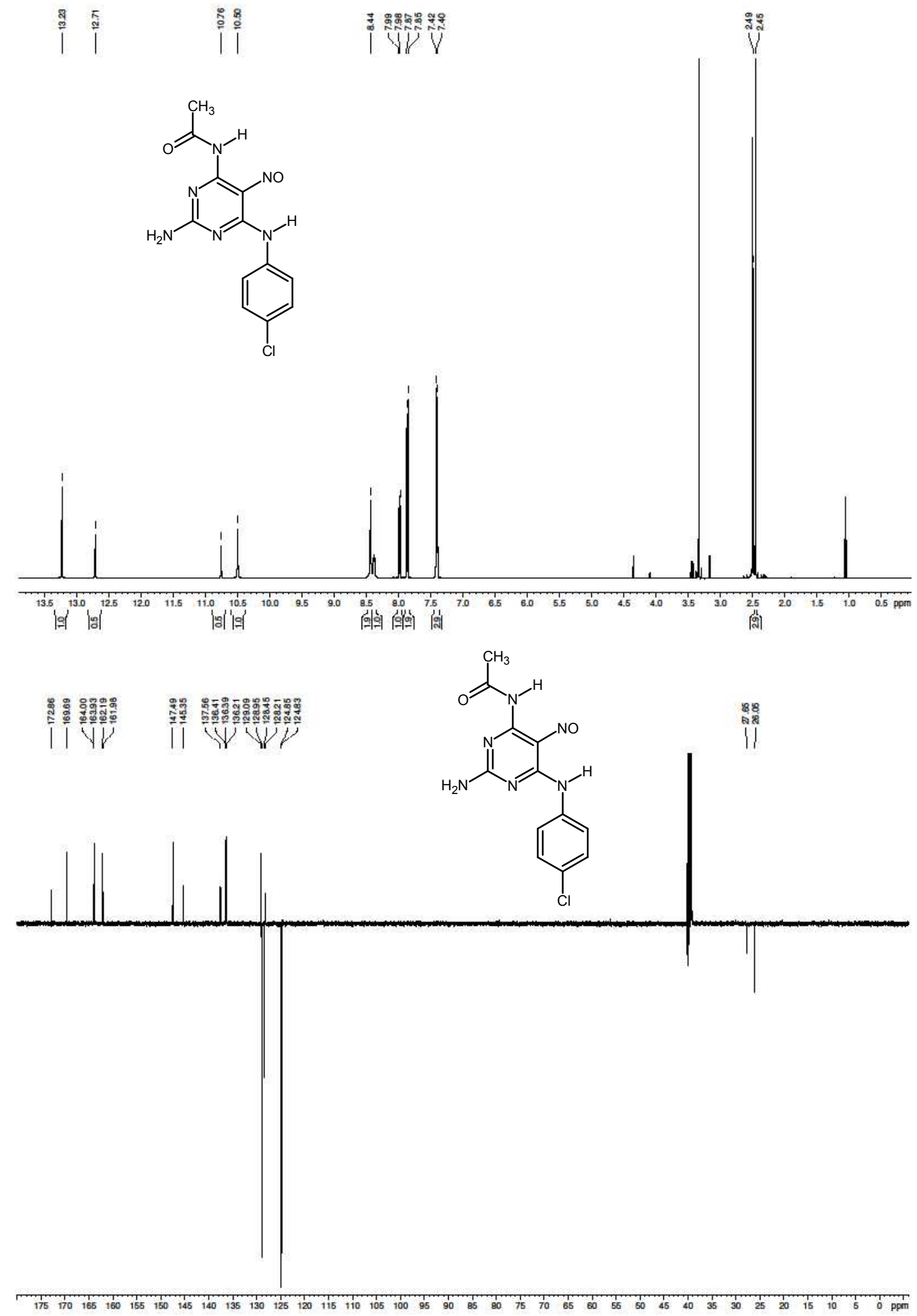

Figure S79. The NMR spectra of compound 7d. 

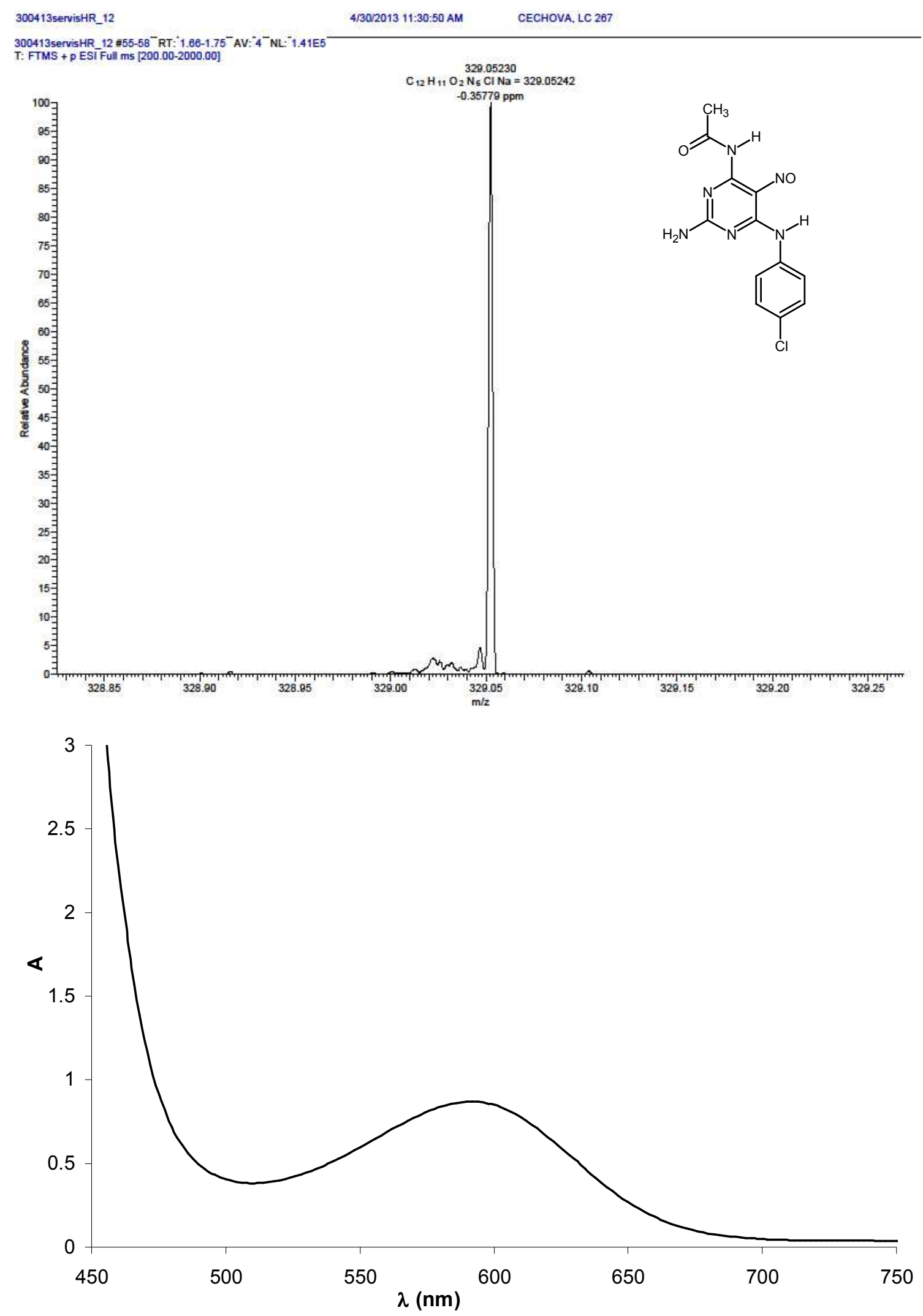

Figure S80. The HR-MS spectrum (top) and electronic spectrum (bottom) of compound 7d. 

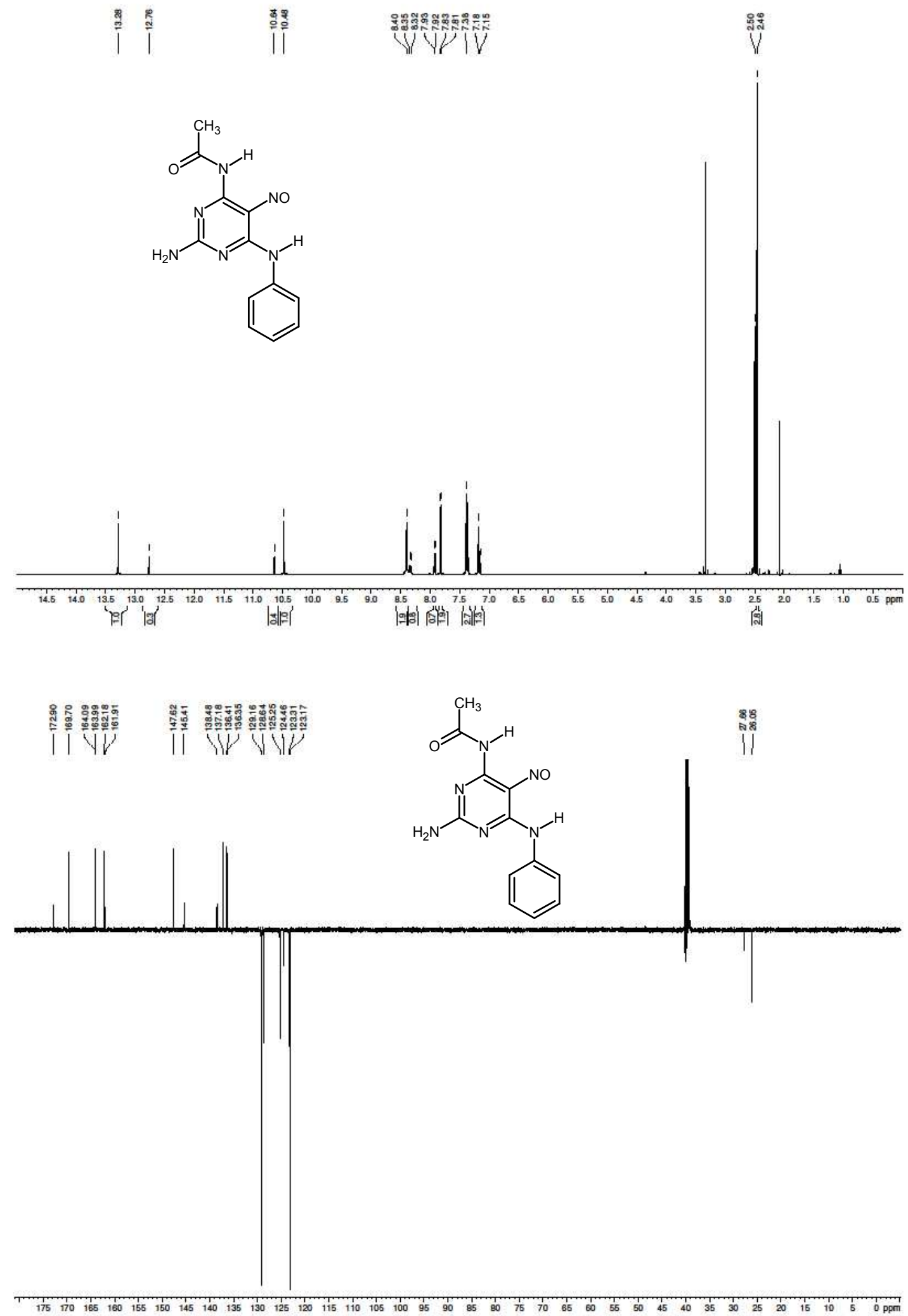

Figure S81. The NMR spectra of compound 7e. 

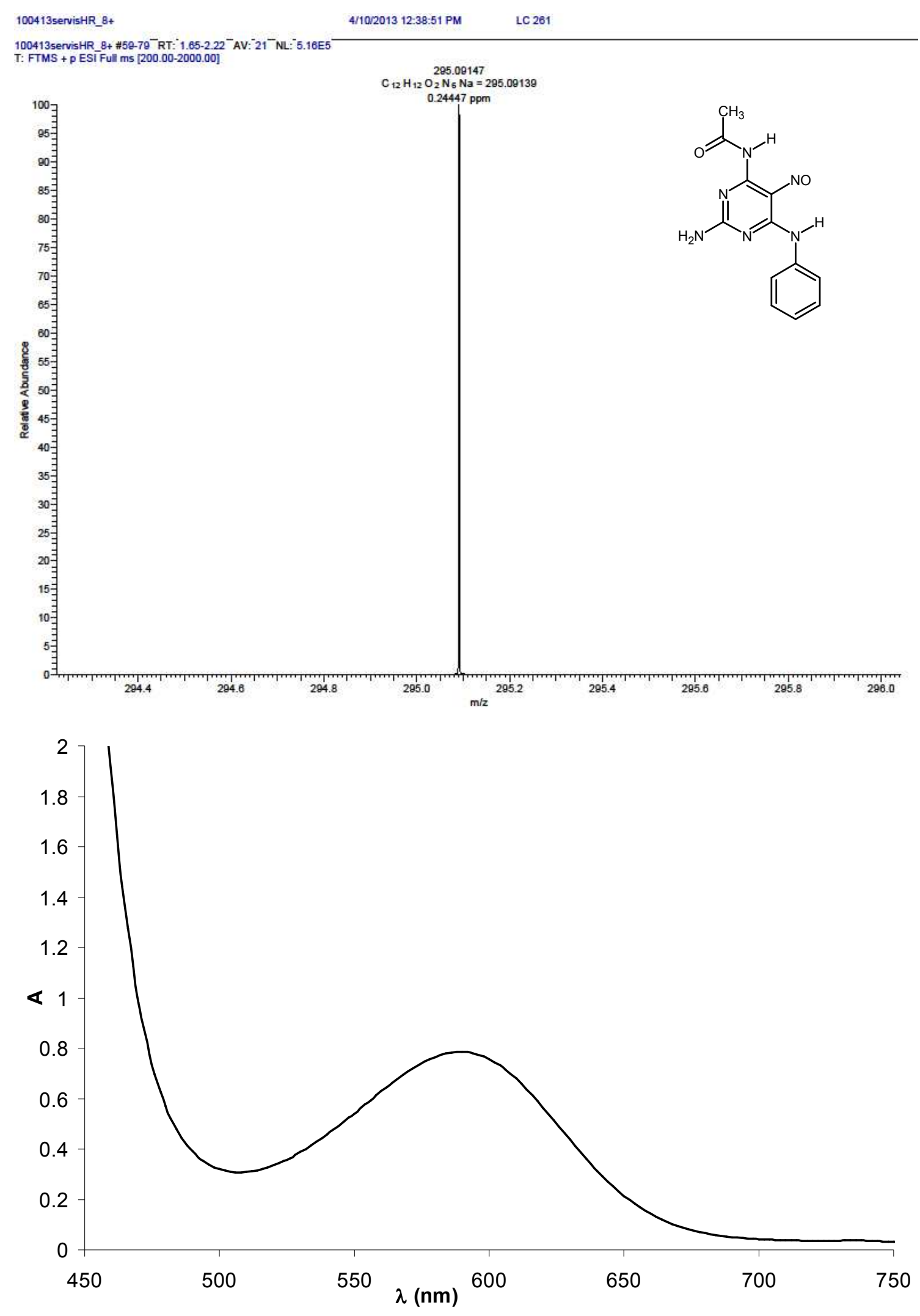

Figure S82. The HR-MS spectrum (top) and electronic spectrum (bottom) of compound 7e. 


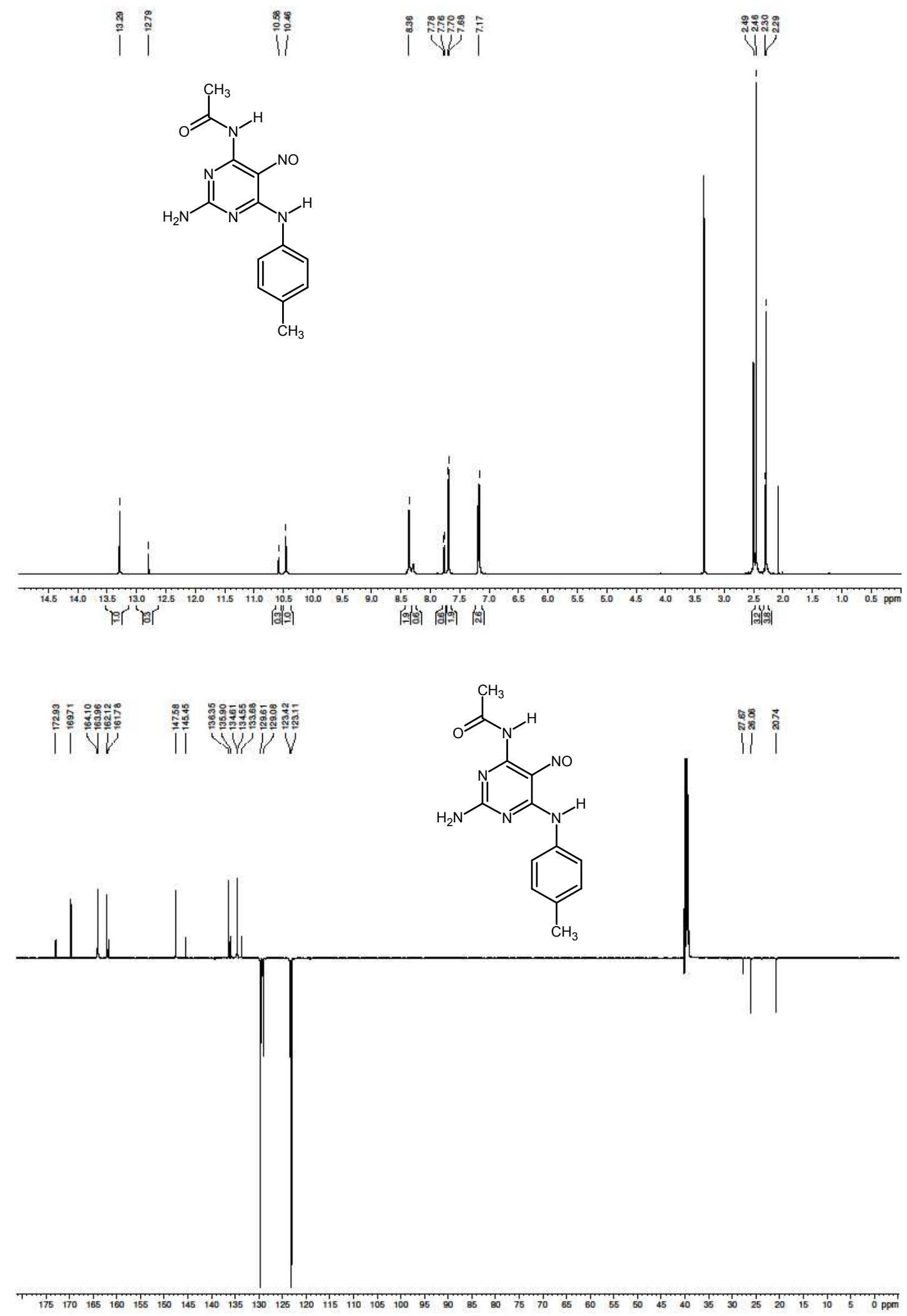

Figure S83. The NMR spectra of compound $7 \mathbf{f}$. 


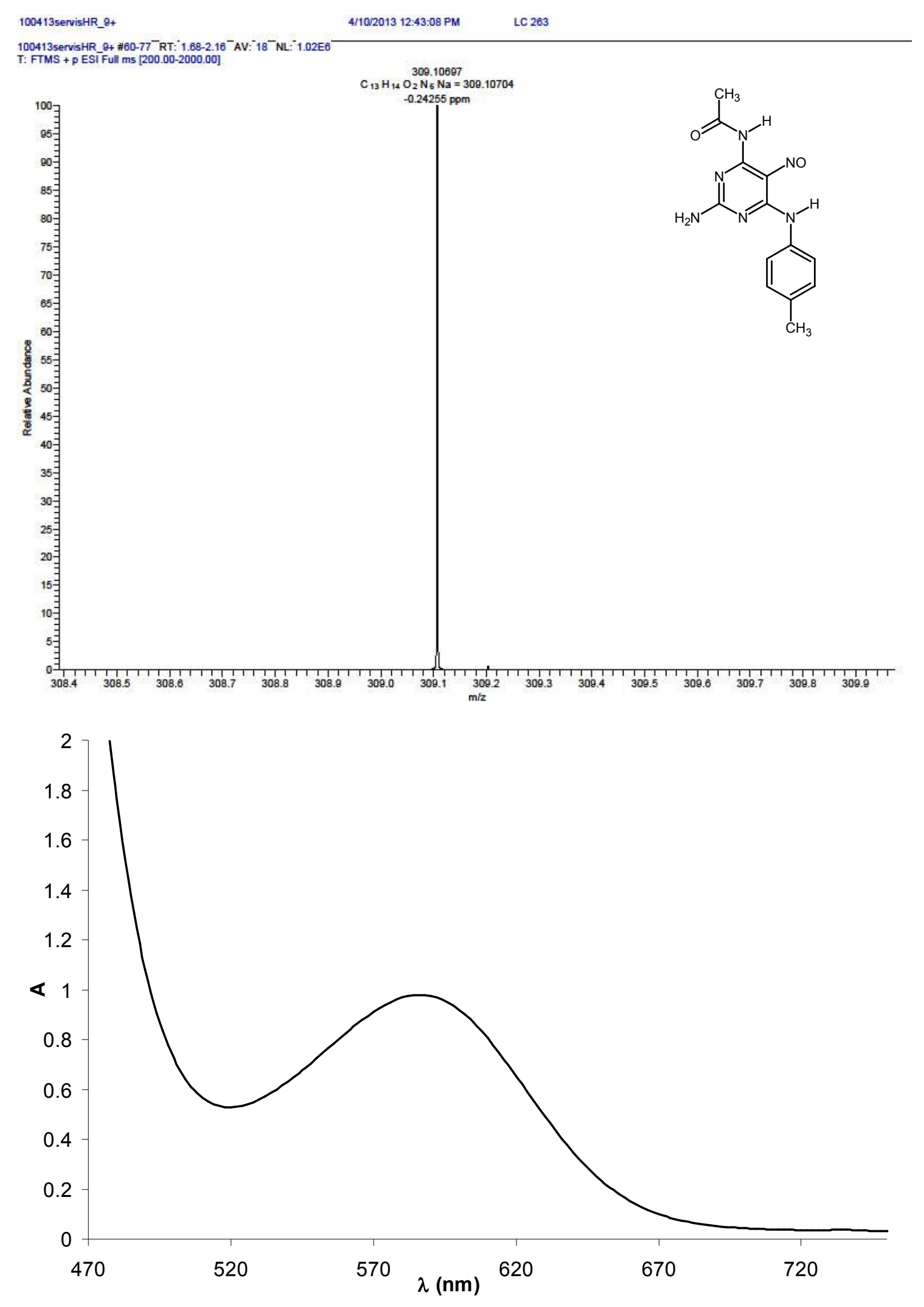

Figure S84. The HR-MS spectrum (top) and electronic spectrum (bottom) of compound 7f. 


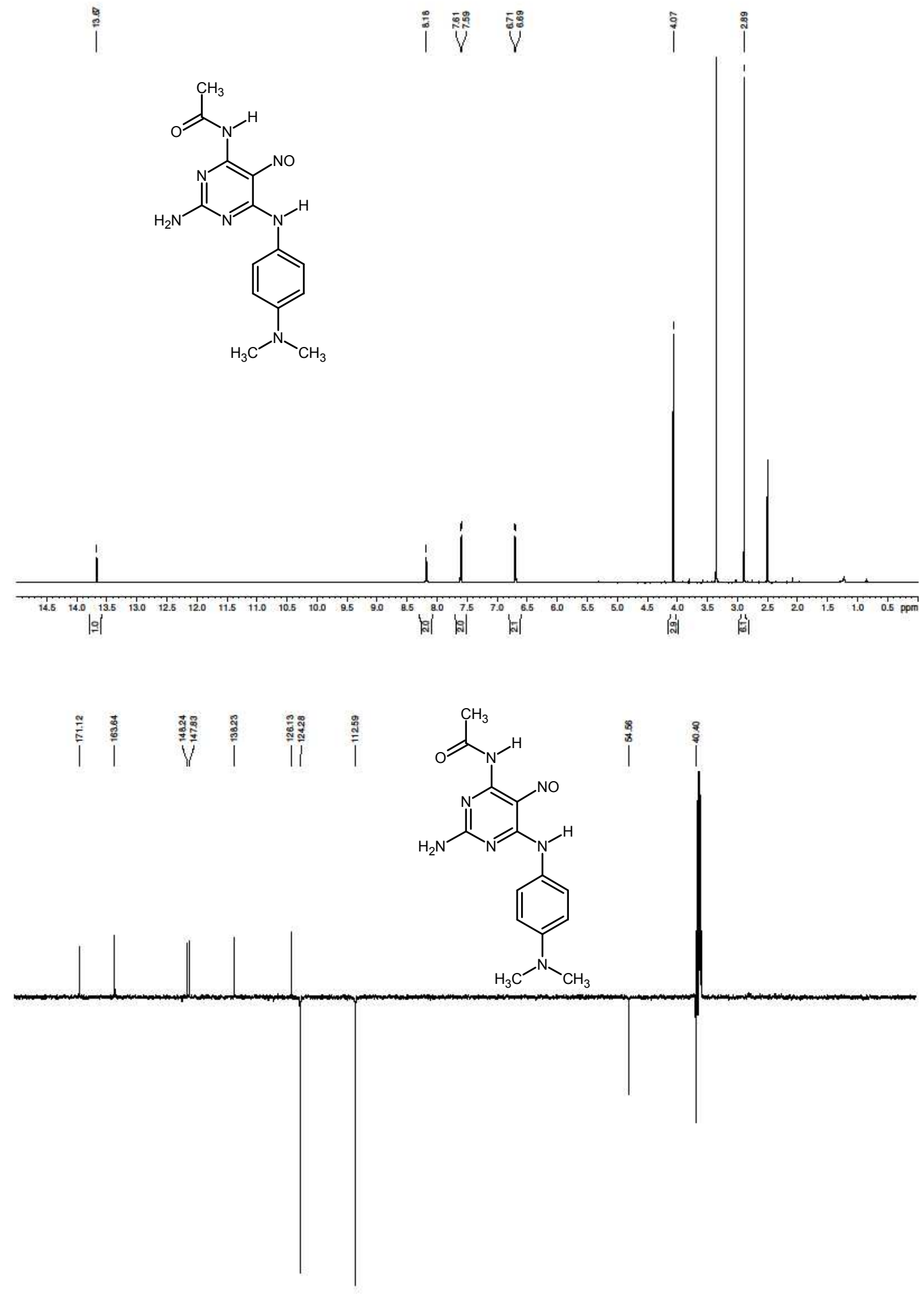

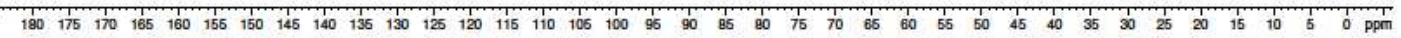

Figure S85. The NMR spectra of compound $7 \mathbf{i}$. 

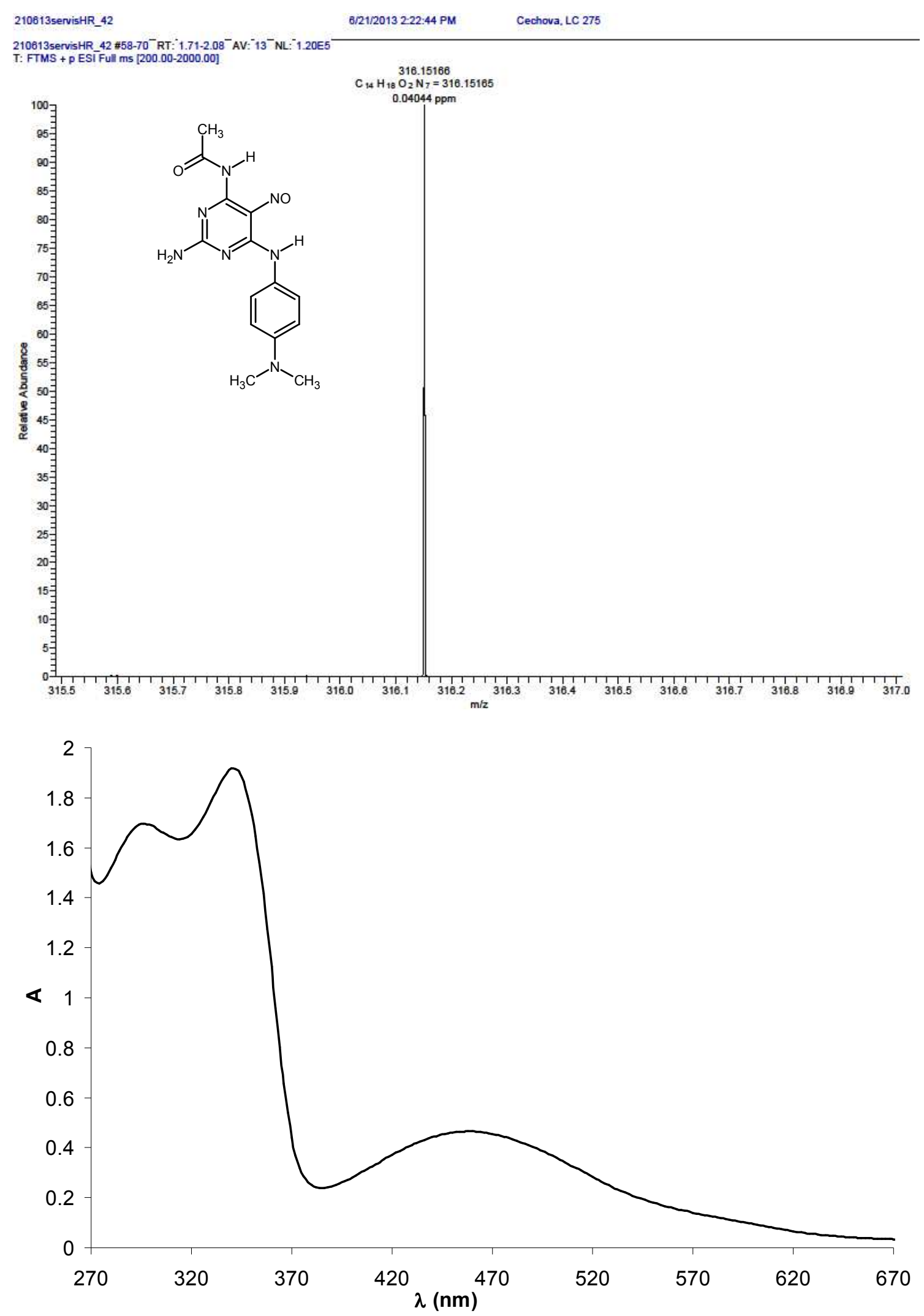

Figure 86: The HR-MS spectrum (top) and electronic spectrum (bottom) of compound 7i. 
Tables S3. Computational details: Cartesian coordinates of optimized structures

Compound 3a

Atomic number

6

6

6

7

7
6

6

8

8
7

6

1

$6 \quad 2.612267$

$6 \quad 3.971760$

$6 \quad 4.423894$

$6 \quad 2.115947$

$6 \quad 3.481535$

$7 \quad-2.008943$

$1 \quad 2.270679$

$1 \quad 4.688929$

$1 \quad 1.397652$

$1 \quad 3.824119$

$1 \quad-2.882516$

$-1.132597$

$-5.634292$

$-5.681384$

$-6.440915$

$-5.681382$

5.826739

6.966662

$-4.428565$ $\mathbf{y}$

$-0.879134$

$-0.129664$

$-0.079134$

1.209437

1.242541

1.820957

$-2.241958$

$-2.953910$

$-0.837801$

$-0.442253$

$-1.851112$

$-1.487395$

$-1.215372$

0.117318

0.890248

1.158766

3.171278

$-2.518514$

1.695428

2.188305

3.672650

3.667426

0.074982

0.703836

$-0.657024$

0.703839

0.408461

0.646679

$-0.712792$
$-2.029131$

$\mathbf{Z}$
0.000000
0.000000
0.000000
0.000001
0.000000
0.000001
0.000000
0.000000
0.000000
0.000000
0.000000
0.000001
0.000001
0.000000
-0.000001
-0.000001
0.000001
0.000002
0.000002
-0.000002
-0.000003
0.000002
0.000002
-0.000001
0.891900
-0.000003
-0.891900
0.000000
0.000000
-0.000001

$-0.000001$

Compound $\mathbf{3 b}$

Atomic number

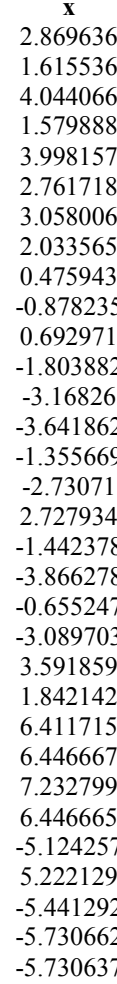

$\underset{-0.874178}{\mathbf{y}}$

$-0.148977$

$-0.05123$

1.190434

1.269726

1.824122

$-2.233189$

$-2.965679$

$-0.877772$

$-0.506934$

$-1.886918$

$-1.571011$

$-1.322665$

$-0.003361$

0.813448

1.053302

3.174477

$-2.595324$

$-2.15363$

1.634194

2.076437

3.692162

3.653487

0.148963

0.778798

$-0.566874$

0.778795

0.238125

$-0.662325$

1.555372

$-0.313869$

$-0.313775$
Z

$-0.000002$

$-0.000002$

0.000000

0.000000

0.000003

0.000003

$-0.000003$

$-0.000004$

$-0.000004$

$-0.000004$

$-0.000003$

0.000008

0.000008

$-0.000005$

$-0.000017$

$-0.000017$

0.000007

0.000018

0.000018

$-0.000027$

$-0.000028$

0.000012

0.000011

0.000003

$-0.891823$

0.000002

0.891831

0.000001

0.000000

$-0.000051$

$-1.087152$

1.087217

Compound 3c

Atomic number

$\mathbf{x}$
2.888841
1.650942
4.081478

y

$-0.893239$

$-0.139799$

$-0.096776$

0.000000

0.000000

0.000000 


$\begin{array}{lccc}7 & 1.646829 & 1.200268 & 0.000001 \\ 7 & 4.06611 & 1.224691 & 0.000000 \\ 6 & 2.841886 & 1.8068 & 0.000001 \\ 7 & 3.047506 & -2.255838 & 0.000000 \\ 8 & 2.007254 & -2.966003 & 0.000000 \\ 7 & 0.494646 & -0.841316 & 0.000000 \\ 6 & -0.851102 & -0.440824 & 0.000000 \\ 1 & 0.687128 & -1.85496 & 0.000000 \\ 6 & -1.799336 & -1.484228 & 0.000002 \\ 6 & -3.159241 & -1.207299 & 0.000001 \\ 6 & -3.610293 & 0.12276 & 0.000000 \\ 6 & -1.299188 & 0.892498 & -0.000002 \\ 6 & -2.665762 & 1.157702 & -0.000002 \\ 7 & 2.838481 & 3.157796 & 0.000002 \\ 1 & -1.457889 & -2.515729 & 0.000003 \\ 1 & -3.876424 & -2.019744 & 0.000003 \\ 1 & -0.580417 & 1.697171 & -0.000003 \\ 1 & -3.019688 & 2.183331 & -0.000003 \\ 1 & 3.713553 & 3.656253 & 0.000003 \\ 1 & 1.963181 & 3.655875 & 0.000003 \\ 6 & 6.452702 & 0.050476 & -0.000001 \\ 1 & 6.501606 & 0.679645 & 0.891698 \\ 8 & -5.859732 & -0.607192 & 0.000001 \\ 6 & -7.273267 & -0.337597 & 0.000000 \\ 1 & -7.754176 & -1.315524 & 0.000001 \\ 1 & -7.553193 & 0.230734 & 0.890298 \\ 1 & -7.553193 & 0.230733 & -0.890299 \\ 6 & -5.052717 & 0.48089 & -0.000001 \\ 8 & -5.482906 & 1.621225 & -0.000002 \\ 1 & 6.501603 & 0.679649 & -0.891698 \\ 1 & 7.258173 & -0.682971 & -0.000004 \\ 8 & 5.24602 & -0.734513 & -0.000001\end{array}$

Compound 3d

Atomic number

-0.008317
-0.006453

1.291021

1.139116

2.412976

2.276011

$-1.091284$

$-2.237887$

$-1.203189$

$-1.556825$

$-1.970362$

$-2.937222$

$-3.406350$

$-2.490878$

$-0.649140$

$-1.120929$

3.431434

$-3.648800$

$-4.472075$

0.411771

$-0.417247$

4.310081

3.406030

2.646563

3.209999

2.437310

3.209984

$-3.073429$

1.349711

$\mathbf{y}$
0.000016
0.000004
0.000009
-0.000012
-0.000007
-0.000017
0.000034
0.000040
0.000010
0.000002
0.000024
0.000015
0.000008
-0.000012
-0.000018
-0.000025
-0.000033
0.000031
0.000019
-0.000028
-0.000041
-0.000037
-0.000040
0.000012
0.891672
0.000021
-0.891662
-0.000021
0.000019

$-0.034312$

1.415686

$-0.640854$

2.114542

0.057423

1.406447

$-0.874506$

$-0.350762$

2.039655

3.401782

1.349166

3.677616

4.987038

6.040823

4.473420

5.787451

2.107833

2.856556

5.186713

4.275011

6.612801

1.615805

3.114432

$-2.591977$

$-2.307544$

$-3.661088$

$-2.307556$

7.699789

$-1.967481$

Compound $3 \mathbf{e}$

Atomic number

$-0.008832$

$-0.006859$

1.289909

1.140873

2.412754

2.275962

$-1.090908$

$-2.238188$

$\mathbf{y}$
0.000146
0.000104
0.000111
0.000039
0.000046
0.000014
0.000213
0.000245

$-0.034648$

1.416319

$-0.641904$

2.113209

0.054681

1.404321

$-0.875615$

$-0.353280$ 


$\begin{array}{lccc}7 & -1.201620 & 0.000134 & 2.041410 \\ 6 & -1.558897 & 0.000108 & 3.405126 \\ 1 & -1.969841 & 0.000185 & 1.353287 \\ 6 & -2.941462 & 0.000161 & 3.671993 \\ 6 & -3.409155 & 0.000141 & 4.982830 \\ 6 & -2.508588 & 0.000068 & 6.053039 \\ 6 & -0.652349 & 0.000035 & 4.478005 \\ 6 & -1.137941 & 0.000016 & 5.788166 \\ 7 & 3.432838 & -0.000052 & 2.104612 \\ 1 & -3.645072 & 0.000218 & 2.843665 \\ 1 & -4.479403 & 0.000182 & 5.166916 \\ 1 & -2.871397 & 0.000052 & 7.076286 \\ 1 & 0.408427 & -0.000005 & 4.279180 \\ 1 & -0.427612 & -0.000041 & 6.610243 \\ 1 & 4.310869 & -0.000071 & 1.611736 \\ 1 & 3.407536 & -0.000077 & 3.111175 \\ 6 & 2.644058 & 0.000112 & -2.593646 \\ 1 & 3.207986 & 0.891611 & -2.309420 \\ 1 & 2.435025 & 0.000148 & -3.662887 \\ 1 & 3.207916 & -0.891446 & -2.309467 \\ 8 & 1.347697 & 0.000146 & -1.969381\end{array}$

Compound $\mathbf{3 f}$

Atomic number

$\mathbf{x}$

$-1.847170$

$-0.617862$

$-3.050754$

$-0.636461$

$-3.056116$

$-1.839112$

$-1.985278$

$-0.932761$

0.545590

1.890446

0.371409

2.856470

4.212622

4.662030

2.321735

3.690652

$-1.860236$

2.534891

4.934949

1.594272

4.004414

$-2.744133$

$-0.993821$

$-5.423469$

$-5.481424$

$-6.219461$

$-5.481599$

6.658170

6.138119

6.616253

6.310676

$-4.207156$

$\underset{-0.895382}{\mathbf{y}}$

$-0.123764$

$-0.116385$

1.219660

1.204860

1.805023

$-2.258360$

$-2.953138$

$-0.803068$

$-0.378895$

$-1.819581$

$-1.403299$

$-1.099430$

0.231374

0.955292

1.238317

3.157545

$-2.441618$

$-1.912145$

1.752698

2.279469

3.639731

3.670445

$-0.003238$

0.625428

$-0.747177$

0.625987

0.063192

0.552951

0.211394

1.629662

$-0.771529$

$\mathbf{Z}$

$-0.000047$

$-0.000200$

0.000295

0.000229

0.000694

0.000760

$-0.000170$

$-0.000415$

$-0.000695$

$-0.000812$

$-0.000584$

0.001033

0.000146

$-0.002118$

$-0.004133$

$-0.004959$

0.001313

0.002154

0.000641

$-0.006932$

$-0.008492$

0.002436

0.002240

0.000584

0.892249

0.000429

$-0.890675$

$-0.826331$

0.005095

0.931215

$-0.080280$

0.000223

Compound 3g

Atomic number

$\mathbf{x}$

$-0.009500$

$-0.007619$

1.289305

1.142038

2.412025

2.275496

$-1.091966$

$-2.238996$

$-1.199541$

$-1.552988$

$-1.968411$

$-2.933476$

$-3.403378$

$-2.495381$

$-0.648664$

$-1.124099$

$\mathbf{y}$
0.000002
0.000001
0.000001
-0.000002
-0.000001
-0.000002
0.000005
0.000006
0.000002
0.000001
0.000004
0.000003
0.000002
-0.000001
-0.000003
-0.000004

$\mathbf{z}$

$-0.034375$

1.417224

$-0.641271$

2.115047

0.055291

1.405557

$-0.872809$

$-0.346681$

2.042255

3.407957

1.353787

3.686891

4.993734

6.058535

4.480004

5.793593 


$\begin{array}{lccc}7 & 3.434681 & -0.000005 & 2.103505 \\ 1 & -3.644575 & 0.000006 & 2.865163 \\ 1 & -4.467822 & 0.000005 & 5.202632 \\ 1 & 0.412766 & -0.000005 & 4.283620 \\ 1 & -0.411279 & -0.000007 & 6.615662 \\ 1 & 4.311252 & -0.000005 & 1.608148 \\ 1 & 3.412237 & -0.000005 & 3.109976 \\ 6 & 2.643951 & 0.000001 & -2.592552 \\ 1 & 3.207892 & 0.891475 & -2.308138 \\ 1 & 2.435557 & 0.000002 & -3.661949 \\ 1 & 3.207890 & -0.891475 & -2.308139 \\ 8 & -3.011523 & -0.000002 & 7.329056 \\ 1 & -2.295155 & -0.000005 & 7.977221 \\ 8 & 1.347486 & 0.000002 & -1.969020\end{array}$

Compound 3h

Atomic number

$\mathbf{x}$
-1.836983

$-0.607420$

$-3.040999$

$-0.628301$

$-3.047974$

$-1.830561$

$-1.972045$

$-0.915582$

0.554783

1.900104

0.380132

2.870743

4.227158

4.667421

2.333072

3.695446

$-1.856755$

2.555212

4.952982

1.604020

4.008907

$-2.742458$

$-0.992582$

$-5.413498$

$-5.469931$

$-6.209610$

$-5.474036$

6.290160

6.029612

6.659596

$-4.197483$

y

-0.899086
-0.125874

$-0.120686$

1.220063

1.200169

1.802163

$-2.260533$

$-2.952930$

$-0.801704$

$-0.375781$

$-1.818969$

$-1.393358$

$-1.094240$

0.240953

0.959380

1.253839

3.155709

$-2.433232$

$-1.902839$

1.755366

2.294972

3.633593

3.671117

$-0.009209$

0.624914

$-0.753110$

0.615049

1.460139

0.549323

$-0.176908$

$-0.777054$

$$
\begin{gathered}
\mathbf{z} \\
0.004090 \\
-0.001820 \\
0.003448 \\
-0.008486 \\
-0.003716 \\
-0.010405 \\
0.010500 \\
0.009965 \\
-0.000866 \\
-0.003744 \\
0.001003 \\
-0.031571 \\
-0.031213 \\
-0.001033 \\
0.024337 \\
0.025238 \\
-0.016208 \\
-0.052931 \\
-0.059216 \\
0.046441 \\
0.042283 \\
-0.043665 \\
-0.049144 \\
0.008365 \\
0.896335 \\
0.014262 \\
-0.886306 \\
0.295223 \\
-0.057142 \\
0.255014 \\
0.009782
\end{gathered}
$$

0.009782

Compound $\mathbf{3 i}$

Atomic number

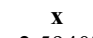

y

-0.88268
-0.15515

$-0.06024$

$-1.33686$

1.191142

1.259775

1.816569

$-2.23789$

$-2.96857$

$-0.87222$

$-0.49515$

$-1.88249$

$-1.54286$

$-1.29755$

0.024009

0.820148

1.068359

3.170459

$-2.57216$

$-2.14726$

1.645188

2.101496

3.679933

3.653583

0.138879

$-3.33361$

$-6.13488$

0.780507

$\mathbf{z}$
0.010962
-0.01337
0.018766
-0.03015
0.001988
-0.02344
0.027836
0.020125
-0.01939
-0.03813
-0.00885
-0.06743
-0.08005
-0.07402
-0.01536
-0.02802
-0.04033
-0.07499
-0.0964
0.01736
-0.00201
-0.06055
-0.08614
0.052696
0.936849 


$\begin{array}{lccc}1 & -6.95801 & -0.57477 & 0.074046 \\ 1 & -6.18416 & 0.759285 & -0.84538 \\ 7 & 5.335459 & 0.280533 & -0.12607 \\ 8 & -4.94849 & -0.67353 & 0.043834 \\ 6 & 5.811191 & 1.628268 & 0.144856 \\ 6 & 6.268531 & -0.81413 & 0.094024 \\ 1 & 6.89629 & 1.65159 & 0.03199 \\ 1 & 5.392506 & 2.342234 & -0.57225 \\ 1 & 5.560186 & 1.974894 & 1.160965 \\ 1 & 7.287633 & -0.43859 & -0.01152 \\ 1 & 6.167964 & -1.26846 & 1.093418 \\ 1 & 6.131771 & -1.6017 & -0.65443\end{array}$

\begin{tabular}{|c|c|c|c|c|c|c|c|}
\hline & & rotamer A & & & & rotamer $\mathrm{B}$ & \\
\hline Atomic number & $\mathbf{x}$ & $\mathbf{y}$ & $\mathbf{Z}$ & Atomic number & $\mathbf{x}$ & $\mathbf{y}$ & $\mathbf{Z}$ \\
\hline 6 & 2.057893 & -0.850253 & -0.000010 & 6 & 1.992585 & -0.797886 & 0.000000 \\
\hline 6 & 0.818664 & -0.104551 & -0.000013 & 6 & 0.738987 & -0.078912 & 0.000000 \\
\hline 6 & 3.268874 & -0.055923 & -0.000012 & 6 & 3.185789 & 0.018752 & 0.000000 \\
\hline 7 & 0.806009 & 1.230124 & -0.000013 & 7 & 0.689053 & 1.248290 & 0.000000 \\
\hline 7 & 3.234351 & 1.279862 & -0.000001 & 7 & 3.115438 & 1.359488 & 0.000000 \\
\hline 6 & 2.010550 & 1.842034 & -0.000008 & 6 & 1.884410 & 1.891383 & 0.000000 \\
\hline 7 & 4.461525 & -0.668935 & -0.000034 & 7 & 4.386688 & -0.568357 & 0.000000 \\
\hline 1 & 4.431394 & -1.680739 & 0.000034 & 1 & 4.352505 & -1.589779 & 0.000000 \\
\hline 7 & 2.229789 & -2.195851 & 0.000006 & 7 & 1.917923 & -2.153099 & 0.000000 \\
\hline 8 & 1.212275 & -2.948152 & 0.000008 & 8 & 2.985769 & -2.824523 & 0.000000 \\
\hline 7 & -0.329074 & -0.827121 & -0.000012 & 7 & -0.396507 & -0.831819 & 0.000000 \\
\hline 6 & -1.675664 & -0.442570 & -0.000007 & 6 & -1.753352 & -0.490950 & 0.000000 \\
\hline 1 & -0.120934 & -1.838816 & -0.000009 & 1 & -0.183099 & -1.826948 & 0.000000 \\
\hline 6 & -2.617335 & -1.492700 & -0.000009 & 6 & -2.668025 & -1.564248 & -0.000001 \\
\hline 6 & -3.978108 & -1.227458 & -0.000003 & 6 & -4.035284 & -1.334136 & -0.000001 \\
\hline 6 & -4.437227 & 0.102996 & 0.000005 & 6 & -4.529158 & -0.016486 & 0.000000 \\
\hline 6 & -2.133225 & 0.887872 & 0.000001 & 6 & -2.245207 & 0.827172 & 0.000001 \\
\hline 6 & -3.500058 & 1.149350 & 0.000007 & 6 & -3.618085 & 1.052922 & 0.000001 \\
\hline 7 & 1.983613 & 3.193204 & -0.000008 & 7 & 1.815918 & 3.241204 & -0.000001 \\
\hline 1 & -2.270154 & -2.521959 & -0.000015 & 1 & -2.297385 & -2.585749 & -0.000002 \\
\hline 1 & -4.691280 & -2.044769 & -0.000005 & 1 & -4.726643 & -2.170000 & -0.000001 \\
\hline 1 & -1.417573 & 1.695701 & 0.000003 & 1 & -1.550410 & 1.653107 & 0.000001 \\
\hline 1 & -3.847966 & 2.177131 & 0.000013 & 1 & -3.991563 & 2.071685 & 0.000002 \\
\hline 1 & 2.851227 & 3.704775 & 0.000004 & 1 & 2.667226 & 3.779521 & -0.000001 \\
\hline 1 & 1.100626 & 3.676910 & -0.000004 & 1 & 0.918395 & 3.697242 & -0.000002 \\
\hline 6 & 5.734375 & 0.034803 & 0.000047 & 6 & 5.642350 & 0.166178 & 0.000001 \\
\hline 1 & 5.832899 & 0.669525 & -0.885937 & 1 & 5.724098 & 0.803438 & -0.885804 \\
\hline 1 & 6.533655 & -0.707878 & -0.000446 & 1 & 6.458148 & -0.558068 & 0.000001 \\
\hline 1 & 5.833276 & 0.668746 & 0.886556 & 1 & 5.724098 & 0.803438 & 0.885806 \\
\hline 6 & -5.841515 & 0.386515 & 0.000011 & 6 & -5.940091 & 0.230484 & 0.000000 \\
\hline 7 & -6.982887 & 0.618132 & 0.000017 & 7 & -7.087234 & 0.431815 & 0.000000 \\
\hline
\end{tabular}

Compound $\mathbf{4 b}$

\begin{tabular}{|c|c|c|c|c|c|c|c|}
\hline & & rotamer A & & & & rotamer B & $\mathbf{z}$ \\
\hline Atomic number & $\mathbf{x}$ & $\mathbf{y}$ & $\mathbf{z}$ & Atomic number & $\mathbf{x}$ & $\mathbf{y}$ & $\mathbf{z}$ \\
\hline 6 & 2.855026 & -0.846090 & -0.000007 & 6 & 2.795834 & -0.797722 & -0.000335 \\
\hline 6 & 1.601620 & -0.123134 & -0.000022 & 6 & 1.530935 & -0.096916 & -0.007015 \\
\hline 6 & 4.051202 & -0.029942 & 0.000012 & 6 & 3.976981 & 0.036174 & 0.005898 \\
\hline 7 & 1.565924 & 1.211915 & -0.000017 & 7 & 1.463427 & 1.230236 & -0.007634 \\
\hline 7 & 3.993114 & 1.304853 & 0.000018 & 7 & 3.888036 & 1.375701 & 0.005318 \\
\hline 6 & 2.758718 & 1.844610 & 0.000005 & 6 & 2.648966 & 1.889544 & -0.001314 \\
\hline 7 & 5.254887 & -0.621853 & 0.000025 & 7 & 5.186218 & -0.534125 & 0.012493 \\
\hline 1 & 5.242227 & -1.634009 & 0.000022 & 1 & 5.165770 & -1.556082 & 0.012482 \\
\hline 7 & 3.051853 & -2.187957 & -0.000007 & 7 & 2.741685 & -2.153389 & -0.000384 \\
\hline 8 & 2.048388 & -2.959273 & -0.000021 & 8 & 3.819791 & -2.809339 & 0.005406 \\
\hline 7 & 0.468034 & -0.864763 & -0.000037 & 7 & 0.407121 & -0.864084 & -0.012429 \\
\hline 6 & -0.887452 & -0.503250 & -0.000044 & 6 & -0.956619 & -0.540906 & -0.018962 \\
\hline 1 & 0.692629 & -1.872417 & -0.000031 & 1 & 0.632630 & -1.856318 & -0.009740 \\
\hline 6 & -1.809052 & -1.571007 & 0.000004 & 6 & -1.855976 & -1.625243 & -0.016708 \\
\hline 6 & -3.174323 & -1.327777 & 0.000000 & 6 & -3.228015 & -1.411002 & -0.024421 \\
\hline 6 & -3.653152 & -0.010219 & -0.000055 & 6 & -3.733221 & -0.105567 & -0.037446 \\
\hline 6 & -1.370068 & 0.815523 & -0.000100 & 6 & -1.466499 & 0.768902 & -0.030898 \\
\hline 6 & -2.745919 & 1.050048 & -0.000103 & 6 & -2.844943 & 0.973324 & -0.038521 \\
\hline 7 & 2.708219 & 3.195516 & 0.000014 & 7 & 2.561761 & 3.238658 & -0.002027 \\
\hline 1 & -1.443296 & -2.593824 & 0.000043 & 1 & -1.472462 & -2.642012 & -0.011354 \\
\hline
\end{tabular}




\begin{tabular}{|c|c|c|c|c|c|c|c|}
\hline 1 & -3.869261 & -2.161365 & 0.000037 & 1 & -3.904758 & -2.258506 & -0.026671 \\
\hline 1 & -0.671312 & 1.637970 & -0.000141 & 1 & -0.784241 & 1.605130 & -0.036289 \\
\hline 1 & -3.108868 & 2.071813 & -0.000150 & 1 & -3.228667 & 1.987980 & -0.051682 \\
\hline 1 & 3.566728 & 3.722095 & 0.000044 & 1 & 3.405443 & 3.788694 & 0.004659 \\
\hline 1 & 1.816668 & 3.663186 & 0.000022 & 1 & 1.657757 & 3.681651 & -0.004286 \\
\hline 6 & 6.514847 & 0.104223 & 0.000050 & 6 & 6.431135 & 0.218038 & 0.019316 \\
\hline 1 & 6.602566 & 0.740346 & -0.886139 & 1 & 6.509079 & 0.856263 & -0.866202 \\
\hline 1 & 7.327249 & -0.624160 & 0.000052 & 1 & 7.257199 & -0.494534 & 0.024233 \\
\hline 1 & 6.602543 & 0.740327 & 0.886254 & 1 & 6.498995 & 0.856817 & 0.905262 \\
\hline 6 & -5.136112 & 0.225366 & 0.000021 & 6 & -5.214643 & 0.132858 & 0.008968 \\
\hline 9 & -5.458911 & 1.541311 & -0.000519 & 9 & -5.571476 & 1.289435 & -0.606128 \\
\hline 9 & -5.741031 & -0.328494 & 1.087493 & 9 & -5.681419 & 0.222369 & 1.287951 \\
\hline 9 & -5.741302 & -0.329469 & -1.086794 & 9 & -5.917819 & -0.866720 & -0.582089 \\
\hline
\end{tabular}

Compound 4c

\begin{tabular}{|c|c|c|c|c|c|c|c|}
\hline & & rotamer $\mathrm{A}$ & & & & rotamer B & \\
\hline Atomic number & $\mathbf{x}$ & $\mathbf{y}$ & $\mathbf{Z}$ & Atomic number & $\mathbf{x}$ & $\mathbf{y}$ & $\mathbf{Z}$ \\
\hline 6 & -2.874262 & -0.864670 & -0.000017 & 6 & 2.811405 & -0.817232 & -0.000001 \\
\hline 6 & -1.637282 & -0.113204 & -0.000021 & 6 & 1.566838 & -0.079555 & -0.000039 \\
\hline 6 & -4.088729 & -0.075783 & -0.000017 & 6 & 4.016318 & -0.017658 & 0.000011 \\
\hline 7 & -1.633382 & 1.222504 & -0.000026 & 7 & 1.539131 & 1.249134 & -0.000050 \\
\hline 7 & -4.061896 & 1.259760 & 0.000004 & 7 & 3.967025 & 1.323683 & -0.000007 \\
\hline 6 & -2.839472 & 1.827490 & -0.000018 & 6 & 2.742727 & 1.873217 & -0.000033 \\
\hline 7 & -5.278811 & -0.695527 & -0.000059 & 7 & 5.208439 & -0.623547 & 0.000053 \\
\hline 1 & -5.242546 & -1.707077 & 0.000040 & 1 & 5.157156 & -1.644556 & 0.000083 \\
\hline 7 & -3.041579 & -2.210425 & 0.000007 & 7 & 2.718949 & -2.170447 & 0.000006 \\
\hline 8 & -2.021433 & -2.959771 & 0.000010 & 8 & 3.778461 & -2.857171 & 0.000033 \\
\hline 7 & -0.486484 & -0.827444 & -0.000019 & 7 & 0.421247 & -0.812766 & -0.000057 \\
\hline 6 & 0.860688 & -0.436156 & -0.000011 & 6 & -0.933148 & -0.452397 & -0.000062 \\
\hline 1 & -0.686423 & -1.839812 & -0.000017 & 1 & 0.617375 & -1.811174 & -0.000052 \\
\hline 6 & 1.805029 & -1.483330 & -0.000035 & 6 & -1.860175 & -1.514511 & -0.000071 \\
\hline 6 & 3.165867 & -1.211458 & -0.000026 & 6 & -3.225470 & -1.265524 & -0.000047 \\
\hline 6 & 3.622226 & 0.116996 & 0.000005 & 6 & -3.704442 & 0.054651 & -0.000002 \\
\hline 6 & 1.313972 & 0.895686 & 0.000023 & 6 & -1.408610 & 0.871574 & -0.000036 \\
\hline 6 & 2.681435 & 1.155632 & 0.000029 & 6 & -2.780044 & 1.108170 & -0.000001 \\
\hline 7 & -2.819791 & 3.179376 & -0.000028 & 7 & 2.694739 & 3.224391 & -0.000057 \\
\hline 1 & 1.459329 & -2.513427 & -0.000061 & 1 & -1.499941 & -2.540137 & -0.000097 \\
\hline 1 & 3.880063 & -2.026559 & -0.000044 & 1 & -3.925588 & -2.092765 & -0.000056 \\
\hline 1 & 0.596843 & 1.702107 & 0.000046 & 1 & -0.704908 & 1.689860 & -0.000040 \\
\hline 1 & 3.039231 & 2.179932 & 0.000054 & 1 & -3.154268 & 2.126596 & 0.000027 \\
\hline 1 & -3.689833 & 3.686538 & -0.000056 & 1 & 3.553797 & 3.750015 & 0.000028 \\
\hline 1 & -1.938662 & 3.666566 & -0.000081 & 1 & 1.803487 & 3.692715 & 0.000001 \\
\hline 6 & -6.554766 & 0.001581 & 0.000100 & 6 & 6.474642 & 0.091778 & 0.000087 \\
\hline 1 & -6.656803 & 0.636318 & -0.885614 & 1 & 6.566308 & 0.728035 & 0.885771 \\
\hline 8 & 5.868760 & -0.622449 & 0.000016 & 8 & -5.937889 & -0.722555 & -0.000006 \\
\hline 6 & 7.282960 & -0.357933 & 0.000017 & 6 & -7.356263 & -0.481433 & 0.000038 \\
\hline 1 & 7.760527 & -1.337553 & 0.000035 & 1 & -7.817637 & -1.468799 & -0.000012 \\
\hline 1 & 7.565203 & 0.209416 & -0.890224 & 1 & -7.647881 & 0.081125 & 0.890288 \\
\hline 1 & 7.565197 & 0.209448 & 0.890238 & 1 & -7.647918 & 0.081230 & -0.890134 \\
\hline 6 & 5.065581 & 0.469167 & 0.000011 & 6 & -5.152821 & 0.382575 & 0.000042 \\
\hline 8 & 5.501407 & 1.607489 & 0.000011 & 8 & -5.607874 & 1.513395 & 0.000117 \\
\hline 1 & -6.657315 & 0.635137 & 0.886614 & 1 & 6.566353 & 0.728037 & -0.885589 \\
\hline 1 & -7.350551 & -0.745002 & -0.000626 & 1 & 7.279642 & -0.644598 & 0.000108 \\
\hline
\end{tabular}

Compound 4d

\begin{tabular}{|c|c|c|c|c|c|c|c|}
\hline & & rotamer A & & & & rotamer B & \\
\hline Atomic number & $\mathbf{x}$ & $\mathbf{y}$ & $\mathbf{z}$ & Atomic number & $\mathbf{x}$ & $\mathbf{y}$ & $\mathbf{z}$ \\
\hline 6 & 2.205213 & -0.853813 & -0.000005 & 6 & -2.141882 & -0.801737 & -0.000001 \\
\hline 6 & 0.961031 & -0.113014 & -0.000005 & 6 & -0.883389 & -0.086076 & -0.000002 \\
\hline 6 & 3.412703 & -0.054598 & -0.000008 & 6 & -3.332278 & 0.018862 & 0.000000 \\
\hline 7 & 0.946359 & 1.224535 & -0.000006 & 7 & -0.833529 & 1.243701 & -0.000003 \\
\hline 7 & 3.374652 & 1.280530 & 0.000002 & 7 & -3.259950 & 1.359184 & -0.000001 \\
\hline 6 & 2.147046 & 1.838126 & -0.000003 & 6 & -2.025977 & 1.887244 & -0.000004 \\
\hline 7 & 4.607941 & -0.664601 & -0.000027 & 7 & -4.534605 & -0.566716 & 0.000003 \\
\hline 1 & 4.579629 & -1.676416 & 0.000019 & 1 & -4.499970 & -1.588624 & 0.000003 \\
\hline 7 & 2.382428 & -2.197344 & 0.000004 & 7 & -2.073563 & -2.155650 & 0.000001 \\
\hline 8 & 1.366472 & -2.954042 & 0.000006 & 8 & -3.145242 & -2.824407 & 0.000002 \\
\hline 7 & -0.181431 & -0.835071 & -0.000005 & 7 & 0.247549 & -0.836144 & -0.000001 \\
\hline 6 & -1.534286 & -0.451380 & -0.000003 & 6 & 1.610950 & -0.493959 & -0.000001 \\
\hline 1 & 0.026878 & -1.846070 & -0.000003 & 1 & 0.036486 & -1.831296 & -0.000001 \\
\hline 6 & -2.473450 & -1.500039 & -0.000002 & 6 & 2.524566 & -1.564186 & -0.000009 \\
\hline 6 & -3.839555 & -1.238283 & 0.000001 & 6 & 3.897046 & -1.335537 & -0.000008 \\
\hline
\end{tabular}




\begin{tabular}{|c|c|c|c|c|c|c|c|}
\hline 6 & -4.281604 & 0.085736 & 0.000002 & 6 & 4.371560 & -0.023239 & 0.000001 \\
\hline 6 & -1.995826 & 0.875209 & -0.000002 & 6 & 2.104467 & 0.820814 & 0.000009 \\
\hline 6 & -3.367202 & 1.136813 & 0.000000 & 6 & 3.481797 & 1.048805 & 0.000010 \\
\hline 7 & 2.118800 & 3.190533 & -0.000002 & 7 & -1.956763 & 3.238137 & -0.000007 \\
\hline 1 & -2.127806 & -2.530102 & -0.000002 & 1 & 2.157079 & -2.587138 & -0.000017 \\
\hline 1 & -4.553338 & -2.054478 & 0.000001 & 1 & 4.590307 & -2.169259 & -0.000015 \\
\hline 1 & -1.284231 & 1.686991 & -0.000003 & 1 & 1.412578 & 1.649548 & 0.000016 \\
\hline 1 & -3.720271 & 2.162343 & 0.000001 & 1 & 3.858770 & 2.065802 & 0.000018 \\
\hline 1 & 2.985838 & 3.702708 & 0.000012 & 1 & -2.807736 & 3.776666 & -0.000014 \\
\hline 1 & 1.234978 & 3.672461 & 0.000008 & 1 & -1.058631 & 3.692746 & -0.000017 \\
\hline 6 & 5.878065 & 0.042802 & 0.000023 & 6 & -5.788490 & 0.169750 & 0.000006 \\
\hline 1 & 5.975182 & 0.678099 & -0.885882 & 1 & -5.869606 & 0.807519 & 0.885667 \\
\hline 1 & 6.679951 & -0.697253 & -0.000346 & 1 & -6.605819 & -0.552956 & 0.000001 \\
\hline 1 & 5.975476 & 0.677527 & 0.886312 & 1 & -5.869604 & 0.807529 & -0.885648 \\
\hline 17 & -6.006948 & 0.427123 & 0.000004 & 17 & 6.104935 & 0.276629 & 0.000002 \\
\hline
\end{tabular}

Compound 4e

\begin{tabular}{|c|c|c|c|c|c|c|c|}
\hline & & rotamer A & & & & rotamer B & \\
\hline Atomic number & $\mathbf{x}$ & $\mathbf{y}$ & $\mathbf{z}$ & Atomic number & $\mathbf{x}$ & $\mathbf{y}$ & $\mathbf{z}$ \\
\hline 6 & 1.401737 & -0.879275 & 0.000049 & 6 & 1.345103 & -0.814502 & -0.000010 \\
\hline 6 & 0.201613 & -0.067436 & 0.000054 & 6 & 0.113127 & -0.051553 & -0.000009 \\
\hline 6 & 2.653650 & -0.151882 & 0.000013 & 6 & 2.565679 & -0.039325 & -0.000004 \\
\hline 7 & 0.266877 & 1.269480 & 0.000052 & 7 & 0.115902 & 1.279952 & -0.000007 \\
\hline 7 & 2.694401 & 1.182963 & 0.000044 & 7 & 2.545116 & 1.302589 & -0.000010 \\
\hline 6 & 1.500432 & 1.810854 & 0.000053 & 6 & 1.331111 & 1.876522 & -0.000009 \\
\hline 7 & 3.811548 & -0.831259 & -0.000079 & 7 & 3.744994 & -0.670744 & 0.000012 \\
\hline 1 & 3.723498 & -1.839625 & -0.000056 & 1 & 3.670257 & -1.690755 & 0.000008 \\
\hline 7 & 1.502339 & -2.230521 & 0.000054 & 7 & 1.227252 & -2.164485 & -0.000013 \\
\hline 8 & 0.445463 & -2.928942 & 0.000080 & 8 & 2.274087 & -2.872978 & -0.000013 \\
\hline 7 & -0.980353 & -0.720228 & 0.000050 & 7 & -1.044341 & -0.756753 & -0.000009 \\
\hline 6 & -2.312310 & -0.261697 & 0.000010 & 6 & -2.397423 & -0.367224 & -0.000002 \\
\hline 1 & -0.833043 & -1.741117 & 0.000061 & 1 & -0.871737 & -1.759171 & -0.000010 \\
\hline 6 & -3.303906 & -1.261556 & -0.000009 & 6 & -3.343057 & -1.409897 & 0.000007 \\
\hline 6 & -4.653150 & -0.919917 & -0.000052 & 6 & -4.706742 & -1.129472 & 0.000014 \\
\hline 6 & -5.040707 & 0.424112 & -0.000079 & 6 & -5.155128 & 0.195099 & 0.000013 \\
\hline 6 & -2.698713 & 1.088914 & -0.000015 & 6 & -2.844429 & 0.964209 & -0.000003 \\
\hline 6 & -4.057314 & 1.415104 & -0.000060 & 6 & -4.216364 & 1.228497 & 0.000004 \\
\hline 7 & 1.552152 & 3.163204 & 0.000063 & 7 & 1.313917 & 3.229618 & -0.000011 \\
\hline 1 & -3.007341 & -2.307169 & 0.000011 & 1 & -3.002454 & -2.442556 & 0.000008 \\
\hline 1 & -5.402165 & -1.706317 & -0.000066 & 1 & -5.418863 & -1.949467 & 0.000020 \\
\hline 1 & -6.092709 & 0.692500 & -0.000115 & 1 & -6.218043 & 0.415745 & 0.000019 \\
\hline 1 & -1.941586 & 1.858305 & 0.000000 & 1 & -2.123059 & 1.767341 & -0.000011 \\
\hline 1 & -4.344635 & 2.462918 & -0.000082 & 1 & -4.549690 & 2.262588 & 0.000003 \\
\hline 1 & 2.447763 & 3.623356 & 0.000049 & 1 & 2.184737 & 3.735227 & 0.000009 \\
\hline 1 & 0.697785 & 3.695553 & 0.000050 & 1 & 0.433356 & 3.717363 & 0.000013 \\
\hline 6 & 5.120610 & -0.199649 & -0.000163 & 6 & 5.025682 & 0.017378 & 0.000033 \\
\hline 1 & 5.254823 & 0.429281 & -0.885864 & 1 & 5.131109 & 0.652056 & -0.885385 \\
\hline 1 & 5.877975 & -0.985323 & -0.000905 & 1 & 5.815132 & -0.735787 & -0.000240 \\
\hline 1 & 5.255533 & 0.428249 & 0.886180 & 1 & 5.131309 & 0.651615 & 0.885744 \\
\hline
\end{tabular}

Compound 4f

\begin{tabular}{|c|c|c|c|c|c|c|c|}
\hline & & rotamer A & & & & rotamer B & \\
\hline Atomic number & $\mathbf{x}$ & $\mathbf{y}$ & $\mathbf{z}$ & Atomic number & $\mathbf{x}$ & $\mathbf{y}$ & $\mathbf{z}$ \\
\hline 6 & -1.833103 & -0.863961 & -0.000032 & 6 & -1.770496 & -0.807335 & 0.000047 \\
\hline 6 & -0.602871 & -0.097929 & -0.000208 & 6 & -0.519921 & -0.074133 & 0.000016 \\
\hline 6 & -3.056492 & -0.089547 & 0.000312 & 6 & -2.971870 & -0.002640 & 0.000240 \\
\hline 7 & -0.618094 & 1.241338 & 0.000200 & 7 & -0.491169 & 1.257828 & 0.000313 \\
\hline 7 & -3.047136 & 1.245691 & 0.000714 & 7 & -2.919243 & 1.338280 & 0.000571 \\
\hline 6 & -1.829836 & 1.828157 & 0.000744 & 6 & -1.691312 & 1.882774 & 0.000659 \\
\hline 7 & -4.239296 & -0.725043 & 0.000263 & 7 & -4.166036 & -0.605863 & 0.000102 \\
\hline 1 & -4.188836 & -1.735962 & 0.000043 & 1 & -4.115163 & -1.627479 & -0.000088 \\
\hline 7 & -1.984372 & -2.210106 & -0.000139 & 7 & -1.685776 & -2.159330 & -0.000165 \\
\hline 8 & -0.953604 & -2.947477 & -0.000384 & 8 & -2.749857 & -2.842629 & -0.000218 \\
\hline 7 & 0.553299 & -0.793138 & -0.000708 & 7 & 0.619913 & -0.804996 & -0.000255 \\
\hline 6 & 1.901043 & -0.381739 & -0.000806 & 6 & 1.981953 & -0.444435 & -0.000421 \\
\hline 1 & 0.368952 & -1.807974 & -0.000552 & 1 & 0.425461 & -1.803317 & -0.000121 \\
\hline 6 & 2.860414 & -1.412458 & 0.000933 & 6 & 2.908166 & -1.504129 & 0.000848 \\
\hline 6 & 4.218546 & -1.117164 & -0.000029 & 6 & 4.275440 & -1.252110 & -0.000225 \\
\hline 6 & 4.676563 & 0.210727 & -0.002328 & 6 & 4.775757 & 0.059923 & -0.002105 \\
\hline 6 & 2.340992 & 0.949839 & -0.004217 & 6 & 2.463746 & 0.872111 & -0.003183 \\
\hline 6 & 3.711681 & 1.224006 & -0.005136 & 6 & 3.842585 & 1.102376 & -0.004164 \\
\hline 7 & -1.831463 & 3.181845 & 0.001237 & 7 & -1.642437 & 3.235405 & 0.001021 \\
\hline
\end{tabular}




\begin{tabular}{|c|c|c|c|c|c|c|c|}
\hline 1 & 2.531892 & -2.448621 & 0.001994 & 1 & 2.549847 & -2.530914 & 0.001659 \\
\hline 1 & 4.935792 & -1.934446 & 0.000343 & 1 & 4.965740 & -2.092290 & -0.000089 \\
\hline 1 & 1.617274 & 1.750919 & -0.007058 & 1 & 1.765729 & 1.695811 & -0.005409 \\
\hline 1 & 4.032252 & 2.263132 & -0.008786 & 1 & 4.195120 & 2.131109 & -0.007166 \\
\hline 1 & -2.709356 & 3.674863 & 0.002625 & 1 & -2.501177 & 3.761196 & 0.002118 \\
\hline 1 & -0.957837 & 3.681893 & 0.002421 & 1 & -0.750595 & 3.702140 & 0.002086 \\
\hline 6 & -5.523437 & -0.044493 & 0.000658 & 6 & -5.429842 & 0.112542 & 0.000209 \\
\hline 1 & -5.634304 & 0.588273 & 0.886920 & 1 & -5.520443 & 0.749170 & 0.885912 \\
\hline 1 & -6.309990 & -0.800979 & 0.000368 & 1 & -6.237069 & -0.621582 & -0.000062 \\
\hline 1 & -5.634405 & 0.589058 & -0.885025 & 1 & -5.520296 & 0.749664 & -0.885153 \\
\hline 1 & 6.673448 & 0.024072 & -0.821532 & 1 & 6.763228 & -0.176886 & -0.832378 \\
\hline 6 & 6.154792 & 0.522735 & 0.005496 & 6 & 6.263269 & 0.325125 & 0.004165 \\
\hline 1 & 6.629206 & 0.184685 & 0.934899 & 1 & 6.730862 & -0.040538 & 0.926491 \\
\hline 1 & 6.334417 & 1.597760 & -0.086869 & 1 & 6.476001 & 1.395262 & -0.073690 \\
\hline
\end{tabular}

Compound 4g

\begin{tabular}{|c|c|c|c|c|c|c|c|}
\hline & & rotamer $\mathrm{A}$ & & & & rotamer B & \\
\hline Atomic number & $\mathbf{x}$ & $\mathbf{y}$ & $\mathbf{z}$ & Atomic number & $\mathbf{x}$ & $\mathbf{y}$ & $\mathbf{z}$ \\
\hline 6 & 1.813876 & -0.866627 & -0.000001 & 6 & -1.752784 & -0.809104 & -0.000001 \\
\hline 6 & 0.584223 & -0.099581 & -0.000003 & 6 & -0.501272 & -0.077010 & -0.000011 \\
\hline 6 & 3.037816 & -0.093250 & -0.000001 & 6 & -2.953265 & -0.003359 & -0.000001 \\
\hline 7 & 0.600335 & 1.241024 & -0.000004 & 7 & -0.471438 & 1.256122 & -0.000025 \\
\hline 7 & 3.029423 & 1.241947 & -0.000002 & 7 & -2.899483 & 1.337582 & -0.000015 \\
\hline 6 & 1.812570 & 1.825882 & -0.000004 & 6 & -1.671132 & 1.881276 & -0.000031 \\
\hline 7 & 4.219901 & -0.729965 & -0.000002 & 7 & -4.147826 & -0.605677 & 0.000011 \\
\hline 1 & 4.168227 & -1.740851 & 0.000006 & 1 & -4.097474 & -1.627418 & 0.000039 \\
\hline 7 & 1.962421 & -2.212465 & 0.000001 & 7 & -1.669150 & -2.160834 & 0.000015 \\
\hline 8 & 0.928860 & -2.947249 & 0.000001 & 8 & -2.733802 & -2.843676 & 0.000031 \\
\hline 7 & -0.571294 & -0.793167 & -0.000002 & 7 & 0.637096 & -0.807323 & -0.000007 \\
\hline 6 & -1.918977 & -0.379449 & -0.000001 & 6 & 2.000176 & -0.445312 & -0.000004 \\
\hline 1 & -0.386619 & -1.808639 & -0.000002 & 1 & 0.442718 & -1.805665 & -0.000006 \\
\hline 6 & -2.882380 & -1.406769 & -0.000007 & 6 & 2.929730 & -1.502085 & -0.000076 \\
\hline 6 & -4.241097 & -1.118646 & -0.000005 & 6 & 4.297698 & -1.257818 & -0.000067 \\
\hline 6 & -4.668841 & 0.213685 & 0.000002 & 6 & 4.768700 & 0.059248 & 0.000012 \\
\hline 6 & -2.354923 & 0.953805 & 0.000006 & 6 & 2.478646 & 0.872630 & 0.000076 \\
\hline 6 & -3.722179 & 1.240488 & 0.000008 & 6 & 3.854779 & 1.114925 & 0.000082 \\
\hline 7 & 1.817574 & 3.179989 & -0.000006 & 7 & -1.622926 & 3.234313 & -0.000050 \\
\hline 1 & -2.557396 & -2.443745 & -0.000012 & 1 & 2.574673 & -2.529666 & -0.000139 \\
\hline 1 & -4.978890 & -1.913898 & -0.000009 & 1 & 5.008789 & -2.077055 & -0.000122 \\
\hline 1 & -1.628072 & 1.752138 & 0.000011 & 1 & 1.777952 & 1.694111 & 0.000131 \\
\hline 1 & -4.046345 & 2.279201 & 0.000014 & 1 & 4.210896 & 2.143060 & 0.000145 \\
\hline 1 & 2.696946 & 3.670340 & -0.000008 & 1 & -2.482214 & 3.759192 & -0.000081 \\
\hline 1 & 0.945697 & 3.682835 & -0.000010 & 1 & -0.731790 & 3.702126 & -0.000096 \\
\hline 6 & 5.504840 & -0.050965 & 0.000010 & 6 & -5.411127 & 0.113589 & 0.000032 \\
\hline 1 & 5.616494 & 0.582092 & -0.885945 & 1 & -5.501292 & 0.750442 & 0.885623 \\
\hline 1 & 6.290503 & -0.808372 & -0.000043 & 1 & -6.218856 & -0.619977 & -0.000065 \\
\hline 1 & 5.616530 & 0.582007 & 0.886022 & 1 & -5.501208 & 0.750601 & -0.885449 \\
\hline 8 & -6.021410 & 0.443504 & 0.000003 & 8 & 6.128780 & 0.244931 & 0.000016 \\
\hline 1 & -6.194741 & 1.393878 & 0.000009 & 1 & 6.333179 & 1.189053 & 0.000080 \\
\hline
\end{tabular}

Compound 4h

\begin{tabular}{|c|c|c|c|c|c|c|c|}
\hline & & rotamer A & & & & rotamer B & \\
\hline Atomic number & $\mathbf{x}$ & $\mathbf{y}$ & $\mathbf{z}$ & Atomic number & $\mathbf{x}$ & $\mathbf{y}$ & $\mathbf{z}$ \\
\hline 6 & -1.823099 & -0.866936 & 0.003653 & 6 & -1.761986 & -0.809780 & 0.003746 \\
\hline 6 & -0.592568 & -0.100076 & -0.001862 & 6 & -0.509216 & -0.077598 & -0.002397 \\
\hline 6 & -3.046634 & -0.092628 & 0.003371 & 6 & -2.962107 & -0.003035 & 0.002508 \\
\hline 7 & -0.609497 & 1.241799 & -0.007519 & 7 & -0.480596 & 1.256641 & -0.008956 \\
\hline 7 & -3.038481 & 1.242113 & -0.003153 & 7 & -2.908688 & 1.337566 & -0.005615 \\
\hline 6 & -1.820387 & 1.825868 & -0.008911 & 6 & -1.679221 & 1.880877 & -0.011577 \\
\hline 7 & -4.229023 & -0.729834 & 0.009018 & 7 & -4.156631 & -0.606093 & 0.008797 \\
\hline 1 & -4.176559 & -1.740651 & 0.012573 & 1 & -4.104698 & -1.628019 & 0.013544 \\
\hline 7 & -1.972336 & -2.211897 & 0.009090 & 7 & -1.680183 & -2.160716 & 0.009962 \\
\hline 8 & -0.937863 & -2.947377 & 0.008671 & 8 & -2.746208 & -2.843508 & 0.015817 \\
\hline 7 & 0.562165 & -0.792209 & -0.001479 & 7 & 0.628238 & -0.805976 & -0.001446 \\
\hline 6 & 1.910578 & -0.379253 & -0.004215 & 6 & 1.992632 & -0.444412 & -0.003950 \\
\hline 1 & 0.377331 & -1.807701 & 0.000089 & 1 & 0.434565 & -1.804430 & -0.000965 \\
\hline 6 & 2.874448 & -1.403306 & -0.028980 & 6 & 2.922676 & -1.497455 & -0.037137 \\
\hline 6 & 4.232930 & -1.112771 & -0.028415 & 6 & 4.290729 & -1.250401 & -0.035879 \\
\hline 6 & 4.681725 & 0.219514 & -0.001124 & 6 & 4.782244 & 0.065639 & -0.000204 \\
\hline 6 & 2.352195 & 0.953302 & 0.020752 & 6 & 2.476168 & 0.872476 & 0.029811 \\
\hline 6 & 3.716533 & 1.238780 & 0.021860 & 6 & 3.849434 & 1.113861 & 0.030933 \\
\hline 7 & -1.826625 & 3.180739 & -0.012047 & 7 & -1.631946 & 3.234558 & -0.016467 \\
\hline
\end{tabular}




\begin{tabular}{|c|c|c|c|c|c|c|c|}
\hline 1 & 2.551871 & -2.441079 & -0.048014 & 1 & 2.570195 & -2.525843 & -0.063398 \\
\hline 1 & 4.953712 & -1.926032 & -0.054055 & 1 & 4.984295 & -2.086770 & -0.068328 \\
\hline 1 & 1.626912 & 1.753050 & 0.040479 & 1 & 1.776906 & 1.694993 & 0.055765 \\
\hline 1 & 4.036881 & 2.277898 & 0.036522 & 1 & 4.201724 & 2.142451 & 0.052097 \\
\hline 1 & -2.706156 & 3.669385 & -0.047924 & 1 & -2.491385 & 3.757870 & -0.052679 \\
\hline 1 & -0.955198 & 3.682701 & -0.054049 & 1 & -0.741194 & 3.701280 & -0.059256 \\
\hline 6 & -5.513672 & -0.050977 & 0.008238 & 6 & -5.419888 & 0.112680 & 0.007844 \\
\hline 1 & -5.623289 & 0.587416 & 0.890687 & 1 & -5.507639 & 0.755616 & 0.889338 \\
\hline 1 & -6.299527 & -0.808262 & 0.014643 & 1 & -6.227589 & -0.620983 & 0.015093 \\
\hline 1 & -5.627882 & 0.577092 & -0.881060 & 1 & -5.513103 & 0.743967 & -0.881513 \\
\hline 1 & 6.311402 & 1.428540 & 0.295615 & 1 & 6.448720 & 1.219400 & 0.307069 \\
\hline 7 & 6.046797 & 0.518958 & -0.057188 & 7 & 6.157644 & 0.322436 & -0.057508 \\
\hline 1 & 6.670118 & -0.210143 & 0.261869 & 1 & 6.755206 & -0.427478 & 0.263139 \\
\hline
\end{tabular}

Compound 5a

\begin{tabular}{|c|c|c|c|c|c|c|c|}
\hline & & rotamer $\mathrm{A}$ & & & & rotamer B & \\
\hline Atomic number & $\mathbf{x}$ & $\mathbf{y}$ & $\mathbf{z}$ & Atomic number & $\mathbf{x}$ & $\mathbf{y}$ & $\mathbf{z}$ \\
\hline 6 & -1.444521 & -0.826707 & -0.120597 & 6 & -1.382129 & -0.789054 & -0.116037 \\
\hline 6 & -0.199888 & -0.090284 & -0.081087 & 6 & -0.126211 & -0.073858 & -0.084562 \\
\hline 6 & -2.648671 & -0.023802 & -0.165683 & 6 & -2.571738 & 0.031070 & -0.163233 \\
\hline 7 & -0.177221 & 1.243893 & -0.091929 & 7 & -0.071564 & 1.252740 & -0.100011 \\
\hline 7 & -2.603984 & 1.309921 & -0.184565 & 7 & -2.496451 & 1.370101 & -0.186683 \\
\hline 6 & -1.376838 & 1.863320 & -0.145735 & 6 & -1.264616 & 1.898330 & -0.152659 \\
\hline 7 & -3.846050 & -0.637405 & -0.193085 & 7 & -3.775263 & -0.558992 & -0.193106 \\
\hline 1 & -3.812417 & -1.649304 & -0.243533 & 1 & -3.738681 & -1.581294 & -0.212840 \\
\hline 7 & -1.624701 & -2.171704 & -0.130623 & 7 & -1.308566 & -2.144927 & -0.104278 \\
\hline 8 & -0.612947 & -2.930305 & -0.089192 & 8 & -2.375541 & -2.816767 & -0.130796 \\
\hline 7 & 0.941803 & -0.821009 & -0.034886 & 7 & 1.006192 & -0.830194 & -0.038032 \\
\hline 6 & 2.290481 & -0.445822 & 0.003561 & 6 & 2.363342 & -0.493019 & 0.000532 \\
\hline 1 & 0.726582 & -1.831131 & -0.036653 & 1 & 0.789610 & -1.824632 & -0.036090 \\
\hline 6 & 3.224201 & -1.502453 & 0.038875 & 6 & 3.274665 & -1.568788 & 0.030510 \\
\hline 6 & 4.586205 & -1.246709 & 0.078211 & 6 & 4.641920 & -1.342431 & 0.071546 \\
\hline 6 & 5.054672 & 0.080478 & 0.083461 & 6 & 5.139281 & -0.026136 & 0.083916 \\
\hline 6 & 2.757393 & 0.881372 & 0.009179 & 6 & 2.858736 & 0.823769 & 0.012741 \\
\hline 6 & 4.125449 & 1.133308 & 0.048626 & 6 & 4.231608 & 1.045740 & 0.054030 \\
\hline 7 & -1.341194 & 3.214131 & -0.163270 & 7 & -1.192710 & 3.247864 & -0.173420 \\
\hline 1 & 2.869909 & -2.529271 & 0.034852 & 1 & 2.901364 & -2.589274 & 0.021208 \\
\hline 1 & 5.293175 & -2.068964 & 0.104858 & 1 & 5.330618 & -2.180189 & 0.094092 \\
\hline 1 & 2.047875 & 1.694158 & -0.017589 & 1 & 2.166594 & 1.651610 & -0.010250 \\
\hline 1 & 4.480533 & 2.158623 & 0.052491 & 1 & 4.607745 & 2.063485 & 0.063464 \\
\hline 1 & -2.205792 & 3.729727 & -0.197782 & 1 & -2.043003 & 3.786910 & -0.205138 \\
\hline 1 & -0.456129 & 3.693193 & -0.135108 & 1 & -0.294978 & 3.702663 & -0.146364 \\
\hline 6 & -5.102900 & 0.046521 & -0.323931 & 6 & -5.017598 & 0.157048 & -0.290073 \\
\hline 6 & -5.800841 & 0.572696 & 0.906293 & 6 & -5.733252 & 0.593439 & 0.966821 \\
\hline 6 & -6.328983 & -0.653418 & 0.196015 & 6 & -6.266590 & -0.558323 & 0.143727 \\
\hline 1 & -5.197648 & 0.633226 & -1.234523 & 1 & -5.075721 & 0.821792 & -1.147547 \\
\hline 1 & -5.299595 & 0.426749 & 1.857630 & 1 & -5.257559 & 0.357620 & 1.913443 \\
\hline 1 & -6.345892 & 1.507062 & 0.814509 & 1 & -6.257918 & 1.543637 & 0.942498 \\
\hline 1 & -7.241863 & -0.573375 & -0.385458 & 1 & -7.165013 & -0.411681 & -0.447256 \\
\hline 1 & -6.191930 & -1.614100 & 0.685103 & 1 & -6.157501 & -1.559272 & 0.552147 \\
\hline 6 & 6.460326 & 0.354191 & 0.123841 & 6 & 6.550217 & 0.217038 & 0.126606 \\
\hline 7 & 7.602812 & 0.577846 & 0.156724 & 7 & 7.697357 & 0.415381 & 0.161503 \\
\hline
\end{tabular}

Compound 5b

\begin{tabular}{|c|c|c|c|c|c|c|c|}
\hline & & rotamer A & & & & rotamer B & \\
\hline Atomic number & $\mathbf{x}$ & $\mathbf{y}$ & $\mathbf{z}$ & Atomic number & $\mathbf{x}$ & $\mathbf{y}$ & $\mathbf{z}$ \\
\hline 6 & -2.246234 & -0.834705 & -0.124394 & 6 & -2.189571 & -0.796932 & -0.124819 \\
\hline 6 & -0.990595 & -0.115472 & -0.093289 & 6 & -0.925299 & -0.094136 & -0.109759 \\
\hline 6 & -3.439345 & -0.015466 & -0.163126 & 6 & -3.371320 & 0.034896 & -0.160396 \\
\hline 7 & -0.950893 & 1.219098 & -0.106399 & 7 & -0.859216 & 1.232580 & -0.130973 \\
\hline 7 & -3.377260 & 1.317341 & -0.184066 & 7 & -3.283860 & 1.372941 & -0.190338 \\
\hline 6 & -2.141791 & 1.853836 & -0.153823 & 6 & -2.045978 & 1.888775 & -0.173565 \\
\hline 7 & -4.645175 & -0.613455 & -0.182461 & 7 & -4.580745 & -0.544155 & -0.172865 \\
\hline 1 & -4.624573 & -1.625734 & -0.231967 & 1 & -4.552908 & -1.566980 & -0.189551 \\
\hline 7 & -2.445411 & -2.176708 & -0.131294 & 7 & -2.130417 & -2.152935 & -0.109782 \\
\hline 8 & -1.444170 & -2.949752 & -0.094803 & 8 & -3.205088 & -2.814024 & -0.121338 \\
\hline 7 & 0.140318 & -0.860115 & -0.052549 & 7 & 0.199166 & -0.859588 & -0.072908 \\
\hline 6 & 1.496381 & -0.502194 & -0.021093 & 6 & 1.562057 & -0.534249 & -0.046021 \\
\hline 1 & -0.086935 & -1.867086 & -0.051410 & 1 & -0.025152 & -1.852066 & -0.066612 \\
\hline 6 & 2.414515 & -1.572432 & 0.012258 & 6 & 2.462620 & -1.618200 & -0.032956 \\
\hline 6 & 3.780021 & -1.332996 & 0.045550 & 6 & 3.833379 & -1.402325 & -0.003272 \\
\hline 6 & 4.262615 & -0.016805 & 0.046327 & 6 & 4.337636 & -0.095820 & 0.012326 \\
\hline
\end{tabular}




\begin{tabular}{|c|c|c|c|c|c|c|c|}
\hline 6 & 1.982745 & 0.815204 & -0.020128 & 6 & 2.070486 & 0.775577 & -0.030311 \\
\hline 6 & 3.358851 & 1.045897 & 0.013277 & 6 & 3.449071 & 0.981880 & 0.000665 \\
\hline 7 & -2.088757 & 3.204442 & -0.173912 & 7 & -1.961702 & 3.237797 & -0.202452 \\
\hline 1 & 2.045839 & -2.594203 & 0.011848 & 1 & 2.080163 & -2.635254 & -0.048995 \\
\hline 1 & 4.472212 & -2.168476 & 0.071150 & 1 & 4.511254 & -2.249227 & 0.001774 \\
\hline 1 & 1.286678 & 1.639546 & -0.045353 & 1 & 1.387742 & 1.611335 & -0.044195 \\
\hline 1 & 3.724769 & 2.066604 & 0.013601 & 1 & 3.831435 & 1.996868 & 0.010322 \\
\hline 1 & -2.946747 & 3.731111 & -0.204083 & 1 & -2.807123 & 3.784589 & -0.229468 \\
\hline 1 & -1.197124 & 3.671476 & -0.152498 & 1 & -1.059177 & 3.683643 & -0.191416 \\
\hline 6 & -5.893598 & 0.086409 & -0.306449 & 6 & -5.817296 & 0.182916 & -0.256817 \\
\hline 6 & -6.580895 & 0.617276 & 0.927868 & 6 & -6.517058 & 0.624045 & 1.007468 \\
\hline 6 & -7.126251 & -0.600251 & 0.215649 & 6 & -7.068295 & -0.522048 & 0.188105 \\
\hline 1 & -5.984629 & 0.677273 & -1.214743 & 1 & -5.877995 & 0.849425 & -1.112736 \\
\hline 1 & -6.078093 & 0.462321 & 1.876968 & 1 & -6.034228 & 0.382870 & 1.949121 \\
\hline 1 & -7.115142 & 1.558348 & 0.840916 & 1 & -7.033848 & 1.578716 & 0.989413 \\
\hline 1 & -8.040279 & -0.507547 & -0.362163 & 1 & -7.971172 & -0.366835 & -0.393866 \\
\hline 1 & -6.998926 & -1.564016 & 0.701312 & 1 & -6.963985 & -1.524467 & 0.594183 \\
\hline 6 & 5.745779 & 0.214737 & 0.082216 & 6 & 5.819189 & 0.130187 & 0.097815 \\
\hline 9 & 6.072085 & 1.529809 & 0.088383 & 9 & 6.184713 & 1.357869 & -0.348677 \\
\hline 9 & 6.375944 & -0.342608 & -0.989067 & 9 & 6.521052 & -0.782275 & -0.624255 \\
\hline 9 & 6.322345 & -0.339873 & 1.184522 & 9 & 6.280389 & 0.033928 & 1.378174 \\
\hline
\end{tabular}

Compound 5c

\begin{tabular}{|c|c|c|c|c|c|c|c|}
\hline & & rotamer A & & & & rotamer B & \\
\hline Atomic number & $\mathbf{x}$ & $\mathbf{y}$ & $\mathbf{Z}$ & Atomic number & $\mathbf{x}$ & $\mathbf{y}$ & $\mathbf{Z}$ \\
\hline 6 & -2.258982 & -0.834654 & -0.123884 & 6 & -2.199841 & -0.794593 & -0.121108 \\
\hline 6 & -1.016732 & -0.091826 & -0.091400 & 6 & -0.951070 & -0.063533 & -0.096438 \\
\hline 6 & -3.467339 & -0.037784 & -0.158987 & 6 & -3.399673 & 0.011283 & -0.158077 \\
\hline 7 & -1.003660 & 1.243488 & -0.099831 & 7 & -0.915819 & 1.264600 & -0.109261 \\
\hline 7 & -3.431285 & 1.295763 & -0.175703 & 7 & -3.342649 & 1.350867 & -0.179041 \\
\hline 6 & -2.205530 & 1.855405 & -0.144136 & 6 & -2.116039 & 1.893990 & -0.152224 \\
\hline 7 & -4.662035 & -0.658883 & -0.179048 & 7 & -4.595992 & -0.594932 & -0.180815 \\
\hline 1 & -4.621847 & -1.670296 & -0.233442 & 1 & -4.544711 & -1.616823 & -0.203871 \\
\hline 7 & -2.433911 & -2.179949 & -0.135855 & 7 & -2.111492 & -2.148739 & -0.113504 \\
\hline 8 & -1.418841 & -2.935075 & -0.102917 & 8 & -3.171846 & -2.833329 & -0.134608 \\
\hline 7 & 0.128769 & -0.813648 & -0.054397 & 7 & 0.190160 & -0.802792 & -0.059307 \\
\hline 6 & 1.478119 & -0.431063 & -0.024500 & 6 & 1.545917 & -0.449030 & -0.029057 \\
\hline 1 & -0.077664 & -1.824606 & -0.056277 & 1 & -0.011249 & -1.800138 & -0.057988 \\
\hline 6 & 2.415214 & -1.484333 & 0.004755 & 6 & 2.467587 & -1.515594 & -0.006643 \\
\hline 6 & 3.777435 & -1.221350 & 0.035274 & 6 & 3.833677 & -1.273229 & 0.025951 \\
\hline 6 & 4.242549 & 0.104057 & 0.037177 & 6 & 4.318949 & 0.044610 & 0.037071 \\
\hline 6 & 1.940175 & 0.897771 & -0.022127 & 6 & 2.027755 & 0.872633 & -0.018234 \\
\hline 6 & 3.308981 & 1.148779 & 0.008209 & 6 & 3.399917 & 1.102571 & 0.014487 \\
\hline 7 & -2.178070 & 3.207008 & -0.159242 & 7 & -2.061791 & 3.244831 & -0.170551 \\
\hline 1 & 2.062835 & -2.512157 & 0.003112 & 1 & 2.102543 & -2.539478 & -0.015069 \\
\hline 1 & 4.485979 & -2.041062 & 0.057539 & 1 & 4.529566 & -2.103859 & 0.042871 \\
\hline 1 & 1.228655 & 1.708852 & -0.044191 & 1 & 1.328247 & 1.694321 & -0.035469 \\
\hline 1 & 3.673508 & 2.170700 & 0.009860 & 1 & 3.778950 & 2.119179 & 0.023338 \\
\hline 1 & -3.045647 & 3.717778 & -0.186046 & 1 & -2.918898 & 3.773185 & -0.193837 \\
\hline 1 & -1.294979 & 3.690052 & -0.135594 & 1 & -1.169152 & 3.709905 & -0.147251 \\
\hline 6 & -5.922823 & 0.017929 & -0.303148 & 6 & -5.847541 & 0.105147 & -0.268797 \\
\hline 6 & -6.617497 & 0.539735 & 0.930741 & 6 & -6.560017 & 0.532303 & 0.993000 \\
\hline 6 & -7.142533 & -0.689052 & 0.222475 & 6 & -7.084490 & -0.626087 & 0.173219 \\
\hline 1 & -6.026134 & 0.605082 & -1.212642 & 1 & -5.920627 & 0.769724 & -1.125362 \\
\hline 1 & -6.110246 & 0.396189 & 1.879247 & 1 & -6.074538 & 0.302452 & 1.936116 \\
\hline 1 & -7.168350 & 1.471053 & 0.842303 & 1 & -7.097000 & 1.475729 & 0.972677 \\
\hline 1 & -8.058933 & -0.613650 & -0.354143 & 1 & -7.988918 & -0.490612 & -0.411311 \\
\hline 1 & -6.997615 & -1.649280 & 0.710194 & 1 & -6.959719 & -1.625713 & 0.580369 \\
\hline 8 & 6.483369 & -0.650051 & 0.097635 & 8 & 6.548045 & -0.743158 & 0.093290 \\
\hline 6 & 7.898878 & -0.394662 & 0.129975 & 6 & 7.967043 & -0.508562 & 0.129480 \\
\hline 1 & 8.369789 & -1.377310 & 0.149052 & 1 & 8.423783 & -1.498035 & 0.138995 \\
\hline 1 & 8.206405 & 0.163976 & -0.757380 & 1 & 8.283631 & 0.054625 & -0.751778 \\
\hline 1 & 8.163152 & 0.177753 & 1.022491 & 1 & 8.238665 & 0.050798 & 1.028056 \\
\hline 6 & 5.687823 & 0.446762 & 0.068989 & 6 & 5.768394 & 0.365648 & 0.072688 \\
\hline 8 & 6.131224 & 1.582149 & 0.070030 & 8 & 6.228689 & 1.494306 & 0.083187 \\
\hline
\end{tabular}

Compound 5d

\begin{tabular}{|c|c|c|c|c|c|c|c|}
\hline & & rotamer A & & & & rotamer B & \\
\hline Atomic number & $\mathbf{x}$ & $\mathbf{y}$ & $\mathbf{z}$ & Atomic number & $\mathbf{x}$ & $\mathbf{y}$ & $\mathbf{z}$ \\
\hline 6 & -1.594032 & -0.831639 & -0.121174 & 6 & -1.533628 & -0.793796 & -0.116260 \\
\hline 6 & -0.345040 & -0.099133 & -0.083057 & 6 & -0.273068 & -0.081435 & -0.085877 \\
\hline 6 & -2.795327 & -0.024894 & -0.164686 & 6 & -2.720762 & 0.029759 & -0.162895 \\
\hline
\end{tabular}




\begin{tabular}{|c|c|c|c|c|c|c|c|}
\hline 7 & -0.321437 & 1.237935 & -0.094489 & 7 & -0.219028 & 1.247763 & -0.102548 \\
\hline 7 & -2.748296 & 1.308116 & -0.184165 & 7 & -2.644052 & 1.368315 & -0.187612 \\
\hline 6 & -1.517741 & 1.858003 & -0.147158 & 6 & -1.409420 & 1.893257 & -0.155127 \\
\hline 7 & -3.994912 & -0.636606 & -0.189900 & 7 & -3.925641 & -0.559219 & -0.191171 \\
\hline 1 & -3.962076 & -1.648429 & -0.241891 & 1 & -3.888360 & -1.582007 & -0.210824 \\
\hline 7 & -1.778474 & -2.174708 & -0.131300 & 7 & -1.465787 & -2.148375 & -0.104500 \\
\hline 8 & -0.767650 & -2.936840 & -0.091159 & 8 & -2.536242 & -2.818052 & -0.130147 \\
\hline 7 & 0.791929 & -0.828461 & -0.037848 & 7 & 0.855048 & -0.834472 & -0.039337 \\
\hline 6 & 2.146680 & -0.453238 & -0.000669 & 6 & 2.218713 & -0.495470 & -0.002597 \\
\hline 1 & 0.577204 & -1.838050 & -0.039263 & 1 & 0.641179 & -1.829023 & -0.036656 \\
\hline 6 & 3.078632 & -1.507793 & 0.033734 & 6 & 3.129547 & -1.567793 & 0.022483 \\
\hline 6 & 4.445852 & -1.254662 & 0.071849 & 6 & 4.502000 & -1.342351 & 0.061703 \\
\hline 6 & 4.896334 & 0.066551 & 0.076074 & 6 & 4.979353 & -0.031156 & 0.076368 \\
\hline 6 & 2.616639 & 0.870417 & 0.004416 & 6 & 2.715108 & 0.818164 & 0.012653 \\
\hline 6 & 3.989140 & 1.123358 & 0.042644 & 6 & 4.092410 & 1.042938 & 0.052014 \\
\hline 7 & -1.481969 & 3.210029 & -0.165789 & 7 & -1.337402 & 3.243829 & -0.177853 \\
\hline 1 & 2.726545 & -2.535666 & 0.030215 & 1 & 2.759933 & -2.589913 & 0.010924 \\
\hline 1 & 5.154093 & -2.075277 & 0.097863 & 1 & 5.193091 & -2.177662 & 0.080446 \\
\hline 1 & 1.910608 & 1.686641 & -0.021668 & 1 & 2.025438 & 1.648523 & -0.006558 \\
\hline 1 & 4.348754 & 2.146615 & 0.046224 & 1 & 4.471514 & 2.059074 & 0.063830 \\
\hline 1 & -2.346489 & 3.725491 & -0.198823 & 1 & -2.187647 & 3.782690 & -0.208749 \\
\hline 1 & -0.596463 & 3.688081 & -0.138527 & 1 & -0.439255 & 3.697621 & -0.151439 \\
\hline 6 & -5.249628 & 0.049621 & -0.322115 & 6 & -5.166407 & 0.158292 & -0.288797 \\
\hline 6 & -5.947232 & 0.578833 & 0.907000 & 6 & -5.881523 & 0.597586 & 0.967377 \\
\hline 6 & -6.477606 & -0.647179 & 0.197858 & 6 & -6.416551 & -0.554733 & 0.145803 \\
\hline 1 & -5.343582 & 0.636066 & -1.233098 & 1 & -5.224416 & 0.822475 & -1.146834 \\
\hline 1 & -5.446335 & 0.433068 & 1.858539 & 1 & -5.405750 & 0.362482 & 1.914135 \\
\hline 1 & -6.490606 & 1.514106 & 0.813965 & 1 & -6.405201 & 1.548354 & 0.941910 \\
\hline 1 & -7.390247 & -0.565879 & -0.383909 & 1 & -7.315025 & -0.407581 & -0.445068 \\
\hline 1 & -6.342560 & -1.607622 & 0.687970 & 1 & -6.308607 & -1.555331 & 0.555373 \\
\hline 17 & 6.623203 & 0.397175 & 0.123989 & 17 & 6.712732 & 0.264721 & 0.125926 \\
\hline
\end{tabular}

Compound 5e

\begin{tabular}{|c|c|c|c|c|c|c|c|}
\hline \multicolumn{8}{|l|}{ ompound 5e } \\
\hline & & rotamer $\mathrm{A}$ & & & & rotamer B & \\
\hline Atomic number & $\mathbf{x}$ & $\mathbf{y}$ & $\mathbf{z}$ & Atomic number & $\mathbf{x}$ & $\mathbf{y}$ & $\mathbf{z}$ \\
\hline 6 & -0.813107 & -0.815948 & -0.118049 & 6 & 0.753004 & -0.780265 & -0.109803 \\
\hline 6 & 0.407723 & -0.036678 & -0.063805 & 6 & -0.489753 & -0.035456 & -0.064283 \\
\hline 6 & -2.043776 & -0.055557 & -0.169656 & 6 & 1.960507 & 0.012630 & -0.165951 \\
\hline 7 & 0.378164 & 1.301182 & -0.068308 & 7 & -0.507103 & 1.295594 & -0.075475 \\
\hline 7 & -2.048568 & 1.278090 & -0.182851 & 7 & 1.919403 & 1.352581 & -0.184938 \\
\hline 6 & -0.839504 & 1.874105 & -0.130052 & 6 & 0.698321 & 1.908817 & -0.137314 \\
\hline 7 & -3.219240 & -0.713362 & -0.208445 & 7 & 3.149417 & -0.608127 & -0.208999 \\
\hline 1 & -3.146092 & -1.722746 & -0.266501 & 1 & 3.083886 & -1.629651 & -0.232374 \\
\hline 7 & -0.947807 & -2.164731 & -0.137615 & 7 & 0.651840 & -2.132281 & -0.102715 \\
\hline 8 & 0.089568 & -2.889862 & -0.091315 & 8 & 1.705469 & -2.828931 & -0.142048 \\
\hline 7 & 1.570903 & -0.720370 & -0.011022 & 7 & -1.635582 & -0.757497 & -0.009530 \\
\hline 6 & 2.913418 & -0.296623 & 0.039563 & 6 & -2.993192 & -0.387217 & 0.039061 \\
\hline 1 & 1.397296 & -1.736939 & -0.019006 & 1 & -1.448398 & -1.757282 & -0.011732 \\
\hline 6 & 3.877870 & -1.321815 & 0.082192 & 6 & -3.923306 & -1.443186 & 0.074351 \\
\hline 6 & 5.234612 & -1.015250 & 0.132192 & 6 & -5.289910 & -1.182156 & 0.124203 \\
\hline 6 & 5.657005 & 0.318196 & 0.140993 & 6 & -5.756906 & 0.135882 & 0.140185 \\
\hline 6 & 3.334854 & 1.043442 & 0.048792 & 6 & -3.458943 & 0.937721 & 0.055121 \\
\hline 6 & 4.700532 & 1.334308 & 0.099209 & 6 & -4.833577 & 1.182504 & 0.105432 \\
\hline 7 & -0.856346 & 3.227212 & -0.141520 & 7 & 0.662051 & 3.261448 & -0.153856 \\
\hline 1 & 3.554452 & -2.359410 & 0.075225 & 1 & -3.568441 & -2.470938 & 0.062082 \\
\hline 1 & 5.962228 & -1.820869 & 0.164129 & 1 & -5.989787 & -2.012223 & 0.150423 \\
\hline 1 & 6.714917 & 0.559103 & 0.179812 & 1 & -6.822151 & 0.341345 & 0.179027 \\
\hline 1 & 2.598927 & 1.832467 & 0.016376 & 1 & -2.749609 & 1.751060 & 0.028075 \\
\hline 1 & 5.014823 & 2.374326 & 0.105544 & 1 & -5.181296 & 2.211774 & 0.117392 \\
\hline 1 & -1.739900 & 3.708942 & -0.176778 & 1 & 1.525901 & 3.777892 & -0.187034 \\
\hline 1 & 0.009843 & 3.738253 & -0.097841 & 1 & -0.223722 & 3.737749 & -0.111180 \\
\hline 6 & -4.497410 & -0.075472 & -0.353460 & 6 & 4.407019 & 0.076946 & -0.318975 \\
\hline 6 & -5.221126 & 0.436503 & 0.867545 & 6 & 5.642376 & -0.667243 & 0.105740 \\
\hline 6 & -5.702298 & -0.813205 & 0.164602 & 6 & 5.144396 & 0.499988 & 0.929763 \\
\hline 1 & -4.608353 & 0.500779 & -1.269210 & 1 & 4.474995 & 0.738245 & -1.178635 \\
\hline 1 & -4.720324 & 0.316173 & 1.822650 & 1 & 5.512210 & -1.664010 & 0.518137 \\
\hline 1 & -5.797887 & 1.350525 & 0.764713 & 1 & 6.539121 & -0.544199 & -0.493268 \\
\hline 1 & -6.613893 & $\begin{array}{l}-0.769211 \\
\end{array}$ & -0.422860 & 1 & 5.692138 & 1.436929 & $\begin{array}{l}0.897793 \\
\end{array}$ \\
\hline 1 & -5.535087 & -1.764682 & 0.662205 & 1 & 4.671013 & 0.278940 & 1.881092 \\
\hline
\end{tabular}

Compound $\mathbf{5 f}$

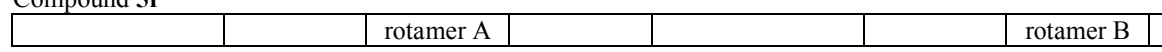




\begin{tabular}{|c|c|c|c|c|c|c|c|}
\hline Atomic number & $\mathbf{x}$ & $\mathbf{y}$ & $\mathbf{z}$ & Atomic number & $\mathbf{x}$ & $\mathbf{y}$ & $\mathbf{z}$ \\
\hline 6 & -1.227317 & -0.826462 & -0.119472 & 6 & 1.166202 & -0.789312 & -0.114780 \\
\hline 6 & 0.012293 & -0.076197 & -0.075159 & 6 & -0.089006 & -0.064022 & -0.078132 \\
\hline 6 & -2.439695 & -0.036963 & -0.165828 & 6 & 2.361185 & 0.022779 & -0.164762 \\
\hline 7 & 0.014098 & 1.262824 & -0.084253 & 7 & -0.126811 & 1.267340 & -0.092695 \\
\hline 7 & -2.413242 & 1.296181 & -0.183302 & 7 & 2.299261 & 1.361758 & -0.187267 \\
\hline 6 & -1.189521 & 1.863585 & -0.140591 & 6 & 1.068780 & 1.898673 & -0.149001 \\
\hline 7 & -3.630677 & -0.667192 & -0.195497 & 7 & 3.559994 & -0.579599 & -0.198334 \\
\hline 1 & -3.581229 & -1.678129 & -0.251184 & 1 & 3.509990 & -1.602185 & -0.219926 \\
\hline 7 & -1.393882 & -2.171333 & -0.133720 & 7 & 1.086978 & -2.142318 & -0.105354 \\
\hline 8 & -0.372807 & -2.920434 & -0.091462 & 8 & 2.152231 & -2.822469 & -0.136583 \\
\hline 7 & 1.158789 & -0.785628 & -0.026969 & 7 & -1.223201 & -0.802054 & -0.028153 \\
\hline 6 & 2.510818 & -0.390666 & 0.015349 & 6 & -2.586717 & -0.449651 & 0.013779 \\
\hline 1 & 0.962193 & -1.798032 & -0.031046 & 1 & -1.022352 & -1.799105 & -0.027522 \\
\hline 6 & 3.456833 & -1.433003 & 0.051843 & 6 & -3.506183 & -1.514748 & 0.043103 \\
\hline 6 & 4.817763 & -1.154314 & 0.095449 & 6 & -4.874227 & -1.270810 & 0.088286 \\
\hline 6 & 5.292007 & 0.167825 & 0.104138 & 6 & -5.382030 & 0.038195 & 0.105517 \\
\hline 6 & 2.967007 & 0.935430 & 0.025100 & 6 & -3.076050 & 0.864062 & 0.031828 \\
\hline 6 & 4.340232 & 1.192836 & 0.069127 & 6 & -4.455444 & 1.086188 & 0.077278 \\
\hline 7 & -1.175396 & 3.217014 & -0.157331 & 7 & 1.012175 & 3.250843 & -0.169893 \\
\hline 1 & 3.115802 & -2.465094 & 0.046650 & 1 & -3.142115 & -2.539438 & 0.031473 \\
\hline 1 & 5.524387 & -1.980302 & 0.123980 & 1 & -5.559154 & -2.115061 & 0.111212 \\
\hline 1 & 2.253649 & 1.745355 & -0.000691 & 1 & -2.383210 & 1.691869 & 0.011434 \\
\hline 1 & 4.673372 & 2.227979 & 0.076922 & 1 & -4.813682 & 2.112842 & 0.091855 \\
\hline 1 & -2.047794 & 3.718719 & -0.190442 & 1 & 1.868300 & 3.780104 & -0.200050 \\
\hline 1 & -0.297258 & 3.707931 & -0.123313 & 1 & 0.118914 & 3.713576 & -0.135562 \\
\hline 6 & -4.894151 & 0.000140 & -0.335172 & 6 & 4.807462 & 0.124632 & -0.300605 \\
\hline 6 & -5.603181 & 0.525005 & 0.889133 & 6 & 6.051296 & -0.600111 & 0.133021 \\
\hline 6 & -6.114048 & -0.711657 & 0.183922 & 6 & 5.529942 & 0.560063 & 0.952612 \\
\hline 1 & -4.994334 & 0.581555 & -1.248883 & 1 & 4.871144 & 0.786258 & -1.160351 \\
\hline 1 & -5.102794 & 0.390747 & 1.842611 & 1 & 5.933694 & -1.598451 & 0.545397 \\
\hline 1 & -6.159584 & 1.452018 & 0.790270 & 1 & 6.950109 & -0.463781 & -0.460007 \\
\hline 1 & -7.026000 & -0.645628 & -0.400933 & 1 & 6.063556 & 1.505228 & 0.923465 \\
\hline 1 & -5.966952 & -1.667999 & 0.678549 & 1 & 5.053522 & 0.332607 & 1.900908 \\
\hline 6 & 6.773514 & 0.461572 & 0.141578 & 6 & -6.870610 & 0.294690 & 0.144104 \\
\hline 1 & 7.261628 & -0.049034 & 0.979754 & 1 & -7.358402 & -0.042373 & -0.778788 \\
\hline 1 & 7.270671 & 0.123874 & -0.776043 & 1 & -7.347583 & -0.238267 & 0.974721 \\
\hline 1 & 6.963553 & 1.533657 & 0.246506 & 1 & -7.086958 & 1.360369 & 0.262782 \\
\hline
\end{tabular}

Compound 5g

\begin{tabular}{|c|c|c|c|c|c|c|c|}
\hline & & rotamer $\mathrm{A}$ & & & & rotamer B & \\
\hline Atomic number & $\mathbf{x}$ & $\mathbf{y}$ & $\mathbf{z}$ & Atomic number & $\mathbf{x}$ & $\mathbf{y}$ & $\mathbf{z}$ \\
\hline 6 & -1.210334 & -0.827903 & -0.118990 & 6 & 1.150199 & -0.791287 & -0.112480 \\
\hline 6 & 0.029159 & -0.077423 & -0.073698 & 6 & -0.105960 & -0.067163 & -0.075662 \\
\hline 6 & -2.422744 & -0.038651 & -0.166045 & 6 & 2.344157 & 0.021924 & -0.164383 \\
\hline 7 & 0.030923 & 1.262953 & -0.082055 & 7 & -0.145004 & 1.265339 & -0.091592 \\
\hline 7 & -2.396405 & 1.294454 & -0.182796 & 7 & 2.280936 & 1.360881 & -0.188375 \\
\hline 6 & -1.172802 & 1.862545 & -0.138737 & 6 & 1.050026 & 1.896927 & -0.149766 \\
\hline 7 & -3.613387 & -0.669325 & $\begin{array}{l}-0.197043 \\
\end{array}$ & 7 & 3.543343 & -0.579540 & -0.198423 \\
\hline 1 & -3.563342 & -1.680246 & -0.253008 & 1 & 3.493802 & -1.602282 & -0.218788 \\
\hline 7 & -1.375029 & -2.172396 & -0.133623 & 7 & 1.072146 & -2.144018 & -0.101891 \\
\hline 8 & -0.351643 & -2.919608 & -0.090822 & 8 & 2.137973 & -2.823701 & -0.133279 \\
\hline 7 & 1.174587 & -0.785932 & -0.025440 & 7 & -1.238620 & -0.804641 & -0.024230 \\
\hline 6 & 2.526810 & -0.389423 & 0.016916 & 6 & -2.603228 & -0.450752 & 0.016582 \\
\hline 1 & 0.977067 & -1.798844 & -0.029798 & 1 & -1.037870 & -1.801709 & -0.023117 \\
\hline 6 & 3.476284 & -1.428925 & 0.055299 & 6 & -3.526247 & -1.513003 & 0.039167 \\
\hline 6 & 4.837889 & -1.158153 & 0.097556 & 6 & -4.894972 & -1.276905 & 0.082059 \\
\hline 6 & 5.282785 & 0.168552 & 0.102614 & 6 & -5.373422 & 0.037305 & 0.103435 \\
\hline 6 & 2.979881 & 0.938104 & 0.022772 & 6 & -3.089173 & 0.864297 & 0.038728 \\
\hline 6 & 4.350029 & 1.207323 & 0.065309 & 6 & -4.466067 & 1.098385 & 0.081443 \\
\hline 7 & -1.161175 & 3.216433 & -0.154918 & 7 & 0.993921 & 3.249471 & -0.172316 \\
\hline 1 & 3.138177 & -2.461682 & 0.051337 & 1 & -3.165476 & -2.538449 & 0.022477 \\
\hline 1 & 5.564884 & -1.962772 & 0.126593 & 1 & -5.600967 & -2.100368 & 0.098946 \\
\hline 1 & 2.263926 & 1.745710 & -0.006195 & 1 & -2.393610 & 1.689950 & 0.021636 \\
\hline 1 & 4.687347 & 2.241812 & 0.069002 & 1 & -4.827934 & 2.124376 & 0.097905 \\
\hline 1 & -2.034852 & 3.716062 & -0.185261 & 1 & 1.850461 & 3.777896 & -0.204924 \\
\hline 1 & -0.284613 & 3.709593 & -0.116584 & 1 & 0.101275 & 3.713208 & -0.139155 \\
\hline 6 & -4.877088 & -0.002596 & -0.337388 & 6 & 4.790204 & 0.125389 & -0.302994 \\
\hline 6 & -5.587483 & 0.521156 & 0.886617 & 6 & 6.035259 & -0.598511 & 0.128468 \\
\hline 6 & -6.097094 & -0.715308 & 0.180155 & 6 & 5.514735 & 0.561489 & 0.948838 \\
\hline 1 & -4.976768 & 0.579321 & -1.250835 & 1 & 4.851928 & 0.786913 & -1.162958 \\
\hline 1 & -5.087905 & 0.386508 & 1.840464 & 1 & 5.919000 & -1.596844 & 0.541233 \\
\hline
\end{tabular}




\begin{tabular}{|c|c|c|c|c|c|c|c|}
\hline 1 & -6.144286 & 1.447942 & 0.787854 & 1 & 6.932896 & -0.461764 & -0.466238 \\
\hline 1 & -7.008542 & -0.649351 & -0.405489 & 1 & 6.047753 & 1.506959 & 0.918553 \\
\hline 1 & -5.949978 & -1.671892 & 0.674300 & 1 & 5.040249 & 0.333863 & 1.898062 \\
\hline 8 & 6.637530 & 0.380844 & 0.144675 & 8 & -6.733967 & 0.214886 & 0.145305 \\
\hline 1 & 6.823439 & 1.328844 & 0.143650 & 1 & -6.943695 & 1.157761 & 0.157326 \\
\hline
\end{tabular}

Compound 5h

\begin{tabular}{|c|c|c|c|c|c|c|c|}
\hline & & rotamer $\mathrm{A}$ & & & & rotamer B & \\
\hline Atomic number & $\mathbf{x}$ & $\mathbf{y}$ & $\mathbf{Z}$ & Atomic number & $\mathbf{x}$ & $\mathbf{y}$ & $\mathbf{z}$ \\
\hline 6 & -1.218557 & -0.828082 & -0.121270 & 6 & -1.158534 & -0.791332 & -0.116314 \\
\hline 6 & 0.021678 & -0.077826 & -0.071918 & 6 & 0.098819 & -0.067244 & -0.074502 \\
\hline 6 & -2.430572 & -0.037884 & -0.166308 & 6 & -2.352121 & 0.023078 & -0.164628 \\
\hline 7 & 0.022442 & 1.263881 & -0.074801 & 7 & 0.136545 & 1.266486 & -0.083066 \\
\hline 7 & -2.404689 & 1.294821 & -0.176941 & 7 & -2.289381 & 1.361835 & -0.179696 \\
\hline 6 & -1.179990 & 1.862749 & -0.128663 & 6 & -1.057460 & 1.897406 & -0.136351 \\
\hline 7 & -3.621504 & -0.669132 & -0.200750 & 7 & -3.551337 & -0.578868 & -0.203458 \\
\hline 1 & -3.570366 & -1.679686 & -0.261905 & 1 & -3.500454 & -1.601679 & -0.229787 \\
\hline 7 & -1.383740 & -2.171672 & -0.141565 & 7 & -1.082145 & -2.143340 & -0.113210 \\
\hline 8 & -0.359424 & -2.919569 & -0.099843 & 8 & -2.149172 & -2.822937 & -0.149711 \\
\hline 7 & 1.166423 & -0.784826 & -0.025272 & 7 & 1.230588 & -0.802921 & $\begin{array}{l}-0.025940 \\
\end{array}$ \\
\hline 6 & 2.519269 & $\begin{array}{l}-0.388969 \\
\end{array}$ & 0.019408 & 6 & 2.596492 & -0.449747 & 0.017737 \\
\hline 1 & 0.968884 & -1.797761 & -0.031081 & 1 & 1.030423 & -1.800084 & -0.025099 \\
\hline 6 & 3.468502 & -1.424954 & 0.082895 & 6 & 3.518637 & -1.508542 & 0.077911 \\
\hline 6 & 4.829917 & -1.151684 & 0.124429 & 6 & 4.887525 & -1.269927 & 0.120257 \\
\hline 6 & 5.296629 & 0.174532 & 0.102417 & 6 & 5.387993 & 0.043111 & 0.103121 \\
\hline 6 & 2.978720 & 0.937643 & -0.000153 & 6 & 3.088968 & 0.864193 & 0.002228 \\
\hline 6 & 4.345947 & 1.205773 & 0.041007 & 6 & 4.463052 & 1.097104 & 0.044808 \\
\hline 7 & -1.169727 & 3.217509 & -0.141528 & 7 & -1.002376 & 3.250773 & -0.153006 \\
\hline 1 & 3.132222 & -2.458420 & 0.098511 & 1 & 3.159350 & -2.534823 & 0.090632 \\
\hline 1 & 5.538889 & -1.973857 & 0.179085 & 1 & 5.574580 & -2.110663 & 0.172493 \\
\hline 1 & 2.264940 & 1.746441 & -0.049072 & 1 & 2.395934 & 1.691056 & -0.044198 \\
\hline 1 & 4.679961 & 2.240615 & 0.029537 & 1 & 4.822056 & 2.123563 & 0.037138 \\
\hline 1 & -2.044811 & 3.715518 & -0.137665 & 1 & -1.860317 & 3.777887 & -0.147381 \\
\hline 1 & -0.295165 & 3.709934 & -0.065194 & 1 & -0.111763 & 3.713625 & -0.077367 \\
\hline 6 & -4.884686 & -0.002204 & -0.341326 & 6 & -4.797906 & 0.126405 & -0.305216 \\
\hline 6 & -5.596539 & 0.519149 & 0.882828 & 6 & -5.523545 & 0.556028 & 0.948208 \\
\hline 6 & -6.105501 & -0.715874 & 0.173213 & 6 & -6.043380 & -0.599776 & 0.121320 \\
\hline 1 & -4.983703 & 0.582007 & -1.253471 & 1 & -4.859305 & 0.792780 & -1.161517 \\
\hline 1 & -5.097852 & 0.382547 & 1.836857 & 1 & -5.049725 & 0.323494 & 1.896577 \\
\hline 1 & -6.153246 & 1.446154 & 0.785343 & 1 & -6.056697 & 1.501586 & 0.922452 \\
\hline 1 & -7.016374 & -0.648528 & -0.413222 & 1 & -6.940663 & -0.459948 & -0.473259 \\
\hline 1 & -5.958955 & -1.673524 & 0.665456 & 1 & -5.927280 & -1.600251 & 0.528909 \\
\hline 7 & 6.662892 & 0.456991 & 0.200661 & 7 & 6.762438 & 0.291294 & 0.204627 \\
\hline 1 & 6.950586 & 1.360360 & -0.150013 & 1 & 7.070364 & 1.187205 & -0.148511 \\
\hline 1 & 7.286799 & -0.282448 & -0.092404 & 1 & 7.365319 & -0.461599 & -0.098617 \\
\hline
\end{tabular}

Compound 6a

\begin{tabular}{|c|c|c|c|c|c|c|c|}
\hline & & rotamer A & & & & rotamer B & \\
\hline Atomic number & $\mathbf{x}$ & $\mathbf{y}$ & $\mathbf{z}$ & Atomic number & $\mathbf{x}$ & $\mathbf{y}$ & $\mathbf{z}$ \\
\hline 6 & -2.386071 & 0.923864 & 0.000000 & 6 & 2.352261 & -0.758291 & -0.000003 \\
\hline 6 & -1.171276 & 0.137067 & 0.000000 & 6 & 1.090656 & -0.052957 & -0.000002 \\
\hline 6 & -3.616986 & 0.173013 & 0.000000 & 6 & 3.532175 & 0.068302 & -0.000001 \\
\hline 7 & -1.204499 & -1.196714 & 0.000000 & 7 & 1.027895 & 1.273598 & -0.000001 \\
\hline 7 & -3.631748 & -1.161103 & 0.000000 & 7 & 3.451964 & 1.405869 & 0.000001 \\
\hline 6 & -2.429198 & -1.767664 & 0.000000 & 6 & 2.216378 & 1.928694 & 0.000000 \\
\hline 7 & -4.795723 & 0.817003 & 0.000000 & 7 & 4.745077 & -0.496810 & 0.000000 \\
\hline 1 & -4.808657 & 1.826648 & 0.000000 & 1 & 4.792636 & -1.513380 & 0.000006 \\
\hline 7 & -2.517543 & 2.277290 & 0.000000 & 7 & 2.295824 & -2.118085 & 0.000000 \\
\hline 8 & -1.476919 & 2.993014 & 0.000000 & 8 & 3.369368 & -2.773001 & 0.000002 \\
\hline 7 & 0.000000 & 0.820178 & 0.000000 & 7 & -0.036434 & -0.817576 & -0.000003 \\
\hline 6 & 1.333371 & 0.390248 & 0.000000 & 6 & -1.397298 & -0.490141 & -0.000002 \\
\hline 1 & -0.171454 & 1.837643 & 0.000000 & 1 & 0.185629 & -1.810623 & -0.000003 \\
\hline 6 & 2.309478 & 1.408218 & 0.000000 & 6 & -2.300764 & -1.572638 & 0.000000 \\
\hline 6 & 3.660611 & 1.097422 & 0.000000 & 6 & -3.670294 & -1.356221 & 0.000001 \\
\hline 6 & 4.074380 & -0.247763 & 0.000000 & 6 & -4.177204 & -0.043588 & 0.000001 \\
\hline 6 & 1.745372 & -0.954790 & 0.000000 & 6 & -1.901952 & 0.822927 & -0.000003 \\
\hline 6 & 3.102685 & -1.262030 & 0.000000 & 6 & -3.277078 & 1.034950 & -0.000002 \\
\hline 7 & -2.451102 & -3.118300 & 0.000000 & 7 & 2.137972 & 3.277250 & 0.000001 \\
\hline 1 & 1.997012 & 2.448589 & 0.000000 & 1 & -1.920026 & -2.590411 & 0.000001 \\
\hline 1 & 4.400886 & 1.890236 & 0.000000 & 1 & -4.353245 & -2.198949 & 0.000003 \\
\hline 1 & 1.003124 & -1.738201 & 0.000000 & 1 & -1.215552 & 1.655826 & -0.000004 \\
\hline 1 & 3.415940 & -2.300887 & 0.000000 & 1 & -3.660769 & 2.049888 & -0.000002 \\
\hline
\end{tabular}




\begin{tabular}{|c|c|c|c|c|c|c|c|}
\hline 1 & -3.336729 & -3.598259 & 0.000000 & 1 & 2.985263 & 3.822085 & 0.000003 \\
\hline 1 & -1.586324 & -3.633884 & 0.000000 & 1 & 1.237112 & 3.726725 & 0.000001 \\
\hline 1 & -5.647739 & 0.278518 & 0.000000 & 1 & 5.558626 & 0.101231 & 0.000014 \\
\hline 6 & 5.468377 & -0.578518 & 0.000000 & 6 & -5.590639 & 0.189137 & 0.000002 \\
\hline 7 & 6.601305 & -0.848199 & 0.000000 & 7 & -6.739736 & 0.378690 & 0.000003 \\
\hline
\end{tabular}

Compound 6b

\begin{tabular}{|c|c|c|c|c|c|c|c|}
\hline & & rotamer $\mathrm{A}$ & & & & rotamer B & \\
\hline Atomic number & $\mathbf{x}$ & $\mathbf{y}$ & $\mathbf{z}$ & Atomic number & $\mathbf{x}$ & $\mathbf{y}$ & $\mathbf{z}$ \\
\hline 6 & 3.212490 & -0.815997 & -0.000001 & 6 & -3.149112 & -0.753306 & 0.003710 \\
\hline 6 & 1.952841 & -0.101831 & -0.000002 & 6 & -1.878402 & -0.062624 & -0.006640 \\
\hline 6 & 4.397089 & 0.005739 & 0.000002 & 6 & -4.319243 & 0.086972 & 0.011162 \\
\hline 7 & 1.909205 & 1.232407 & 0.000000 & 7 & -1.801787 & 1.263907 & -0.009792 \\
\hline 7 & 4.334414 & 1.338332 & 0.000003 & 7 & -4.224250 & 1.423514 & 0.007479 \\
\hline 6 & 3.097856 & 1.873241 & 0.000003 & 6 & -2.982244 & 1.931740 & -0.003181 \\
\hline 7 & 5.611688 & -0.568550 & 0.000002 & 7 & -5.538591 & -0.464575 & 0.021936 \\
\hline 1 & 5.682878 & -1.575731 & 0.000002 & 1 & -5.596784 & -1.480724 & 0.024429 \\
\hline 7 & 3.423573 & -2.159015 & -0.000001 & 7 & -3.109241 & -2.113163 & 0.005700 \\
\hline 8 & 2.426756 & -2.934981 & -0.000003 & 8 & -4.190856 & -2.755714 & 0.015214 \\
\hline 7 & 0.824212 & -0.850469 & -0.000004 & 7 & -0.760958 & -0.838232 & -0.013297 \\
\hline 6 & -0.533998 & -0.497062 & -0.000005 & 6 & 0.605714 & -0.524785 & -0.020371 \\
\hline 1 & 1.052959 & -1.856102 & -0.000003 & 1 & -0.992390 & -1.828920 & -0.010898 \\
\hline 6 & -1.448810 & -1.570403 & 0.000004 & 6 & 1.496364 & -1.616557 & -0.032662 \\
\hline 6 & -2.815548 & -1.335381 & 0.000004 & 6 & 2.869391 & -1.412633 & -0.039730 \\
\hline 6 & -3.302132 & -0.020725 & -0.000006 & 6 & 3.384983 & -0.110607 & -0.036770 \\
\hline 6 & -1.024139 & 0.818745 & -0.000015 & 6 & 1.125311 & 0.780567 & -0.017367 \\
\hline 6 & -2.401399 & 1.044994 & -0.000015 & 6 & 2.505914 & 0.974831 & -0.023586 \\
\hline 7 & 3.041106 & 3.223287 & 0.000005 & 7 & -2.888914 & 3.279719 & -0.007700 \\
\hline 1 & -1.077117 & -2.591088 & 0.000012 & 1 & 1.104908 & -2.630288 & -0.039130 \\
\hline 1 & -3.505432 & -2.173138 & 0.000011 & 1 & 3.539735 & -2.265360 & -0.053557 \\
\hline 1 & -0.330457 & 1.645439 & -0.000022 & 1 & 0.449792 & 1.622225 & -0.011905 \\
\hline 1 & -2.770520 & 2.064530 & -0.000023 & 1 & 2.897332 & 1.986393 & -0.023793 \\
\hline 1 & 3.897133 & 3.754093 & 0.000010 & 1 & -3.730016 & 3.833925 & -0.004732 \\
\hline 1 & 2.147429 & 3.686911 & 0.000008 & 1 & -1.982907 & 3.718554 & -0.017781 \\
\hline 1 & 6.430721 & 0.018760 & 0.000005 & 1 & -6.345207 & 0.142695 & 0.027113 \\
\hline 6 & -4.786663 & 0.206148 & 0.000002 & 6 & 4.870183 & 0.104373 & 0.009323 \\
\hline 9 & -5.116901 & 1.520136 & -0.000056 & 9 & 5.233342 & 1.323475 & -0.461952 \\
\hline 9 & -5.388010 & -0.351715 & 1.087205 & 9 & 5.546901 & -0.822520 & -0.717799 \\
\hline 9 & -5.388038 & -0.351819 & -1.087132 & 9 & 5.361471 & 0.021400 & 1.279189 \\
\hline
\end{tabular}

Compound 6c

\begin{tabular}{|c|c|c|c|c|c|c|c|}
\hline & & rotamer A & & & & rotamer B & \\
\hline Atomic number & $\mathbf{x}$ & $\mathbf{y}$ & $\mathbf{z}$ & Atomic number & $\mathbf{x}$ & $\mathbf{y}$ & $\mathbf{z}$ \\
\hline 6 & 3.235403 & -0.852273 & -0.000003 & 6 & 3.169366 & -0.792160 & -0.000036 \\
\hline 6 & 1.995058 & -0.104313 & 0.000002 & 6 & 1.920497 & -0.061356 & -0.000089 \\
\hline 6 & 4.441732 & -0.062599 & -0.000005 & 6 & 4.365534 & 0.011169 & -0.000028 \\
\hline 7 & 1.988677 & 1.230819 & 0.000010 & 7 & 1.887052 & 1.267099 & -0.000049 \\
\hline 7 & 4.415742 & 1.270996 & 0.000005 & 7 & 4.313437 & 1.349857 & 0.000140 \\
\hline 6 & 3.193411 & 1.838977 & 0.000014 & 6 & 3.087395 & 1.896862 & 0.000109 \\
\hline 7 & 5.640565 & -0.670027 & -0.000033 & 7 & 5.566619 & -0.579556 & -0.000487 \\
\hline 1 & 5.683806 & -1.678742 & -0.000036 & 1 & 5.590499 & -1.597182 & 0.000973 \\
\hline 7 & 3.411315 & -2.200240 & 0.000004 & 7 & 3.087782 & -2.149736 & 0.000067 \\
\hline 8 & 2.394587 & -2.950208 & 0.000005 & 8 & 4.149565 & -2.825813 & 0.000170 \\
\hline 7 & 0.846238 & -0.821119 & -0.000002 & 7 & 0.779147 & -0.800343 & -0.000153 \\
\hline 6 & -0.502362 & -0.432784 & -0.000002 & 6 & -0.577557 & -0.446150 & -0.000121 \\
\hline 1 & 1.046485 & -1.832398 & -0.000003 & 1 & 0.978718 & -1.797893 & -0.000114 \\
\hline 6 & -1.443891 & -1.482231 & 0.000015 & 6 & -1.499179 & -1.512682 & 0.000001 \\
\hline 6 & -2.805407 & -1.213564 & 0.000014 & 6 & -2.865693 & -1.270144 & 0.000035 \\
\hline 6 & -3.264677 & 0.113788 & -0.000006 & 6 & -3.350700 & 0.047732 & -0.000056 \\
\hline 6 & -0.958349 & 0.897937 & -0.000021 & 6 & -1.058876 & 0.875503 & -0.000218 \\
\hline 6 & -2.326486 & 1.154672 & -0.000023 & 6 & -2.431472 & 1.105648 & -0.000186 \\
\hline 7 & 3.173132 & 3.190253 & 0.000029 & 7 & 3.036608 & 3.247232 & 0.000276 \\
\hline 1 & -1.095940 & -2.511586 & 0.000031 & 1 & -1.134230 & -2.536641 & 0.000074 \\
\hline 1 & -3.517722 & -2.030286 & 0.000027 & 1 & -3.561945 & -2.100617 & 0.000135 \\
\hline 1 & -0.243277 & 1.706168 & -0.000034 & 1 & -0.359202 & 1.697218 & -0.000316 \\
\hline 1 & -2.686731 & 2.178099 & -0.000039 & 1 & -2.810548 & 2.122265 & -0.000262 \\
\hline 1 & 4.042962 & 3.697994 & 0.000030 & 1 & 3.894562 & 3.774874 & 0.000271 \\
\hline 1 & 2.291853 & 3.677190 & 0.000035 & 1 & 2.144404 & 3.713785 & 0.000195 \\
\hline 8 & -5.509398 & -0.630864 & 0.000012 & 8 & -5.580506 & -0.739737 & 0.000139 \\
\hline 6 & -6.924429 & -0.370076 & 0.000012 & 6 & -7.000169 & -0.505496 & 0.000191 \\
\hline 1 & -7.399329 & -1.350970 & 0.000029 & 1 & -7.456672 & -1.495099 & 0.000327 \\
\hline 1 & -7.208035 & 0.196527 & 0.890270 & 1 & -7.294348 & 0.055779 & 0.890385 \\
\hline
\end{tabular}




\begin{tabular}{|c|c|c|c|c|c|c|c|}
\hline 1 & -7.208041 & 0.196501 & -0.890260 & 1 & -7.294447 & 0.055578 & -0.890097 \\
\hline 6 & -4.709123 & 0.462601 & -0.000008 & 6 & -4.800865 & 0.368951 & -0.000018 \\
\hline 8 & -5.147211 & 1.599959 & -0.000025 & 8 & -5.260726 & 1.497730 & -0.000112 \\
\hline 1 & 6.475174 & -0.105186 & -0.000008 & 1 & 6.392341 & 0.001368 & 0.000777 \\
\hline
\end{tabular}

Compound 6d

\begin{tabular}{|c|c|c|c|c|c|c|c|}
\hline & & rotamer A & & & & rotamer B & \\
\hline Atomic number & $\mathbf{x}$ & $\mathbf{y}$ & $\mathbf{Z}$ & Atomic number & $\mathbf{x}$ & $\mathbf{y}$ & $\mathbf{Z}$ \\
\hline 6 & -2.333196 & 1.353284 & 0.000000 & 6 & -2.499553 & -0.761586 & -0.000014 \\
\hline 6 & -1.266046 & 0.372910 & 0.000000 & 6 & -1.233374 & -0.059205 & -0.000010 \\
\hline 6 & -3.672634 & 0.819994 & 0.000000 & 6 & -3.677002 & 0.068414 & -0.000002 \\
\hline 7 & -1.526993 & -0.938521 & 0.000000 & 7 & -1.171047 & 1.269927 & -0.000009 \\
\hline 7 & -3.913148 & -0.491860 & 0.000000 & 7 & -3.595168 & 1.405680 & 0.000000 \\
\hline 6 & -2.828297 & -1.292496 & 0.000000 & 6 & -2.356912 & 1.925033 & -0.000009 \\
\hline 7 & -4.726141 & 1.654625 & 0.000000 & 7 & -4.891129 & -0.495431 & 0.000037 \\
\hline 1 & -4.566758 & 2.651633 & 0.000000 & 1 & -4.937484 & -1.512443 & 0.000007 \\
\hline 7 & -2.235427 & 2.708172 & 0.000000 & 7 & -2.448829 & -2.119977 & 0.000001 \\
\hline 8 & -1.087846 & 3.238872 & 0.000000 & 8 & -3.525739 & -2.772748 & 0.000001 \\
\hline 7 & 0.000000 & 0.844975 & 0.000000 & 7 & -0.110535 & -0.820670 & -0.000010 \\
\hline 6 & 1.245900 & 0.191826 & 0.000000 & 6 & 1.256695 & -0.491647 & -0.000006 \\
\hline 1 & 0.005704 & 1.876168 & 0.000000 & 1 & -0.330103 & -1.813816 & -0.000014 \\
\hline 6 & 2.379665 & 1.026030 & 0.000000 & 6 & 2.159554 & -1.570770 & -0.000018 \\
\hline 6 & 3.663193 & 0.490083 & 0.000000 & 6 & 3.534173 & -1.355389 & -0.000013 \\
\hline 6 & 3.824727 & -0.896445 & 0.000000 & 6 & 4.021419 & -0.047734 & 0.000004 \\
\hline 6 & 1.425663 & -1.201023 & 0.000000 & 6 & 1.762624 & 0.818246 & 0.000012 \\
\hline 6 & 2.714393 & -1.737957 & 0.000000 & 6 & 3.142089 & 1.032908 & 0.000017 \\
\hline 7 & -3.080910 & -2.620800 & 0.000000 & 7 & -2.278082 & 3.274697 & -0.000019 \\
\hline 1 & 2.252579 & 2.105083 & 0.000000 & 1 & 1.782325 & -2.590168 & -0.000032 \\
\hline 1 & 4.529017 & 1.142786 & 0.000000 & 1 & 4.219341 & -2.195758 & -0.000023 \\
\hline 1 & 0.563124 & -1.850107 & 0.000000 & 1 & 1.078947 & 1.653740 & 0.000022 \\
\hline 1 & 2.849726 & -2.814108 & 0.000000 & 1 & 3.528812 & 2.046222 & 0.000031 \\
\hline 1 & -4.035342 & -2.942277 & 0.000000 & 1 & -3.125125 & 3.819588 & -0.000009 \\
\hline 1 & -2.316214 & -3.275518 & 0.000000 & 1 & -1.376705 & 3.722889 & -0.000021 \\
\hline 1 & -5.656773 & 1.267971 & 0.000000 & 1 & -5.703710 & 0.103752 & 0.000086 \\
\hline 17 & 5.443284 & -1.583881 & 0.000000 & 17 & 5.757345 & 0.235223 & 0.000010 \\
\hline
\end{tabular}

Compound $\mathbf{6 e}$

\begin{tabular}{|c|c|c|c|c|c|c|c|}
\hline & & rotamer A & & & & rotamer B & \\
\hline Atomic number & $\mathbf{x}$ & $\mathbf{y}$ & $\mathbf{Z}$ & Atomic number & $\mathbf{x}$ & $\mathbf{y}$ & $\mathbf{Z}$ \\
\hline 6 & -1.745729 & -0.905225 & -0.000001 & 6 & 1.695670 & -0.797732 & -0.000040 \\
\hline 6 & -0.552441 & -0.081538 & -0.000001 & 6 & 0.458770 & -0.042445 & -0.000074 \\
\hline 6 & -2.998600 & -0.192514 & 0.000000 & 6 & 2.907069 & -0.017836 & -0.000070 \\
\hline 7 & -0.631990 & 1.254031 & -0.000001 & 7 & 0.454732 & 1.288932 & -0.000053 \\
\hline 7 & -3.056970 & 1.139739 & -0.000002 & 7 & 2.882621 & 1.321536 & 0.000076 \\
\hline 6 & -1.871237 & 1.782969 & -0.000001 & 6 & 1.666478 & 1.892096 & 0.000065 \\
\hline 7 & -4.157395 & -0.874520 & 0.000004 & 7 & 4.096091 & -0.633433 & -0.000514 \\
\hline 1 & -4.136365 & -1.883592 & 0.000003 & 1 & 4.096824 & -1.651687 & 0.000888 \\
\hline 7 & -1.837594 & -2.260375 & 0.000000 & 7 & 1.589484 & -2.152345 & 0.000091 \\
\hline 8 & -0.775303 & -2.946111 & -0.000001 & 8 & 2.639201 & -2.849527 & 0.000175 \\
\hline 7 & 0.635866 & -0.722166 & 0.000000 & 7 & -0.694039 & -0.754543 & -0.000106 \\
\hline 6 & 1.963493 & -0.249931 & 0.000000 & 6 & -2.049813 & -0.372577 & -0.000056 \\
\hline 1 & 0.500850 & -1.743779 & 0.000000 & 1 & -0.516947 & -1.756021 & -0.000068 \\
\hline 6 & 2.964902 & -1.239772 & -0.000002 & 6 & -2.989183 & -1.420710 & 0.000042 \\
\hline 6 & 4.310587 & -0.884421 & -0.000002 & 6 & -4.354440 & -1.147998 & 0.000086 \\
\hline 6 & 4.684254 & 0.463513 & 0.000001 & 6 & -4.810210 & 0.174024 & 0.000033 \\
\hline 6 & 2.335711 & 1.104509 & 0.000003 & 6 & -2.504028 & 0.956255 & -0.000109 \\
\hline 6 & 3.690923 & 1.444510 & 0.000003 & 6 & -3.877451 & 1.212795 & -0.000066 \\
\hline 7 & -1.939539 & 3.133978 & -0.000002 & 7 & 1.645469 & 3.244440 & 0.000220 \\
\hline 1 & 2.679150 & -2.288406 & -0.000004 & 1 & -2.642828 & -2.451452 & 0.000084 \\
\hline 1 & 5.067606 & -1.663084 & -0.000004 & 1 & -5.061935 & -1.971962 & 0.000163 \\
\hline 1 & 5.733446 & 0.742627 & 0.000001 & 1 & -5.874353 & 0.388606 & 0.000066 \\
\hline 1 & 1.570864 & 1.866208 & 0.000005 & 1 & -1.787419 & 1.763630 & -0.000185 \\
\hline 1 & 3.967575 & 2.495159 & 0.000005 & 1 & -4.216613 & 2.244955 & -0.000111 \\
\hline 1 & -2.840843 & 3.583122 & -0.000003 & 1 & 2.514886 & 3.752685 & 0.000118 \\
\hline 1 & -1.091911 & 3.677001 & -0.000004 & 1 & 0.763569 & 3.729803 & 0.000080 \\
\hline 1 & -5.025775 & -0.363772 & 0.000005 & 1 & 4.933534 & -0.069679 & 0.000706 \\
\hline
\end{tabular}

Compound $\mathbf{6 f}$

\begin{tabular}{|c|c|c|c|c|c|c|c|} 
& & rotamer A & & & & rotamer B & \\
\hline Atomic number & $\mathbf{x}$ & $\mathbf{y}$ & $\mathbf{z}$ & Atomic number & $\mathbf{x}$ & $\mathbf{y}$ & $\mathbf{z}$ \\
\hline 6 & -2.190493 & -0.858312 & 0.000087 & 6 & 2.127114 & -0.775315 & 0.000034 \\
\hline
\end{tabular}




\begin{tabular}{|c|c|c|c|c|c|c|c|}
\hline 6 & -0.957676 & -0.094462 & -0.000111 & 6 & 0.869372 & -0.054062 & -0.000069 \\
\hline 6 & -3.406607 & -0.084277 & 0.000445 & 6 & 3.316496 & 0.037780 & 0.000315 \\
\hline 7 & -0.971689 & 1.244224 & 0.000221 & 7 & 0.829393 & 1.277477 & 0.000179 \\
\hline 7 & -3.399340 & 1.249088 & 0.000784 & 7 & 3.255570 & 1.375892 & 0.000593 \\
\hline 6 & -2.182799 & 1.832980 & 0.000742 & 6 & 2.023816 & 1.912886 & 0.000561 \\
\hline 7 & -4.597697 & -0.708682 & 0.000465 & 7 & 4.522146 & -0.545124 & 0.000328 \\
\hline 1 & -4.625467 & -1.717898 & 0.000286 & 1 & 4.550742 & -1.563112 & 0.000149 \\
\hline 7 & -2.348846 & -2.206907 & -0.000014 & 7 & 2.058570 & -2.131891 & -0.000152 \\
\hline 8 & -1.320737 & -2.943888 & -0.000265 & 8 & 3.127364 & -2.800380 & -0.000119 \\
\hline 7 & 0.197311 & -0.791162 & -0.000574 & 7 & -0.263261 & -0.795400 & -0.000380 \\
\hline 6 & 1.546039 & -0.381546 & -0.000733 & 6 & -1.628973 & -0.446890 & -0.000470 \\
\hline 1 & 0.013611 & -1.805114 & -0.000439 & 1 & -0.061107 & -1.792031 & -0.000255 \\
\hline 6 & 2.503625 & -1.413760 & 0.000721 & 6 & -2.545279 & -1.514983 & 0.000598 \\
\hline 6 & 3.862150 & -1.120461 & -0.000280 & 6 & -3.914725 & -1.275258 & -0.000215 \\
\hline 6 & 4.321980 & 0.206805 & -0.002289 & 6 & -4.426766 & 0.032261 & -0.001753 \\
\hline 6 & 1.987521 & 0.949351 & -0.003774 & 6 & -2.122229 & 0.865243 & -0.002746 \\
\hline 6 & 3.358638 & 1.221530 & -0.004742 & 6 & -3.503127 & 1.083156 & -0.003509 \\
\hline 7 & -2.185141 & 3.186086 & 0.001161 & 7 & 1.966605 & 3.264506 & 0.000871 \\
\hline 1 & 2.173701 & -2.449494 & 0.001591 & 1 & -2.177882 & -2.538549 & 0.001257 \\
\hline 1 & 4.578200 & -1.938755 & -0.000110 & 1 & -4.597455 & -2.121565 & -0.000057 \\
\hline 1 & 1.265082 & 1.751569 & -0.006303 & 1 & -1.431710 & 1.695226 & -0.004623 \\
\hline 1 & 3.680736 & 2.260147 & -0.008090 & 1 & -3.864897 & 2.108641 & -0.005983 \\
\hline 1 & -3.063348 & 3.678764 & 0.002319 & 1 & 2.822076 & 3.795806 & 0.001713 \\
\hline 1 & -1.311859 & 3.686752 & 0.002062 & 1 & 1.071907 & 3.725780 & 0.001534 \\
\hline 1 & 6.317472 & 0.020355 & -0.824565 & 1 & -6.139199 & 1.352617 & -0.061827 \\
\hline 1 & -5.439949 & -0.155579 & 0.000787 & 1 & 5.343770 & 0.041441 & 0.000531 \\
\hline 6 & 5.800648 & 0.516537 & 0.005089 & 6 & -5.916625 & 0.283676 & 0.003694 \\
\hline 1 & 6.275519 & 0.173907 & 0.932539 & 1 & -6.383157 & -0.097646 & 0.920124 \\
\hline 1 & 5.981851 & 1.591606 & -0.083177 & 1 & -6.409607 & -0.212757 & -0.840322 \\
\hline
\end{tabular}

Compound 6g

\begin{tabular}{|c|c|c|c|c|c|c|c|}
\hline & & rotamer $\mathrm{A}$ & & & & rotamer B & \\
\hline Atomic number & $\mathbf{x}$ & $\mathbf{y}$ & $\mathbf{Z}$ & Atomic number & $\mathbf{x}$ & $\mathbf{y}$ & $\mathbf{z}$ \\
\hline 6 & -2.320701 & 0.259939 & 0.000000 & 6 & 2.108542 & -0.776478 & 0.000003 \\
\hline 6 & -0.930405 & -0.152582 & 0.000000 & 6 & 0.849593 & -0.056887 & -0.000002 \\
\hline 6 & -3.290106 & -0.806597 & 0.000000 & 6 & 3.296697 & 0.038134 & 0.000005 \\
\hline 7 & -0.591091 & -1.449151 & 0.000000 & 7 & 0.807950 & 1.275803 & -0.000013 \\
\hline 7 & -2.932312 & -2.091018 & 0.000000 & 7 & 3.234055 & 1.376247 & -0.000008 \\
\hline 6 & -1.604885 & -2.334952 & 0.000000 & 6 & 2.001655 & 1.911922 & -0.000021 \\
\hline 7 & -4.603483 & -0.517815 & 0.000000 & 7 & 4.502935 & -0.543442 & 0.000023 \\
\hline 1 & -4.896548 & 0.448000 & 0.000000 & 1 & 4.532275 & -1.561489 & 0.000062 \\
\hline 7 & -2.826688 & 1.519365 & 0.000000 & 7 & 2.041625 & -2.132820 & 0.000008 \\
\hline 8 & -2.026207 & 2.499686 & 0.000000 & 8 & 3.111245 & -2.800406 & 0.000014 \\
\hline 7 & 0.000000 & 0.822145 & 0.000000 & 7 & -0.281302 & -0.798110 & 0.000003 \\
\hline 6 & 1.409605 & 0.779182 & 0.000000 & 6 & -1.648149 & -0.448607 & 0.000001 \\
\hline 1 & -0.444490 & 1.752433 & 0.000000 & 1 & -0.078860 & -1.794688 & 0.000001 \\
\hline 6 & 2.067251 & 2.024483 & 0.000000 & 6 & -2.567604 & -1.514038 & -0.000056 \\
\hline 6 & 3.453726 & 2.105166 & 0.000000 & 6 & -3.937710 & -1.282392 & -0.000054 \\
\hline 6 & 4.218203 & 0.933038 & 0.000000 & 6 & -4.420887 & 0.030326 & 0.000005 \\
\hline 6 & 2.181942 & -0.391667 & 0.000000 & 6 & -2.138506 & 0.864834 & 0.000061 \\
\hline 6 & 3.576265 & -0.307357 & 0.000000 & 6 & -3.516782 & 1.094462 & 0.000062 \\
\hline 7 & -1.253394 & -3.642063 & 0.000000 & 7 & 1.944505 & 3.263924 & -0.000039 \\
\hline 1 & 1.480396 & 2.939070 & 0.000000 & 1 & -2.203218 & -2.538342 & -0.000103 \\
\hline 1 & 3.955476 & 3.066938 & 0.000000 & 1 & -4.641239 & -2.108119 & -0.000098 \\
\hline 1 & 1.691484 & -1.353484 & 0.000000 & 1 & -1.445582 & 1.692865 & 0.000106 \\
\hline 1 & 4.163012 & -1.223646 & 0.000000 & 1 & -3.882257 & 2.119264 & 0.000109 \\
\hline 1 & -1.972757 & -4.346666 & 0.000000 & 1 & 2.800256 & 3.794754 & -0.000074 \\
\hline 1 & -0.279988 & -3.897994 & 0.000000 & 1 & 1.050284 & 3.725856 & -0.000084 \\
\hline 8 & 5.583133 & 1.068789 & 0.000000 & 8 & -5.782375 & 0.203147 & 0.000004 \\
\hline 1 & 6.001869 & 0.198194 & 0.000000 & 1 & -5.996098 & 1.145219 & 0.000052 \\
\hline 1 & -5.270462 & -1.272811 & 0.000000 & 1 & 5.324018 & 0.043887 & 0.000051 \\
\hline
\end{tabular}

Compound $\mathbf{6 h}$

Compound 6h
\begin{tabular}{|c|c|c|c|c|c|c|c|}
\hline & & rotamer A & & & & rotamer B & \\
\hline Atomic number & $\mathbf{x}$ & $\mathbf{y}$ & $\mathbf{z}$ & Atomic number & $\mathbf{x}$ & $\mathbf{y}$ & $\mathbf{z}$ \\
\hline 6 & 2.179571 & -0.862431 & 0.004412 & 6 & 2.118189 & -0.777474 & 0.004418 \\
\hline 6 & 0.946753 & -0.097302 & -0.001056 & 6 & 0.858123 & -0.057546 & -0.001623 \\
\hline 6 & 3.396197 & -0.089000 & 0.004721 & 6 & 3.306186 & 0.037900 & 0.003723 \\
\hline 7 & 0.962920 & 1.244012 & -0.006035 & 7 & 0.817934 & 1.276281 & -0.007470 \\
\hline 7 & 3.390558 & 1.243919 & -0.001096 & 7 & 3.244133 & 1.375719 & -0.003497 \\
\hline 6 & 2.173438 & 1.829533 & -0.006851 & 6 & 2.010731 & 1.911226 & -0.009428 \\
\hline 7 & 4.586629 & -0.715605 & 0.010467 & 7 & 4.512253 & -0.544674 & 0.009793 \\
\hline
\end{tabular}




\begin{tabular}{|c|c|c|c|c|c|c|c|}
\hline 1 & 4.611674 & -1.724881 & 0.013971 & 1 & 4.539442 & -1.562994 & 0.014694 \\
\hline 7 & 2.335334 & -2.209908 & 0.009498 & 7 & 2.052718 & -2.132946 & 0.010136 \\
\hline 8 & 1.303256 & -2.944628 & 0.008649 & 8 & 3.123423 & -2.800899 & 0.015834 \\
\hline 7 & -0.207054 & -0.790515 & -0.001253 & 7 & -0.272034 & -0.796610 & -0.001291 \\
\hline 6 & -1.556261 & -0.378959 & -0.004356 & 6 & -1.640110 & -0.447386 & -0.004122 \\
\hline 1 & -0.023172 & -1.805154 & 0.000178 & 1 & -0.070426 & -1.793337 & -0.000868 \\
\hline 6 & -2.518747 & -1.404258 & -0.026573 & 6 & -2.560276 & -1.509039 & -0.034246 \\
\hline 6 & -3.877501 & -1.115321 & -0.026370 & 6 & -3.930463 & -1.274470 & -0.033373 \\
\hline 6 & -4.327791 & 0.216600 & -0.002061 & 6 & -4.433973 & 0.037208 & -0.001126 \\
\hline 6 & -1.999088 & 0.953139 & 0.017497 & 6 & -2.135377 & 0.865118 & 0.026108 \\
\hline 6 & -3.363693 & 1.237069 & 0.018296 & 6 & -3.510728 & 1.094038 & 0.026925 \\
\hline 7 & 2.180872 & 3.183761 & -0.010026 & 7 & 1.954752 & 3.263847 & -0.014403 \\
\hline 1 & -2.195139 & -2.441773 & -0.043266 & 1 & -2.198604 & -2.534292 & -0.057780 \\
\hline 1 & -4.597330 & -1.929456 & -0.049934 & 1 & -4.616373 & -2.117193 & -0.063393 \\
\hline 1 & -1.274884 & 1.753898 & 0.035174 & 1 & -1.443785 & 1.694153 & 0.049663 \\
\hline 1 & -3.685176 & 2.275834 & 0.030536 & 1 & -3.872280 & 2.119427 & 0.045293 \\
\hline 1 & 3.060932 & 3.672032 & -0.039937 & 1 & 2.810804 & 3.793293 & -0.043397 \\
\hline 1 & 1.309887 & 3.686906 & -0.046313 & 1 & 1.060879 & 3.725177 & -0.049953 \\
\hline 1 & -5.959099 & 1.424690 & 0.290907 & 1 & -6.111127 & 1.176764 & 0.302124 \\
\hline 1 & 5.429866 & -0.164158 & 0.010175 & 1 & 5.333663 & 0.042111 & 0.009232 \\
\hline 7 & -5.692811 & 0.514273 & -0.058313 & 7 & -5.811292 & 0.281232 & -0.058734 \\
\hline 1 & -6.316017 & -0.214892 & 0.260671 & 1 & -6.402649 & -0.473308 & 0.262433 \\
\hline
\end{tabular}

Compound $\mathbf{6 i}$

\begin{tabular}{|c|c|c|c|c|c|c|c|}
\hline & & rotamer $\mathrm{A}$ & & & & rotamer B & \\
\hline Atomic number & $\mathbf{x}$ & $\mathbf{y}$ & $\mathbf{Z}$ & Atomic number & $\mathbf{x}$ & $\mathbf{y}$ & $\mathbf{Z}$ \\
\hline 6 & 2.940562 & -0.823819 & 0.017026 & 6 & 2.875561 & -0.760507 & 0.015778 \\
\hline 6 & 1.678059 & -0.108885 & -0.007595 & 6 & 1.599320 & -0.069283 & -0.008991 \\
\hline 6 & 4.124934 & -0.001859 & 0.027216 & 6 & 4.044654 & 0.081899 & 0.023688 \\
\hline 7 & 1.640904 & 1.232531 & -0.021676 & 7 & 1.529438 & 1.263740 & -0.024682 \\
\hline 7 & 4.066259 & 1.329565 & 0.012557 & 7 & 3.952603 & 1.417797 & 0.006830 \\
\hline 6 & 2.826336 & 1.865751 & -0.012177 & 6 & 2.707112 & 1.925032 & -0.017442 \\
\hline 7 & 5.339448 & -0.580436 & 0.051653 & 7 & 5.263499 & -0.473310 & 0.047836 \\
\hline 1 & 5.404179 & -1.587881 & 0.062612 & 1 & 5.313194 & -1.490844 & 0.059304 \\
\hline 7 & 3.150470 & -2.163660 & 0.032372 & 7 & 2.841259 & -2.116748 & 0.029131 \\
\hline 8 & 2.148281 & -2.939296 & 0.023606 & 8 & 3.927116 & -2.760291 & 0.051417 \\
\hline 7 & 0.552771 & -0.846719 & -0.016301 & 7 & 0.486170 & -0.832631 & -0.016203 \\
\hline 6 & -0.810965 & -0.488333 & -0.036970 & 6 & -0.888942 & -0.513522 & -0.037852 \\
\hline 1 & 0.776226 & -1.853310 & -0.006510 & 1 & 0.709028 & -1.824799 & -0.007436 \\
\hline 6 & -1.735044 & -1.546169 & -0.063658 & 6 & -1.787648 & -1.590942 & -0.075936 \\
\hline 6 & -3.104249 & -1.315227 & -0.078688 & 6 & -3.162850 & -1.391463 & -0.091615 \\
\hline 6 & -3.624654 & 0.000918 & -0.078053 & 6 & -3.713533 & -0.088699 & -0.081186 \\
\hline 6 & -1.311048 & 0.821980 & -0.019528 & 6 & -1.418459 & 0.784035 & -0.009357 \\
\hline 6 & -2.684692 & 1.055474 & -0.034664 & 6 & -2.797632 & 0.985558 & -0.025872 \\
\hline 7 & 2.779973 & 3.219522 & -0.025386 & 7 & 2.621165 & 3.276268 & -0.033379 \\
\hline 1 & -1.374476 & -2.571740 & -0.067128 & 1 & -1.406434 & -2.609522 & -0.087806 \\
\hline 1 & -3.765701 & -2.172056 & -0.092782 & 1 & -3.803518 & -2.263784 & -0.114633 \\
\hline 1 & -0.622641 & 1.653540 & 0.011046 & 1 & -0.749472 & 1.630982 & 0.030178 \\
\hline 1 & -3.019101 & 2.085176 & -0.012896 & 1 & -3.154824 & 2.007363 & 0.004028 \\
\hline 1 & 3.640101 & 3.742446 & -0.045997 & 1 & 3.465439 & 3.824600 & -0.053579 \\
\hline 1 & 1.889898 & 3.686938 & -0.073592 & 1 & 1.717470 & 3.716976 & -0.079853 \\
\hline 1 & 6.159909 & 0.004237 & 0.058923 & 1 & 6.071360 & 0.131937 & 0.053020 \\
\hline 7 & -4.995307 & 0.242913 & -0.133124 & 7 & -5.091000 & 0.123071 & -0.140037 \\
\hline 6 & -5.916271 & -0.860667 & 0.092655 & 6 & -5.983974 & -1.003426 & 0.084103 \\
\hline 1 & -6.939441 & -0.497034 & -0.015442 & 1 & -7.015909 & -0.665664 & -0.025177 \\
\hline 1 & -5.770462 & -1.650645 & -0.651509 & 1 & -5.817631 & -1.788210 & -0.661107 \\
\hline 1 & -5.811282 & -1.308680 & 1.094547 & 1 & -5.869323 & -1.450552 & 1.085678 \\
\hline 6 & -5.485140 & 1.585850 & 0.136754 & 6 & -5.609923 & 1.448696 & 0.159931 \\
\hline 1 & -5.238999 & 1.935494 & 1.153065 & 1 & -5.373882 & 1.780332 & 1.184887 \\
\hline 1 & -5.072766 & 2.303726 & -0.580044 & 1 & -5.211623 & 2.191507 & -0.539023 \\
\hline 1 & -6.570295 & 1.598055 & 0.022287 & 1 & -6.694857 & 1.440445 & 0.042221 \\
\hline
\end{tabular}

Compound 7a

\begin{tabular}{|c|c|c|c|c|c|c|c|}
\hline & & rotamer A & & & & rotamer B & \\
\hline Atomic number & $\mathbf{x}$ & $\mathbf{y}$ & $\mathbf{z}$ & Atomic number & $\mathbf{x}$ & $\mathbf{y}$ & $\mathbf{z}$ \\
\hline 6 & -1.480329 & -0.750858 & -0.000008 & 6 & 1.416995 & -0.732251 & 0.000085 \\
\hline 6 & -0.215914 & -0.045559 & -0.000005 & 6 & 0.144509 & -0.040310 & 0.000067 \\
\hline 6 & -2.661584 & 0.080775 & -0.000007 & 6 & 2.589565 & 0.112378 & 0.000119 \\
\hline 7 & -0.166626 & 1.288457 & -0.000002 & 7 & 0.069335 & 1.286513 & 0.000072 \\
\hline 7 & -2.595540 & 1.396979 & -0.000004 & 7 & 2.496244 & 1.432401 & 0.000142 \\
\hline 6 & -1.351159 & 1.931143 & -0.000001 & 6 & 1.249596 & 1.947597 & 0.000107 \\
\hline 7 & -3.874491 & -0.567424 & -0.000011 & 7 & 3.807657 & -0.511946 & 0.000140 \\
\hline
\end{tabular}




\begin{tabular}{|c|c|c|c|c|c|c|c|}
\hline 1 & -3.774345 & -1.579296 & -0.000009 & 1 & 3.712725 & -1.535114 & 0.000265 \\
\hline 7 & -1.692971 & -2.094496 & -0.000010 & 7 & 1.353877 & -2.092439 & 0.000033 \\
\hline 8 & -0.701971 & -2.875158 & -0.000010 & 8 & 2.418063 & -2.765018 & 0.000056 \\
\hline 7 & 0.910019 & -0.798478 & -0.000005 & 7 & -0.975091 & -0.812056 & 0.000038 \\
\hline 6 & 2.268736 & -0.450307 & -0.000002 & 6 & -2.340024 & -0.494783 & 0.000007 \\
\hline 1 & 0.679327 & -1.803174 & -0.000007 & 1 & -0.746648 & -1.803606 & 0.000024 \\
\hline 6 & 3.179460 & -1.526578 & 0.000000 & 6 & -3.232838 & -1.585562 & -0.000041 \\
\hline 6 & 4.547230 & -1.299369 & 0.000003 & 6 & -4.604390 & -1.381658 & -0.000069 \\
\hline 6 & 5.042668 & 0.017753 & 0.000004 & 6 & -5.122685 & -0.073637 & -0.000051 \\
\hline 6 & 2.761894 & 0.866736 & -0.000001 & 6 & -2.855652 & 0.813570 & 0.000026 \\
\hline 6 & 4.135622 & 1.090073 & 0.000002 & 6 & -4.232799 & 1.013162 & -0.000003 \\
\hline 7 & -1.309208 & 3.277310 & 0.000003 & 7 & 1.180955 & 3.292384 & 0.000118 \\
\hline 1 & 2.804435 & -2.546019 & -0.000001 & 1 & -2.843115 & -2.599926 & -0.000055 \\
\hline 1 & 5.237426 & -2.136095 & 0.000004 & 1 & -5.279779 & -2.230403 & -0.000106 \\
\hline 1 & 2.069964 & 1.694869 & -0.000002 & 1 & -2.177741 & 1.653282 & 0.000061 \\
\hline 1 & 4.511966 & 2.107686 & 0.000003 & 1 & -4.625633 & 2.024535 & 0.000011 \\
\hline 1 & -2.176983 & 3.791181 & 0.000003 & 1 & 2.038318 & 3.823578 & 0.000106 \\
\hline 1 & -0.423543 & 3.756880 & 0.000005 & 1 & 0.285789 & 3.754008 & 0.000077 \\
\hline 6 & -5.179237 & -0.041560 & -0.000004 & 6 & 5.102670 & 0.047724 & -0.000153 \\
\hline 8 & -5.436787 & 1.143453 & -0.000014 & 8 & 5.332915 & 1.238218 & -0.000491 \\
\hline 6 & -6.238964 & -1.130559 & 0.000037 & 6 & 6.184419 & -1.017471 & -0.000016 \\
\hline 1 & -6.143532 & -1.769260 & -0.885285 & 1 & 6.098801 & -1.658544 & 0.884416 \\
\hline 1 & -7.220811 & -0.658323 & -0.000147 & 1 & 7.157073 & -0.526468 & -0.000191 \\
\hline 1 & -6.143730 & -1.768956 & 0.885601 & 1 & 6.098671 & -1.658898 & -0.884179 \\
\hline 6 & 6.454613 & 0.261821 & 0.000007 & 6 & -6.538364 & 0.146307 & -0.000080 \\
\hline 7 & 7.601914 & 0.460949 & 0.000010 & 7 & -7.689016 & 0.325367 & -0.000104 \\
\hline
\end{tabular}

Compound $7 \mathbf{b}$

\begin{tabular}{|c|c|c|c|c|c|c|c|}
\hline & & rotamer A & & & & rotamer B & \\
\hline Atomic number & $\mathbf{x}$ & $\mathbf{y}$ & $\mathbf{Z}$ & Atomic number & $\mathbf{x}$ & $\mathbf{y}$ & $\mathbf{z}$ \\
\hline 6 & 2.276373 & -0.756540 & 0.000002 & 6 & -2.219916 & -0.739340 & -0.004714 \\
\hline 6 & 1.004440 & -0.063544 & 0.000003 & 6 & -0.940582 & -0.058228 & -0.010314 \\
\hline 6 & 3.449389 & 0.086379 & 0.000001 & 6 & -3.385109 & 0.115382 & 0.000977 \\
\hline 7 & 0.943468 & 1.270764 & 0.000005 & 7 & -0.855665 & 1.268742 & -0.010396 \\
\hline 7 & 3.371461 & 1.401754 & 0.000003 & 7 & -3.281360 & 1.434456 & 0.000923 \\
\hline 6 & 2.121381 & 1.923847 & 0.000006 & 6 & -2.029838 & 1.938864 & -0.004649 \\
\hline 7 & 4.668539 & -0.551095 & -0.000002 & 7 & -4.608570 & -0.499245 & 0.006750 \\
\hline 1 & 4.576643 & -1.563791 & -0.000002 & 1 & -4.521170 & -1.523342 & 0.006230 \\
\hline 7 & 2.502332 & -2.097662 & 0.000002 & 7 & -2.169164 & -2.099551 & -0.005280 \\
\hline 8 & 1.519142 & -2.888454 & 0.000003 & 8 & -3.239609 & -2.763176 & -0.000521 \\
\hline 7 & -0.113390 & -0.825648 & 0.000002 & 7 & 0.171773 & -0.837427 & -0.015263 \\
\hline 6 & -1.477506 & -0.489320 & 0.000001 & 6 & 1.541420 & -0.529874 & -0.020456 \\
\hline 1 & 0.125113 & -1.828053 & 0.000003 & 1 & -0.062869 & -1.827322 & -0.013224 \\
\hline 6 & -2.376846 & -1.575167 & 0.000009 & 6 & 2.425293 & -1.626226 & -0.018037 \\
\hline 6 & -3.746704 & -1.358616 & 0.000006 & 6 & 3.800116 & -1.430043 & -0.024303 \\
\hline 6 & -4.250443 & -0.050597 & -0.000005 & 6 & 4.321789 & -0.131228 & -0.035837 \\
\hline 6 & -1.984300 & 0.819757 & -0.000010 & 6 & 2.067118 & 0.773006 & -0.031054 \\
\hline 6 & -3.364565 & 1.027348 & -0.000012 & 6 & 3.448346 & 0.959342 & -0.037196 \\
\hline 7 & 2.067396 & 3.269971 & 0.000011 & 7 & -1.950565 & 3.283408 & -0.004417 \\
\hline 1 & -1.991758 & -2.590880 & 0.000017 & 1 & 2.028686 & -2.637966 & -0.013667 \\
\hline 1 & -4.425253 & -2.205531 & 0.000012 & 1 & 4.465785 & -2.286243 & -0.026505 \\
\hline 1 & -1.302617 & 1.656289 & -0.000017 & 1 & 1.396972 & 1.618823 & -0.036570 \\
\hline 1 & -3.747506 & 2.041723 & -0.000021 & 1 & 3.845474 & 1.968730 & -0.049353 \\
\hline 1 & 2.930455 & 3.791585 & 0.000014 & 1 & -2.803602 & 3.821364 & 0.000628 \\
\hline 1 & 1.177212 & 3.741046 & 0.000017 & 1 & -1.051536 & 3.737401 & -0.007168 \\
\hline 6 & 5.968202 & -0.014659 & -0.000005 & 6 & -5.898607 & 0.070103 & 0.013774 \\
\hline 8 & 6.216888 & 1.172449 & -0.000006 & 8 & -6.120762 & 1.262333 & 0.014357 \\
\hline 6 & 7.037042 & -1.095041 & -0.000008 & 6 & -6.988585 & -0.986951 & 0.020971 \\
\hline 1 & 6.946975 & -1.734403 & 0.885409 & 1 & -6.911289 & -1.631675 & -0.861563 \\
\hline 1 & 8.014985 & -0.614752 & -0.000017 & 1 & -7.957483 & -0.488553 & 0.023404 \\
\hline 1 & 6.946961 & -1.734414 & -0.885415 & 1 & -6.904205 & -1.626084 & 0.906936 \\
\hline 6 & -5.738509 & 0.156748 & -0.000003 & 6 & 5.807072 & 0.087362 & 0.011893 \\
\hline 9 & -6.085160 & 1.466063 & -0.000052 & 9 & 6.177466 & 1.245959 & -0.589887 \\
\hline 9 & -6.331069 & -0.409152 & 1.087283 & 9 & 6.495356 & -0.914562 & -0.591808 \\
\hline 9 & -6.331084 & -0.409239 & -1.087234 & 9 & 6.274060 & 0.154816 & 1.291429 \\
\hline
\end{tabular}

Compound 7c

\begin{tabular}{|c|c|c|c|c|c|c|c|}
\hline & & rotamer A & & & & rotamer B & \\
\hline Atomic number & $\mathbf{x}$ & $\mathbf{y}$ & $\mathbf{z}$ & Atomic number & $\mathbf{x}$ & $\mathbf{y}$ & $\mathbf{z}$ \\
\hline 6 & -2.289240 & -0.757296 & -0.000116 & 6 & -2.229824 & -0.736889 & -0.000005 \\
\hline 6 & -1.030824 & -0.039227 & -0.000099 & 6 & -0.967126 & -0.023950 & -0.000005 \\
\hline 6 & -3.478586 & 0.062595 & -0.000092 & 6 & -3.415818 & 0.089128 & -0.000003 \\
\hline
\end{tabular}




\begin{tabular}{|c|c|c|c|c|c|c|c|}
\hline 7 & -0.997669 & 1.296307 & -0.000073 & 7 & -0.916345 & 1.304972 & -0.000005 \\
\hline 7 & -3.427615 & 1.379000 & -0.000062 & 7 & -3.345518 & 1.410103 & -0.000002 \\
\hline 6 & -2.187391 & 1.925633 & -0.000056 & 6 & -2.106078 & 1.945273 & -0.000003 \\
\hline 7 & -4.685039 & -0.599764 & -0.000103 & 7 & -4.623692 & -0.556507 & -0.000001 \\
\hline 1 & -4.571660 & -1.610313 & -0.000097 & 1 & -4.509397 & -1.578208 & -0.000003 \\
\hline 7 & -2.489847 & -2.102347 & -0.000134 & 7 & -2.146446 & -2.095058 & -0.000006 \\
\hline 8 & -1.491829 & -2.874633 & -0.000152 & 8 & -3.201010 & -2.784845 & -0.000005 \\
\hline 7 & 0.102374 & -0.777704 & -0.000107 & 7 & 0.164093 & -0.774284 & -0.000006 \\
\hline 6 & 1.459892 & -0.415433 & -0.000071 & 6 & 1.526366 & -0.435117 & -0.000005 \\
\hline 1 & -0.114723 & -1.784548 & -0.000128 & 1 & -0.045451 & -1.769743 & -0.000007 \\
\hline 6 & 2.379136 & -1.483893 & -0.000052 & 6 & 2.433614 & -1.513378 & -0.000003 \\
\hline 6 & 3.745924 & -1.242617 & -0.000009 & 6 & 3.803227 & -1.288072 & -0.000001 \\
\hline 6 & 4.231244 & 0.075146 & 0.000014 & 6 & 4.304206 & 0.023561 & 0.000000 \\
\hline 6 & 1.941598 & 0.905782 & -0.000051 & 6 & 2.023348 & 0.880283 & -0.000004 \\
\hline 6 & 3.314736 & 1.134915 & -0.000010 & 6 & 3.398897 & 1.093128 & -0.000002 \\
\hline 7 & -2.160018 & 3.272762 & -0.000028 & 7 & -2.060246 & 3.291484 & -0.000003 \\
\hline 1 & 2.011005 & -2.506196 & -0.000067 & 1 & 2.056026 & -2.532730 & -0.000003 \\
\hline 1 & 4.441874 & -2.073274 & 0.000008 & 1 & 4.489147 & -2.127058 & 0.000001 \\
\hline 1 & 1.243734 & 1.728835 & -0.000067 & 1 & 1.334915 & 1.711329 & -0.000006 \\
\hline 1 & 3.695608 & 2.150796 & 0.000007 & 1 & 3.790869 & 2.104800 & -0.000002 \\
\hline 1 & -3.032943 & 3.777523 & -0.000021 & 1 & -2.926135 & 3.808389 & -0.000002 \\
\hline 1 & -1.278837 & 3.760607 & -0.000026 & 1 & -1.172270 & 3.766921 & -0.000004 \\
\hline 6 & -5.994898 & -0.090844 & 0.000068 & 6 & -5.927299 & -0.020882 & 0.000007 \\
\hline 8 & -6.269431 & 1.090766 & 0.000047 & 8 & -6.181190 & 1.165214 & 0.000011 \\
\hline 6 & -7.041008 & -1.193643 & 0.000440 & 6 & -6.989807 & -1.105938 & 0.000013 \\
\hline 1 & -6.937310 & -1.831497 & -0.884563 & 1 & -6.892369 & -1.745532 & -0.884255 \\
\hline 1 & -8.028821 & -0.733968 & 0.000047 & 1 & -7.971280 & -0.632736 & 0.000052 \\
\hline 1 & -6.937579 & -1.830437 & 0.886246 & 1 & -6.892318 & -1.745576 & 0.884243 \\
\hline 8 & 6.142164 & 1.524136 & 0.000079 & 8 & 6.231265 & 1.450399 & 0.000002 \\
\hline 8 & 6.460942 & -0.713404 & 0.000098 & 8 & 6.524372 & -0.790793 & 0.000006 \\
\hline 6 & 5.683233 & 0.395353 & 0.000065 & 6 & 5.759058 & 0.327029 & 0.000003 \\
\hline 6 & 7.881431 & -0.481146 & 0.000154 & 6 & 7.947309 & -0.574367 & 0.000010 \\
\hline 1 & 8.336270 & -1.471417 & 0.000165 & 1 & 8.391161 & -1.569633 & 0.000016 \\
\hline 1 & 8.175952 & 0.079628 & -0.890166 & 1 & 8.248130 & -0.016925 & -0.890297 \\
\hline 1 & 8.175882 & 0.079614 & 0.890507 & 1 & 8.248124 & -0.016917 & 0.890315 \\
\hline
\end{tabular}

Compound 7d

\begin{tabular}{|c|c|c|c|c|c|c|c|}
\hline & & rotamer $\mathrm{A}$ & & & & rotamer B & \\
\hline Atomic number & $\mathbf{x}$ & $\mathbf{y}$ & $\mathbf{z}$ & Atomic number & $\mathbf{x}$ & $\mathbf{y}$ & $\mathbf{z}$ \\
\hline 6 & -1.628820 & -0.753484 & 0.000022 & 6 & -1.567464 & -0.734981 & 0.000111 \\
\hline 6 & -0.360666 & -0.051577 & 0.000033 & 6 & -0.290836 & -0.045395 & 0.000082 \\
\hline 6 & -2.807412 & 0.081468 & 0.000008 & 6 & -2.737818 & 0.112689 & 0.000148 \\
\hline 7 & -0.310924 & 1.285292 & 0.000037 & 7 & -0.216624 & 1.284130 & 0.000089 \\
\hline 7 & -2.739846 & 1.396976 & 0.000010 & 7 & -2.643711 & 1.432162 & 0.000177 \\
\hline 6 & -1.492386 & 1.928160 & 0.000027 & 6 & -1.394406 & 1.944649 & 0.000137 \\
\hline 7 & -4.022137 & -0.565984 & -0.000011 & 7 & -3.957068 & -0.511463 & 0.000174 \\
\hline 1 & -3.920864 & -1.577863 & -0.000001 & 1 & -3.860126 & -1.535193 & 0.000320 \\
\hline 7 & -1.845015 & -2.095175 & 0.000025 & 7 & -1.509358 & -2.093739 & 0.000056 \\
\hline 8 & -0.854708 & -2.878865 & 0.000036 & 8 & -2.576639 & -2.764804 & 0.000088 \\
\hline 7 & 0.761022 & -0.802591 & 0.000037 & 7 & 0.824891 & -0.813480 & 0.000042 \\
\hline 6 & 2.125216 & -0.454152 & 0.000025 & 6 & 2.195884 & -0.494347 & 0.000003 \\
\hline 1 & 0.531116 & -1.806925 & 0.000039 & 1 & 0.599238 & -1.805263 & 0.000032 \\
\hline 6 & 3.034747 & -1.528050 & 0.000010 & 6 & 3.088481 & -1.581612 & -0.000026 \\
\hline 6 & 4.407259 & -1.303011 & -0.000011 & 6 & 4.464863 & -1.378399 & -0.000058 \\
\hline 6 & 4.884722 & 0.008793 & -0.000017 & 6 & 4.963506 & -0.074938 & -0.000059 \\
\hline 6 & 2.621000 & 0.859613 & 0.000025 & 6 & 2.712434 & 0.811084 & -0.000005 \\
\hline 6 & 3.998777 & 1.084195 & 0.000003 & 6 & 4.093696 & 1.013518 & -0.000036 \\
\hline 7 & -1.450767 & 3.275540 & 0.000035 & 7 & -1.326379 & 3.290501 & 0.000161 \\
\hline 1 & 2.662244 & -2.548704 & 0.000013 & 1 & 2.702477 & -2.597712 & -0.000022 \\
\hline 1 & 5.098926 & -2.137948 & -0.000023 & 1 & 5.142690 & -2.224652 & -0.000080 \\
\hline 1 & 1.932353 & 1.690842 & 0.000040 & 1 & 2.036960 & 1.653112 & 0.000010 \\
\hline 1 & 4.379091 & 2.099837 & 0.000003 & 1 & 4.489308 & 2.023336 & -0.000043 \\
\hline 1 & -2.318533 & 3.789017 & 0.000029 & 1 & -2.183906 & 3.821077 & 0.000160 \\
\hline 1 & -0.564852 & 3.754375 & 0.000053 & 1 & -0.431060 & 3.751579 & 0.000133 \\
\hline 6 & -5.325398 & -0.041204 & -0.000047 & 6 & -5.250821 & 0.046679 & -0.000192 \\
\hline 8 & -5.585753 & 1.143721 & -0.000073 & 8 & -5.484428 & 1.237055 & -0.000554 \\
\hline 6 & -6.385132 & -1.130996 & -0.000043 & 6 & -6.332122 & -1.019754 & -0.000084 \\
\hline 1 & -6.289468 & -1.769669 & -0.885365 & 1 & -6.245522 & -1.661390 & -0.884008 \\
\hline 1 & -7.367185 & -0.659117 & -0.000162 & 1 & -7.305231 & -0.529580 & -0.000536 \\
\hline 1 & -6.289609 & -1.769501 & 0.885417 & 1 & -6.246003 & -1.660688 & 0.884398 \\
\hline 17 & 6.617218 & 0.303522 & -0.000053 & 17 & 6.700887 & 0.192672 & -0.000097 \\
\hline
\end{tabular}


Compound 7e

\begin{tabular}{|c|c|c|c|c|c|c|c|}
\hline & & rotamer A & & & & rotamer B & \\
\hline Atomic number & $\mathbf{x}$ & $\mathbf{y}$ & $\mathbf{Z}$ & Atomic number & $\mathbf{x}$ & $\mathbf{y}$ & $\mathbf{z}$ \\
\hline 6 & 0.223359 & 0.007680 & 0.055173 & 6 & -0.794839 & -0.725330 & -0.000001 \\
\hline 6 & 0.134289 & 0.000936 & 1.502732 & 6 & 0.469511 & -0.010864 & 0.000000 \\
\hline 6 & 1.560181 & -0.009609 & -0.491180 & 6 & -1.981421 & 0.099613 & -0.000001 \\
\hline 7 & 1.237429 & -0.020093 & 2.260713 & 7 & 0.515506 & 1.320752 & 0.000002 \\
\hline 7 & 2.634345 & -0.030218 & 0.270533 & 7 & -1.914162 & 1.420423 & 0.000002 \\
\hline 6 & 2.412365 & -0.034326 & 1.608729 & 6 & -0.674182 & 1.956827 & 0.000003 \\
\hline 7 & 1.664822 & -0.003519 & -1.864395 & 7 & -3.188484 & -0.549031 & -0.000002 \\
\hline 1 & 0.755772 & 0.012869 & -2.320045 & 1 & -3.070002 & -1.570860 & 0.000000 \\
\hline 7 & -0.791315 & 0.028179 & -0.848581 & 7 & -0.711796 & -2.082263 & -0.000002 \\
\hline 8 & -1.984664 & 0.044030 & -0.435650 & 8 & -1.766936 & -2.773694 & -0.000003 \\
\hline 7 & -1.099553 & 0.016669 & 2.046959 & 7 & 1.599178 & -0.755290 & -0.000001 \\
\hline 6 & -1.541816 & 0.015907 & 3.386224 & 6 & 2.967003 & -0.412997 & 0.000000 \\
\hline 1 & -1.824419 & 0.031791 & 1.315939 & 1 & 1.393858 & -1.751360 & -0.000002 \\
\hline 6 & -2.937619 & 0.036130 & 3.568242 & 6 & 3.873023 & -1.489706 & -0.000003 \\
\hline 6 & -3.483110 & 0.037268 & 4.848733 & 6 & 5.245944 & -1.258940 & -0.000003 \\
\hline 6 & -2.648481 & 0.018289 & 5.970896 & 6 & 5.741588 & 0.048580 & 0.000001 \\
\hline 6 & -0.700948 & -0.003181 & 4.510915 & 6 & 3.460877 & 0.901334 & 0.000003 \\
\hline 6 & -1.264444 & -0.001757 & 5.789386 & 6 & 4.841489 & 1.115698 & 0.000004 \\
\hline 7 & 3.527774 & -0.055540 & 2.366480 & 7 & -0.633297 & 3.304289 & 0.000005 \\
\hline 1 & -3.590293 & 0.050924 & 2.699308 & 1 & 3.495649 & -2.509452 & -0.000006 \\
\hline 1 & -4.562310 & 0.053022 & 4.968418 & 1 & 5.927968 & -2.104017 & -0.000005 \\
\hline 1 & -3.072379 & 0.019149 & 6.970330 & 1 & 6.811759 & 0.230436 & 0.000001 \\
\hline 1 & 0.369874 & -0.018723 & 4.376286 & 1 & 2.770038 & 1.730750 & 0.000006 \\
\hline 1 & -0.605046 & -0.016621 & 6.652582 & 1 & 5.212138 & 2.136859 & 0.000006 \\
\hline 1 & 4.426375 & -0.066661 & 1.909497 & 1 & -1.501100 & 3.817675 & 0.000004 \\
\hline 1 & 3.455865 & -0.060066 & 3.370906 & 1 & 0.253104 & 3.782252 & 0.000005 \\
\hline 6 & 2.804729 & -0.016284 & -2.683856 & 6 & -4.492456 & -0.017733 & -0.000007 \\
\hline 8 & 3.945621 & -0.035972 & -2.270925 & 8 & -4.751739 & 1.167621 & -0.000025 \\
\hline 6 & 2.451000 & -0.002596 & -4.162533 & 6 & -5.551988 & -1.106242 & 0.000021 \\
\hline 1 & 1.846756 & -0.877317 & -4.428197 & 1 & -5.452437 & -1.745719 & -0.884086 \\
\hline 1 & 3.374950 & -0.013559 & -4.739893 & 1 & -6.534936 & -0.636040 & -0.000084 \\
\hline 1 & 1.873962 & 0.893195 & -4.418293 & 1 & -5.452549 & -1.745541 & 0.884270 \\
\hline
\end{tabular}

Compound 7f

\begin{tabular}{|c|c|c|c|c|c|c|c|}
\hline & & rotamer A & & & & rotamer B & \\
\hline Atomic number & $\mathbf{x}$ & $\mathbf{y}$ & $\mathbf{Z}$ & Atomic number & $\mathbf{x}$ & $\mathbf{y}$ & $\mathbf{Z}$ \\
\hline 6 & 1.266219 & -0.748325 & 0.000265 & 6 & 1.203161 & -0.730882 & -0.000023 \\
\hline 6 & 0.004529 & -0.032513 & 0.000287 & 6 & -0.070122 & -0.031270 & -0.000063 \\
\hline 6 & 2.453660 & 0.073709 & 0.000285 & 6 & 2.379727 & 0.108366 & 0.000152 \\
\hline 7 & -0.027888 & 1.306603 & 0.000605 & 7 & -0.131421 & 1.300551 & 0.000181 \\
\hline 7 & 2.402294 & 1.389497 & 0.000582 & 7 & 2.297113 & 1.428148 & 0.000404 \\
\hline 6 & 1.159422 & 1.934348 & 0.000861 & 6 & 1.050331 & 1.949858 & 0.000477 \\
\hline 7 & 3.661343 & -0.589010 & -0.000032 & 7 & 3.594325 & -0.526606 & 0.000056 \\
\hline 1 & 3.545788 & -1.599457 & -0.000239 & 1 & 3.486758 & -1.549872 & -0.000105 \\
\hline 7 & 1.469020 & -2.091573 & 0.000118 & 7 & 1.136757 & -2.088228 & -0.000180 \\
\hline 8 & 0.470522 & -2.865730 & 0.000116 & 8 & 2.200514 & -2.767390 & -0.000212 \\
\hline 7 & -1.124737 & -0.767688 & -0.000008 & 7 & -1.190485 & -0.787172 & -0.000326 \\
\hline 6 & -2.487830 & -0.404133 & -0.000262 & 6 & -2.562344 & -0.458070 & -0.000358 \\
\hline 1 & -0.909108 & -1.774480 & 0.000122 & 1 & -0.975248 & -1.781089 & -0.000228 \\
\hline 6 & -3.408189 & -1.469329 & 0.001100 & 6 & -3.461101 & -1.540656 & 0.000809 \\
\hline 6 & -4.775945 & -1.223230 & 0.000049 & 6 & -4.834021 & -1.322357 & 0.000277 \\
\hline 6 & -5.281365 & 0.087378 & -0.002074 & 6 & -5.366137 & -0.022821 & -0.001204 \\
\hline 6 & -2.974461 & 0.910767 & -0.003073 & 6 & -3.075180 & 0.846274 & -0.002353 \\
\hline 6 & -4.354018 & 1.135231 & -0.004087 & 6 & -4.459231 & 1.042600 & -0.002833 \\
\hline 7 & 1.134792 & 3.283110 & 0.001403 & 7 & 0.994593 & 3.297090 & 0.000867 \\
\hline 1 & -3.043113 & -2.493172 & 0.002201 & 1 & -3.078004 & -2.558430 & 0.001623 \\
\hline 1 & -5.463257 & -2.065686 & 0.000406 & 1 & -5.503535 & -2.179055 & 0.000760 \\
\hline 1 & -2.281070 & 1.738156 & -0.005155 & 1 & -2.398515 & 1.687480 & -0.003964 \\
\hline 1 & -4.711873 & 2.161962 & -0.007016 & 1 & -4.836869 & 2.062228 & -0.004839 \\
\hline 1 & 2.008691 & 3.785735 & 0.001799 & 1 & 1.856680 & 3.819930 & 0.001225 \\
\hline 1 & 0.254361 & 3.771804 & 0.001983 & 1 & 0.102892 & 3.765013 & 0.001177 \\
\hline 6 & 4.970173 & -0.082344 & -0.000177 & 6 & 4.891970 & 0.018806 & 0.000122 \\
\hline 8 & 5.248541 & 1.098926 & 0.000136 & 8 & 5.138884 & 1.206938 & 0.000344 \\
\hline 6 & 6.015354 & -1.186785 & -0.000833 & 6 & 5.963417 & -1.058170 & -0.000135 \\
\hline 1 & 5.911063 & -1.824600 & 0.884132 & 1 & 5.871001 & -1.698735 & 0.883970 \\
\hline 1 & 7.003766 & -0.728308 & -0.000609 & 1 & 6.941199 & -0.577308 & -0.000258 \\
\hline 1 & 5.910986 & -1.823522 & -0.886568 & 1 & 5.870709 & -1.698617 & -0.884295 \\
\hline 6 & -6.769754 & 0.345193 & 0.003023 & 6 & -6.859638 & 0.204898 & 0.002533 \\
\hline 1 & -7.263819 & -0.149964 & -0.840997 & 1 & -7.340464 & -0.281535 & -0.854236 \\
\hline
\end{tabular}




\begin{tabular}{|c|c|c|c|c|c|c|c|}
\hline 1 & -6.988397 & 1.414849 & -0.062271 & 1 & -7.099259 & 1.271169 & -0.041637 \\
\hline 1 & -7.237060 & -0.035246 & 0.919337 & 1 & -7.323673 & -0.203509 & 0.908328 \\
\hline
\end{tabular}

Compound $7 \mathrm{~g}$

\begin{tabular}{|c|c|c|c|c|c|c|c|}
\hline & & rotamer $\mathrm{A}$ & & & & rotamer B & \\
\hline Atomic number & $\mathbf{x}$ & $\mathbf{y}$ & $\mathbf{z}$ & Atomic number & $\mathbf{x}$ & $\mathbf{y}$ & $\mathbf{z}$ \\
\hline 6 & -1.249580 & -0.749893 & -0.000001 & 6 & 1.187435 & -0.733100 & 0.000074 \\
\hline 6 & 0.012230 & -0.034318 & -0.000001 & 6 & -0.086975 & -0.035066 & 0.000091 \\
\hline 6 & -2.436762 & 0.072362 & -0.000001 & 6 & 2.362716 & 0.107729 & 0.000040 \\
\hline 7 & 0.045188 & 1.306139 & 0.000000 & 7 & -0.149958 & 1.297890 & 0.000098 \\
\hline 7 & -2.385021 & 1.388078 & 0.000000 & 7 & 2.278411 & 1.427445 & 0.000058 \\
\hline 6 & -1.142001 & 1.933191 & 0.000000 & 6 & 1.031021 & 1.947894 & 0.000090 \\
\hline 7 & -3.644386 & -0.590346 & -0.000001 & 7 & 3.578030 & -0.525762 & -0.000027 \\
\hline 1 & -3.528711 & -1.600810 & 0.000001 & 1 & 3.471516 & -1.549251 & -0.000044 \\
\hline 7 & -1.451106 & -2.092724 & -0.000001 & 7 & 1.122690 & -2.090178 & 0.000067 \\
\hline 8 & -0.450498 & -2.865434 & -0.000002 & 8 & 2.187335 & -2.768435 & 0.000032 \\
\hline 7 & 1.140331 & -0.768930 & -0.000001 & 7 & -1.205740 & -0.790733 & 0.000090 \\
\hline 6 & 2.503539 & -0.404376 & 0.000000 & 6 & -2.578564 & -0.460613 & 0.000043 \\
\hline 1 & 0.923393 & -1.776092 & -0.000002 & 1 & -0.990272 & -1.784613 & 0.000088 \\
\hline 6 & 3.427376 & -1.467095 & -0.000005 & 6 & -3.480673 & -1.540567 & 0.000031 \\
\hline 6 & 4.795452 & -1.229270 & -0.000004 & 6 & -4.853975 & -1.330339 & -0.000029 \\
\hline 6 & 5.272184 & 0.086663 & 0.000001 & 6 & -5.357435 & -0.025024 & -0.000079 \\
\hline 6 & 2.987511 & 0.911978 & 0.000005 & 6 & -3.088576 & 0.845161 & -0.000002 \\
\hline 6 & 4.364058 & 1.148101 & 0.000005 & 6 & -4.470018 & 1.053355 & -0.000063 \\
\hline 7 & -1.119381 & 3.282412 & 0.000001 & 7 & 0.975462 & 3.295515 & 0.000113 \\
\hline 1 & 3.065019 & -2.491605 & -0.000009 & 1 & -3.100605 & -2.559136 & 0.000067 \\
\hline 1 & 5.503551 & -2.050993 & -0.000007 & 1 & -5.544729 & -2.166715 & -0.000040 \\
\hline 1 & 2.291605 & 1.737330 & 0.000008 & 1 & -2.409377 & 1.684368 & 0.000008 \\
\hline 1 & 4.725876 & 2.174089 & 0.000010 & 1 & -4.851304 & 2.072253 & -0.000100 \\
\hline 1 & -1.994293 & 3.783245 & -0.000001 & 1 & 1.837902 & 3.817757 & 0.000088 \\
\hline 1 & -0.240181 & 3.773058 & -0.000001 & 1 & 0.084306 & 3.764211 & 0.000126 \\
\hline 6 & -4.953183 & -0.083652 & -0.000003 & 6 & 4.874937 & 0.021359 & -0.000166 \\
\hline 8 & -5.231389 & 1.097678 & -0.000013 & 8 & 5.120205 & 1.209856 & -0.000254 \\
\hline 6 & -5.998461 & -1.187963 & 0.000014 & 6 & 5.947894 & -1.054072 & -0.000048 \\
\hline 1 & -5.894164 & -1.825290 & -0.885300 & 1 & 5.857141 & -1.693564 & 0.885007 \\
\hline 1 & -6.986837 & -0.729407 & -0.000021 & 1 & 6.924993 & -0.571814 & -0.001555 \\
\hline 1 & -5.894198 & -1.825221 & 0.885382 & 1 & 5.855398 & -1.695849 & -0.883252 \\
\hline 8 & 6.631000 & 0.264916 & 0.000002 & 8 & -6.720455 & 0.125908 & -0.000141 \\
\hline 1 & 6.842494 & 1.207640 & 0.000005 & 1 & -6.950637 & 1.064169 & -0.000178 \\
\hline
\end{tabular}

Compound 7h

\begin{tabular}{|c|c|c|c|c|c|c|c|}
\hline & & rotamer A & & & & rotamer B & \\
\hline Atomic number & $\mathbf{x}$ & $\mathbf{y}$ & $\mathbf{z}$ & Atomic number & $\mathbf{x}$ & $\mathbf{y}$ & $\mathbf{z}$ \\
\hline 6 & 1.257775 & -0.749331 & -0.000506 & 6 & -1.195697 & -0.733068 & -0.000056 \\
\hline 6 & -0.004749 & -0.033808 & -0.004347 & 6 & 0.079901 & -0.034840 & -0.004357 \\
\hline 6 & 2.444562 & 0.073784 & 0.000104 & 6 & -2.370655 & 0.108812 & -0.000599 \\
\hline 7 & -0.036793 & 1.308051 & -0.008240 & 7 & 0.141657 & 1.299392 & -0.009295 \\
\hline 7 & 2.393423 & 1.389006 & -0.004245 & 7 & -2.286878 & 1.428083 & -0.006436 \\
\hline 6 & 1.149108 & 1.934142 & -0.009519 & 6 & -1.038217 & 1.948333 & -0.011894 \\
\hline 7 & 3.652340 & -0.590139 & 0.005210 & 7 & -3.585976 & -0.525788 & 0.004960 \\
\hline 1 & 3.534547 & -1.600440 & 0.007580 & 1 & -3.477313 & -1.549515 & 0.008301 \\
\hline 7 & 1.459789 & -2.091198 & 0.003630 & 7 & -1.132689 & -2.089215 & 0.004897 \\
\hline 8 & 0.458069 & -2.864526 & 0.003044 & 8 & -2.198678 & -2.767685 & 0.009510 \\
\hline 7 & -1.132190 & -0.766872 & -0.003746 & 7 & 1.197820 & -0.788456 & -0.003009 \\
\hline 6 & -2.495879 & -0.403297 & -0.004736 & 6 & 2.571822 & -0.459076 & -0.004035 \\
\hline 1 & -0.914878 & -1.774088 & -0.003122 & 1 & 0.982904 & -1.782466 & -0.003097 \\
\hline 6 & -3.420432 & -1.462899 & -0.023726 & 6 & 3.474546 & -1.535530 & -0.031819 \\
\hline 6 & -4.788322 & -1.223010 & -0.021495 & 6 & 4.847975 & -1.322903 & -0.029460 \\
\hline 6 & -5.286197 & 0.092333 & 0.001618 & 6 & 5.372146 & -0.018933 & 0.001917 \\
\hline 6 & -2.985656 & 0.912308 & 0.015952 & 6 & 3.087117 & 0.845642 & 0.025508 \\
\hline 6 & -4.359081 & 1.147209 & 0.018843 & 6 & 4.465554 & 1.052801 & 0.027897 \\
\hline 7 & 1.128025 & 3.284021 & -0.015685 & 7 & -0.983974 & 3.296462 & -0.019841 \\
\hline 1 & -3.060363 & -2.488321 & -0.039538 & 1 & 3.096927 & -2.555011 & -0.054767 \\
\hline 1 & -5.478497 & -2.062391 & -0.042623 & 1 & 5.520471 & -2.176312 & -0.057700 \\
\hline 1 & -2.291351 & 1.739106 & 0.031009 & 1 & 2.409453 & 1.686037 & 0.047233 \\
\hline 1 & -4.717391 & 2.173716 & 0.030092 & 1 & 4.843214 & 2.072254 & 0.045685 \\
\hline 1 & 2.003334 & 3.783856 & -0.023918 & 1 & -1.846780 & 3.817827 & -0.029150 \\
\hline 1 & 0.249069 & 3.774872 & -0.029738 & 1 & -0.092964 & 3.765213 & -0.034646 \\
\hline 6 & 4.960969 & -0.085827 & 0.006817 & 6 & -4.882697 & 0.019338 & 0.006251 \\
\hline 8 & 5.242383 & 1.095037 & 0.004252 & 8 & -5.130856 & 1.207516 & 0.002628 \\
\hline 6 & 6.004771 & -1.191993 & 0.012198 & 6 & -5.954447 & -1.057711 & 0.012940 \\
\hline 1 & 5.896236 & -1.828085 & 0.897888 & 1 & -5.864479 & -1.701174 & -0.869307 \\
\hline
\end{tabular}




\begin{tabular}{|c|c|c|c|c|c|c|c|}
\hline 1 & 6.993864 & -0.734945 & 0.015204 & 1 & -6.932224 & -0.576782 & 0.014234 \\
\hline 1 & 5.902754 & -1.830231 & -0.872737 & 1 & -5.859237 & -1.695376 & 0.898834 \\
\hline 1 & -6.960659 & 1.243221 & 0.287448 & 1 & 7.067958 & 1.095888 & 0.298721 \\
\hline 7 & -6.659261 & 0.340296 & -0.051977 & 7 & 6.751538 & 0.202952 & -0.053805 \\
\hline 1 & -7.258829 & -0.410166 & 0.262239 & 1 & 7.333778 & -0.560424 & 0.262506 \\
\hline
\end{tabular}

Compound $7 \mathbf{i}$

\begin{tabular}{|c|c|c|c|c|c|c|c|}
\hline & & rotamer A & & & & rotamer B & \\
\hline Atomic number & $\mathbf{x}$ & $\mathbf{y}$ & $\mathbf{z}$ & Atomic number & $\mathbf{x}$ & $\mathbf{y}$ & $\mathbf{z}$ \\
\hline 6 & 1.996417 & -0.757312 & -0.004699 & 6 & -1.937672 & -0.740388 & -0.003449 \\
\hline 6 & 0.720108 & -0.066621 & -0.022134 & 6 & -0.653078 & -0.058279 & -0.022560 \\
\hline 6 & 3.166678 & 0.089098 & 0.002811 & 6 & -3.101748 & 0.116714 & 0.001909 \\
\hline 7 & 0.662198 & 1.275019 & -0.032756 & 7 & -0.574904 & 1.275631 & -0.036420 \\
\hline 7 & 3.090276 & 1.402862 & -0.008024 & 7 & -3.001624 & 1.434593 & -0.012571 \\
\hline 6 & 1.835088 & 1.923612 & -0.026926 & 6 & -1.746006 & 1.938861 & -0.032865 \\
\hline 7 & 4.387142 & -0.551762 & 0.022292 & 7 & -4.324795 & -0.503069 & 0.022941 \\
\hline 1 & 4.287913 & -1.564094 & 0.029016 & 1 & -4.227744 & -1.528188 & 0.031765 \\
\hline 7 & 2.224727 & -2.094612 & 0.006962 & 7 & -1.892547 & -2.096809 & 0.008508 \\
\hline 8 & 1.237804 & -2.887539 & 0.000680 & 8 & -2.967620 & -2.761650 & 0.026574 \\
\hline 7 & -0.392817 & -0.820733 & -0.027366 & 7 & 0.455104 & -0.824936 & -0.026033 \\
\hline 6 & -1.762685 & -0.483676 & -0.040460 & 6 & 1.832797 & -0.512645 & -0.040911 \\
\hline 1 & -0.156310 & -1.823651 & -0.020790 & 1 & 0.228586 & -1.816377 & -0.019314 \\
\hline 6 & -2.669021 & -1.556910 & -0.061358 & 6 & 2.724273 & -1.596084 & -0.077361 \\
\hline 6 & -4.041480 & -1.348540 & -0.068832 & 6 & 4.100453 & -1.405549 & -0.086204 \\
\hline 6 & -4.583104 & -0.040410 & -0.065612 & 6 & 4.659202 & -0.105792 & -0.069893 \\
\hline 6 & -2.283180 & 0.818750 & -0.021275 & 6 & 2.369623 & 0.781767 & -0.007100 \\
\hline 6 & -3.659959 & 1.029911 & -0.028769 & 6 & 3.749694 & 0.974643 & -0.016715 \\
\hline 7 & 1.788553 & 3.273213 & -0.040311 & 7 & -1.675585 & 3.286460 & -0.050584 \\
\hline 1 & -2.292042 & -2.576554 & -0.066345 & 1 & 2.336643 & -2.612179 & -0.093492 \\
\hline 1 & -4.689235 & -2.215794 & -0.078569 & 1 & 4.735984 & -2.281599 & -0.108143 \\
\hline 1 & -1.608801 & 1.661813 & 0.004431 & 1 & 1.706569 & 1.633339 & 0.030941 \\
\hline 1 & -4.011307 & 2.053846 & -0.006075 & 1 & 4.113461 & 1.993934 & 0.017107 \\
\hline 1 & 2.654215 & 3.789522 & -0.041659 & 1 & -2.532036 & 3.818176 & -0.054448 \\
\hline 1 & 0.900424 & 3.746882 & -0.062507 & 1 & -0.778964 & 3.743902 & -0.075440 \\
\hline 6 & 5.685596 & -0.023320 & 0.035304 & 6 & -5.614555 & 0.057065 & 0.033775 \\
\hline 8 & 5.945750 & 1.162544 & 0.027287 & 8 & -5.849574 & 1.248010 & 0.022352 \\
\hline 6 & 6.749745 & -1.109815 & 0.062433 & 6 & -6.698621 & -1.007410 & 0.063004 \\
\hline 1 & 6.649131 & -1.732197 & 0.958782 & 1 & -6.621366 & -1.666251 & -0.809017 \\
\hline 1 & 7.730202 & -0.634502 & 0.061819 & 1 & -7.670838 & -0.515321 & 0.062588 \\
\hline 1 & 6.663450 & -1.765377 & -0.811423 & 1 & -6.605223 & -1.631631 & 0.958647 \\
\hline 7 & -5.955633 & 0.179128 & -0.110449 & 7 & 6.036285 & 0.097211 & -0.119593 \\
\hline 6 & -6.859967 & -0.940488 & 0.103715 & 6 & 6.923644 & -1.036269 & 0.091677 \\
\hline 1 & -7.888514 & -0.590509 & 0.003674 & 1 & 6.755729 & -1.810693 & -0.664146 \\
\hline 1 & -6.704948 & -1.719211 & -0.650597 & 1 & 7.957300 & -0.701870 & -0.009936 \\
\hline 1 & -6.745038 & -1.398573 & 1.099601 & 1 & 6.803910 & -1.495310 & 1.086947 \\
\hline 6 & -6.467762 & 1.516413 & 0.146986 & 6 & 6.564054 & 1.421353 & 0.172037 \\
\hline 1 & -6.219673 & 1.881513 & 1.156974 & 1 & 6.176052 & 2.161594 & -0.535587 \\
\hline 1 & -6.074133 & 2.233307 & -0.581475 & 1 & 6.324107 & 1.762713 & 1.192553 \\
\hline 1 & -7.553731 & 1.507576 & 0.041868 & 1 & 7.649501 & 1.403385 & 0.061763 \\
\hline
\end{tabular}


Table S3. The calculated NH...O hydrogen-bond lengths in compounds 3a-7i ( $\AA$ ).

\begin{tabular}{ccc|ccc}
\hline Compound & $\begin{array}{c}\text { Rotamer A } \\
\mathrm{d}_{\mathrm{NH}} \ldots \mathrm{O}\end{array}$ & $\begin{array}{c}\text { Rotamer B } \\
\mathrm{d}_{\mathrm{NH}} \ldots \mathrm{O}\end{array}$ & Compound & $\begin{array}{c}\text { Rotamer A } \\
\mathrm{d}_{\mathrm{NH}} \ldots \mathrm{O}\end{array}$ & $\begin{array}{c}\text { Rotamer B } \\
\mathrm{d}_{\mathrm{NH}} \ldots \mathrm{O}\end{array}$ \\
\hline $\mathbf{3 a}$ & 1.718 & - & $\mathbf{6 a}$ & 1.743 & 1.901 \\
$\mathbf{3 b}$ & 1.721 & - & $\mathbf{6 b}$ & 1.747 & 1.898 \\
$\mathbf{3 c}$ & 1.725 & - & $\mathbf{6 c}$ & 1.751 & 1.894 \\
$\mathbf{3 d}$ & 1.721 & - & $\mathbf{6 d}$ & 1.747 & 1.892 \\
$\mathbf{3 e}$ & 1.728 & - & $\mathbf{6 e}$ & 1.753 & 1.887 \\
$\mathbf{3 f}$ & 1.728 & - & $\mathbf{6 f}$ & 1.754 & 1.886 \\
$\mathbf{3 g}$ & 1.722 & - & $\mathbf{6 g}$ & 1.749 & 1.885 \\
$\mathbf{3 h}$ & 1.722 & - & $\mathbf{6 h}$ & 1.749 & 1.881 \\
$\mathbf{3 i}$ & 1.723 & - & $\mathbf{6 i}$ & 1.750 & 1.880 \\
$\mathbf{4 a}$ & 1.734 & 1.842 & $\mathbf{7 a}$ & 1.748 & 1.786 \\
$\mathbf{4 b}$ & 1.738 & 1.839 & $\mathbf{7 b}$ & 1.752 & 1.783 \\
$\mathbf{4 c}$ & 1.743 & 1.836 & $\mathbf{7 c}$ & 1.756 & 1.780 \\
$\mathbf{4 d}$ & 1.738 & 1.834 & $\mathbf{7 d}$ & 1.752 & 1.777 \\
$\mathbf{4 e}$ & 1.745 & 1.829 & $\mathbf{7 e}$ & 1.759 & 1.773 \\
$\mathbf{4 f}$ & 1.746 & 1.828 & $\mathbf{7 f}$ & 1.759 & 1.771 \\
$\mathbf{4 g}$ & 1.740 & 1.827 & $\mathbf{7 g}$ & 1.753 & 1.771 \\
$\mathbf{4 h}$ & 1.740 & 1.823 & $\mathbf{7 h}$ & 1.753 & 1.766 \\
$\mathbf{5 a}$ & 1.734 & 1.842 & $\mathbf{7 i}$ & 1.754 & 1.763 \\
$\mathbf{5 b}$ & 1.737 & 1.838 & & & \\
$\mathbf{5 c}$ & 1.742 & 1.836 & & & \\
$\mathbf{5 d}$ & 1.737 & 1.834 & & & \\
$\mathbf{5 e}$ & 1.745 & 1.829 & & & \\
$\mathbf{5 f}$ & 1.745 & 1.827 & & & \\
$\mathbf{5 g}$ & 1.739 & 1.827 & & & \\
$\mathbf{5 h}$ & 1.740 & 1.823 & & & \\
\hline
\end{tabular}

